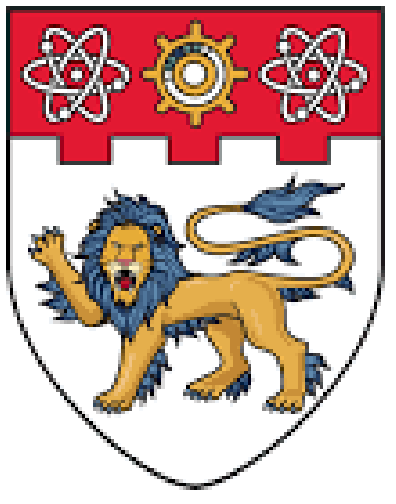

NANYANG TECHNOLOGICAL UNIVERSITY SINGAPORE

\author{
PEPTIDE-BASED ANTIBACTERIAL
} AGENTS TO OVERCOME ANTIBIOTIC RESISTANCE

\author{
AHMAD HUSSEN TAREQ
}

SCHOOL OF BIOLOGICAL SCIENCES

$\stackrel{ }{\vartheta}$ 


\section{PEPTIDE-BASED ANTIBACTERIAL AGENTS TO OVERCOME ANTIBIOTIC RESISTANCE}

\section{AHMAD HUSSEN TAREQ}

\section{SCHOOL OF BIOLOGICAL SCIENCES}

A thesis submitted to the Nanyang Technological University in partial fulfillment of the requirement for the degree of Doctor of Philosophy 


\section{ACKNOWLEDGEMENT}

It has been an emotional journey with countless exciting and several testing moments. I have learned a lot, found new friends and in all that I discovered myself. My deepest gratitude goes to my mentors, colleagues and friends for their continuous support and contribution throughout the journey.

Firstly, I would like to express my sincere gratitude to my advisor Prof. Dr. Liu Chuan-Fa for the continuous support of my $\mathrm{PhD}$ study and related research, for his patience, encouragement, and immense knowledge. His guidance helped me in all the time of research and the writing of this thesis. I could not have imagined having a better advisor and mentor for my PhD study.

Besides my advisor, I would like to thank Prof. James P. Tam, whose lab also provided the needed support. Without their precious support it would not be possible to conduct this research.

I thank my fellow lab mates for the stimulating discussions, for the sleepless nights we were working together before deadlines, and for all the fun we have had in the last four years. I started to learn peptide synthesis from Dr Zhang Xiaohong, then learned a lot from Dr Yang Renliang. Having great buddies like Dr Bi Xiao Bao and Dr Cao Yuan, we shared similar journeys of rigors of $\mathrm{PhD}$ training together. I am also thankful to Ding Yingjie for her supporting attitude and willingness to help, which really assisted me a lot and to Dr Biplab Banerjee for all the technical discussions and help I got from him. Rao Chang helped me in a lot of work, for which I am really grateful to her. She helped me tremendously in my projects from discussions to conducting experiments of peptide synthesis. One of the best mentors and a friend, Dr Kalyan Kumar Pasunooti, who taught me many things, pushed me when I needed the push, and always helped me to improve. I am thankful to my buddies and housemates for the memorable time we spent together. 
I am blessed to have friends like Areetha Dsouza for helping me perform NMR experiments at a critical moment even though she was quite busy in her work, and for her patience, guidance and kindness. I value all the discussions I had with her on NMR and other biophysical analyses. I am really thankful to Anjali Sengar for all her help and support. I am thankful to Manikantha for helping me doing the HRMS. I am thankful to all my friends in the lab and in NTU for making these past years so memorable. I am grateful for all the opportunities and support I got from NTU for my personal and professional development.

Last but not the least, I would like to thank my family: my parents and my brothers, sisters and sister in law for supporting me spiritually throughout writing this thesis and my life in general. I can never thank them enough. I am blessed to have a wonderful family, who are always there for me, especially in hard times. It is ineffable to describe their support, love and care for me. 


\section{TABLE OF CONTENTS}

ACKNOWLEDGEMENT ...............................................................................i

TABLE OF CONTENTS ................................................................... iii

LIST OF FIGURES ..................................................................................... vii

LIST OF TABLES .............................................................................. xiii

ABBREVIATIONS ........................................................................................xiv

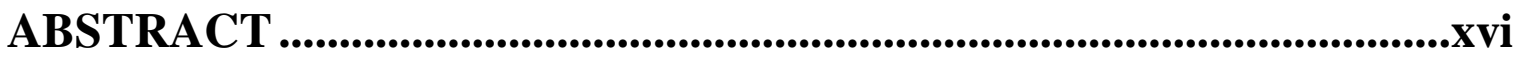

CHAPTER 1 GENERAL INTRODUCTION ..............................................1

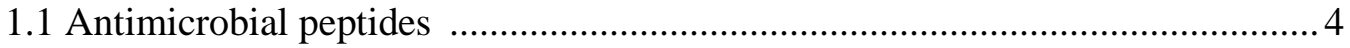

1.2 Modification of existing antibiotics ................................................................ 5

1.3 Accessing the untapped resources ..............................................................

1.4 Aims and significance of the study ............................................................... 8

\section{CHAPTER 2 PREPARING DIMERS OF MEMBRANE-ACTIVE}

ANTIMICROBIAL PEPTIDES ...............................................................11

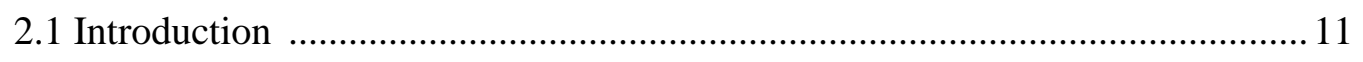

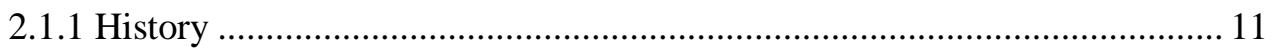

2.1.2 Importance of antimicrobial peptides ................................................... 11

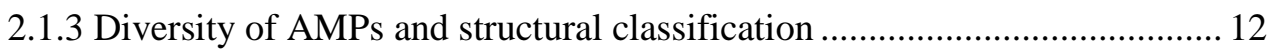

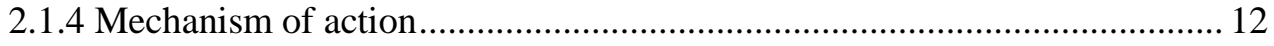

2.1.5 Design of synthetic AMPs ……………………………………………. 14

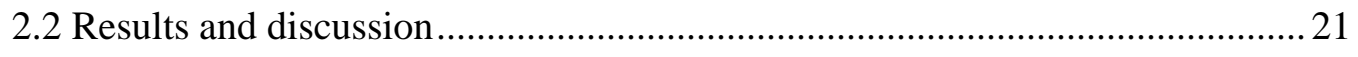

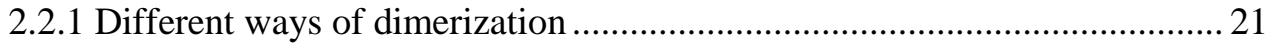

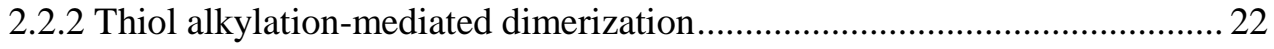

2.2.3 HBD-3-derived V2 peptides and their dimerized versions ........................... 23

2.2.4 The bivalent linker for $\mathrm{V} 2$ peptide dimerization.................................... 27

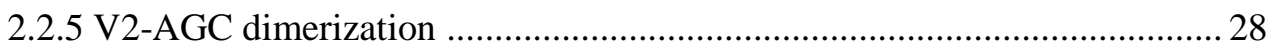

2.2.6 V2-Cys dimerization............................................................................... 30 


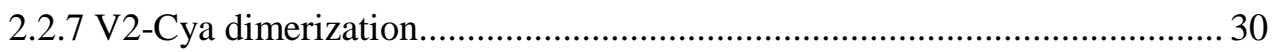

2.2.8 Antibacterial activities of V2 peptide derivatives ..................................... 32

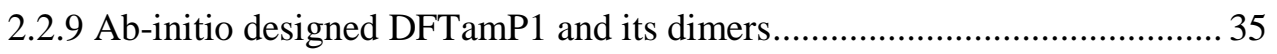

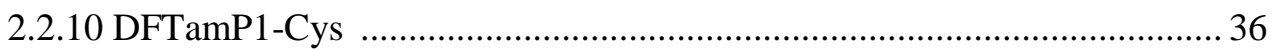

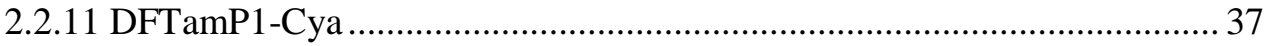

2.2.12 Dimerization of DFTamP1-Cys with di-bromoacetyl linker ..................... 37

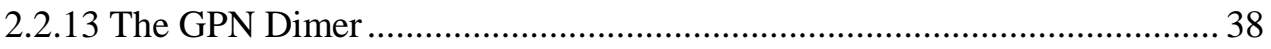

2.2.14 The parallel DFTamP1-p dimer ............................................................ 39

2.2.15 Radial diffusion assays of DFTamP1-p and its variants ......................... 40

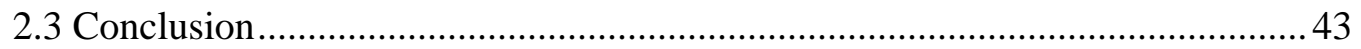

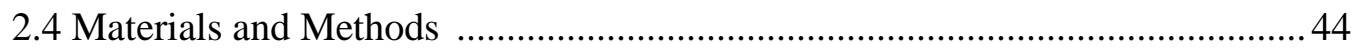

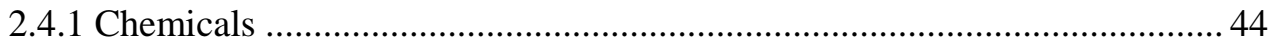

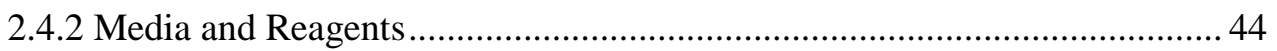

2.2.3 Solid Phase Peptide Synthesis .............................................................. 45

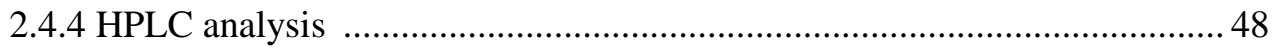

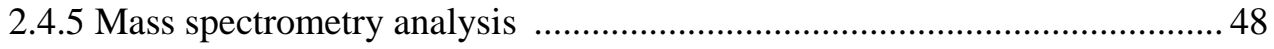

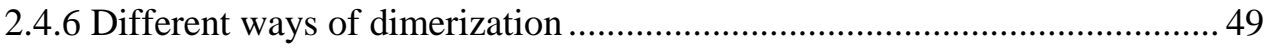

2.4.6.1 Preparation of $\alpha, \varepsilon$-dibromoacetyl-Lys-Lys- $\mathrm{NH}_{2} \ldots \ldots \ldots \ldots \ldots \ldots \ldots \ldots \ldots . . . . \ldots \ldots$

2.4.6.2 Dimerisation of V2-Cys on the bivalent core $\alpha, \varepsilon$-dibromoacetyl-

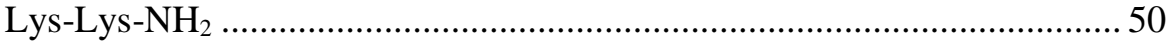

2.4.6.3 Dimerisation of V2-Cya with the di-bromoacetyl linker................ 50

2.4.6.4 Dimerisation of Cys-attached DFTamP1-p with di-bromoacetyl

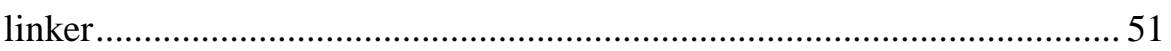

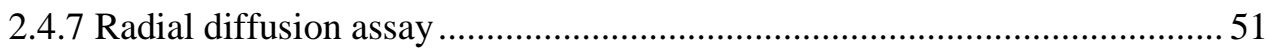

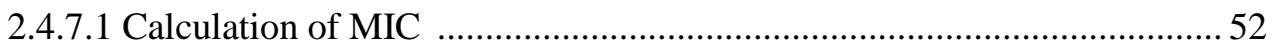

\section{CHAPTER 3 VANCOMYCIN C-TERMINAL AMIDE ANALOGS ....53}

3.1 Introduction of glycopeptides......................................................................53

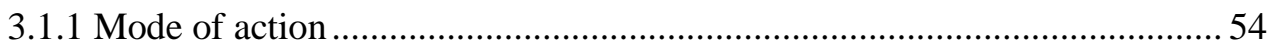




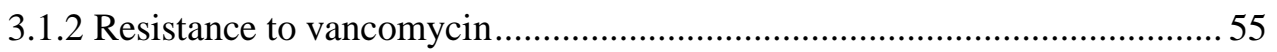

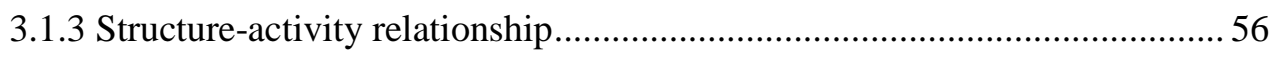

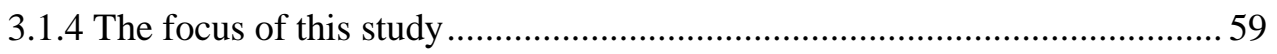

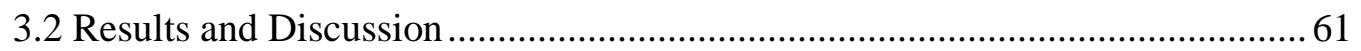

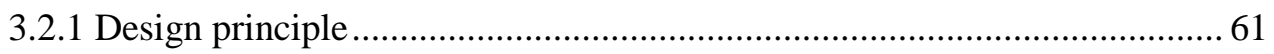

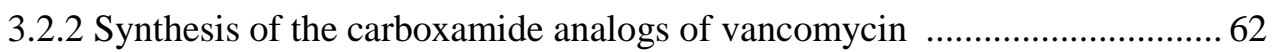

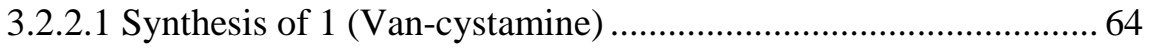

3.2.2.3 Synthesis of 3 (Van-ethylenediamine) ....................................... 66

3.2.2.4 Synthesis of 4 (Van-dimethylethylenediamine) .......................... 67

3.2.2.5 Synthesis of 5 (Van-trimethylethylenetriamine) ......................... 68

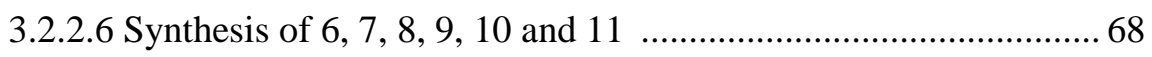

3.2.2.7 Synthesis of 12 (Van-O-phenylenediamine) ............................. 72

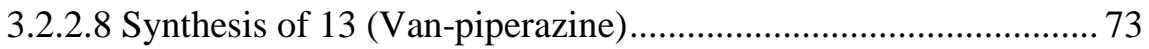

3.2.2.9 Synthesis of 14 (Van-diethylenetriamine) ................................ 73

3.2.3 Antimicrobial studies of different analogs ............................................ 74

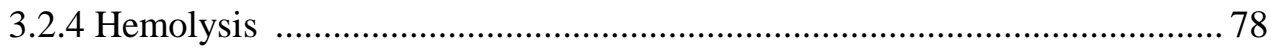

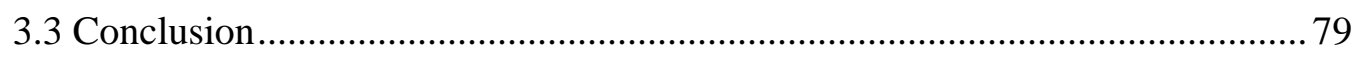

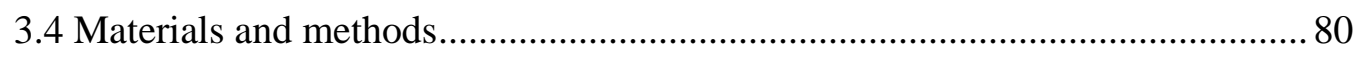

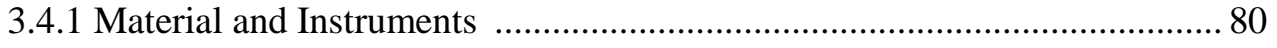

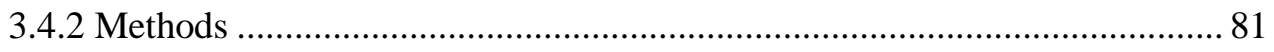

\section{CHAPTER 4 SYNTHESIS OF TEIXOBACTIN ANALOGS.................84}

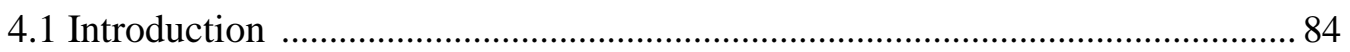

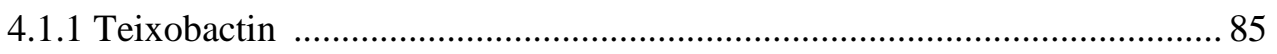

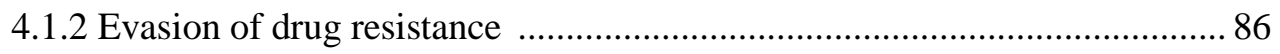

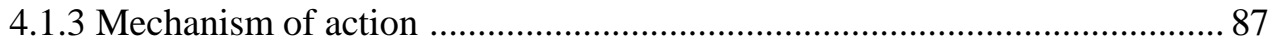

4.1.4 Reported syntheses of teixobactin analogs ............................................ 88

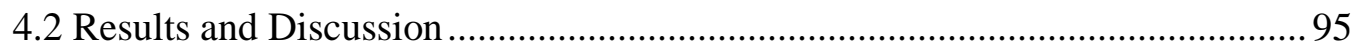

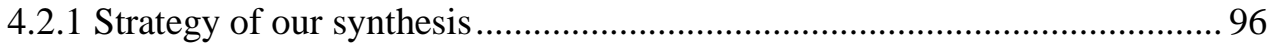


4.2.2 Loading Fmoc-Cys-OH onto trityl resin

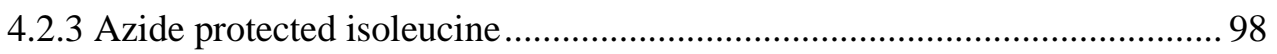

4.2.4 Optimization of the on-rein esterification reaction ................................... 98

4.2.5 Two routes for the synthesis of teixobactin analogs ............................... 101

4.2.6 Synthesis of the Arg10-teixobactin analogs by synthetic route A ............. 102

4.2.6.1 The choice of protecting group for the $\mathrm{C}$-ter $\mathrm{COOH}$................. 103

4.2.6.2 Macrolactamization ............................................................. 103

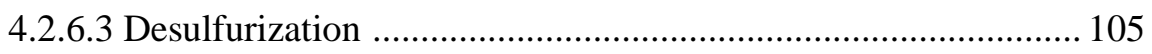

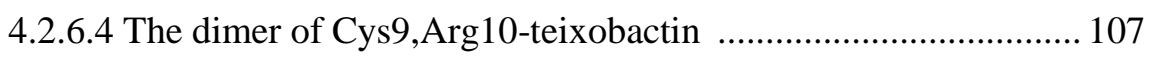

4.2.7 The synthesis of other analogs with Lys, Orn or His at position 10 .......... 108

4.2.7.1 Formation of the cyclic depsipeptide ring in the synthesis of the

Lys, Orn or His10 analogs of teixobactin

4.2.7.2 Completing the synthesis of Cys9,Lys10-teixobactin,

Cys9,Orn10-teixobactin and Cys9,His10-teixobactin

4.2.7.3 Desulfurization of Cys9,Lys10-teixobactin,

Cys9,Orn10-teixobactin, and Cys9,His10-teixobactin

4.2.7.4 The dimers of Cys9,Lys10-teixobactin, Cys9, Orn10-teixobactin and Cys9,His10-teixobactin ..................................................... 116

4.2.8 Synthesis of teixobactin analogs via route $\mathrm{B}$....................................... 118

4.2. 9 Antimicrobial activities of teixobactin analogs .................................. 120

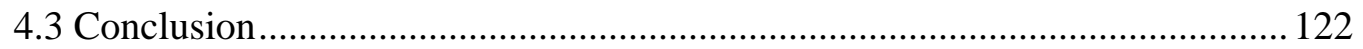

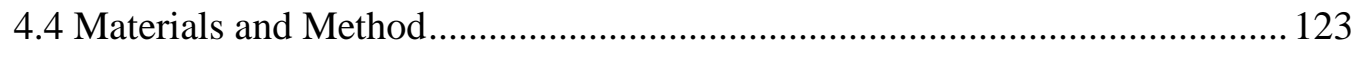

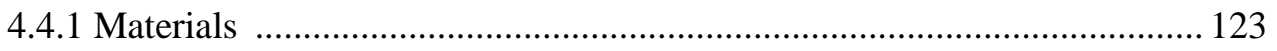

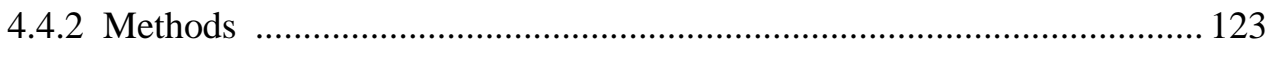

CHAPTER 5 GENERAL CONCLUSION .........................................129

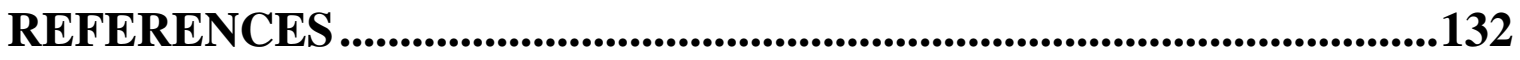

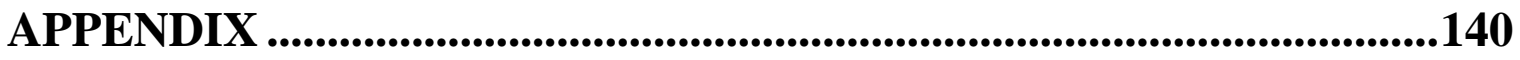




\section{LIST OF FIGURES}

\section{CHAPTER 1}

Figure 1.1 The discovery of antibiotics in different decades of $20^{\text {th }}$ century.............................. 1

Figure 1.2 Timeline for the emergence of antibiotic resistance ..............................................

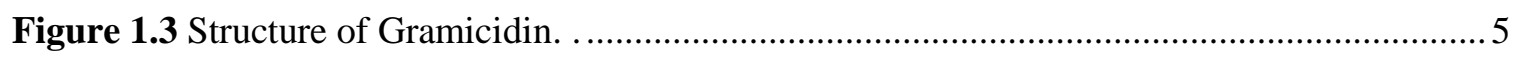

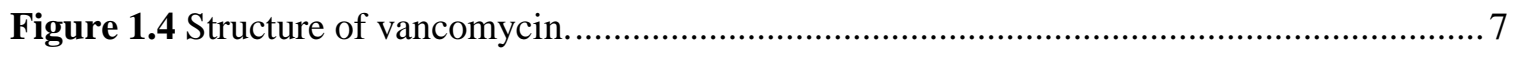

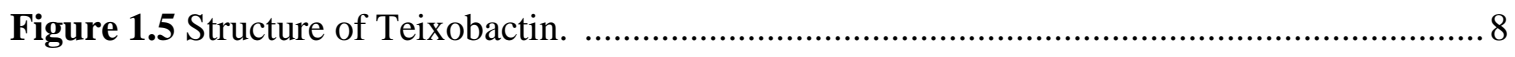

\section{CHAPTER 2}

Figure 2.1 The listed models: (A) carpet, (B) barrel-stave, (C) wormhole or toroidal, and (D)

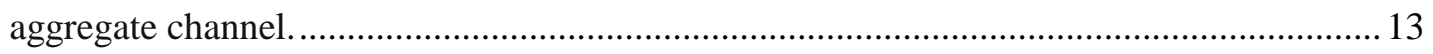

Figure 2.2 Ribbon model representation of DFTamP1-p (GLLPLLSLLGKLL). ..................... 15

Figure 2.3 Three types of dendrimeric cores with three generations of lysines, A. Two branched Lys; B. Four branched (Lys) $)_{2}$ Lys: C. eight-branched $\left[(\text { Lys })_{2} \text { Lys }\right]_{2}$ Lys. ............................. 16

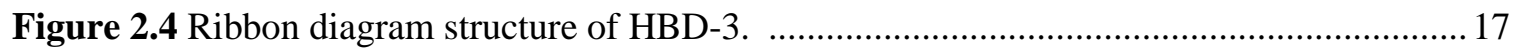

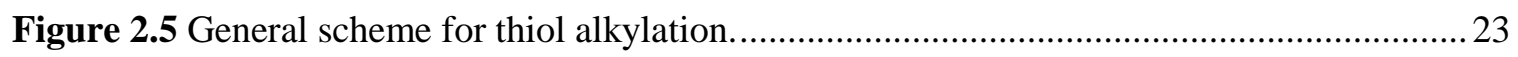

Figure 2.6 C18 analytical HPLC profile of the pure V2 dimer. .............................................24

Figure 2.7 C18 analytical HPLC profile of pure V2-AGC. .................................................26

Figure 2.8 The di-bromoacetyl-KK-NH2 linker used in thiol alkylation. ..................................28 
Figure 2.9 Reaction of V2-AGC with the di-bromoacetyl linker to form the V2-AGC dimer.....29

Figure 2.10 HPLC monitoring of V2-AGC dimerization reaction. .29

Figure 2.11 C18 analytical HPLC profile of purified V2-AGC dimer. .29

Figure 2.12 Left panel: the structure of V2-Cys. Right panel: V2-Cys dimer. .30

Figure 2.13 C18 analytical HPLC profile of pure V2-Cys dimer. .30

Figure 2.14 Structures of V2-Cya and V2-Cys dimer. .31

Figure 2.15 C18 analytical HPLC profile of purified V2-Cya dimer. 31

Figure 2.16 C18 analytical HPLC profile of DFTamP1-p. .35

Figure 2.17 C18 analytical HPLC profile of pure DFTamP1-Cys 36

Figure 2.18 Structure of DFTamP1-Cya. .37

Figure 2.19 C18 analytical HPLC profile of pure DFTamP1-Cya. .37

Figure 2.20 Structure of DFTamP1-Cys dimer formed by the thiol-alkylation reaction. .38

Figure 2.21 C18 analytical HPLC profile of pure DFTamP1-Cys dimer. .38

Figure 2.22 GPN linked dimer. .39

Figure 2.23 C18 analytical HPLC profile of purified GPN dimer. .39

Figure 2.24 Structure of the parallel DFTamP1-dimer. 40

Figure 2.25 C18 analytical HPLC profile of DFTamP1-dimer. .40

Figure 2.26 Conjugation/dimerization of peptides using the thiol alkylation reaction. 49 


\section{CHAPTER 3}

Figure 3.1 The structure of vancomycin.

Figure 3.2 The complex between the precursor peptidoglycan terminal peptide N-acyl-L-Lys-D-

Ala-D-Ala-OH and vancomycin binding pocket through five hydrogen bonds. .54

Figure 3.3 Hydrogen bond interactions between vancomycin and the C-ter dipeptide of the peptidoglycan.

Figure 3.4 Functional sites on vancomycin for structural moficiation to synthesize analogs.......56

Figure 3.5 Some modification sites in vancomycin.

Figure 3.6 The general scheme of reaction between the carboxyl group of vancomycin and an amine.

Figure 3.7 The structure of Van-cystamine 1

Figure 3.8 Analytical HPLC profile and the ESI-MS spectrum of the purified Van-cystamine analog 1.

Figure 3.9 Structure of Van-cysteamine. Analytical HPLC profile and the ESI-MS of the purified Van-cysteamine analog 2.

Figure 3.10 Structure of Van-ethylenediamine. Analytical HPLC profile and the ESI-MS of the purified Van-ethylenediamine 3.

Figure 3.11 Analytical HPLC profile and the ESI-MS of the purified N, N, dimethyl ethylene diamine analog 4 .

Figure 3.12 Analytical HPLC profile and the ESI-MS of the purified N, N, N-trimethyl-ethylenetriamine analog $\mathbf{5}$.

Figure 3.13 Analytical HPLC profile and the ESI-MS of Van-dimethylamino-propylamine 6. .69 
Figure 3.14 Analytical HPLC profile and the ESI-MS of Van-diaminopropane 7

Figure 3.15 Analytical HPLC profile and the ESI-MS of Van-diaminobutane 8. .70

Figure 3.16 Analytical HPLC profile and the ESI-MS Van-diaminopentane 9. .70

Figure 3.17 Analytical HPLC profile and the ESI-MS of Van-diaminohexane 10. .71

Figure 3.18 Analytical HPLC profile and the ESI-MS of Van-diaminoheptane 11. .71

Figure 3.19 Analytical HPLC profile and the ESI-MS of Van-O-phenylene-diamine 12. .72

Figure 3.20 Analytical HPLC profile and the ESI-MS of Van-piperazine 13. .73

Figure 3.21 Analytical HPLC profile and the ESI-MS of the Van-diethylenetriamine 14. 74

\section{CHAPTER 4}

Figure 4.1 The scheme to synthesize teixobactin analog using solid phase peptide strategy employed by Jad et al.

Figure 4.2. Solid phase employed by Parmer et al. to synthesize teixobactin analog.

Figure 4.3 Scheme used by Yang et al, to synthesize different teixobactin analogs.

Figure 4.4 Synthesis of teixobactin analog using strategy employed by Giltrap et al. .93

Figure 4.5 Our general strategy for the synthesis of teixobactin and its analogs. .96

Figure 4.6 Cysteine linked resin and trityl protection a) and b).

Figure 4.7 Preparation of azido-isoleucine. .98

Figure 4.8 A representative esterification reaction scheme on solid phase. .99

Figure 4.9 Analytical HPLC profile and ESI-MS of the crude ester product of peptide 5....... 100 
Figure 4.10 Two routes to synthesize teixibactins using the on-resin cyclization approach. $\mathrm{R}=$ trityl or 2-Cl-trityl.

Figure 4.11 Synthesis of the Arg10-analog of teixobactin via Route A. 102

Figure 4.12 HPLC and MS monitoring of the cyclization reaction on solid phase. Peptide 8 before cyclization and the cyclized peptide 9 . 104

Figure 4.13 Analytical HPLC profile and the ESI-MS of pure Cys9,Arg10-teixobactin 11.... 105

Figure 4.14 Desulfurization of Cys9,Arg10-teixobactin 11 to afford Arg10-teixobactin. 106

Figure 4.15 Analytical HPLC of the desulfurization reaction mixture using 20\% AcOH solution and ESI-MS of the isolated desulfurization product 11a. 106

Figure 4.16 Structure of the dimer of Arg-teixobactin formed through a disulphide bond. 108

Figure 4.17 Analytical HPLC profile of the S-S Arg-teixobactin dimer 11b and its ESI-MS spectrum. 108

Figure 4.18 Structure of the hexapeptides with the C-ter cyclodepsitetrapeptide ring. The thick red bond was formed at the on-resin cyclization step. 109

Figure 4.19 HPLC profile of the linear hexapeptide with Lys10 pre-cyclization and the cyclic product post-cyclization after microcleavage.

Figure 4.20 HPLC profile of the linear hexapeptide with Orn10 pre-cyclization and the cyclic product post-cyclization after microcleavage. 110

Figure 4.21 HPLC profile of the linear hexapeptide with His10 pre-cyclization and the cyclic product post-cyclization after microcleavage. 110

Figure 4.22 Lys10 analog of teixobactin 12. 111

Figure 4.23 Analytical HPLC profile and ESI-MS mass spectrum of Cys9,Lys10-teixobactin. 112 
Figure 4.24 Structure of Cys9, Orn10-Teixobactin 13. 112

Figure 4.25 Analytical HPLC of Cys9, Orn10-teixobactin 13 and its ESI-MS mass spectrum. .113

Figure 4.26 Stucture of Cys9, His10-Teixobactin 14 . 113

Figure 4.27 Analytical HPLC and ESI-MS mass spectrum of Cys9,His10-teixobactin. 114

Figure 4.28 Analytical HPLC and ESI-MS spectrum of Lys10-teixobactin 12a. 114

Figure 4.29 Analytical HPLC of Orn10-teixobactin 13a and its ESI-MS mass spectrum 115

Figure 4.30 Analytical HPLC and ESI-MS of His10-teixobactin 14a. 116

Figure 4.31 Structures of S-S dimers $12 \mathrm{~b}, \mathbf{1 3 b}$ and $\mathbf{1 4 b}$. 116

Figure 4.32 Analytical HPLC of 12b its MALDI-TOF MS. 117

Figure 4.33 Analytical HPLC and MALDI-TOF MS of Cys9, Orn10-teixobactin dimer 13b. .118

Figure 4.34 Analytical HPLC and MALDI-TOF MS of Cys9,His10-teixobactin dimer 14b. . 118

Figure 4.35 $\beta$-Elimination of the Thr ester during piperidine exposure. 119

Figure 4.36 Analytical HPLC profile of NMe-D-Phe-Ile-Ser-D-Gln-D-allo-Ile-Ser-D-Thr(ON3Ile)-Cys-OH after microcleavage and ESI-MS. 119 


\section{LIST OF TABLES}

\section{CHAPTER 2}

Table 2.1 List of all V2-related peptides synthesized in this study......................................... 25

Table 2.2 MIC of V2 monomer and V2-AGC peptide............................................................. 27

Table 2.3 MIC $(\mu \mathrm{M})$ of V2 peptides and the dimers against different bacterial strains................. 32

Table 2.4 List of all DFTamP1-p related peptides synthesized in this study.............................. 36

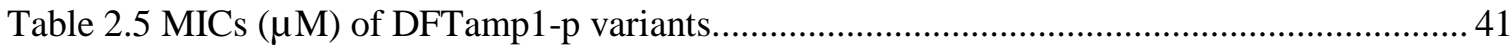

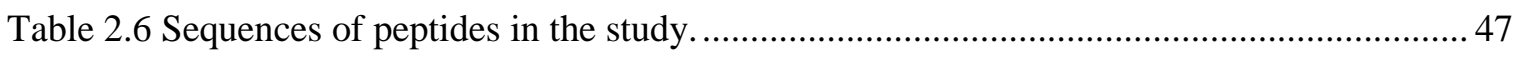

\section{CHAPTER 3}

Table 3.1 The antibacterial activities of different carboxamide analogs of vancomycin.............. 75

Table 3.2 The hemolytic activity of different vancomycin analogs. .......................................... 79

\section{CHAPTER 4}

Table 4.1 Optimization of on-resin esterification oconditions with different peptides............... 100

Table 4.2 Antimicrobial activity of different teixobactin analogs........................................... 121 


\section{ABBREVIATIONS}

\begin{tabular}{|c|c|}
\hline $\mathrm{ACN}$ & Acetonitrile \\
\hline B. subtilis & Bacillus subtilis \\
\hline $\mathrm{CHCA}$ & alpha-Cyano-4-hydroxycinnamic acid \\
\hline $\mathrm{ddH}_{2} \mathrm{O}$ & Deionized Distilled Water \\
\hline DIEA & N,N-Diisopropylethylamine \\
\hline DMF & Dimethylformamide \\
\hline E. coli & Escherichia coli \\
\hline ESI & Electrospray Ionization \\
\hline $\mathrm{HCL}$ & Hydrochloric acid \\
\hline hVISA & $\begin{array}{l}\text { Heteroresistant Vancomycin-intermediate Staphylococcus } \\
\text { aureus }\end{array}$ \\
\hline MALDI & Matrix-assisted Laser Desorption/Ionization \\
\hline MIC & Minimum Inhibitory Concentration \\
\hline MRSA & Methicillin-resistant Staphylococcus aureus \\
\hline NAG & $\mathrm{N}$-acetylglucosamine \\
\hline NAM & $\mathrm{N}$-acetylmuramic acid \\
\hline P. aeruginosa & Pseudomonas aeruginosa \\
\hline RP-HPLC & Reversed-phase High-performance Liquid Chromatography \\
\hline S. aureus & Staphylococcus aureus \\
\hline
\end{tabular}


TSB

VISA

VRE

VRSA

CTC

DIC

DIEA

DCM

DMAP

DMF

EDT

ESI

HATU

PyBOP

MIC

TCEP

TFA

TSB

TIS

SPPS
Tryptic Soy Broth

Vancomycin-intermediate Staphylococcus aureus

Vancomycin-resistant enterococci

Vancomycin-resistant Staphylococcus aureus

Chlorotrityl Chloride

Diisopropylcarbodiimide

N,N-Diisopropylethylamine

Dichloromethane

4-(Dimethylamino)pyridine

Dimethylformamide

1,2-Ethanedithiol

Electrospray Ionization

1-[Bis(dimethylamino)methylene]-1H-1,2,3-triazolo[4,5-

b]pyridinium 3-oxid hexafluorophosphate

((1H-Benzo[d][1,2,3]triazol-1-yl)oxy)tri(pyrrolidin-1yl)phosphonium hexafluorophosphate(V)

Minimum Inhibitory Concentration

Tris (2-Carboxyethyl) phosphine Hydrochloride

Trifluoroacetic acid

Trypticase soy broth

Triisopropylsilane

Solid phase peptide synthesis 


\section{ABSTRACT}

Conventional antibiotics are becoming increasingly ineffective as a result of bacterial resistance, and multidrug-resistant bacteria are a severe threat to public health. Hence, it is imperative to find new antibacterial strategies to address this serious problem. Targeting the bacterial membrane or cell wall offers an attractive strategy. Bacteria cannot simply change its membrane or essential cell-wall components; therefore, anti-bacterial agents targeting those sites offer effective solutions to the problem of drug resistance. Based on this rationale, three different strategies are pursued and developed in this thesis.

Membrane-active antimicrobial peptides (AMPs) are attractive antibacterial candidates. Dimerization of existing AMPs often can increase their potency and stability. We developed a dimer formation strategy by ligating two peptides together through thiol alkylation. This method addresses some limitations of solid phase synthesis methods in preparing long peptide dimers. We observed that dimerization improves efficacy of a highly cationic peptide (i.e., HBD-3) but diminishes the activity of a hydrophobic peptide called DFTamP1-p. It suggests that different mechanisms of action are involved for the cationic peptide and the hydrophobic peptide in bacterial killing.

Glycopeptide antibiotics interrupt bacterial cell wall synthesis to exert their antibacterial effects, and as such command a high barrier for drug resistance development. Modification of glycopeptide antibiotics to enhance their activity is therefore a useful strategy to develop new compounds against drug-resistant strains. We explored an underutilized reactive site on the glycopeptide antibiotics and developed a simple yet highly efficient scheme to synthesize various analogs. Using this scheme, the C-terminal carboxyl group of vancomycin was reacted with amine compounds to yield carboxamide analogs some of which with improved antibacterial activity. Usually multiple chemical reactions are needed to prepare antibiotic analogs. Our single-step scheme provides a simple yet efficient methodology to develop potent analogs of vancomycin. 
The recently discovered teixobactin is a novel and highly potent cyclodepsipeptide which targets an essential cell wall component lipid II and thus can evade bacterial drug resistance. We developed a new method to synthesize teixobactin-like cyclodepsidepeptides. In our robust method, we used solid-phase esterification and lactonization to form the cyclic depsi-tetrapeptide core, followed by extension of the Nterminal tail to form the full-length teixobactin molecule. Using this scheme, we synthesized various analogs of teixobactin and their respective dimers. Antimicrobial studies were conducted and some analogs showed activity in the therapeutic range. 


\section{CHAPTER 1 GENERAL INTRODUCTION}

The introduction of antibiotics is recognized as one of the greatest medical achievements in mankind history. Beginning with the discovery of penicillin in the late 1920 s, the antibiotic age saw its golden era in the 1940s to 1960s when the work of microbiologist Selman Waksman and others led to the isolation of almost all main classes of antibiotics known today (Figure 1.1). This success was attributed to the use of the socalled Waksman platform of screening cultured soil microbes for active secondary metabolites which included streptomycin, an aminoglycoside and the first drug effective against tuberculosis, and other important antibiotics. However, this platform started to show its limitations after the 1960s as the resource was getting exhausted and fewer and fewer new antibacterial compounds have been uncovered since (Figure 1.1). ${ }^{1}$ It is evident that researchers need to take other alternative avenues to access untapped resources in order to isolate completely new antibiotics from the natural world.

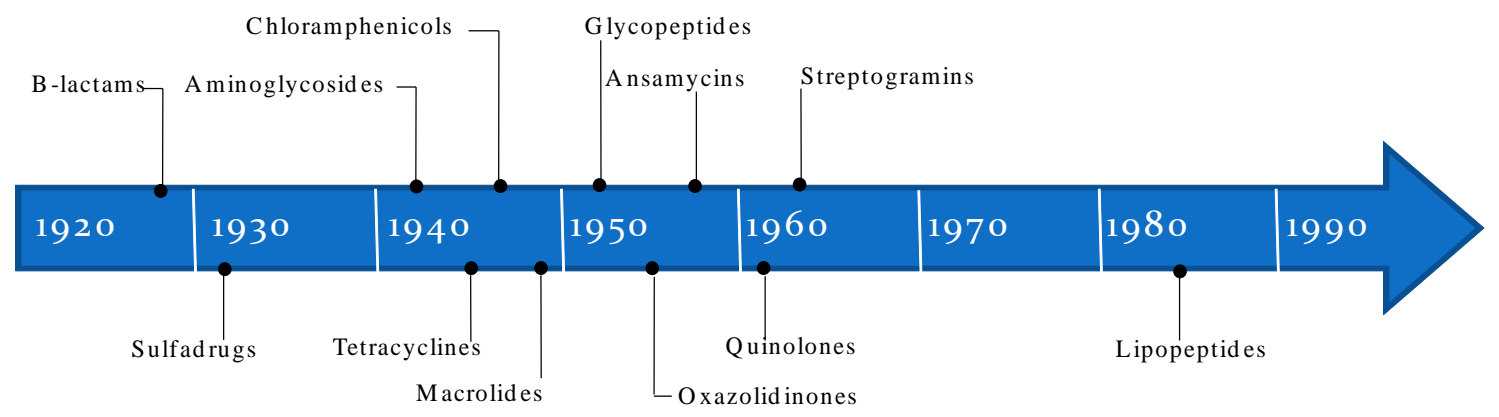

Figure 1.1 The discovery of antibiotics in different decades of $20^{\text {th }}$ century. ${ }^{1}$

Accompanying the use of antibiotics has been the emergence of drug-resistant bacterial strains (Figure 1.2). Soon after penicillin was introduced into clinics, resistant strains of Staphylococcus aureus were reported. ${ }^{2}$ To resolve this problem, penicillin synthetic analogs, e.g., methicillin, were developed which were less susceptible to $\beta$ lactamases, the enzymes responsible for the deactivation of $\beta$-lactam antibiotics. However, 
after its introduction, methicillin-resistant Staphylococcus aureus (MRSA) strains soon began to appear. ${ }^{3}$ In addition to discovering natural antibiotics, medicinal chemists have also directed their efforts to design completely synthetic antimicrobials. A breakthrough came in 1962 when nalidixic acid, the first synthetic antibiotic inhibiting bacterial DNA gyrase, was developed. Nevertheless, the rapid spread of drug-resistant microbes constitutes to be a serious challenge to health care system worldwide. ${ }^{4}$ The global crisis of drug-resistant bacteria is becoming more complicated as bacteria are developing resistance to multiple antibacterial agents. Such strains are becoming more prevalent, and mortality rates due to multi-drug resistant bacterial infection are high. For instance, 63000 patients die in the USA due to drug-resistant infections every year. ${ }^{5}$ Due to a huge deficit in the discovery of new antibiotics, we are losing the battle against resistant strains as some bacterial infections which were under control for decades are now resistant to most antibiotics. Some of those drug-resistant strains include MRSA, VRE (vancomycin resistant enterococci), VRSA (vancomycin resistant Staphylococcus aureus), and XDRTB (extremely drug resistant tuberculosis). Notably, VRE, which is evolved from Enterococci - part of natural ecological flora in the human gut, causes fatal endocarditis and is the second most common cause of hospital-acquired infections. ${ }^{6} 90 \%$ of the VRE strains are now ampicillin-resistant. The mobility of resistant genes from VRE also caused various other Gram-positive bacteria such as Staphylococcus aureus to become vancomycin-resistant, and the resultant VRSA is responsible for increased morbidity and mortality. ${ }^{7}$

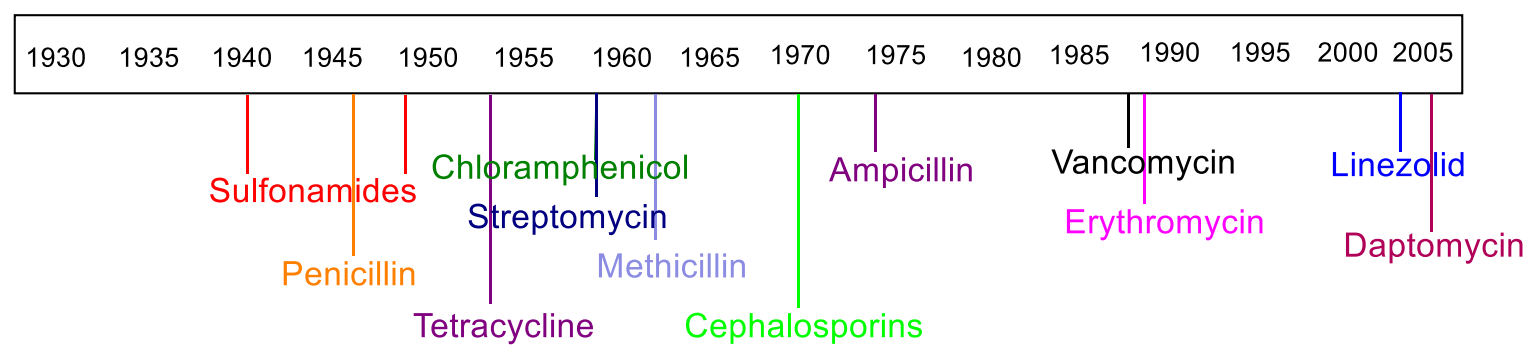

Figure 1.2 Timeline for the emergence of antibiotic resistance. ${ }^{8}$ 
A particularly dangerous situation is seen with Mycobacterium tuberculosis which has developed resistance to many known antibiotics used for its therapy. ${ }^{9-10}$ One-third of the world's population are infected with Mtb, though many in the latent or dormant stage. In 2014, 9.6 million additional TB cases were reported and the significant proportion of them with a drug-resistant strain. ${ }^{11}$ This archaic pathogen caused more human deaths than any other diseases. In current therapeutic regimens, multiple antibiotics are prescribed to treat TB. Still, the bacteria manage to consistently compromise the anti-TB treatment. ${ }^{12}$ There are reports of extremely drug-resistant Mtb strains not sensitive to most clinically available antimicrobials. ${ }^{13}$ There are certain strains reported to be resistant to all first and second line drugs used for Mtb therapy, and some published reports refer them as Totally Drug Resistant (TDR)-TB. ${ }^{14}$ So for diseases like TB, we have almost reached a point where no effective treatment is available for patients infected with TDR-TB.

Bacteria employ a complex set of mechanisms to develop resistance, and they can spread the resistant genes among different strains, even different species. ${ }^{15}$ As it is incessantly challenging to manage drug-resistant infection with existing drugs, researchers have been exploring new avenues for drug discovery to overcome this problem. The first strategy resides with the use of antimicrobial peptides or AMPs which target bacterial components that are difficult to change or evolve. Secondly, improvement of currently used antimicrobials by synthetic analogs continues to be an effective strategy to develop new antibiotics that are effective against resistant strains. Thirdly, the recent advances in genomic and metabolomic approaches can augment traditional methods of studying natural products. ${ }^{16}$ The untapped sources from nature can always provide us with an abundant variety of diverse compounds. An improved Waksman platform with different culturing techniques can certainly help to gain access to these previously inaccessible compounds. 


\subsection{Antimicrobial peptides}

Naturally existing antimicrobial peptides (AMPs) are a component of the innate immune system in nearly all organisms from prokaryotes to humans. Many AMPs target bacterial cytoplasmic membrane to exert their antibacterial effects. As such, it is difficult for bacteria to develop resistance against those AMPs. Interestingly, most of the AMPs are effective against drug-resistant bacteria. They are quite selective toward their targets with less toxicities and have the ability to synergize with conventional antibiotics. ${ }^{17}$ Some AMPs were found to be active as broad-spectrum antimicrobials, or as anti-endotoxin agents. ${ }^{18}$ The first AMPs were discovered in 1939. With the odds of discoverying natural antibiotics declining dramatically after the 1970s, AMPs garnered the interest of researchers. Significant research has been conducted to isolate, investigate and synthesize various AMPs. More than 2000 AMPs have been discovered and isolated from various organisms. ${ }^{17}$ Natural and synthetic AMPs have strong potential as antibacterials against resistant microbes and are considered as next-generation antimicrobial agents. Most of the AMPs are composed of hydrophobic, hydrophilic and cationic residues arranged in a molecule that can organize into an amphipathic conformation to facilitate its interaction with bacterial cytoplasmic membrane. Recent studies on AMPs have led to increased understanding of their mechanisms of action and spectrum of activity. Along with isolating and modifying natural AMPs, designing peptide sequences de novo is becoming increasingly feasible to develop artificial AMPs. Unlike traditional antibiotics, AMPs due to their simiplicity offer significant flexibility for structural modification via amino acid substitution and many natural AMPs can be optimized with sequence engineering. There are several AMPs in the pipeline of clinical trials. And FDA approved gramicidin, a natural antimicrobial peptide (Figure 1.3), for treating topical infections. ${ }^{19}$ The development of AMPs indeed represents an important strategy in our fight against antibiotic resistance. 


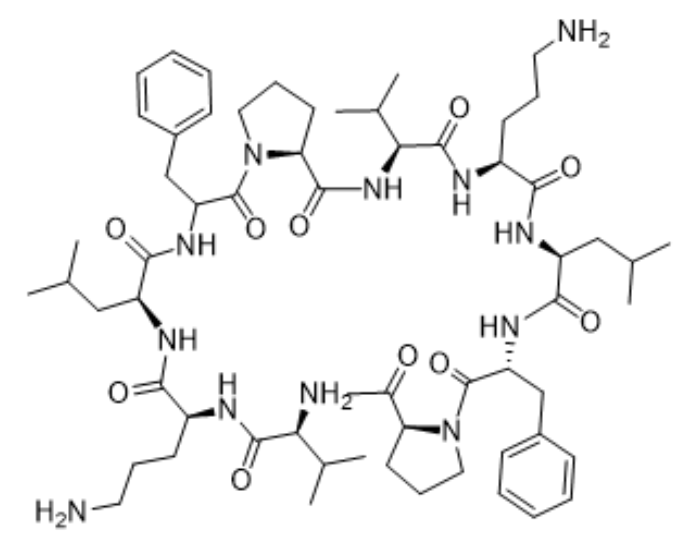

Figure 1.3 Structure of Gramicidin.

\subsection{Modification of existing antibiotics}

Synthetic chemistry offers unlimited possibilities in generating new bioactive compounds and analogs of existing antibiotics. Since the 1960s, the development of potent antibiotic analogs has been ongoing with many breakthroughs made along the way. After 1960s when discovery of new antibiotics becomes increasingly difficult, chemists started to develop semi-synthetic antibiotics which have proven efficacy. Many of these semisynthetic analogs are more potent and less prone to enzymatic degradation than the parent antibiotics while retaining the ability to interact with the same targets. The approach is safer than a traditional screening approach. Since 1962, medicinal chemists have reported the syntheses of tens of thousands of compounds, most being analogs of existing antibiotics and some being completely new synthetic antibiotics. Nalidixic acid was developed in 1962 as a synthetic antibiotic, and its modification has given rise to a new class of antibiotics. ${ }^{20}$ After 20 years of research on developing nalidixic acid analogs, a new family of broadspectrum fluoroquinolones was introduced to treat bacterial infections, which is among the most significant discoveries in antibiotic drug development through synthetic chemistry. There is no doubt that medicinal chemists have made great strides in developing new antibiotic analogs which address directly or indirectly the causes of drug resistance in 
resistant strains. Their work has broadened our understanding of different functional groups and their effect on pharmacokinetics and pharmacodynamics. ${ }^{21}$

Recently, the antibiotics used as the last resort in the treatment of critical conditions of bacterial infection, such as vancomycin, are increasingly becoming less effective due to emergence of resistant strains. ${ }^{22}$ One strategy to address this issue is again to modify those antibiotics. If chemical modification can revive their ability to kill drug-resistance microbes, it will be a significant achievement in the management of the difficult infections caused by these resistant strains. Antibiotics such as vancomycin that attack nonproteinaceous targets are known to pose a very high barrier for bacteria to develop resistance, as these non-proteinaceous targets are components which bacteria must maintain in their original form for the integrity of cellular structures. Vancomycin is a wellknown antibiotic which has been effective for three decades against drug-resistant infections because it targets a conserved peptidoglycan component of lipid II. Although bacterial strains resistant or less sensitive to vancomycin now exist, their emergence has been slow. So an attractive strategy is to develop new analogs of vancomycin, against which the development of resistance is also expected to be slow. The aim is to revive vancomycin's activity against resistant or less sensitive strains. Furthermore, if analogs are designed to have a dual mechanism of action by interfering with an additional bacterial function, they can maintain their efficacy for a much longer period by imposing a much higher barrier for resistance development. 


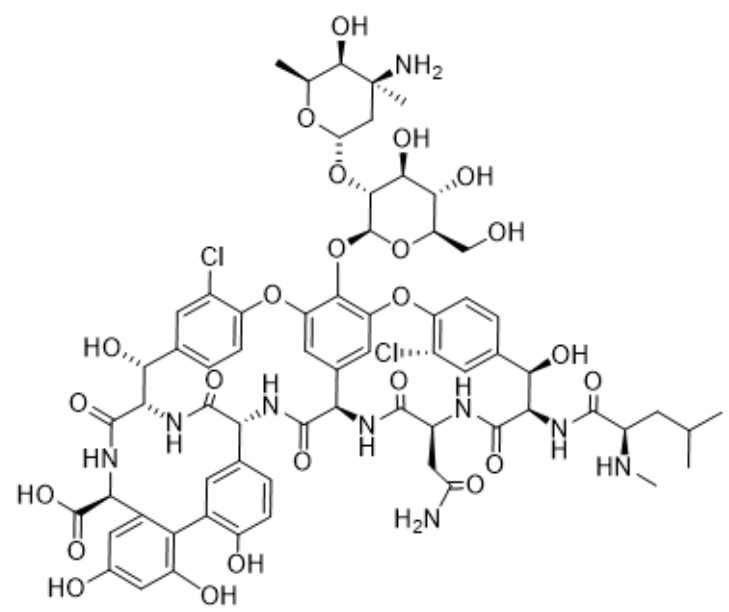

Figure 1.4 Structure of Vancomycin.

\subsection{Accessing the untapped resources}

Nature provides an abundant supply of diverse compounds. However, many of the natural compounds were previously considered as inaccessible. With advances in technology, we can now access some of these untapped resources. Recent advances in culturing previously uncultivable bacteria reveal that more than $99 \%$ of these microbial species is unexplored. Notably, the introduction of the IChip technology has made it feasible to grow bacteria in their natural habitat with significant yields. This technology is making it possible to explore the potential chemical space of natural products in millions of previously unexplored bacterial species. It has tapped a resource with enormous natural diversity. Using this technology, a novel antibiotic, teixobactin, has been discovered from soil microbes. ${ }^{23}$ The soil microbe from which the antibiotic was isolated was not known previously. The antibiotic seems to have an ability to evade drug resistance because it targets a conserved component of susceptible bacteria in multiple ways. The technology has opened a new avenue to discover novel antibiotics from the natural source. These new antibiotics, represented by teixobactin, as well as their potent analogs that are expected from synthetic chemistry, hold enormous promises in the battle against drug-resistant superbugs in the future. Teixobactin has a novel structure with a C-terminal macrolactone 
made up of four amino acid residues. As such it resembles the class of peptides known as cyclodepsipeptides.

Cyclodepsipeptides provide a rich source of potent therapeutic compounds. Several are reported to have cytotoxic, antiviral or antimicrobial properties. ${ }^{24}$ Kahalalide $\mathrm{F}$, extracted from a marine species of mollusk, exhibited potent anticancer activity and is under phase II clinical trial for prostrate cancer. ${ }^{25}$ Daptomycin is also a cyclodepsipeptide, and it is approved by FDA for the treatment of complicated skin infections. ${ }^{26}$ Given the success of the cyclodepsipeptides, teixobactin and its analogs hold great potential as a new class of antibiotics for the treatment of bacterial infections caused by the resistant strains, including the so-called superbugs.

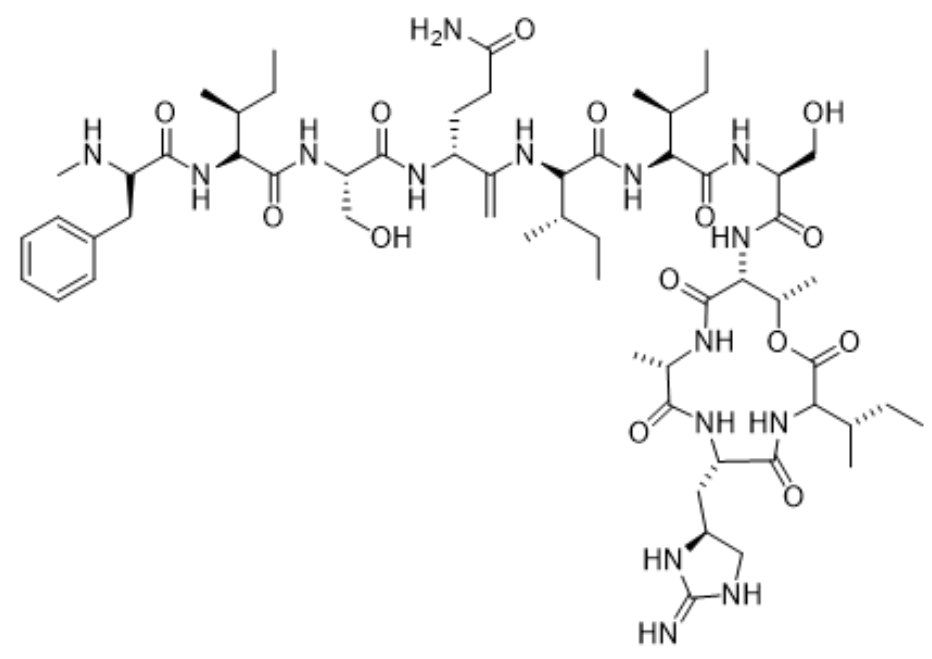

Figure 1.5 Structure of Teixobactin.

\subsection{Aims and significance of the study}

The majority of currently used antibiotics target essential enzymes or the protein synthesis machinery in the pathogenic microorganisms for their antibacterial effects. However, the barrier for the bacteria to develop resistance against these antibiotics is low because random mutations of the genes of these antibiotic targets can generate mutant 
strains that will be selected under the pressure of antibiotic exposure and eventually become predominant in the bacterial population. An effective strategy to evade or combat bacterial drug resistance is to find antibiotics that target bacterial sites which are difficult for bacteria to change or mutate. Antibacterial agents that target the bacterial membrane or cell-wall components are known to have such attributes. The cytoplasmic membranes are conserved domains of bacterial cells; yet they are considerably different from the mammaliam cell membranes in terms of composition, organization and plasticity, which makes it possible to achieve good selectivity. It is difficult for a bacterium to change its cell wall and many of its essential components. Therefore, targeting bacterial cytoplasmic membrane or cell-wall components would be more efficient in evading bacterial drug resistance or combating already resistant strains than targeting bacterial components that are directly encoded by DNA.

New potent antimicrobials with the ability to kill drug-resistant strains and prevent drug resistance are desperately needed to address the challenges in treating bacterial infections. To achieve the objective, novel approaches to enhance the capacity of existing antibacterial arsenal will be pragmatic and highly desirable, as these would offer a more practical solution to one of the biggest public health challenges of the $21^{\text {st }}$ century.

The aim of my thesis is to design new potent peptide-based antimicrobial agents through modification of existing antimicrobials to enhance their efficacy. The studies are focused on antimicrobials that are known to target bacterial cytoplasmic membrane or cell wall. In this context, I explored three main strategies which are as follow.

- Dimerization of membrane-active antimicrobial peptides (AMPs) to enhance their stability and antibacterial activity.

- Modifying an existing glycopeptide antibiotic, i.e. vancomycin, by preparing its C-terminal amide derivatives to improve its antibacterial activity.

- Developing an efficient method for synthesizing analogs of teixobactin, a new cyclodepsipeptide antibiotic active against various resistant strains. 
Membrane-active AMPs are considered as next-generation antibiotics. Examples of AMPs are polymyxin, colistin, gramicidins, and daptomycin. Optimization of the AMPs can derive very potential antimicrobials against the resistant strains. Some AMPs are going through various stages of clinical trials. ${ }^{27}$ It is known that dimerization can enhance the stability and antibacterial activity of certain AMPs, potentially making them superior antibiotic candidates. The dimers are usually prepared with a traditional method of solid phase synthesis. In the study reported in Chapter 2, an efficient method for peptide dimerization is developed and tested on two different AMPs of either hydrophilic or hydrophobic nature, which has generated very different outcomes.

Another effective strategy to enhance the activity of existing antibiotics is through modifying their structures at predetermined positions. In this study, one of the most potent glycopeptide antibiotics, vancomycin, was chosen as a target compound for structural modification. Vancomycin targets Lipid II of bacterial cell wall. For almost five decades, it has been the last resort and the antibiotic of choice for drug-resistant infections until recently. Bacterial drug resistance is making vancomycin increasingly ineffective. Therefore, developing more effective analogs of vancomycin is in great need. In this thesis, a simple and highly effective approach to develop potent vancomycin analogs is presented in Chapter 3. This approach should also be applicable to other Lipid II cell-wall targeting glycopeptide antibiotics, such as daptomycin, oritavancin and telavancin which form the arsenal of the last resort against bacterial drug resistance. ${ }^{28}$

Recently, a breakthrough was achieved with the discovery of teixobactin. ${ }^{23}$ This game-changing antibiotic also targets Lipid II but in multiple sites of the target to inhibit bacterial cell wall synthesis. With its unique mechanism of action, teixobactin can effectively evade bacterial drug resistance. ${ }^{23} \mathrm{~A}$ new scheme is designed and presented in Chapter 4 of this thesis for the efficient synthesis of teixobactin analogs. The methodology presented can expedite the discovery of various potent teixobactin analogs and thereby enhance our ability in the fight against the problem of bacterial resistance. 


\section{CHAPTER 2 \\ PREPARING DIMERS OF MEMBRANE- ACTIVE ANTIMICROBIAL PEPTIDES}

\subsection{Introduction}

\subsubsection{History}

Antimicrobial peptides (AMP) are a conserved evolutionary component of the innate immune system of most organisms. The first reported AMPs were identified in prokaryotic cells in 1939 when the antimicrobial substances, gramicidins, were separated from Bacillus brevis. Gramicidins were seen to be effective against numerous gram positive and gram negative bacteria both in vivo and in vitro. ${ }^{29}$ In 1942, an antimicrobial substance was revealed in wheat flour ${ }^{30}$ that was separated from wheat endosperm (Triticum aestivum) and detected to be a peptide that restricted the growth of numerous phytopathogens, such as Pseudomonas solanacearum and Xanthomonas campestris. ${ }^{31}$ In the mid-1970s this peptide was named as purothionin, ${ }^{31}$ and it belongs to the family of thionins. Generally, AMPs comprise of less than 100 amino acid residues. Many active AMPs are composed of hydrophobic, hydrophilic and cationic residues arranged in a molecule that can organize into an amphipathic conformation. ${ }^{32}$

\subsubsection{Importance of antimicrobial peptides}

The discovery of antibiotics was a major milestone in our fight against infectious diseases. However, the continuous emergence of resistant strains poses serious challenges to the treatment of infectious diseases caused by bacteria. 
There is great need to introduce new molecules with novel antibacterial mechanisms to address the problem of drug resistance. AMPs are an attractive alternative to conventional antibiotics. They have broad-spectrum antimicrobial activities against microbes. They are effective against gram-negative and gram-positive bacteria, fungi, protozoa and viruses with micromolar to submicromolar minimum inhibitory concentration (MIC). Most AMPs can effectively kill multi-drug resistant (MDR) bacteria. AMPs possess low predilection for resistance development, can be selective in binding to their targets and hence pose low toxicity problem, and are capable of synergizing with existing antibiotics. ${ }^{33}$

\subsubsection{Diversity of AMPs and structural classification}

Antimicrobial peptides are structurally very diverse. Therefore, segregating them on a stringent basis is difficult. Nevertheless, they can be classified by their structure. ${ }^{34-35}$

Most AMPs, after coming in contact with membrane lipids, orient themselves from their water-soluble unstructured state to a more amphipathic structured conformation. Based on the composition, size, conformation and structure, AMPs can be classified as: ${ }^{33}$

- $\alpha$-Helical peptide, e.g., magainin, cecropin, cathelicidins

- $\quad \beta$-Sheet peptides stabilized with disulfide bonds, e.g., $\beta$-defensin

- Extended helix peptides, e.g., indolicidin

- Loop peptides, e.g., cyclic defensins

\subsubsection{Mechanism of action}

The molecular mechanism underlying the antibacterial activity of AMPs is not completely understood, except for the part that positively charged groups on AMPs can be bonded to the negatively charged lipid head groups on the outer leaflet of the microbial membrane. This interaction is followed by drastic changes in the bacterial membrane 
structures. AMPs may reorient itself on the membrane or translocate through the membrane to reach intracellular targets. ${ }^{36}$ Numerous parameters such as physiochemical property, size, hydrophobicity, secondary structure and amphiphilic character determine the functionality of membrane penetration and bisection. ${ }^{37}$ Therefore, AMPs are divided into two mechanistic classes:

- Non-membrane bisection class - these are AMPs that attack intracellular targets.

- Membrane bisection class - these are AMPs that exhibit barrel stave, toroidal, carpet and micellar aggregate models.

No matter which class, the AMPs would need to interact with the microbial membrane. Different models have been proposed for AMP membrane permeabilization (Figure 2.1). Although consensus has not reached on any of these models.

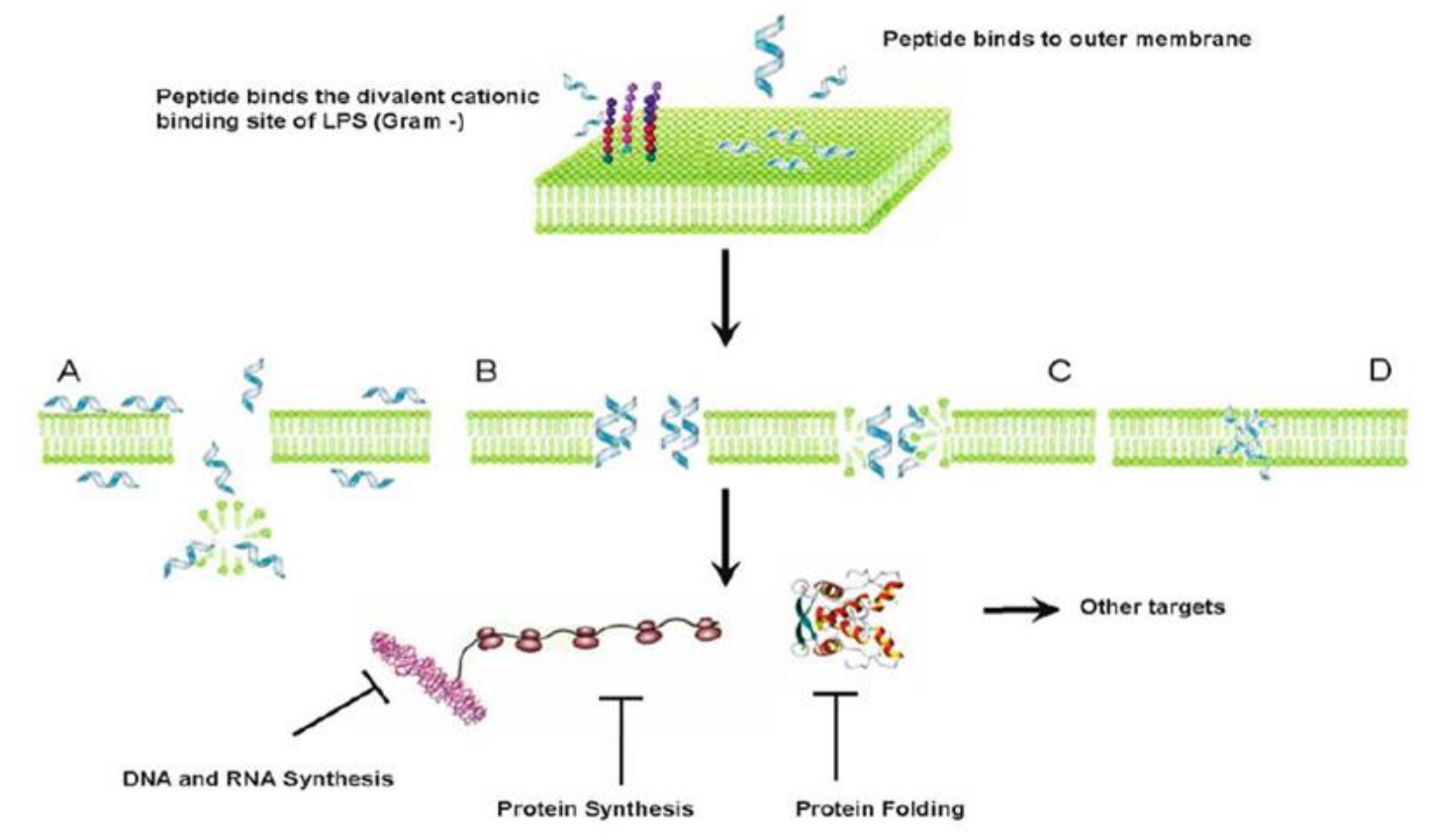

Figure 2.1 The listed models: (A) carpet, (B) barrel-stave, (C) wormhole or toroidal, and (D) aggregate channel. The net effect of A to D is that some monomers will be translocated into the cytoplasm and can dissociate from the membrane and bind to cellular polyanions such as DNA and RNA, inhibit enzymatic activity such as protein synthesis or chaperone assisted protein folding. ${ }^{38}$ 


\subsubsection{Design of synthetic AMPs}

Recent studies on the basic characteristic features of existing AMPs and their computational analysis have provided insights which lead to the effective design of novel AMPs. These methods utilize a database or source which has a constellation of known or predicted peptide sequences. ${ }^{39}$ New AMPs can also be designed using others means, such as making dendrimeric peptides and $\mathrm{AB}$ initio design. ${ }^{40}$

Structurally, certain AMPs exist as a beta sheet while others in alpha helical conformation. It is possible to attain increased potency of existing AMPs by sequence engineering and dimerization. ${ }^{41}$ In this chapter, two AMPs with very different properties were chosen in my study of preparing their dimers: one is a hydrophobic peptide with an alpha helical conformation originally obtained through $\mathrm{AB}$ Initio design and the other a highly cationic hydrophilic peptide derived from a human beta defensin which is predominantly made of beta sheets.

\subsubsection{AB Initio design}

It is a novel way of designing synthetic antimicrobial peptides. ${ }^{40}$ The antimicrobial peptide database is used to design a peptide based on most probable parameters derived from natural AMPs. The database contains key information of 2208 AMPs (Dept of Pathology and Microbiology UNMC 2013) as of April 2013. A database filtering technology is used for this purpose. The AMPs in the database reported to have activity against gram positive bacteria are considered as the first filter. This set was used as a model to derive most probable parameters for peptide design. Peptide length is the second filter. The peptide length is a dominant factor among most of the AMPs. The third filter determines which amino acids to be used. A fourth filter determines the number of charged residues. Besides cationic residues, hydrophobic residues equally play an essential role. Hydrophobicity is considered as the fifth filter. Another filter is the structural distribution of peptides. In most cases, helical conformation for the designed peptides is preferred. ${ }^{40}$ 
According to this method, an additional motif can be added to aid the peptide in getting the desired conformation. Using $\mathrm{Ab}$ initio design, potent peptides were designed and synthesized, one being DFTamP1-P which is effective against MRSA. ${ }^{40}$ The broad hydrophobicity is essential for the antibacterial activity of DFTamP1-P, as its hydrophobic region assists the peptide penetration across the membrane. ${ }^{40}$

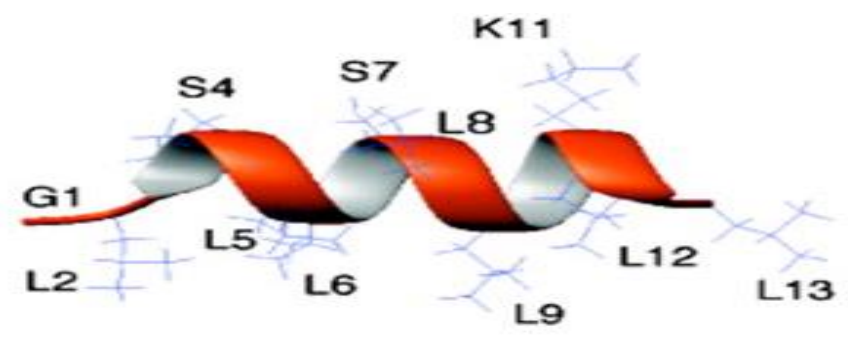

Figure 2.2 Ribbon model representation of DFTamP1-p (peptide sequence GLLPLLSLLGKLL). The picture was taken from Wang et al. ${ }^{40}$

\subsubsection{Dendrimeric peptides}

Dendrimeric peptides are biopolymers with unconventional properties. Tam et al. ${ }^{42}$ introduced dendrimeric antimicrobial peptides built on a multi-lysine scaffold. They exploited the polyvalency of a dendrimer through tethering peptides to a branching lysine core, which enhances the interaction with the lipid bilayer of the microbe. In their study, the designed dendrimeric peptides exhibit high potency against ten bacterial strains, in conditions of high and low salt concentrations. ${ }^{42}$ 


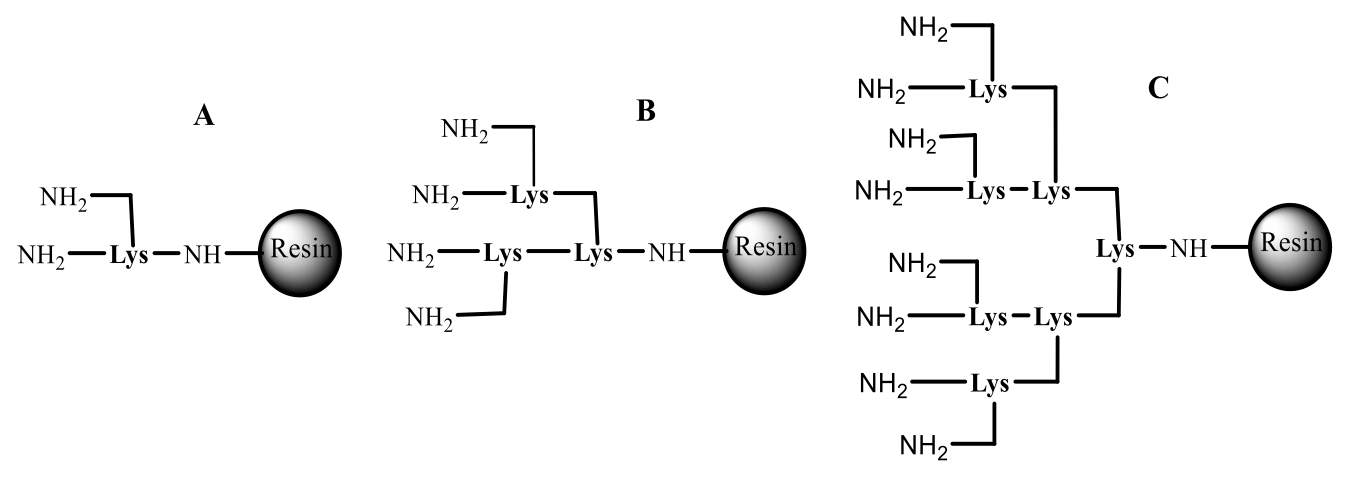

Figure 2.3 Three types of dendrimeric cores with three generations of lysines, A. Two branched Lys; $\mathbf{B}$. Four branched (Lys) $)_{2}$ Lys: C. eight-branched $\left[(\operatorname{Lys})_{2} \text { Lys }\right]_{2}$ Lys. Picture was taken from James et al. ${ }^{42-43}$

Like designing the dendrimers, ${ }^{42}$ Bracci et al ${ }^{44}$ proposed that synthesizing dimers of bioactive peptides could increase their half-life and resistance to proteases and peptidases. As a result, the dimeric form would also have higher in vivo efficacy than the monomer. This hypothesis was successfully verified by testing the stability of monomeric and dimeric peptides in human plasma and serum. ${ }^{45}$ Therefore, synthesizing dimeric peptides of AMPs could be helpful in developing potent antimicrobial compounds. Some of the human beta defensins peptides naturally exist as dimers. They can be used to design and synthesize the dimeric peptides.

\subsubsection{Human beta-defensins}

Human beta-defensins are ideal candidates to develop potent dimerized AMPs. Human defensins comprise the largest family of AMPs in humans. It has three subfamilies, i.e., $\alpha$, $\beta$ and $\Theta$ defensins. $\beta$-defensins are considered to be more potent in its antimicrobial activity. In the literature, six subtypes of human Beta-defensin (HBD) are reported (HBD 1-6). Numerous studies have reported their distinctive properties, making them particularly potential antimicrobial agents. Among the subtypes, HBD-3 is the most potent. HBD-3 also stays in non-epithelial tissues like placenta, tonsils, adult heart, skeletal muscle and fetal thymus. ${ }^{46}$ 


\subsubsection{3(i) Structure of HBD-3}

HBD-3 is a peptide of 45 amino-acid residues having a molecular mass of $5.15 \mathrm{kDa}$, containing a six-Cys motif linked at the 1-6, 2-4 and 3-5 positions to form three disulfidebridges. ${ }^{47}$ HBD-3 has a particularly high net positive charge. In its amphiphilic structure, hydrophobic residues are aggregated at the bottom while positive charges are on the two sides of the surface.

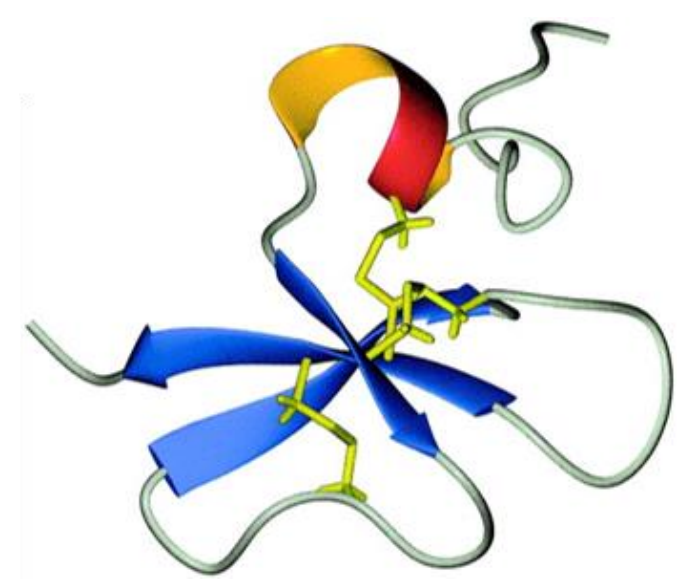

Figure 2.4 Ribbon diagram structure of HBD-3. The three disulfide-bonds are shown in gold. ${ }^{48}$ HBD-3 sequence: GIINNTLQKYYCRVRGGRCAVLSCLPKEEQIGKCSTRGRKCCRRKK

$\beta$-Defensins exert their antimicrobial activity by binding the bacterial surface via electrostatic interactions and enter into the membrane by hydrophobic interactions. ${ }^{49}$ Klüver et al. examined the antibacterial mechanism of synthetic analogs of HBD-3 having variations in charge, length, disulfide connectivity and overall hydrophobicity. ${ }^{50}$ They showed that peptides with similar secondary structure have different antimicrobial potential, observing that the antimicrobial effect of HBD-3 is strongly dependent on the distribution of positively charged amino acid residues and hydrophobic side chains. ${ }^{50}$ 


\subsubsection{3(ii) Biological function and structure-activity relationship}

Some in vitro studies have reported antibacterial, antifungal and antiviral activities of HBD-3. ${ }^{46} 515253$ HBD-3 displays various antibacterial effects against Gram-positive and Gram-negative bacteria at lower concentrations in comparison to other members of the $\beta$ defensin family. ${ }^{52}$ Batoni et al. found that HBD-3 had bactericidal activity toward most of MDR (Multidrug resistant) nosocomial pathogens. ${ }^{53}$ This is fascinating as HBD-3 exhibited bactericidal activity against the tested clinical isolates, irrespective of their resistance profile. ${ }^{54}$ It has been shown that antimicrobial cationic peptides exhibit rapid killing kinetics than the commonly used antibiotics. ${ }^{33}$ Impressively, HBD-3 displayed a very fast bactericidal mechanism against gram negative and gram positive bacteria within 1-5 min and 10-20 min, respectively. Interestingly, the cysteine residues and disulfide bonding do not seem to be important to the antimicrobial activity of HBD-3. ${ }^{50,55}$ Moreover, the N-terminal extension of five amino acids has not much effects in antimicrobial activity of HBD-3 peptides, while peptide extension at the C-terminal significantly alters its activities. $^{50,55}$

AMPs, when administered together with bactericidal antibiotics, should have enhanced antibacterial action. Studies were conducted to verify the synergistic relationship between HBD-3 and antibiotics. The co-administration of suboptimal doses of $\beta$-lactam antibiotics, such as methicillin, with HBD-3 had co-active consequence against methicillinsensitive and resistant $S$. aureus (MSSA and MRSA). A comparable effect was observed with metronidazole, amoxicillin and oral disinfectant chlorhexidine. ${ }^{52}$ Periodontal pathogens (A. actinomycetemcomitans and $P$. gingivitis) and cariogenic species ( $S$. mutants) were more sensitive to the synergistic action of AMP and antibiotics than the AMP alone. ${ }^{52}$ It is suggested that the drugs that can cause a breakdown of the bacterial cell wall (i.e. amoxicillin, methicillin, lysozyme) might promote the access of HBD-3 into the cytoplasmic membrane. Such a hypothesis is consistent with the observation that, when $S$. aureus was exposed to suboptimal concentrations of methicillin (1/16 MIC), the bacterial cells became more vulnerable to HBD-3 than untreated bacteria. ${ }^{56}$ Simultaneously, the 
excessive permeability of membranes caused by the peptide could facilitate the entry of antibiotics (i.e. metronidazole, chlorhexidine) with an intracytoplasmic target.

The negative charge on the bacterial membrane is important for the antimicrobial mechanism of defensins, including HBD-3. It was reported that mutating mprF and lysC genes of Staphylococcus Aureus reduces lysyl-phosphatidylglycerol inside the cell membrane which leads to greater net negative charge, resulting increased susceptibility to HBD-3. ${ }^{57,} 58$

\subsubsection{3(iii) HBD3-derived small peptides and importance of dimerization}

To identify HBD-3 fragments responsible for the antimicrobial activity, Liu et al. synthesized and tested different peptide fragments of HBD-3 taken from the C-terminal region of HBD-3. The effective peptide they found was RGRKVVRRKK. ${ }^{59}$ A branched V2-dimer (RGRKVVRR) ${ }_{2} \mathrm{KK}$ was created, in which two copies of RGRKVVRR are joined to the $\alpha$ and $\varepsilon$ amines of a lysine residue. ${ }^{59}$ Their study reported the V2-dimer as a more potent antibacterial entity than the monomer. This series of 10-residue C-terminal peptides and their V2-dimers showed the least toxicity to mammalian cells. The literature has shown that charge density, stable covalent dimerization and adopting well-defined structure are important parameters for effective cationic antimicrobial peptides. ${ }^{59}$

Different studies have shown that various natural peptides undergo selfdimerization before insertion into the microbial membrane. NMR studies have revealed that pexiganan adopts a helical configuration while interacting with membrane and its dimeric anti-parallel $\alpha$-helical structure is inserted into the membrane. ${ }^{60}$ Halocidin is an AMP that exists naturally as a dimer. ${ }^{61}$ Dimerization brings many pharmacokinetic and pharmacodynamic advantages to AMPs. However, dimerization is not a universal phenomenon; there are natural AMPs existing as monomers and exhibit their antibacterial activity. Human beta defensin-2 is another AMP member from the defensins family. But it is active in monomeric form. ${ }^{62}$ On the contrary, HBD-3 exhibits bactericidal activity as a dimer. 


\subsubsection{Dimerization of HBD-3 peptide and DFTamP1-p in this study}

As discussed above, dimerization of the highly cationic and hydrophilic HBD-3 peptide significantly increases the antibacterial activity. However, dimerization and its

effect on hydrophobic AMPs are not extensively studied. These hydrophobic AMPs mostly adopt alpha helical conformations and one such peptide is the $\mathrm{AB}$ Initio designed DFTamP1-p as mentioned previously. ${ }^{40}$ It is therefore desirable to also investigate the effect of dimerization on the secondary structure and antibacterial activity of such hydrophobic AMPs.

Peptides dimers are usually synthesized using traditional stepwise solid phase peptide synthesis techniques. However, these methods have limitations regarding the efficiency, low yield, and difficulty in synthesizing dimers with long peptide chains. Therefore, an alternative way of peptide dimerization is desired. In this project, we chose to use a convergent chemical conjugation method for peptide dimerization. In this method, the monomeric peptides are synthesized and purified to homogeneity before being joined together to form dimers by a simple conjugation reaction. The conjugation reaction is a classical nucleophilic substitution reaction of $\mathrm{SN}_{2}$ type between a thiol group and a bromoacetyl moiety, which forms a covalent thioether linkage. This method is useful for dimerizing the AMPs.

In this study, two different peptides are chosen for dimerization studies using the convergent method. One is hydrophobic and the other is hydrophilic. The hydrophobic peptide is the above-mentioned DFTamP1-p obtained through AB initio design and reported to adopt an alpha helical conformation; ${ }^{40}$ the hydrophilic peptide is the cationic hydrophilic peptide derived from HBD-3 is also used by Liu et al. ${ }^{59}$ which presumably is active in $\beta$-sheet conformation. It will be interesting to know the outcome of dimerization as to whether it will have different effects on these two peptides with different conformations. Such a study will provide useful information for the design of new AMPs to fight drug-resistant bacterial strains. 


\subsection{Results and discussion}

It has been established that the dimeric forms of certain bioactive peptides manifest high potency in antimicrobial assays. ${ }^{59}$ Many peptides, including pexiganan and human beta defensin-3, exist naturally as monomers but adopt dimeric conformation after interaction with bacterial membrane, which makes them more potent. ${ }^{60}$ Many studies have disclosed that upon interaction with the lipid membrane, peptide forms a distinct structure that tends to condense the positive charges within peptide oligomers. The charge density, mediated by corresponding 3D structures, is directly correlated with the antimicrobial activity. ${ }^{59}$ The peptide dimers exhibit greater in vivo efficiency than monomeric form due to pharmacokinetic advantages, such as increased half-life, resistance to peptidase, prolonged renal clearance and protease stability. Similarly, dimeric forms of these peptides increase their antimicrobial activity in high and low salt concentrations. Therefore, it can be concluded that the dimeric form of an antimicrobial peptide is often more effective and stable.

In solid phase peptide synthesis (SPPS) of peptide dimers, a lysine core structure is usually utilized which possesses $\alpha$ and $\varepsilon$ amine groups. The peptide chain is then extended by simultaneous coupling of amino acids on both amine groups until the full sequence is completed. SPPS is efficient to synthesize linear monomeric peptides but may be less efficient for peptide dimer synthesis especially when the peptide chains are long. Aggregation between peptide chains, tedious and repetitive re-coupling of amino acids and significant reagent usage are additional limitations of synthesizing peptide dimers by SPPS. Therefore, a modular approach to peptide dimerization through thiol alkylation is proposed in this study.

\subsubsection{Different ways of dimerization}

Considering the limitations of the conventional methods, we utilized a different approach whereby monomeric peptides were first prepared by SPPS and joined to a core 
or another peptide monomer by a simple conjugation reaction. This reaction can be used for making dimers or conjugating different peptides. In our study, this method is used to make dimers of HBD-3 and DFTamP1.

\subsubsection{Thiol alkylation-mediated dimerization}

Thiol alkylation involves a reaction between a thiol group of one peptide and a bromoacetyl moiety of another peptide. ${ }^{63}$ The nucleophilic substitution reaction forms a covalent thioester linkage joining the two peptides. This principle is used for all the thiol alkylation-mediated dimerization reactions in this study.

Thiol alkylation is a simple way to synthesize peptide dimers or conjugate different peptides together in a single reaction step. The reaction requires alkaline $\mathrm{pH}$. In our study, sodium bicarbonate was used to adjust the $\mathrm{pH}$ to about 8 . All the reactions were conducted at room temperature. ${ }^{63}$

The general scheme for thiol alkylation-mediated conjugation/dimerization is shown below. 


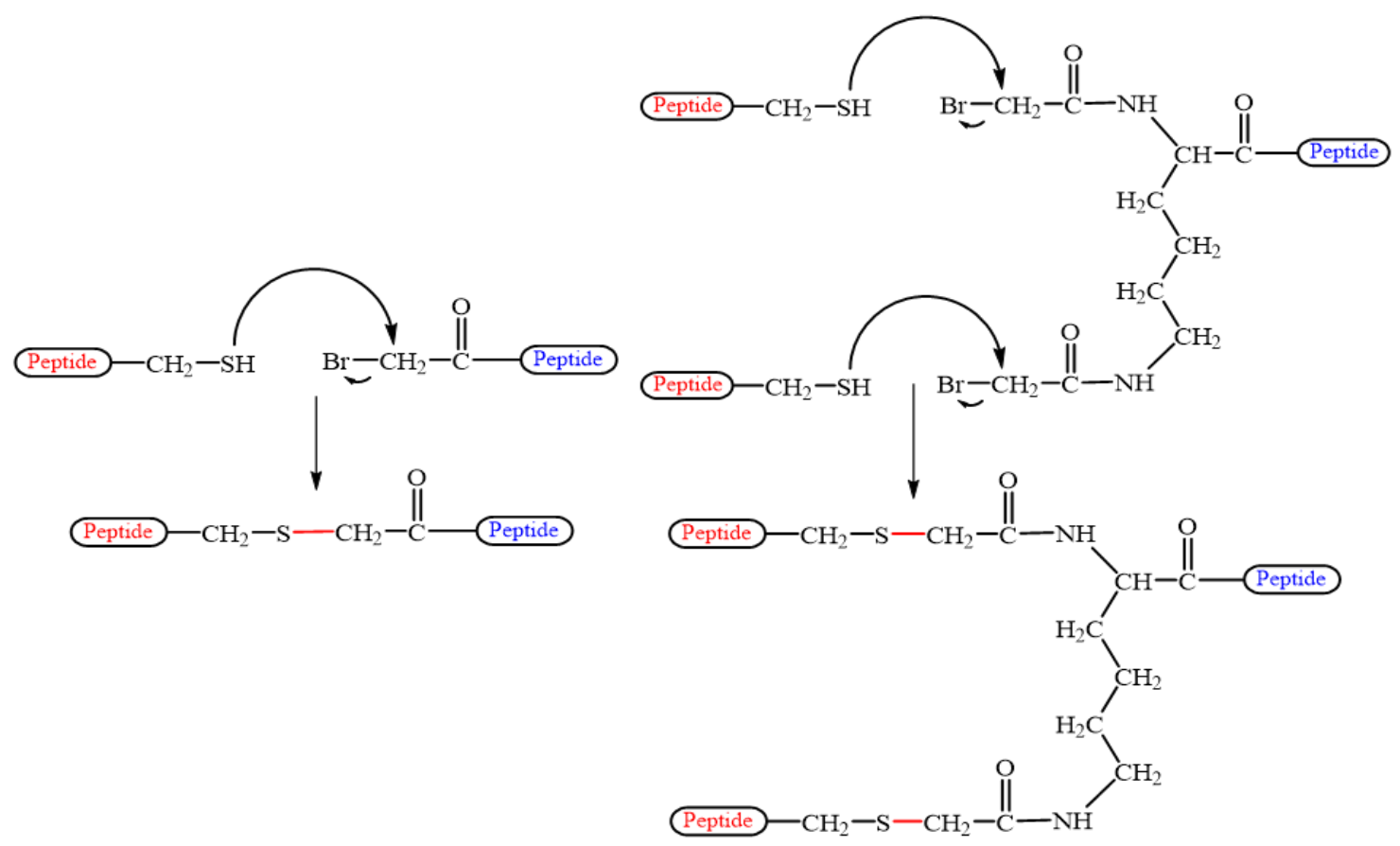

Figure 2.5 General scheme for thiol alkylation. Panel left: peptide-peptide conjugation; Panel right: peptide dimerization on a bivalent core.

We used SPPS for the synthesis of monomeric peptides and the traditional Lysbased dimers, ${ }^{42}$ whereas thiol alkylation in Figure 2.5 (right panel) was used to synthesize most of the dimeric variants. After synthesizing the required peptides, bioassays were conducted to determine the antibacterial activities.

\subsubsection{HBD-3-derived V2 peptides and their dimerized versions}

Liu et al. ${ }^{59}$ reported a peptide fragment taken from the C-terminal part of HBD-3 with the sequence H-Arg-Gly-Arg-Lys-Val-Val-Arg-Arg-Lys-Lys- $\mathrm{NH}_{2}$. The peptide has modest antimicrobial activity and is termed as a V2 peptide. It is considered as an analog of human beta defensin-3. The dimeric form of the V2 peptide exhibits higher antimicrobial activity, and the peptide is known as a V2 dimer. It was synthesized by linking two peptide chains of RGRKVVRR to a lysine core residue. V2 dimer was synthesized on $0.2 \mathrm{mmol}$ rink amide resin and the final yield was approximately $30 \%$ of the crude peptide. The 
synthesis of dimer consumes significantly more reagents. The V2 monomer and V2 dimer peptides were synthesized and used as controls in our study. Figure 2.6 shows the V2 dimer.

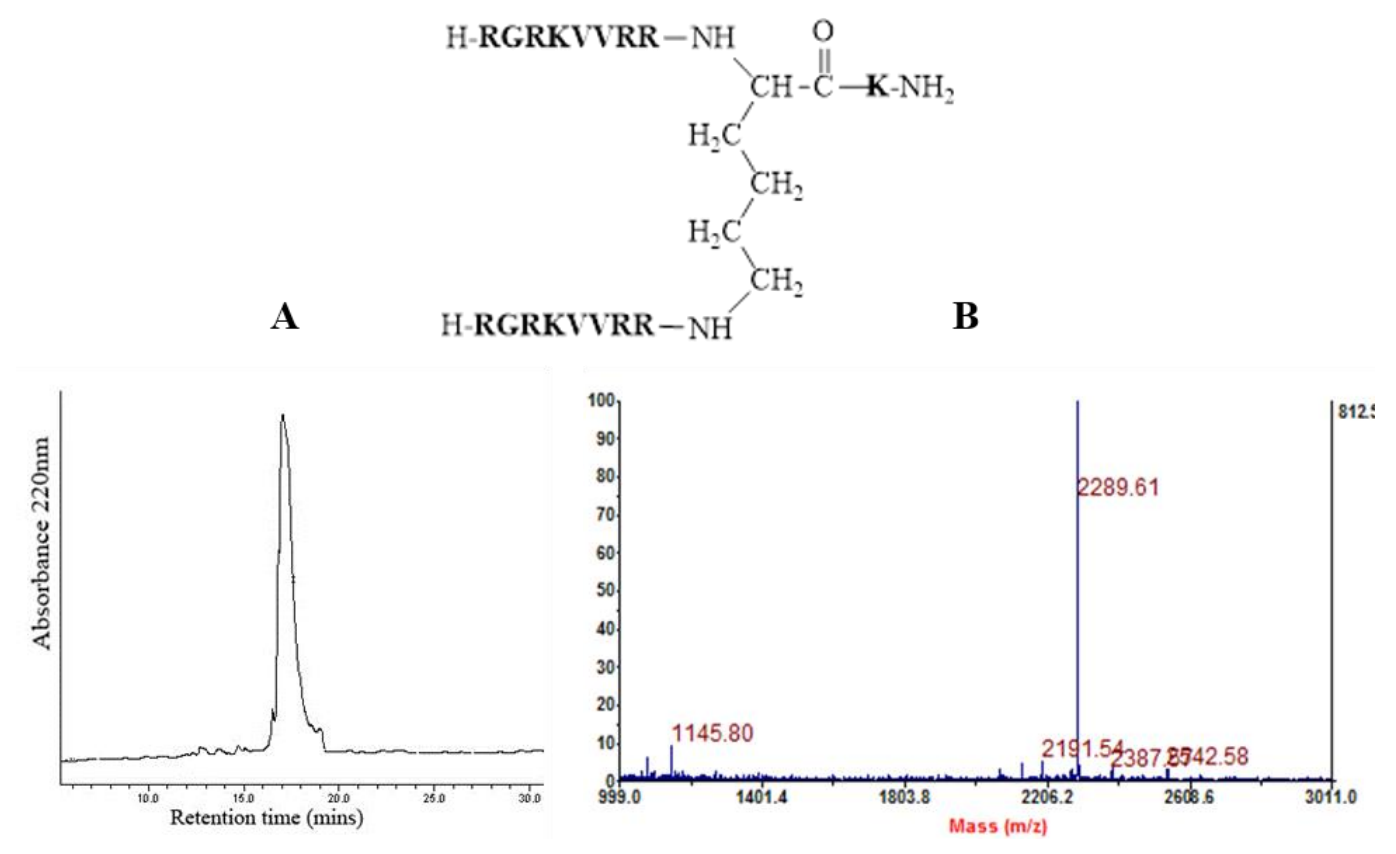

Figure 2.6 A. C18 analytical HPLC profile of the pure V2 dimer. Gradient: buffer B 0-40\% in $40 \mathrm{~min}$ B. MALDI-TOF MS of purified V2 dimer. [M+H $\left.{ }^{+}\right]^{+} \mathrm{m} / \mathrm{z}=2289.61$ (found), 2287.92 (calculated).

The V2 dimer has potent antimicrobial activity against several microbes as reported in the literature. ${ }^{59}$ It was directly synthesized by SPPS using the traditional approach of tethering peptide chains on the bi-functional lysine. The structure of V2 dimer is shown in Figure 2.6. Figure 2.6 A and B shows the HPLC profile and MALDI-MS of purified V2 dimer peptide.

We have prepared several thiol-containing V2 peptides which were then alkylated with a di-bromoacetyl lysinyl moiety for dimer formation. As seen from Table 2.2, RGRKVVRR was used as a template to design different dimeric derivatives by thiol alkylation. The dimerization reaction requires monomer derivatives possessing a thiol moiety. In all cases, the thiol group was placed at the $\mathrm{C}$-terminus of the peptide. The first derivative synthesized was V2-AGC which has the V2 template sequence with a spacer AG. 
Table 2.1 List of all V2-related peptides synthesized in this study.

\begin{tabular}{|c|c|c|}
\hline No & Sequence of peptide & Name of peptide \\
\hline 1 & H-RGRKVVRR-NH ${ }_{2}$ & V2 monomer \\
\hline 2 & H-RGRKVVRRAGC-NH ${ }_{2}$ & V2-AGC \\
\hline 3 & $(\text { H-RGRKVVRR })_{2} \mathbf{K K}-\mathrm{NH}_{2}$ & V2 dimer \\
\hline 4 & H-RGRKVVRR- $\mathrm{NHCH}_{2} \mathrm{CH}_{2} \mathrm{SH}$ & V2-Cya \\
\hline 5 & H-RGRKVVRRC-NH 2 & V2-Cys \\
\hline 6 & $\left(\text { H-RGRKVVRRAGC-NH }{ }_{2}\right)_{2}^{K K-\mathrm{NH}_{2}}$ & V2-AGC dimer \\
\hline 7 & $\left(\mathrm{H} \text {-RGRKVVRRC-NH}{ }_{2}\right)_{2}^{\text {KK-NH }}$ & V2-Cys dimer \\
\hline 8 & (H-RGRKVVRR- $\left.\mathrm{NHCH}_{2} \mathrm{CH}_{2} \mathrm{~S}-\mathrm{CH}_{2} \mathrm{CO}\right)_{2} \mathbf{K K}-\mathrm{NH}_{2}$ & V2-Cya dimer \\
\hline 9 & $\left(\mathrm{BrCH}_{2} \mathrm{CO}-\right)_{2} \mathbf{K K}-\mathrm{NH}_{2}$ & Di-bromoacetyl-Lys core \\
\hline
\end{tabular}

Bold letters denote amino acids. Red color denotes thiol containing moieties.

Antibacterial activity of most AMPs depends on its sequence, conformation and amphiphilic nature. ${ }^{64}$ Kluber et al. examined the antibacterial potential of numerous synthetic analogs of HBD-3 that were different in charge, length, disulfide interaction and overall hydrophobicity. Interestingly, they observed that peptides having the same secondary structures exhibited significant variations in their antimicrobial potency, suggesting that the antimicrobial effects of HBD-3 mostly depend on the peptide sequence rather than on the secondary structure ${ }^{50}$ Hence, it is possible that the presence of a spacer might itself alter the activity of the whole peptide. Therefore, we also synthesized other derivatives of the V2 peptide template without a C-ter spacer and compared the antibacterial activity of these derivatives.

All the V2 monomers were synthesized by Fmoc solid phase peptide synthesis. As an example, the first sequence designed, H-Arg-Gly-Arg-Lys-Val-Val-Arg-Arg-Ala-Gly- 
Cys- $\mathrm{NH}_{2}$, was prepared using Rink amide MBHA resin. The peptide resin was cleaved in the TFA cocktail solution for 1 hour. After peptide precipitation using cold ether, the crude peptide obtained was analyzed by analytical HPLC, followed by its purification by semipreparative HPLC. The yield of purified peptide was approximately $50 \%$ of the crude peptide. Figure 2.7 shows the analytical HPLC profile of the pure peptide and its MALDITOF MS.

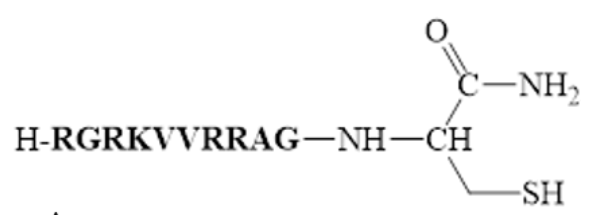

$\mathbf{A}$
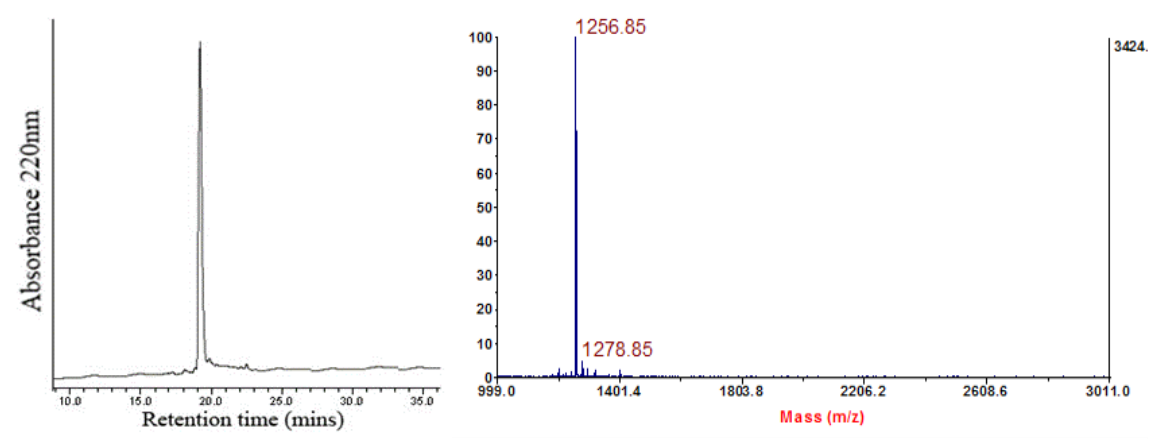

Figure 2.7 A. C18 analytical HPLC profile of pure V2-AGC, Gradient buffer B 0-40\% in 40 mins B. MALDI-TOF MS of V2-AGC $\left[\mathrm{M}+\mathrm{H}^{+}\right]^{+} \mathrm{m} / \mathrm{z}=1256.85$ (observed), 1256.55(calculated).

To determine whether the addition of a C-ter spacer affected antibacterial activity, the minimum inhibitory concentration (MIC) values of the V2-AGC on two bacteria were first assessed by the sensitive two-stage radial diffusion assay developed by Lehrer et al. ${ }^{65}$ In the first stage, log-phase bacteria were mixed with molten nutrient-poor underlay agarose gel and solidified into Petri dishes. Agarose was used instead of normal agar as it minimizes electrostatic interaction between peptides and the gel. Small wells $(2 \mathrm{~mm}$ in diameter) were punched on the gel, and peptide samples of different concentrations were added. The peptides diffused into the gel in a radial manner, resulting in inhibition zones around the wells if the peptides possessed antimicrobial activity. In the second stage, the clear zones were visualized by pouring another nutrient-rich layer of gel on top of the underlay, which allowed surviving bacteria to grow. For each particular peptide, the sizes 
of its clear zones correlated linearly with its log of concentration, i.e. higher concentrations gave rise to bigger clear zones. Consequently, the MICs were determined from diameters of zone of inhibition and $\log$ [concentration] graphs by calculating the $\mathrm{x}$-intercept of the fitted line. The results are shown in Table 2.2.

Table 2.2. MIC of V2 monomer and V2-AGC peptide.

\begin{tabular}{lcc}
\hline Name & V2 & V2-AGC \\
Sequence & H-RGRKVVRRKK-NH 2 & H-RGRKVVRRAGC-NH 2 \\
\hline Escherichia coli & 39 & 18 \\
Pseudomonas aeroginosa & $>39$ & 7 \\
\hline
\end{tabular}

The radial diffusion assays showed that V2-AGC retained antibacterial activity. It appears that adding Ala-Gly-Cys to the C-terminus did not negatively influence V2-AGC activity. V2-AGC was found to be active against Gram-negative bacteria Escherichia coli with MIC of $18 \mu \mathrm{M}$. Likewise, it inhibited the growth of pathogenic Pseudomonas aeroginosa with MIC of $7 \mu \mathrm{M}$, which is better than the $\mathrm{V} 2$ monomer peptide, suggesting V2-AGC has a slightly different MIC profile than the V2 monomer. The exact reason for this was not further investigated. Plausible reasons can be a change in peptide conformation after adding the $\mathrm{C}$-terminal AGC tripeptide. Alternatively, oxidation of the cysteine residue in V2-AGC during the bioassay may lead to the formation of the disulfide-linked dimer which has higher antibacterial activity, although the analytical HPLC of V2-AGC peptide conducted prior to the assay showed the absence of any oxidized product.

\subsubsection{The bivalent linker for V2 peptide dimerization - di-bromoacetyl- Lys-Lys- $\mathrm{NH}_{2}$}

We aimed to synthesize V2 dimers using a convergent approach whereby the linear peptides synthesized by SPPS are joined together with a bivalent linker using the thiol alkylation reaction. The bivalent linker is di-bromoacetyl-Lys- $\mathrm{Lys}^{-\mathrm{NH}_{2}}$ as shown in Figure 
2.8 A. So a bromoacetyl moiety is attached to $\alpha$ and $\varepsilon$ amino groups of the lysine residue to mediate the thiol alkylation reaction. This linker peptide was synthesized by SPPS using Rink amide MBHA resin. The observed MALDI-TOF MS spectrum of the di-bromoacetyl linker is shown in figure $2.8 \mathrm{~B}$. In our studies of peptide dimerization, this di-bromoacetyl linker was used as a core entity for all V2 dimer derivatives prepared by thiol alkylation.

A

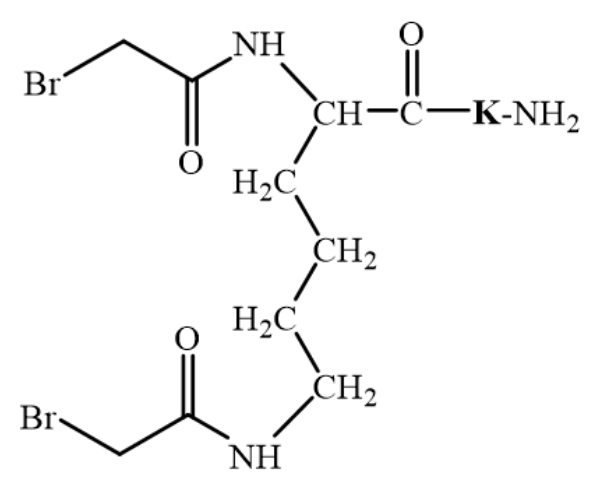

B

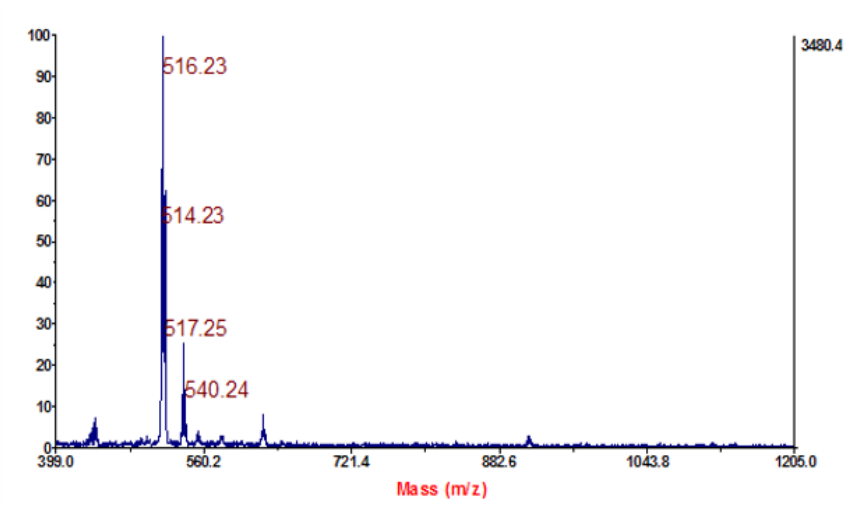

Figure 2.8 A. The di-bromoacetyl-KK-NH2 linker used in thiol alkylation. . B. MALDI-TOF MS of the linker. $\left[\mathrm{M}+\mathrm{H}^{+}\right]^{+} \mathrm{m} / \mathrm{z}=516.23$ (observed), 516.1 (calculated).

\subsubsection{V2-AGC dimerization}

Figure 2.9 shows the thiol alkylation-mediated dimerization of V2-AGC. Bromoacetyl linker is expressed in a compact form. For thiol-mediated dimerization of V2AGC, the peptide and the di-bromoacetyl linker were dissolved in a buffer consisting of $50 \%$ ACN in $\mathrm{H}_{2} \mathrm{O}(0.045 \%)$. The molar ratio of $\mathrm{V} 2-\mathrm{AGC} /$ bromoacetyl linker was 2.5/1. The $\mathrm{pH}$ of the reaction mixture was adjusted to 8 using solid $\mathrm{NaHCO}_{3}$. The reaction was monitored at 3, 6, 12 and $24 \mathrm{~h}$ using analytical HPLC. Once the reaction was complete, the desired dimer was purified by semi-preparative HPLC. There were side products formation probably due to free thiol and the reaction yield was approximately $75 \%$. The analytical HPLC monitoring of the dimerization reaction is shown in Figure 2.10, in which the reaction mixture at $\mathrm{t}=0 \mathrm{~h}$ and $24 \mathrm{~h}$ were analyzed. Figure 2.11 shows the analytical HPLC and MALDI-MS of purified V2-ACG dimer. 


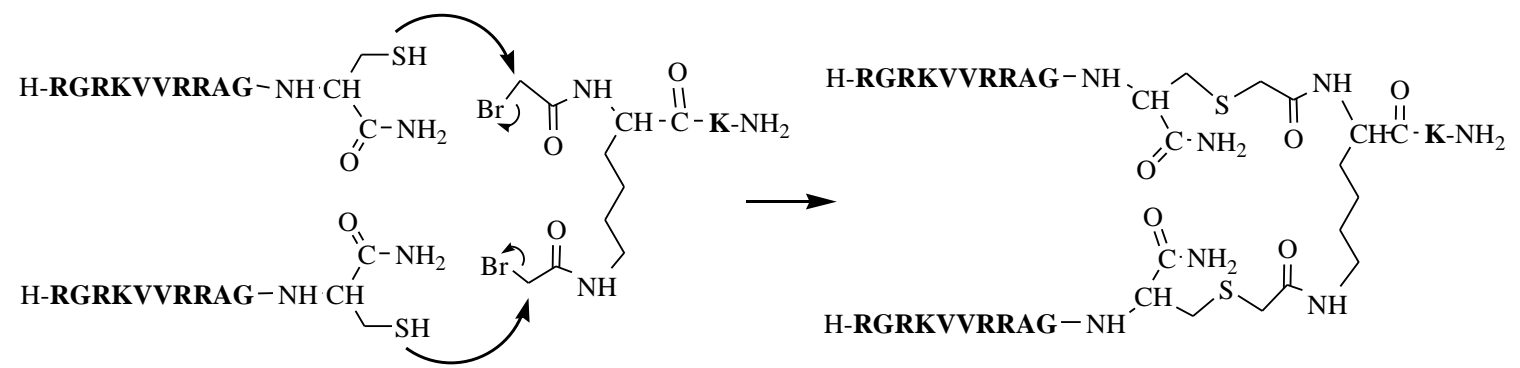

Figure 2.9 Reaction of V2-AGC with the di-bromoacetyl linker to form the V2-AGC dimer.
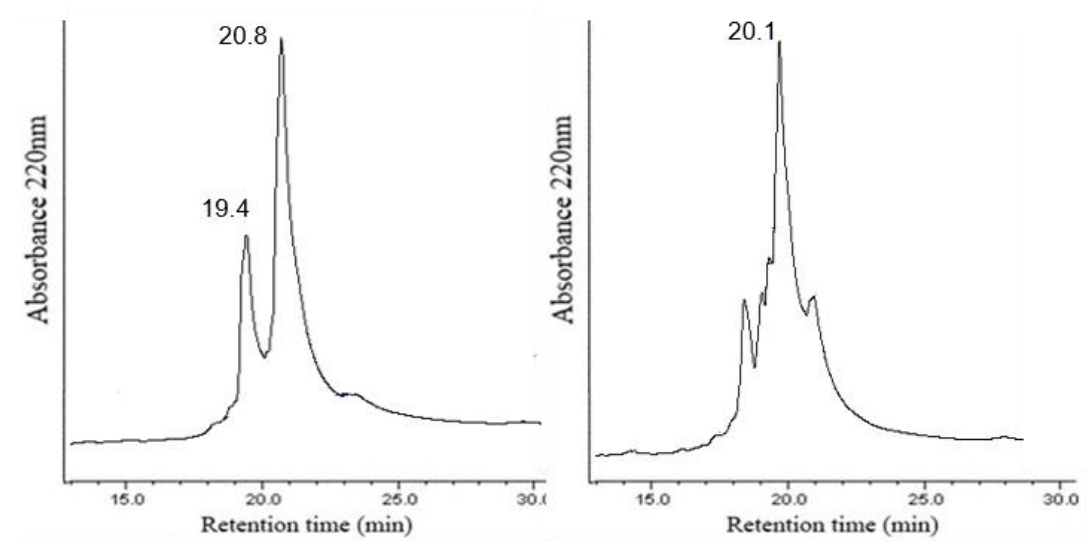

Figure 2.10. HPLC monitoring of V2-AGC dimerization reaction. Left panel: reaction mixture at time 0 . The peak at $19.4 \mathrm{~min}$ is the dibromo linker, and the peak at $20.8 \mathrm{~min}$ is V2-AGC. Right panel: reaction mixture at $24 \mathrm{~h}$. The peak at $20.1 \mathrm{~min}$ is the dimerized product as confirmed by MS analysis below.

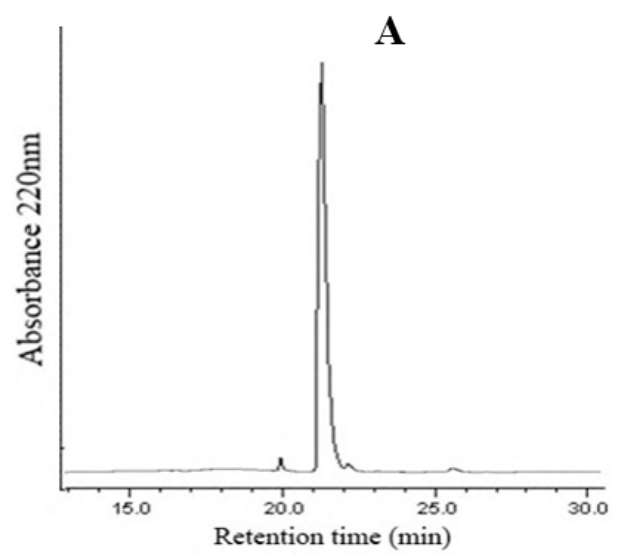

B

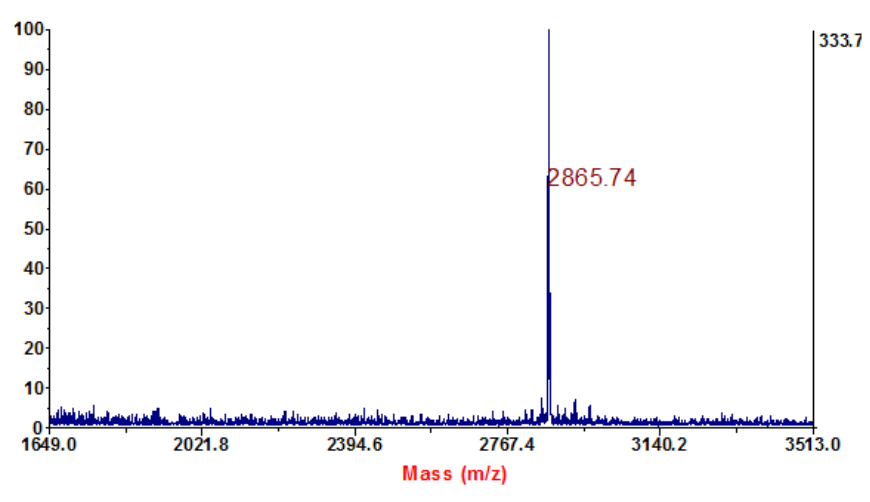

Figure 2.11 A. C18 analytical HPLC profile of purified V2-AGC dimer. Gradient: buffer B $0-40 \%$ in 40 mins. B. MALDI-TOF MS of purified peptide. $\left[\mathrm{M}+\mathrm{H}^{+}\right]^{+} \mathrm{m} / \mathrm{z}=2865.74$ (observed), 2866.48 (calculated). 


\subsubsection{V2-Cys dimerization}

The V2-Cys derivative has a cysteine at the C-terminus of the template sequence. Figure 2.12 shows the structure of V2-Cys. Dimerization of V2-Cys with the dibromoacetyl linker was conducted in the same way as for the previous V2-AGC dimerization. Figure 2.13 shows the HPLC profile of purified V2-Cys dimer peptide and its MALDI-TOF spectrum.
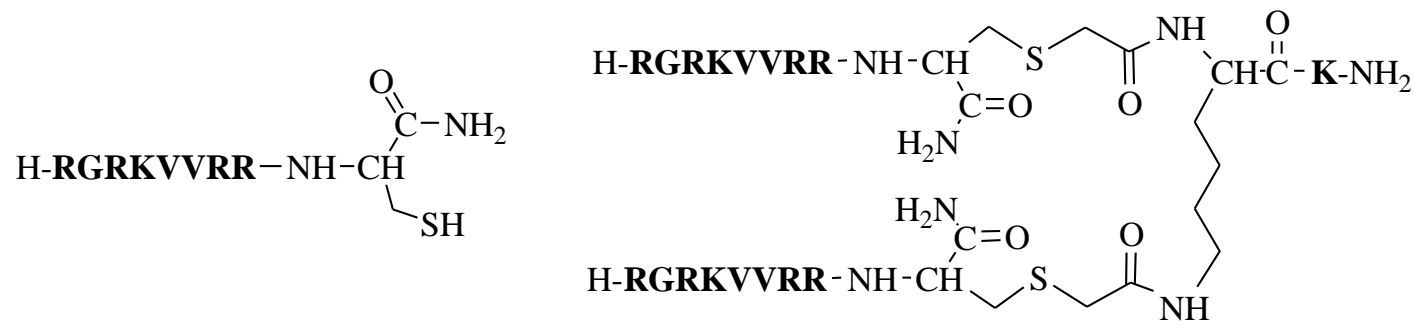

Figure 2.12 Left panel: the structure of V2-Cys. Right panel: V2-Cys dimer.

A

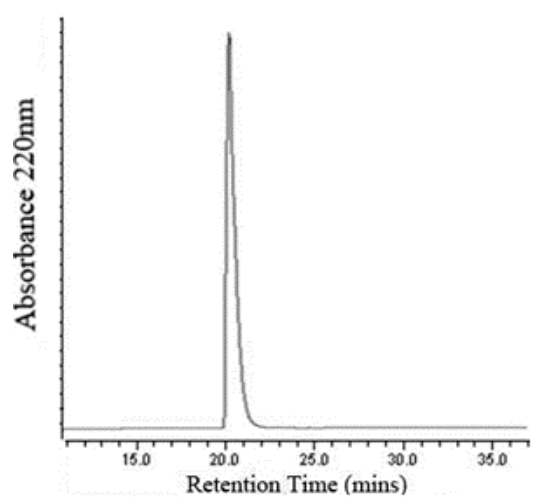

B

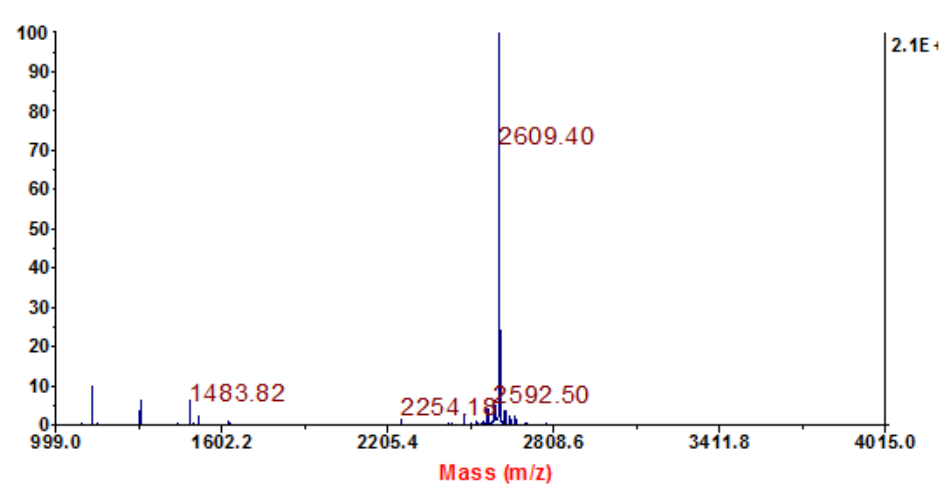

Figure 2.13 A. C18 analytical HPLC profile of pure V2-Cys dimer, Gradient buffer B 0-40\% in 40 mins. B. MALDI-TOF spectrum of HPLC fraction $\left[\mathrm{M}+\mathrm{H}^{+}\right]^{+} \mathrm{m} / \mathrm{z}=2609.40$ (observed), 2609.84 (calculated).

\subsubsection{V2-Cya dimerization}

V2-Cya has a cysteamine introduced at the C-terminus of the $\mathrm{V} 2$ template sequence. To synthesize V2-Cya, cysteamine $\left(\mathrm{HSCH}_{2} \mathrm{CH}_{2} \mathrm{NH}_{2}\right.$ ) was loaded onto a trityl resin through 
the thiol group and the V2 peptide sequence was assembled on the amino group of cysteamine using SPPS. Dimerization of V2-Cya was done with the di-bromoacetyl linker in a similar way. Figure 2.14 shows the structures of V2-Cya and V2-Cya dimer. Figure 2.15 shows the HPLC profile of purified V2-Cys dimer and its MALDI-TOF spectrum.

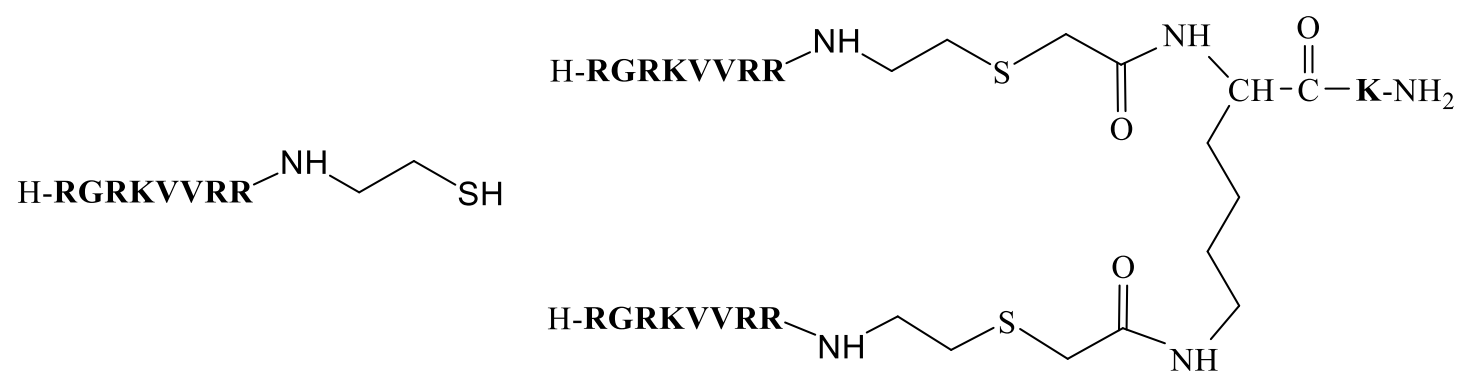

Figure 2.14 Structures of V2-Cya (left) and V2-Cys dimer (right).

A

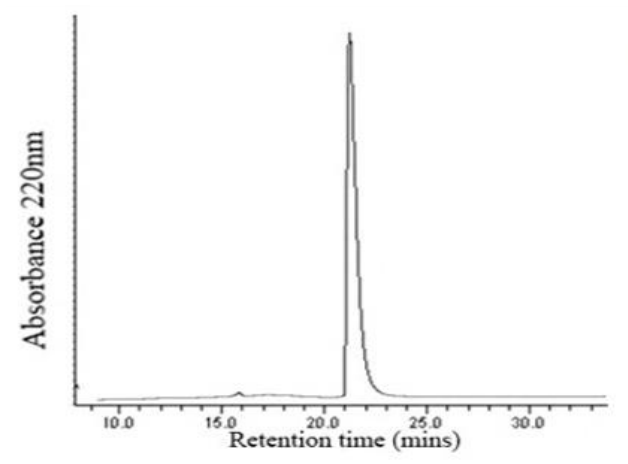

B

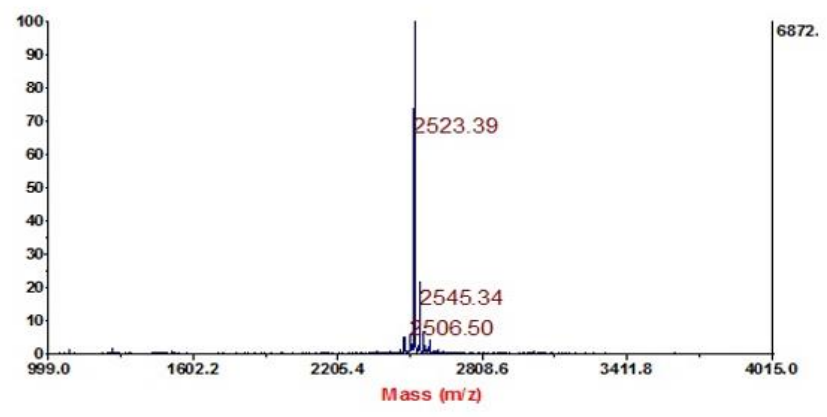

Figure 2.15 A. C18 analytical HPLC profile of purified V2-Cya dimer. Gradient: buffer B 0-40\% in 40 mins. B. MALDI-TOF spectrum of purified V2-Cya dimer. $\left[\mathrm{M}+\mathrm{H}^{+}\right]^{+} \mathrm{m} / \mathrm{z}=2523.39$ (observed), 2524.21(calculated). 


\subsubsection{Antibacterial activities of $\mathrm{V} 2$ peptide derivatives determined by radial diffusion assays}

After obtaining the purified V2 peptide derivatives, the two-step radial diffusion assays were conducted. Gram-positive and Gram-negative strains were used to determine MIC values of these V2 peptide variants. Various concentrations of the peptide variants were tested against E. coli, P. aeroginosa and $S$. aureus. MICs of the V2 peptide variants are shown in Table 2.3.

Table 2.3 MIC $(\mu \mathrm{M})$ of V2 peptides and the dimers against different bacterial strains.

\begin{tabular}{|c|c|c|c|c|}
\hline & & \multicolumn{2}{|c|}{ Gram negative } & \multirow{2}{*}{$\begin{array}{r}\text { Gram positive } \\
\text { S. aureus }\end{array}$} \\
\hline No & Peptide & E. coli & P. aeruginosa & \\
\hline 1 & V2 monomer & $39 \pm 3$ & $\sim 39$ & - \\
\hline 2 & V2-AGC & $18 \pm 1.5$ & $7 \pm 0.9$ & - \\
\hline 3 & V2 dimer & $1.9 \pm 0.3$ & $2.1 \pm 0.2$ & $2.6 \pm 0.3$ \\
\hline 4 & V2-Cya & - & - & - \\
\hline 5 & V2-Cys & - & - & - \\
\hline 6 & V2-AGC dimer & $2.1 \pm 0.3$ & $2.1 \pm 0.3$ & $1.9 \pm 0.2$ \\
\hline 7 & V2-Cys dimer & $1.1 \pm 0.2$ & $1.8 \pm 0.2$ & $1.2 \pm 0.2$ \\
\hline 8 & V2-Cya dimer & $1.6 \pm 0.3$ & $2.2 \pm 0.3$ & $1.8 \pm 0.2$ \\
\hline
\end{tabular}

V2 monomer 1 showed moderate activity against $E$. coli and P. aeroginosa with MIC of around $39 \mu \mathrm{M}$. However, V2-AGC 2, the monomeric V2 with three additional residues at the C-terminus, shows better antibacterial activity. It displays a MIC of $7 \mu \mathrm{M}$ against $P$. aeroginosa and $18 \mu \mathrm{M}$ against $E$. coli. It is possible that the cysteine residue in 
2 might form a disulfide linkage upon oxidation during the assay which leads to a dimer with lower MIC. All dimeric variants 6-8 formed by thiol alkylation reaction display excellent antibacterial activities. Considering the conformation of these dimeric variants, since their parent HBD-3 peptide exists in the form of $\beta$-sheet, they may maintain the same conformation when interacting with the bacterial membrane. We have not investigated the secondary structures of 6-8 in the lipid environment, and this is something that will need to be studied in the future. Studying the interaction with the bacterial membrane may provide useful information to explain variation in antibacterial activity of different peptides against different strains.

The V2 dimer 3 originally prepared by Liu et al. was reported to have good activity against Gram-positive and Gram-negative bacteria. ${ }^{59}$ Therefore, it was used as a control. Our data confirm that $\mathbf{3}$ possesses comparable activity against both Gram positive and Gram negative bacteria. The inner membranes of Gram-positive and Gram-negative bacteria are similar in compositions. In contrast, the major difference in their structure occurs at the outer membrane. Gram-positive bacteria consist of peptidoglycan in the outer membrane, whereas Gram-negative bacteria consist of LPS in the outer membrane. These differences in the inner and outer membranes considerably influence the membrane fluidity, charge state and amphiphilicity of the inner and outer membrane. Interestingly, the V2 peptide dimers are similarly active against Gram-positive and Gram-negative bacteria tested.

Table 2.3 shows that the thiol-alkylated dimers have comparable antibacterial activity as the control V2 dimer $\mathbf{3}$. In $\mathbf{6 - 8}$, the C-terminal moiety that is linked to the bivalent linker is different among the three dimers. This difference did not seem to have a significant impact on their antibacterial activities, although the V2-Cys dimer 7 is slightly more active than all other dimers.

As previously mentioned, the V2 peptide is derived from the C-terminal part of HBD-3, which is cationic. The V2 monomer 1 has a net charge of +6 , whereas the V2 
dimer 3 and the thiol-alkylated dimers 6-8 have a net charge of +13 . It reaffirms the results reported in the literature that, critical to the antibacterial mechanism, cationicity is essential for the electrostatic attraction of AMPs to the negatively charged phospholipid membrane of microbes. ${ }^{66}$ Cationicity is a vital feature of AMPs, and the charge state considerably influences their antibacterial property. ${ }^{33}$ It is believed that the electrostatic interactions lead AMP molecules to accumulate on the bacterial surface to a threshold level. ${ }^{33}$ Once the threshold is reached, hydrophobic interaction between nonpolar residues of the peptide and hydrophobic tails of the phospholipid molecules then enables peptide insertion into the membrane. ${ }^{66}$

The next stage that ultimately leads to the bacterial killing is somewhat controversial. There are three main models to explain AMPs' mode of action, namely "barrel-stave", "torroid pore" and "carpet" models. ${ }^{67}$ In the "barrel-stave" model, AMP monomers self-aggregate and form transmembrane pores, in which the hydrophobic patches of the AMP molecules interact with the acyl chains of phospholipid, whereas the hydrophilic surfaces form the pore lining. These channel-like pores lead to leakage of ions/metabolites, depolarization, membrane dysfunction and eventually to cell death. "Torroidal pore" model also describes the formation of the transmembrane pore, but the primary difference between this model and the "barrel-stave" model is that lipids are intercalated with peptide in the transmembrane channel, which is lined with both polar peptide facets and phospholipid head groups. The "carpet" model proposes a completely different mechanism from the previous models. First, a high density of peptides accumulates on the membrane surface, carpeting the phospholipid bilayer. When a critical concentration is reached, the membrane is disrupted due to unfavorable energetics. Based on the high cationicity of V2 monomeric and dimeric variants, it is implied that these peptides probably follow carpet or torroidal model. However, this is not established and further biophysical studies are necessary to provide evidence to support this hypothesis. 


\subsubsection{Ab-initio designed DFTamP1 and its dimers}

Wang et $a .^{40}$ designed a peptide named DFTamP1 and its derivatives using the Abinitio approach ${ }^{40}$. DFTamP1-p was designed to target methicillin-resistant Staphylococcus aureus. The peptide was reported to cause bacterial surface damage and kill communityassociated MRSA (methicillin resistant Staphylococcus aureus). Unlike most of the AMPs, this potent anti-MRSA peptide was less cationic and more hydrophobic in nature. In our study, the biologically active derivative of the DFTamP1 peptide was used as the template to synthesize different variants listed in Table 2.4. The DFTamP1-p was synthesized and used as a control. The sequence of DFTamP1-p is H-GLLPLLSLLGKLL-NH ${ }_{2}$. It was synthesized by SPPS using rink amide MBHA resin. Figure 2.16 shows the HPLC profile and MALDI-TOF MS of the purified peptide.
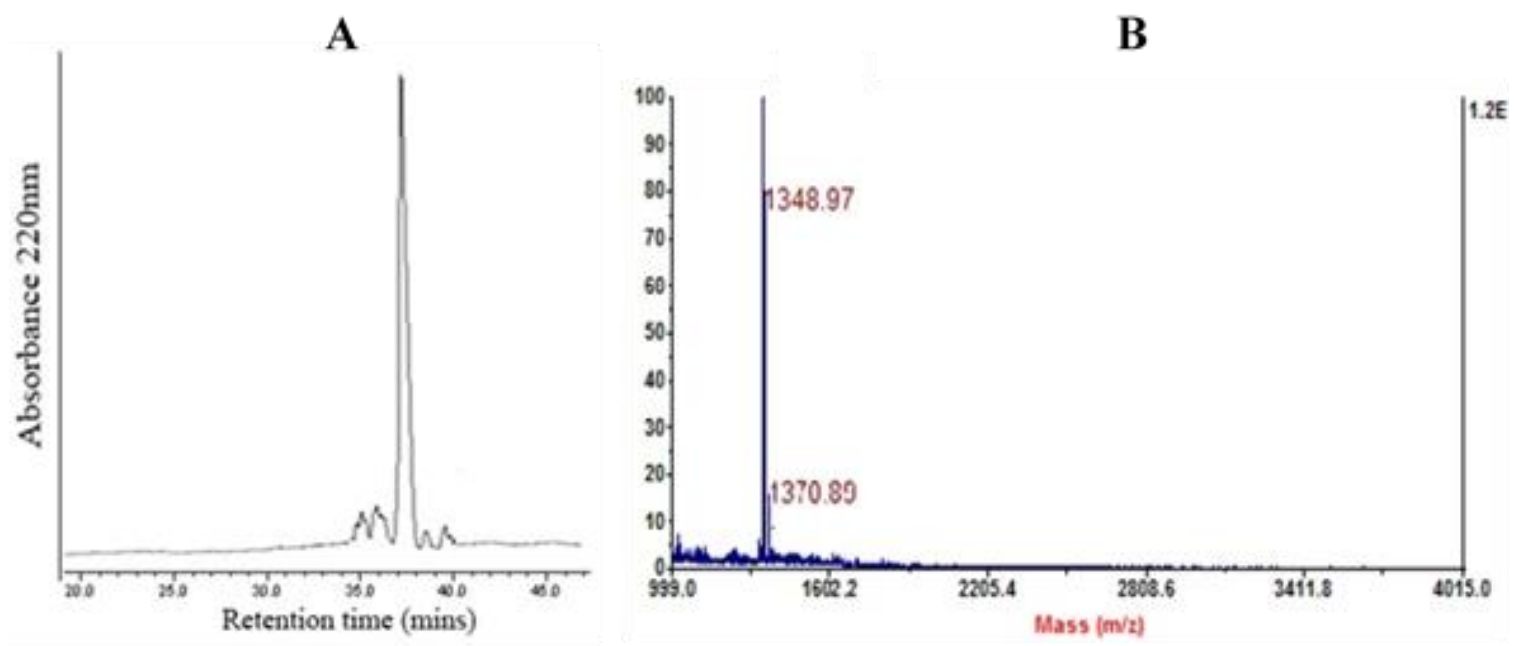

Figure 2.16 A. C18 analytical HPLC profile of DFTamP1-p. Gradient: Buffer B 0-100\% in 50 mins. B MALDI-TOF MS of the purified peptide. $\left[\mathrm{M}+\mathrm{H}^{+}\right]^{+} \mathrm{m} / \mathrm{z}=1348.97$ (observed), 1348.78 (calculated).

Different monomeric and dimeric variants of this peptide were synthesized as shown in Table 2.4. Similarly, thiol alkylation reactions were conducted with DFTamP1-p variants to synthesize DFTamP1-p dimers. 
Table 2.4 List of all DFTamP1-p related peptides synthesized in this study.

\begin{tabular}{|c|c|c|}
\hline No & Sequence of peptide & Name of peptide \\
\hline 9 & $\mathrm{Br}-\mathrm{CH}_{2} \mathrm{CO}-\left(\mathrm{Br}-\mathrm{CH}_{2} \mathrm{CO}-\right) \mathbf{K K}-\mathrm{NH}_{2}$ & Bromoacetyl linker \\
\hline 10 & H-GLLPLLSLLGKLL-NH ${ }_{2} *$ & DFTamP1-p \\
\hline 11 & H-GLLPLLSLLGKLLC-NH ${ }_{2}$ & DFTamP1-Cys \\
\hline 12 & H- GLLPLLSLLGKLL-NH- $\mathrm{CH}_{2}-\mathrm{CH}_{2}-\mathrm{SH}$ & DFTamP1-Cya \\
\hline 13 & (H-GLLPLLSLLGKLL) ${ }_{2} \mathbf{K K}-\mathrm{NH}_{2}$ & DFTamP1- dimer \\
\hline 14 & H-GLLPLLSLLGKLLGPNGLLPLLSLLGKLL-NH ${ }_{2}$ & GPN-dimer \\
\hline 15 & (H-GLLPLLSLLGKLLC- $\left.\mathrm{CH}_{2} \mathrm{CO}\right)_{2} \mathbf{K K}-\mathrm{NH}_{2}$ & $\begin{array}{l}\text { DFTamP1-Cys } \\
\text { dimer }\end{array}$ \\
\hline
\end{tabular}

Letters in bold denote amino acids residues. The red color part denotes thiol-containing moieties. *DFTamP1-p as a control.

\subsubsection{DFTamP1-Cys}

DFTamP1-Cys 11 contains a cysteine at its C-terminus. Figure 2.17 shows the HPLC profile and MALDI-TOF MS of the purified peptide. Its MALDI-TOF spectrum also shows some isotopic forms and the sodium adduct (1476 Da).
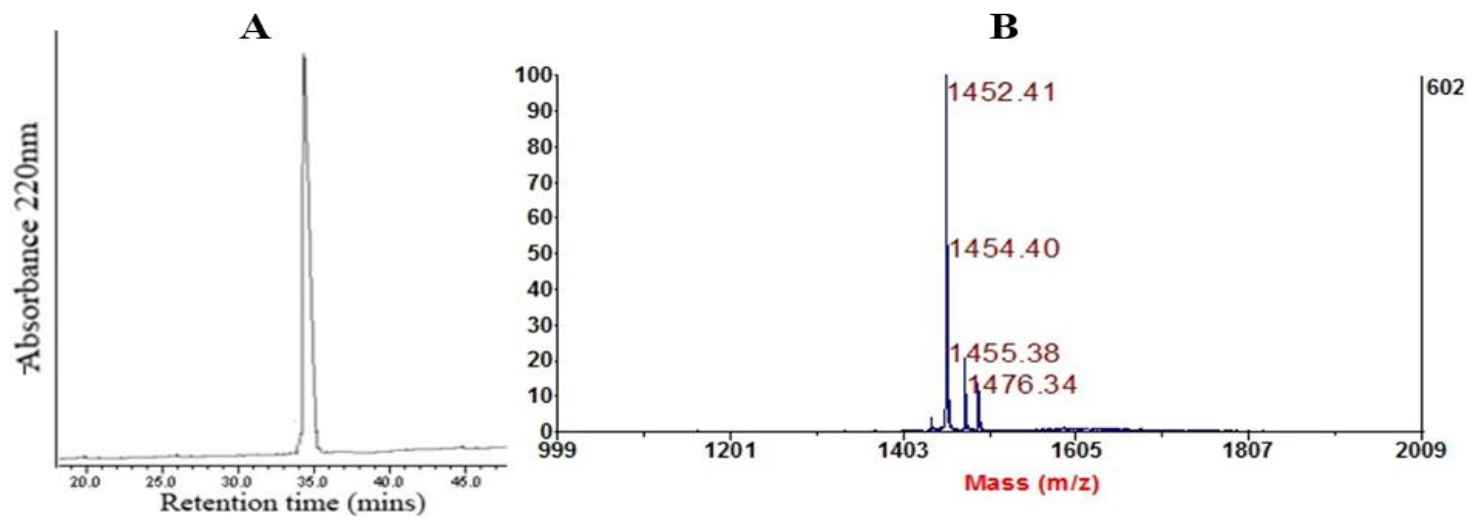

Figure 2.17 A. C18 analytical HPLC profile of pure DFTamP1-Cys. Gradient: buffer B 0-100\% in $50 \mathrm{~min}$. B. MALDI-TOF of purified peptide. $\left[\mathrm{M}+\mathrm{H}^{+}\right]^{+} \mathrm{m} / \mathrm{z}=1452.41$ (observed), 1451.93(calculated). 


\subsubsection{DFTamP1-Cya}

The cysteamine-containing derivative of DFTamP1-p was synthesized as described in the section 2.4.3. Figure 2.18 shows its structure, and it was termed as DFTamP-Cya 12. Figure 2.19 shows the HPLC profile and MALDI-TOF MS of the purified peptide.

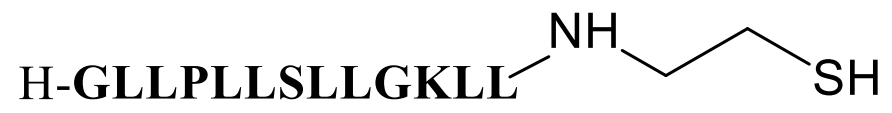

Figure 2.18 Structure of DFTamP1-Cya 12.
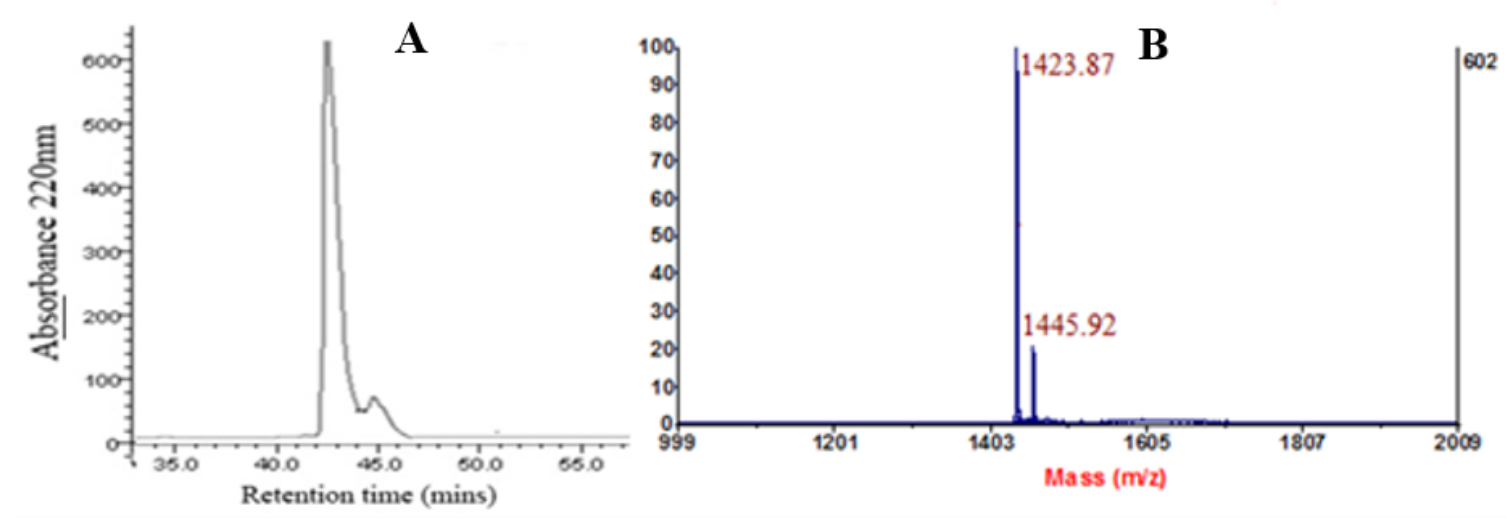

Figure 2.19 A. C18 analytical HPLC profile of pure DFTamP1-Cya. Gradient: buffer B 0-100\% in 50 mins. B MALDI-TOF MS of purified DFTamP1-Cya. $\left[\mathrm{M}+\mathrm{H}^{+}\right]^{+} \mathrm{m} / \mathrm{z}=1423.87$ (observed), 1423.93(calculated).

\subsubsection{Dimerization of DFTamP1-Cys with di-bromoacetyl linker}

Thiol alkylation of the more hydrophobic and less cationic DFTamP1-p derivative DFTamP1-Cys 11 was conducted with the di-bromoacetyl linker 9. Like previous dimerization reactions, the ratio of DFTamP1-Cys $\mathbf{1 1}$ to the di-bromoacetyl linker $\mathbf{9}$ was $2.5 / 1$, and the reaction was conducted at an alkaline $\mathrm{pH}$ of 8 . The reaction was completed in $24 \mathrm{~h}$. Semi-preparative HPLC was used to purify the desired peptide from the reaction mixture. Figure 2.20 shows the structure of the dimer formed, and it was termed as DFTamP1-Cys dimer 15. Figure 2.21 shows the HPLC profile and MALDI-TOF MS of the purified peptide. 


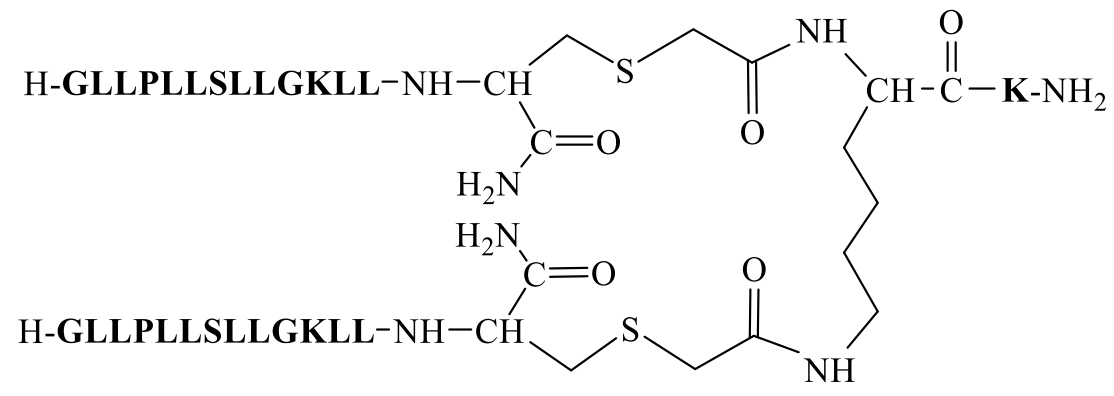

Figure 2.20 Structure of DFTamP1-Cys dimer 15 formed by the thiol-alkylation reaction.
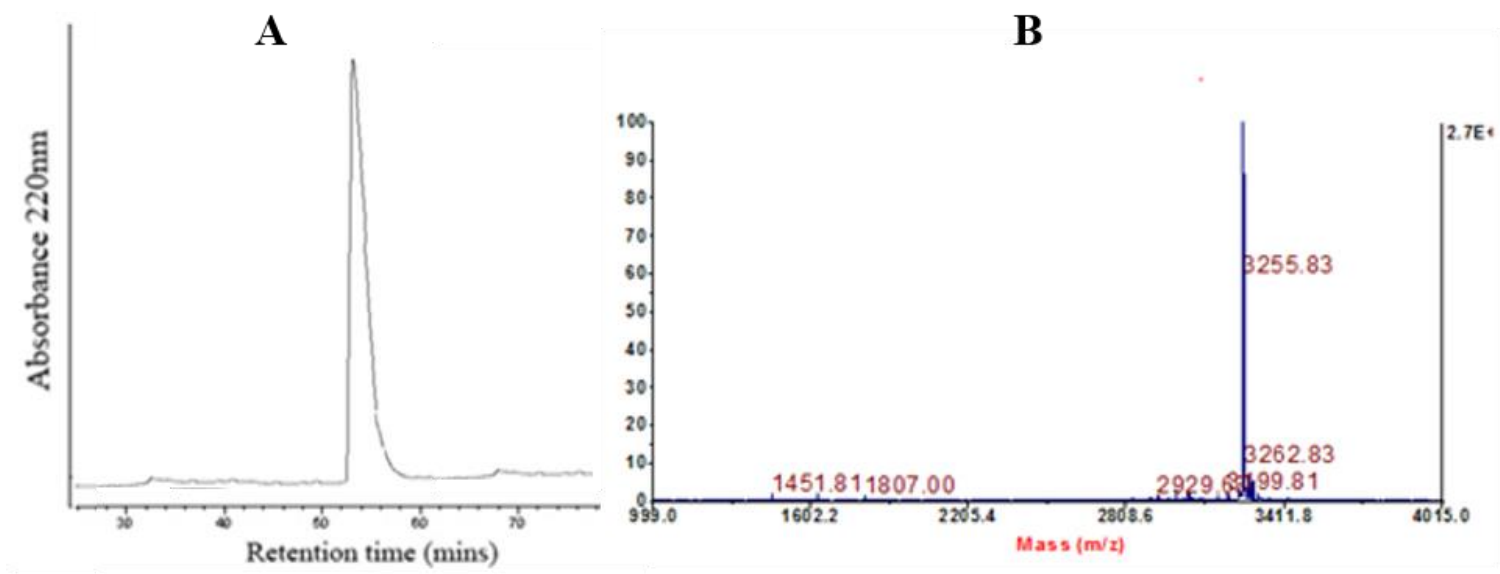

Figure 2.21 A. C18 analytical HPLC profile of pure DFTamP1-Cys dimer. Gradient: buffer B 0-100\% in 50 mins. B. MALDI-TOF MS of the purified peptide. $\left[\mathrm{M}+\mathrm{H}^{+}\right]^{+} \mathrm{m} / \mathrm{z}=3255.83$ (observed), 3257.24 (calculated).

\subsubsection{The GPN Dimer}

A linear sequential dimer was synthesized using the template sequence derived from DFTamP-p. The peptide is named as GPN-dimer, as the two DFTamP-p units are joined together through a tripeptide GPN spacer. The full sequence of the GPN dimer is GLLPLLSLLGKLLGPNGLLPLLSLLGKLL. The GPN motif has a great propensity to form a turn, forcing the peptide to form a hairpin structure (Figure 2.22). It is hypothesized that the two DFTamP1-p sequences would be arranged in an antiparallel way in the GPNlinked dimer. This peptide was synthesized by SPPS directly using Rink amide MBHA resin. Figure 2.23 shows the HPLC profile and MALDI-TOF MS of the purified peptide. 
The MALDI-TOF spectrum of MS analysis reveals the presence of the sodium adduct: $[\mathrm{M}+\mathrm{H}]^{+}=2971.08$.

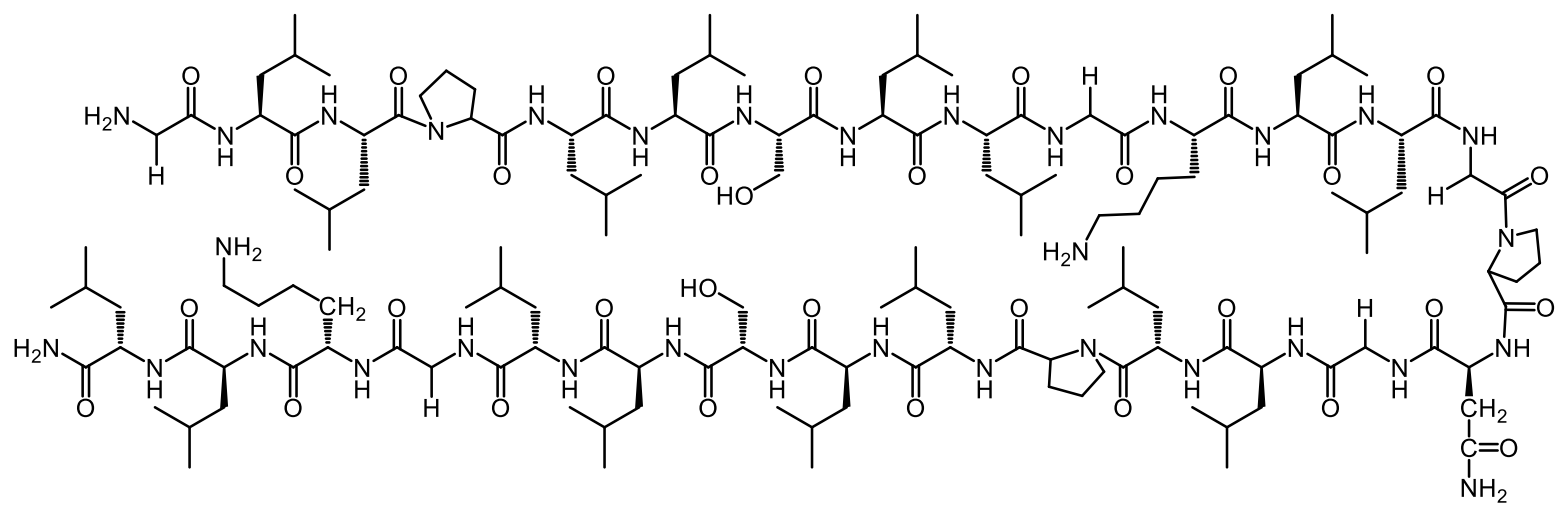

Figure 2.22 GPN linked dimer 14 .
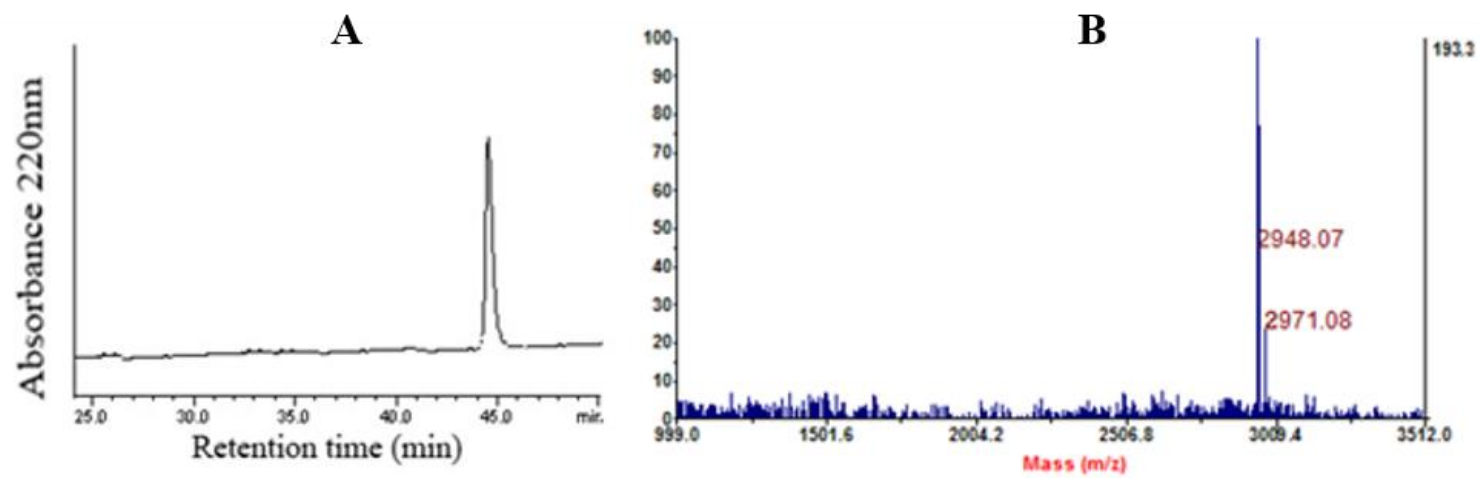

Figure 2.23 A. C18 analytical HPLC profile of purified GPN dimer 14. Gradient: buffer:B 50-100\% 50 mins. B. MALDI-TOF spectrum of purified GPN-dimer. $\left[\mathrm{M}+\mathrm{H}^{+}\right]^{+} \mathrm{m} / \mathrm{z}=2948.07$ (observed), 2948.81(calculated).

\subsubsection{The parallel DFTamP1-p dimer}

This parallel dimer of DFTamP1-p was synthesized by SPPS on the bivalent Lys using protocol of James Tam. ${ }^{42}$ Unlike the V2 peptide, DFTamP1-p is less cationic and more hydrophobic. DFTamp1-p is reported to adopt a $\alpha$-helical structure where the hydrophilic and hydrophobic side chains were clustered on opposite faces. We anticipated that the two monomer chains in the parallel DFTamP1-dimer would also adopt a helical 
conformation. Figure 2.24 shows the structure of DFTamP1-dimer synthesized by SPPS using Rink amide MBHA resin. Figure 2.25 shows the HPLC profile and MALDI-TOF MS of purified DFTamP1-dimer.

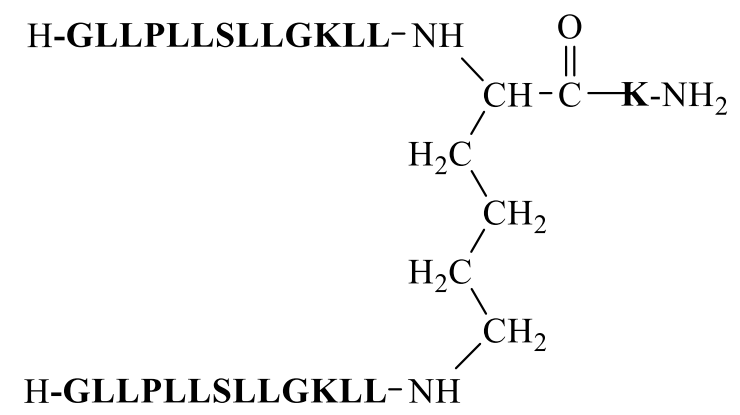

Figure 2.24 Structure of the parallel DFTamP1-dimer 13 .
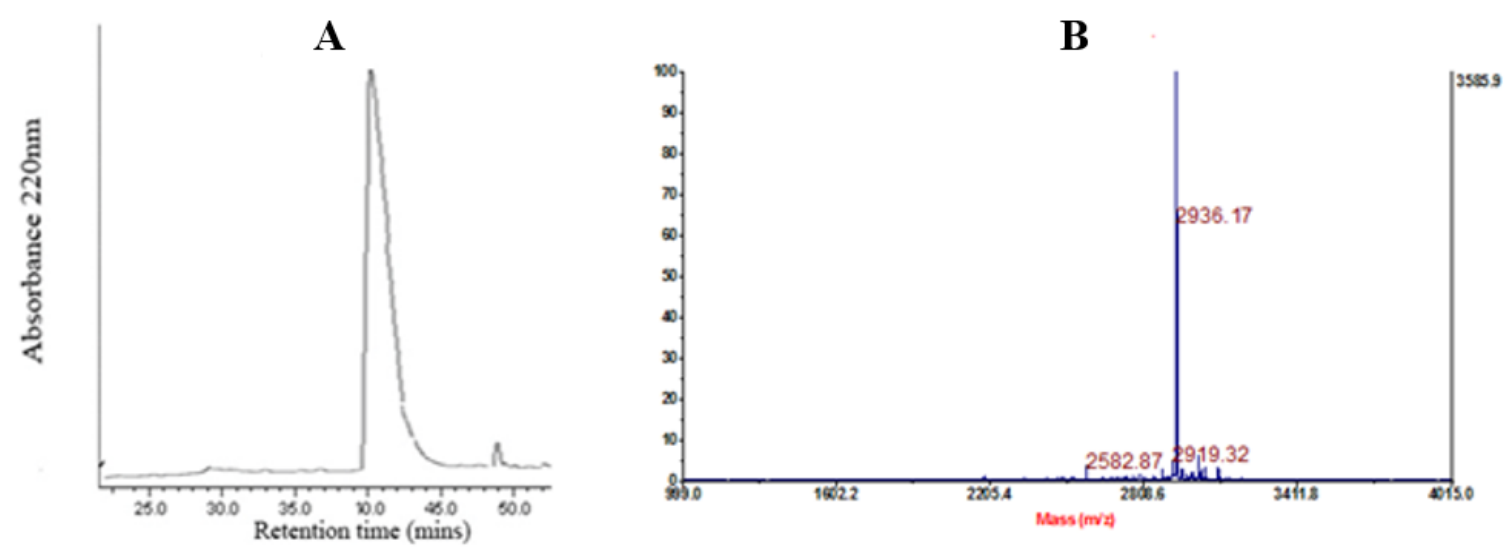

Figure 2.25 A. C18 analytical HPLC profile of DFTamP1-dimer (13). Gradient: buffer B 0-100\% in 50 mins. B. MALDI-TOF MS of purified DFTamP1-dimer. $\left[\mathrm{M}+\mathrm{H}^{+}\right]^{+} \mathrm{m} / \mathrm{z}=2936.17$ (observed), 2935.91(calculated).

\subsubsection{Radial diffusion assays of DFTamP1-p and its variants}

To determine the antibacterial activities of different DFtamP1-p variants synthesized, the same two-step radial diffusion assay was conducted. The assays were conducted against both gram-positive and gram-negative bacteria. Two gram-positive, i.e. Staphylococcus aureus and Bacillus subtilis along with two-gram negative, i.e., Pseudomonas aeroginosa and Escherichia coli, were used. The MICs of different 
DFTamP1-p derivatives obtained from the graphs of the diameter of the zone of inhibition against $\log _{10}$ of concentration are shown in Table 2. 6. DFTamP1-p is a positive control in this study.

Table 2.5 MICs $(\mu \mathrm{M})$ of DFTamp1-p variants.

\begin{tabular}{|c|c|c|c|c|}
\hline \multirow[b]{2}{*}{ No Peptide } & \multicolumn{2}{|c|}{ Gram negative bacteria } & \multicolumn{2}{|c|}{ Gram positive bacteria } \\
\hline & E. coli & P. aeruginosa & S. aureus & B. subtilis \\
\hline 10 DFTamP1-p & $6.3 \pm 0.5$ & $3.9 \pm 0.3$ & $3.5 \pm 0.3$ & $8.1 \pm 0.9$ \\
\hline 11 DFTamP1-Cys & $1.3 \pm 0.2$ & $4.4 \pm 0.5$ & $2.5 \pm 0.2$ & $3.3 \pm 0.3$ \\
\hline 12 DFTamP1-Cya & $4.4 \pm 0.5$ & $6.5 \pm 0.8$ & $4.1 \pm 0.4$ & $5.7 \pm 0.6$ \\
\hline 13 DFTamP1-p dimer & $8.3 \pm 1$ & $59.9 \pm 13$ & $22.3 \pm 3$ & $16.9 \pm 2$ \\
\hline 14 GPN- linked dimer & $6.1 \pm 0.7$ & $105.7 \pm 27$ & $8.7 \pm 1$ & $109.4 \pm 30$ \\
\hline 15 DFTamP1-Cys dimer & $10.6 \pm 1$ & $29.4 \pm 3$ & $11.9 \pm 1.5$ & $19.2 \pm 3$ \\
\hline \multicolumn{5}{|c|}{$\begin{array}{l}\text { The MICs were calculated using } 2 \text {-step radial diffusion assays for all peptides and using dilutions of } 1000 \mu \mathrm{M}, 500 \mu \mathrm{M} \text {, } \\
100 \mu \mathrm{M}, 50 \mu \mathrm{M}, 25 \mu \mathrm{M}, 10 \mu \mathrm{M} \text { and } 5 \mu \mathrm{M} \text { against Gram-positive and Gram-negative bacteria. Each peptide was prepared } \\
\text { in a stock solution } 70 \% \mathrm{ACN}: 30 \% \mathrm{ddH}_{2} \mathrm{O} \text {, diluted to required concentration with } \mathrm{ddH}_{2} \mathrm{O} \text {. All dimers were completely } \\
\text { solubilized. }\end{array}$} \\
\hline
\end{tabular}

The antibacterial testing of all peptides was performed with the samples dissolved in $\mathrm{ddH}_{2} \mathrm{O}$. However, 13 and $\mathbf{1 4}$ were not readily soluble in $\mathrm{ddH}_{2} \mathrm{O}$. Hence, $10 \% \mathrm{ACN}$ in $\mathrm{H}_{2} \mathrm{O}$ was used to facilitate the dissolution of the two dimers. From Table 2.6, the antibacterial activity of the three dimers $\mathbf{1 3 - 1 5}$ is noticeably poorer than the control monomer 10.

Of all the peptides made, only $\mathbf{1 1}$ was observed to have a marginally lower MIC than $\mathbf{1 0}$ on E. coli, S. aureus and B. subtilis (Table 2.6), suggesting that addition of a Cys residue at the C-terminus slightly increased the antibacterial activity of the peptide. The addition 
of cysteine might have contributed to consolidating the amphiphilic nature of its helical conformation. A more rigid $\alpha$ helical conformation may aid the peptide in penetrating into the bacterial cell membrane. The MICs obtained for the C-ter cysteamine-containing monomer $\mathbf{1 2}$ showed that it had antibacterial activity comparable to that of $\mathbf{1 0}$ (Table 2.5).

The results of $\mathbf{1 0}$ confirm previous findings that DFTamP1 is active against both gram-positive and gram-negative bacteria. ${ }^{40}$ DFTamP1 adopts a helical structure where hydrophobic and hydrophilic side chains are clustered on opposite sides. In the helical conformation, the leucine residues play a significant role in membrane binding as they constitute the hydrophobic side of the helix. It was proposed that the broad hydrophobic surface of $\mathbf{1 0}$ is crucial for its potency against bacteria allowing the peptide nonselectively attach with the bacterial membrane of both gram-positive and gramnegatives species. According to the membrane perturbation model, the broad surface of DFTamP1 facilitates the peptide to infiltrate deeper into bacterial membranes and excise its damaging effect. ${ }^{40}$ Other residues are important in maintaining the overall amphiphilic conformation.

Interestingly, the two parallel dimers 13 and 15 and the sequential GPN-dimer 14 show lower antibacterial activity than the monomeric parent peptide 10. So for a highly hydrophobic and less cationic peptide like DFTamP1, dimerization is counterproductive for the antibacterial efficacy. The initial study of ab-initio model proposes that the broad hydrophobic surface of $\mathbf{1 0}$ facilitates the peptide to infiltrate deeper into the bacterial membrane. ${ }^{40}$ Probably, dimerization has affected the ability of the peptide to infiltrate into the membrane. A possible reason is that the hydrophobic surfaces of the two peptide chains self-associate in the dimer, making them unavailable for interacting with the bacterial cell membrane. In a study by Yuxin et al., ${ }^{68}$ it was reported that a higher ability of the peptide to self-associate in solution can be correlated with weaker antimicrobial activity. Self-association between the peptide chains in DFTamP1 dimers may be 
responsible for the poor MICs observed in our study, as this would lead to slower or reduced accumulation of peptide molecules on the bacterial surface and therefore poor killing activity.

\subsection{Conclusion}

Unrestricted use of antibiotics has caused the problem of resistant strains which has emerged as a serious threat to public health. This necessitates the search for alternatives to existing antibiotics. AMPs display huge potential to become a new generation of antibacterial agents. Dimerization is an effective way to make certain AMPs more potent. SPPS is commonly used to synthesize peptide dimers. However, this approach has limitations in terms of efficiency, yield and peptide chain aggregation. We used a different and effective method to dimerize peptides by thiol alkylation. The method is simple, and it addresses the limitations of SPPS in preparing long peptides. Thiol alkylation is also useful in conjugating and dimerizing peptides. The $\mathrm{V} 2$ dimer derivatives synthesized by this technique have improved antibacterial activity compared to the monomer. However, the antibacterial activity of the less cationic but more hydrophobic DFTamP1-p decreases after dimerization whether the peptide is dimerized by thiol alkylation or SPPS. The addition of a cysteine residue at the C-terminus of DFTamP1-p slightly enhanced antibacterial activity of the peptide. Hence, the low cationic and more hydrophobic peptides like DFTamP1-p function better as a monomer. This study is a small step toward understanding the key features of AMPs and their design principles. More studies are needed to help understand other parameters of AMPs such as selectivity index, hemotoxicity, secondary structure and sequence to develop more potent AMPs that are therapeutically useful. 


\subsection{Materials and Methods}

\subsubsection{Chemicals}

Benzotriazol-1-yl-oxytripyrrolidinophosphonium hexafluorophosphate (PyBOP), Rink Amide-MBHA resin, 2-chlorotrityl chloride resin, Fmoc-Ser(tBu)-OH, and hydroxybenzotriazole (HOBT) were obtained from GL Biochem (Shanghai, China). FmocArg(Pbf)-OH, Fmoc-Val-OH, Fmoc-Lys(Boc)-OH, Fmoc-Lys(Fmoc)-OH, Fmoc-Pro-OH, Fmoc-Cys(Trt)-OH, Fmoc-Asn(Trt)-OH, and Fmoc-Lys(Boc)-OH were purchased from Novabiochem. Fmoc-Lys (Fmoc)-OH was purchased from Chem-Impex. $N, \quad N$,Diisopropylethylamine (DIEA) was obtained from Applied Biosystems. $N$, $N$,Dimethylformamide (DMF), dichloromethane (DCM), diethyl ether, and HPLC-grade acetonitrile were purchased from Fisher Scientific. Bromoacetic acid, trifluoroacetic acid (TFA) and triisopropylsilane (TIS) were purchased from Alfa aesar. Ethanedithiol was obtained from Fluka Chemical. Piperidine was obtained from Acrons. All other reagents were purchased from Sigma-Aldrich.

\subsubsection{Media and Reagents}

1. Trypticase soy broth (TSB, Difco, Detroit, MI). Full-strength broth contains 30 $\mathrm{g}$ of powdered medium per liter of deionized water. It is autoclaved for $20 \mathrm{~min}$ at $121^{\circ} \mathrm{C}$ and stored at room temperature.

2. Phosphate buffer Stock solutions. $100 \mathrm{mM}$ of phosphate buffer solution (pH 7.2) was prepared using standard monobasic sodium phosphate $\left(\mathrm{NaH}_{2} \mathrm{PO}_{4} .2 \mathrm{H}_{2} \mathrm{O}\right.$, Fisher) and dibasic sodium phosphate $\left(\mathrm{Na}_{2} \mathrm{HPO}_{4} .7 \mathrm{H}_{2} \mathrm{O}\right.$ Fisher) solutions. The resulting buffer is autoclaved at $121^{\circ} \mathrm{C}$ for $20 \mathrm{~min}$ and then stored at room temperature.

3. Agarose (Sigma). Using low electroendosmosis (EEO)-type agarose is critical to limit electrostatic interactions between positively charged antimicrobial peptides and sulfated moieties of standard agar. 
4. Underlay gels. Mix $50 \mathrm{~mL}$ of $100 \mathrm{mM}$ sodium phosphate buffer with $5 \mathrm{~mL}$ fullstrength trypticase soy broth in a 1000-mL Pyrex beaker, add 5 g agarose (Sigma) and bring the volume to $500 \mathrm{~mL}$ with deionized distilled water. Adjust the final $\mathrm{pH}$ to 7.4 or 6.5 with $1 \mathrm{~N} \mathrm{NaOH}$ or $\mathrm{HCl}$. Dissolve agarose and dispense required quantity in a separate vessel, autoclave at $121{ }^{\circ} \mathrm{C}$ for $20 \mathrm{~min}$, and store the sterilized media at room temperature. Before use, the solidified medium should be fluidized (use a microwave oven) and placed into a water bath maintained at $42^{\circ} \mathrm{C}$.

5. Overlay agar. This agar contains $60 \mathrm{~g}$ (twice the customary amount) of trypticase soy broth (TSB, Difco) and $10 \mathrm{~g}$ of agarose (Sigma) per liter of deionized water. Dissolve the suspension and dispense the required quantity in a separate appropriate-sized vessel, then autoclave it for $20 \mathrm{~min}$ at $121^{\circ} \mathrm{C}$. The sterilized overlay agar is stored at room temperature and before use, it is heated in a microwave oven to re-dissolve the agarose, placed in a $42^{\circ} \mathrm{C}$ water bath to keep it molten.

6. $10 \mathrm{mM}$ sodium phosphate buffer, $\mathbf{p H}$ 7.4. It was prepared by diluting $100 \mathrm{mM}$ sodium phosphate buffer, sterilized and maintained at room temperature. It was chilled before use to wash bacteria.

\subsubsection{Solid Phase Peptide Synthesis}

We used Fmoc protected amino acids and compatible resins for SPPS. For Fmoc protected Rink resin, Fmoc was first removed using 20\% piperidine (DMF). Usually in first round resin was washed for $5 \mathrm{~min}$ then for another $20 \mathrm{~min}$. After that, the resin was washed with DMF (2x) DCM (2x), DMF $(2 \times) .{ }^{69}$ To couple the first C-ter amino acid, Fmoc protected amino acid (4 eq.) and PyBOP (4 eq.) were dissolved in DMF, then DIEA (8 eq.) was added to the solution. The solution was added into a reaction vessel containing the resin. It takes about one hour for most of the amino acids and around two and half hours for a few other amino acids (e.g., arginine) to couple. The coupling efficiency was monitored by Kaiser test. ${ }^{70}$ For recoupling, the same amino acid and the same or a stronger 
activation agent such as HATU were dissolved in DMF. DIEA (8 eq.) was added to the solution, then the solution was added into the resin. Once the coupling was complete, the resin was washed with DMF (2x), DCM (2x), DMF (2x). At times when the resin was not completely coupled, it was necessary to cap the uncoupled amine by acetic anhydride. For peptide chain elongation, Fmoc deprotection and couplings of the following amino acids were performed by using the same procedures as described above. The whole peptide sequence to be synthesized will assemble on this resin. Therefore it is inevitable to ensure that resin is completely protected.

Peptides synthesized are listed in Table 2.6. Most of the peptides were synthesized using Rink amide MBHA resin except those with a C-terminal amide formed with cysteamine. The substitution of Rink Amide MBHA is $0.65 \mathrm{mmol} / \mathrm{g}$. Cysteamine was loaded using 2-chloro trityl chloride resin. Initially, the resin was swollen using 30\% TFA; it immediately changed its color to red as a tertiary carbocation was formed. After removing TFA, 4 molar equivalents of cysteamine in DCMIDMF solution was added to the swollen resin, and the red color of resin disappeared almost immediately, indicating the cation was quenched by the thiol group of cysteamine. After shaking for some time, the whole resin color changed to yellowish white, and it was assumed that cysteamine was loaded to the resin. The resin was then washed with $\operatorname{DCM}(2 x), \operatorname{DMF}(2 x), \operatorname{DCM}(2 x)$. Ninhydrin (Kaiser) test was conducted to verify cysteamine coupling. Then peptide synthesis was conducted using the standard Fmoc SPPS protocols. Peptides having a C-ter amide formed with cysteamine and synthesized using 2-chlorotrityl chloride resin are:

- H-RGRKVVRR-NH-CH $2-\mathrm{CH}_{2}-\mathrm{SH}$

- H-GLLPLLSLLGKLL-NH- $\mathrm{CH}_{2}-\mathrm{CH}_{2}-\mathrm{SH}$

After completing the sequence, the peptides were cleaved from the resin using a cocktail containing (TFA 95\%, $\mathrm{H}_{2} \mathrm{O} 2.5 \%$, TIS $2.5 \%$ ). However, for the peptides containing cysteine or cysteamine, the composition of the cocktail solution was (TFA $92.5 \%, \mathrm{H}_{2} \mathrm{O} 2.5 \%$, TIS $2.5 \%$, ethanedithiol 2.5\%). The final cleavage reaction was done for 3 hours for peptides containing arginine or 1 to 1.5 hours for other peptides. The longer reaction time for Arg-containing peptides was to ensure the arylsulfonyl(Pbf) protecting 
group on Arg was removed. After cleavage, the resin was filtered out and solute was precipitated with chilled ether. Precipitated peptides were analyzed by analytical C18 HPLC and purified with C18 preparative HPLC. ESI-MS and MALDI were used to identify the peptides collected in the HPLC fractions. MALDI-MS results are shown for most peptides as the molecular weight of the peptides studied in this project are all less than $4000 \mathrm{Da}$ and so can be measured with sufficient accuracy by MALDI MS. ESI-MS was also used for some peptides. Pure peptide fractions were pooled, lyophilized and stored at $-20^{\circ} \mathrm{C}$. The lyophilized power give the final product as TFA salt.

Table 2.6 Sequences of peptides in the study.

\begin{tabular}{|c|c|c|}
\hline No & Peptide name & Sequence \\
\hline 1 & V2 monomer & H-RGRKVVRRKK- $\mathrm{NH}_{2}$ \\
\hline 2 & V2-AGC & H-RGRKVVRRAGC- $\mathrm{NH}_{2}$ \\
\hline 3 & V2-Cya & H-RGRKVVRR-NH- $\mathrm{CH}_{2}-\mathrm{CH}_{2}-\mathrm{SH}$ \\
\hline 4 & V2-Cys & H-RGRKVVRRC-NH2 \\
\hline 5 & V2 dimer & $(\mathrm{H}-\mathbf{R G R K V V R R})_{2} \mathbf{K K}-\mathrm{NH}_{2}$ \\
\hline 6 & V2-AGC dimer & (H-RGRKVVRRAGC- $\left.\mathrm{CH}_{2} \mathrm{CO}-\right)_{2} \mathbf{K K}-\mathrm{NH}_{2}$ \\
\hline 7 & V2-Cys dimer & (H-RGRKVVRRC- $\mathrm{CH}_{2} \mathrm{CO}-{ }_{2} \mathbf{K K}-\mathrm{NH}_{2}$ \\
\hline 8 & V2-Cya dimer & (H-RGRKVVRR- $\left.\mathrm{NH}-\mathrm{CH}_{2}-\mathrm{CH}_{2}-\mathrm{S}-\mathrm{CH}_{2} \mathrm{CO}-\right)_{2} \mathbf{K K}-\mathrm{NH}_{2}$ \\
\hline 10 & DFTamP1-p & H-GLLPLLSLLGKLL-NH ${ }_{2}$ \\
\hline 11 & DFTamP1-Cys & H-GLLPLLSLLGKLLC-NH ${ }_{2}$ \\
\hline 12 & GPN-dimer & H-GLLPLLSLLGKLLGPNGLLPLLSLLGKLL-NH ${ }_{2}$ \\
\hline 13 & DFTamP1-dimer & H-GLLPLLSLLGKLL(H-GLLPLLSLLGKLL)KK-NH \\
\hline 14 & DFTamP1-Сya & H- GLLPLLSLLGKLL-NH- $\mathrm{CH}_{2}-\mathrm{CH}_{2}-\mathrm{SH}$ \\
\hline
\end{tabular}

The Bold letters indicate amino acid residues; the red part indicates the presence of the thiol entity. V2 monomer, V2 dimer and DFTamP1-p are used as controls. 


\subsubsection{HPLC analysis}

All analytic HPLCs were conducted using Shimadzu reverse phase HPLC system equipped with a C18 (5um, 4x250mm) analytical column (Phenomenex, United States of America) at a flow rate of $1.0 \mathrm{ml} / \mathrm{min}$. Small scale purifications were performed using semi-preparative HPLC column with a Shimadzu system equipped with a C18 reversephase column (5um, 10x250mm) (Phenomenex, United States of America) at a flow rate of $2.5 \mathrm{ml} / \mathrm{min}$. Preparative purifications were performed using Waters system equipped with Alltech ProSphere C18 column (10um, 22x250mm) with a flow rate of $10 \mathrm{ml} / \mathrm{min}$. The buffer system for all the analysis was buffer A and buffer B. The composition of buffer A was $0.045 \%$ TFA in $\mathrm{ddH}_{2} \mathrm{O}$ (double distilled water), and buffer B was $90 \%$ acetonitrile (ACN) (Merck KGaA, Germany), $10 \% \mathrm{ddH}_{2} \mathrm{O}$ and $0.039 \%$ TFA. The chromatogram of the peptide was monitored at a wavelength of $220 \mathrm{~nm}$. Usually, after analysis with analytical RP-HPLC, the crude peptide was purified using semi-preparative or preparative RP-HPLC, on a C18 column. Different elute fractions were collected and analyzed by Matrix Assisted Laser Desorption Ionization Mass Spectrometry (MALDI-MS) to determine the molecular weight of the peptide. $\alpha$-cyano-4-hydroxycinnamic acid (Sigma, India) was used as the matrix for MALDI-MS. The fractions containing pure peptide were lyophilized and stored at $-20{ }^{\circ} \mathrm{C}$.

\subsubsection{Mass spectrometry analysis}

Electrospray ionization mass spectra were acquired using Thermo FINNIGAN LCQ Deca XP MAX equipped with an ESI ion source. Matrix-assisted laser desorption/ionization MS spectra were acquired on Applied Biosystems 4800 MALDITOF/TOF analyzer in Reflective mode. MALDI MS was performed by mixing a sample of $0.5 \mu \mathrm{l}$ semi-preparative HPLC fraction and $0.5 \mu \mathrm{l} \alpha$-cyano-4- hydroxyl cinnamic acid (saturated in aqueous aceonitrile+0.1\% TFA). 


\subsubsection{Different ways of dimerization}

Conventionally peptides are dimerized using lysine as a branching core and coupling the amino acid residues on Lys $\alpha$ and $\varepsilon$ amino groups by SPPS. ${ }^{42}$ We utilized a different approach where peptide chains were joined to a core by a simple conjugation reaction. Basically, it is a reaction between the thiol group of one peptide and a bromoacetyl moiety of another peptide. The substitution reaction takes place to form a covalent linkage between the two peptides. ${ }^{63}$ This reaction can be used for making dimers of the same peptide sequence or conjugating different peptides. For the synthesis of dimers on a bivalent Lys template, the $\alpha, \varepsilon$-dibromoacetylated lysine core was conjugated with the thiol-containing peptide.

The general principle for thiol alkylation-mediated conjugation/dimerization is shown below.

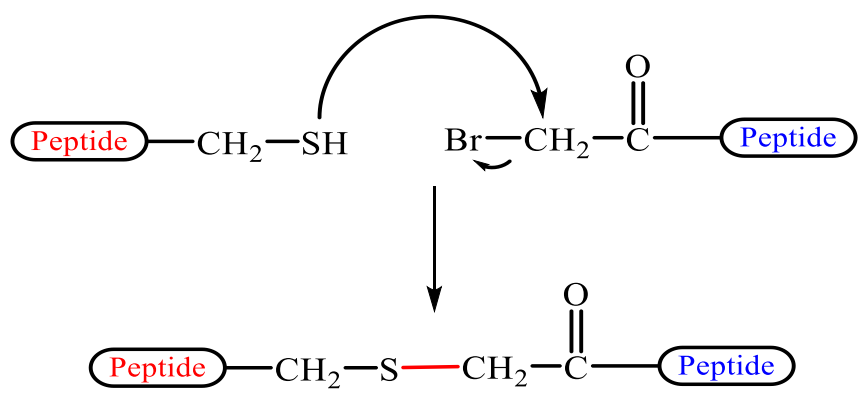

Figure 2.26 Conjugation/dimerization of peptides using the thiol alkylation reaction.

\subsubsection{Preparation of $\alpha, \varepsilon$-dibromoacetyl-Lys-Lys-NH2}

The 0.2 mmole Rink amide MBHA resin was taken. The resin was subjected to $20 \%$ piperidine treatment for 20 mins to remove the Fmoc group. After washing, FmocLys(Boc)-OH was coupled to the resin, followed by Fmoc deprotection and coupling of Fmoc-Lys(Fmoc)-OH. After Fmoc deprotection, $\alpha$ and $\varepsilon$ amino groups of the $\mathrm{N}$-terminal Lys were coupled with bromoacetic acid using DCC/HOBt. Once coupling was complete, 
the peptide was cleaved using standard cleavage solution. It was purified by HPLC, and molecular weight was confirmed by MALDI-MS and lyophilized at $-20^{\circ} \mathrm{C}$.

\subsubsection{Dimerisation of V2-Cys(4) on the bivalent core $\alpha, \varepsilon$-dibromoacetyl- Lys-Lys- $\mathrm{NH}_{2}$}

$10 \mathrm{mM}$ of the di-bromoacetyl lysine core and $22.5 \mathrm{mM}$ of V2-Cys were reacted in a buffer (50\% $\mathrm{ACN}$ in $\mathrm{H} 2 \mathrm{O}$ with $0.045 \% \mathrm{TFA})$. The $\mathrm{pH}$ of the reaction solution was

adjusted to 8 using sodium bicarbonate (Alfa Aesar, United Kingdom) and the reaction was kept in the dark. Analytical RP-HPLC (Gradient: buffer B 0-40\% in 40 mins) was used to monitor the progress of the reaction at different intervals. The reaction time was approximately $24 \mathrm{~h}$. Once the reaction was completed, the desired peptide was purified using C18 semi-preparative RP-HPLC with the same gradient. The fractions were collected and analyzed by MALDI-TOF MS. The fractions containing pure peptide were lyophilized and the dried powder was stored at $-20^{\circ} \mathrm{C}$.

\subsubsection{Dimerisation of V2-Cya(3) with the di-bromoacetyl linker}

$10 \mathrm{mM}$ of the di-bromoacetyl linker and $22.5 \mathrm{mM}$ of V2-Cya was reacted in the same buffer under the same conditions. The reaction was completed in $24 \mathrm{~h}$. After completion of the reaction, the desired peptide was purified using C18 semi-preparative RP-HPLC and analyzed by MALDI-TOF MS. The fractions containing pure peptides were lyophilized and the peptide powder was stored at $-20^{\circ} \mathrm{C}$.

$10 \mathrm{mM}$ of the di-bromoacetyl lysine core and $22.5 \mathrm{mM}$ of V2-Cys were reacted in a buffer $(50 \% \mathrm{ACN}$ in $\mathrm{H} 2 \mathrm{O}$ with $0.045 \% \mathrm{TFA})$. The $\mathrm{pH}$ of the reaction solution was adjusted to 8 using sodium bicarbonate (Alfa Aesar, United Kingdom) and the reaction was kept in the dark. Analytical RP-HPLC (Gradient: buffer B 0-40\% in 40 mins) was used to monitor the progress of the reaction at different intervals. The reaction time was approximately $24 \mathrm{~h}$. Once the reaction was completed, the desired peptide was purified using C18 semi-preparative RP-HPLC with the same gradient. The fractions were collected 
and analyzed by MALDI-TOF MS. The fractions containing pure peptide were lyophilized and the dried powder was stored at $-20^{\circ} \mathrm{C}$.

\subsubsection{Dimerisation of Cys-attached DFTamP1-p(11) with di- bromoacetyl linker}

$10 \mathrm{mM}$ of the di-bromoacetyl-lysine linker dissolved in Buffer A (60\% ACN:40\% $\left.\mathrm{H}_{2} \mathrm{O}\right)$ and $22.5 \mathrm{mM}$ of DFTamP1-Cys dissolved in buffer $\mathrm{B}\left(70 \% \mathrm{ACN}: 30 \% \mathrm{H}_{2} \mathrm{O}\right)$ were mixed. The $\mathrm{pH}$ of the reaction mixture was adjusted to 7.5 using sodium bicarbonate (Alfa Aesar, United Kingdom) and the reaction was kept in the dark. Analytical RP-HPLC was conducted to monitor the reaction (Gradient: buffer B 0-100\% 50 mins). After $24 \mathrm{~h}$, the desired peptide was purified using C18 semi-preparative RP-HPLC with the same gradient; fractions were collected and analyzed by MALDI-TOF MS. The fractions containing pure peptides were lyophilized and the lyophilized powder was stored at $-20^{\circ} \mathrm{C}$.

\subsubsection{Radial diffusion assay}

Radial diffusion assay was used to determine antibacterial activity of the peptides. This method is highly sensitive and consumes a minimal amount of the sample preparations being tested. It uses microbial cells grown to mid-log phase, entrapped within thin gels containing limited nutrients. Peptides were introduced into small wells, from which they diffused radially into the gels. After 3 hours of incubation nutrient-rich top agar was poured into the plate with thin gel for additional colony development. A zone of inhibition was measured, and the results were analyzed by plotting data on a graph. The assay can be done on many different bacteria with essentially any antibacterial agents.

To prepare organisms for the assay, a single colony was picked with a polystyrene loop, transferred to a $125-\mathrm{mL}$ bottle that contains $50 \mathrm{~mL}$ of TSB, and incubated at $37{ }^{\circ} \mathrm{C}$ in a shaking water bath for $18-24 \mathrm{~h}$. An aliquot $(50 \mu \mathrm{L}$ of $E$ coli or desired strain) of the resulting stationary phase cultures was transferred to $50 \mathrm{~mL}$ of fresh TSB and incubated for $2.5 \mathrm{~h}$ at $37^{\circ} \mathrm{C}$ in a shaking water bath. Around $5 \mathrm{~mL}$ of mid-log phase culture was 
collected and washed thrice with $10 \mathrm{mM}$ phosphate buffer $\mathrm{pH}$ 7.4, and resuspended in 5 $\mathrm{mL}$ of the same cold buffer. One milliliter was removed to measure its optical density at $620 \mathrm{~nm}$. From this information, the concentration of bacteria in the remaining $4 \mathrm{~mL}$ was calculated from the following formula, $\mathrm{CFU} / \mathrm{mL}$ (Colony forming units $/ \mathrm{mL}$ ) $=\left.\mathrm{OD}\right|_{620} \mathrm{X} 2.5$ $\mathrm{X} 10^{\wedge} 8$. From this calculation, the volume of washed bacterial suspension that contains 4 $\mathrm{x} 10^{\wedge} 6 \mathrm{CFU}$ (the inoculum used for each underlay) can be determined.

A $10-\mathrm{mL}$ aliquot of the sterile molten underlay agar was transferred to a $15-\mathrm{mL}$ conical plastic centrifuge tube. The medium was inoculated with $4 \times 10^{\wedge} 6 \mathrm{CFU}$ of washed bacteria, inverted few times, and then poured into a $10 \times 10 \times 15 \mathrm{~cm}$ square dish on a leveling table, where it solidified in less than $2 \mathrm{~min}$, and then the Petri dishes were labeled.

After the underlayer gel has set, an array of $2 \mathrm{~mm}$ wells was punched. The central plugs were removed by suction, using a mikura suction pipette. 5-uL aliquots of the various samples to be tested, typically diluted in $0.01 \%$ acetic acid, were added to each well in turn. The plates were covered, turned gel-side up, and incubated for 3 hours in a $37^{\circ} \mathrm{C}$ incubator. Next, each underlay gel was covered with a $10-\mathrm{mL}$ overlay of nutrient-rich overlay agar. As soon as the overlay gel solidified, the plates were recovered and placed gel-side up and incubated at $37^{\circ} \mathrm{C}$ overnight. The following morning, the plates were removed, and $10 \mathrm{~mL}$ of a disinfecting solution (e.g., $5 \%$ acetic acid in $25 \%$ methanol) was applied to the agar surfaces for at least 20 mins before the zone diameters were measured and recorded.

\subsubsection{Calculation of MIC}

The diameter of the zone of inhibition was measured to the nearest $0.5 \mathrm{~mm}$. The average diameter, excluding the $2 \mathrm{~mm}$ diameter of the well, was calculated from three duplicates. A graph of the zone of inhibition diameter against $\log _{10}$ of concentration was plotted. The MIC, which is the lowest concentration that inhibits visible growth of bacteria, was determined from the $\mathrm{x}$-intercept of the line of best fit from the graph. 


\section{CHAPTER 3 \\ VANCOMYCIN C-TERMINAL AMIDE \\ ANALOGS}

\subsection{Introduction of glycopeptides}

Glycopeptide antibiotics (GA) are substrate ${ }^{23}$-binding antibiotics. They were introduced to clinics 50 years ago. ${ }^{71}$ The most commonly used and extensively studied member of glycopeptide antibiotics is Vancomycin. It was isolated from Amycolatopis oriental in 1953 by Edmund Kornfeld. ${ }^{72}$ Vancomycin also serves as a model system to investigate the glycopeptide mode of action, since other GAs kills bacterial cells in a similar way as vancomycin. Vancomycin was initially used for patients allergic to beta-lactam antibiotics. ${ }^{73}$ Later on, due to the prevalence of drug resistance, it was more often used for infections caused by bacterial strains resistant to other antibiotics.

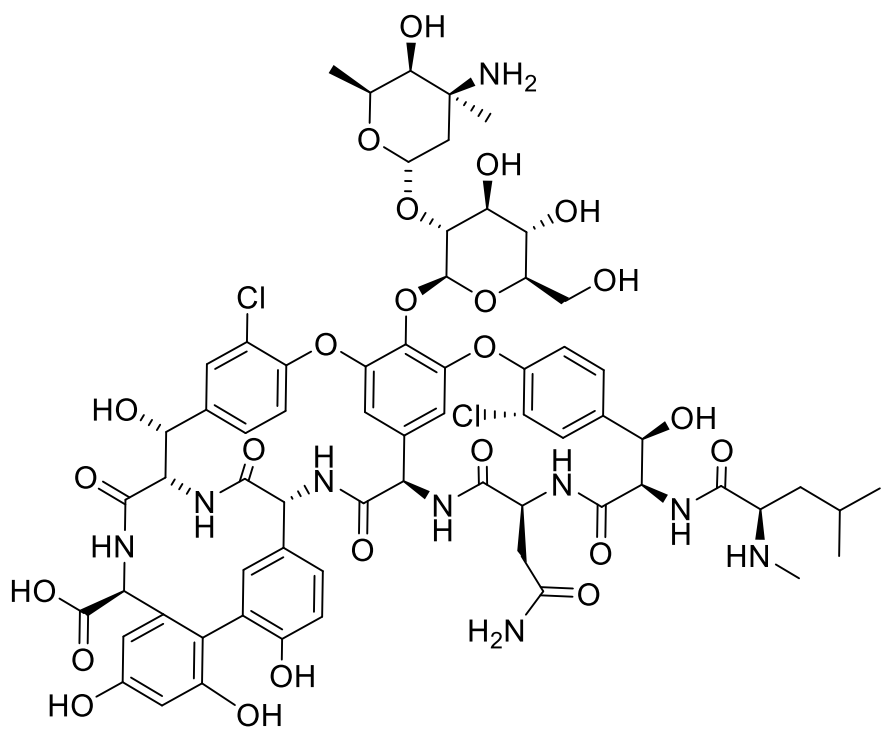

Figure 3.1 The structure of vancomycin. It has a disaccharide (vancosamine and glucose) moiety and a heptapeptide scaffold. 


\subsubsection{Mode of action}

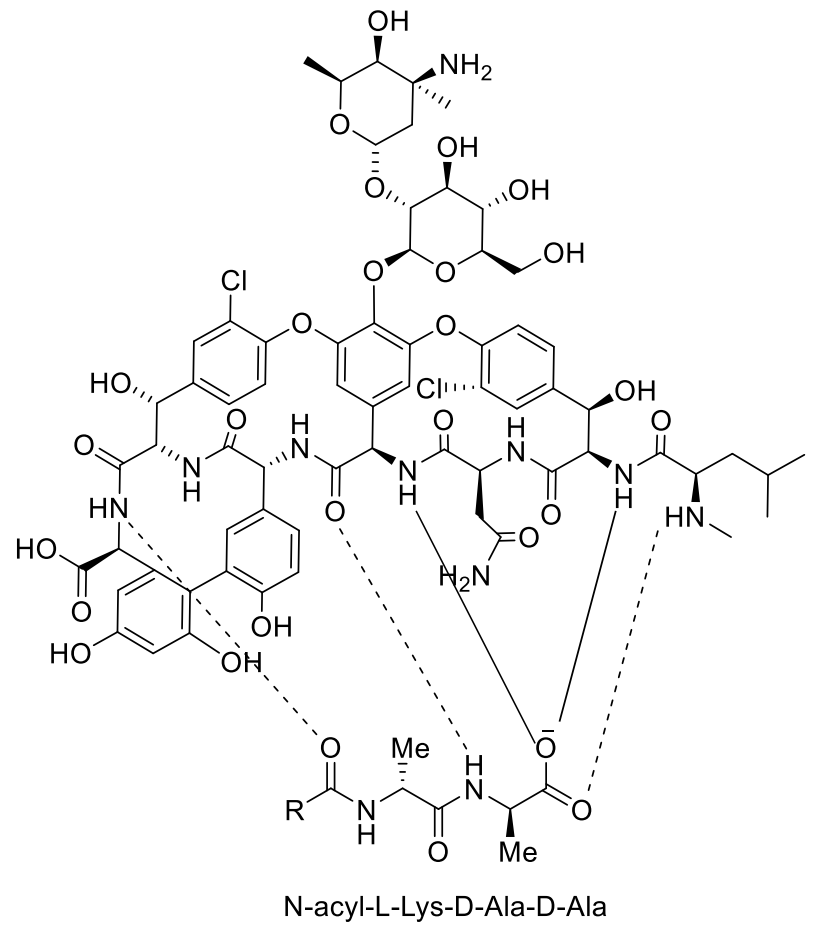

Figure 3.2 The complex between the precursor peptidoglycan terminal peptide N-acyl-L-Lys-D-Ala-DAla-OH and vancomycin binding pocket through five hydrogen bonds.

As for all glycopeptide antibiotics, vancomycin blocks the later stage of peptidoglycan biosynthesis of bacterial cell wall. Biosynthesis of peptidoglycan happens in three stages. In the first stage, pentapeptide precursors of bacterial peptidoglycan are fixed on a di-sugar molecule in the cell. Then in the second stage, these precursors are coupled to a lipid carrier which leads to the transfer of the resulting lipid-peptide molecule to the outer surface of the cytoplasmic membrane. In the third stage, the individual precursors are cross-linked by transpeptidation and transglycosylation reactions. Vancomycin targets this step by binding with a dipeptide D-Ala-D-Ala moiety located at the peptidoglycan C-terminal (Figure 3.2). The binding interactions occur through five hydrogen bonds formed between the D-Ala-D-Ala dipeptide of the peptidoglycan precursor and the heptapeptide backbone of the vancomycin. Once bonded to D-Ala-DAla, vancomycin sterically blocks the transglycosylation and transpeptidation processes, 
preventing the essential cross-linking of peptidoglycan and resulting in weakening of the peptidoglycan layers of the cell wall. Thus, because of osmotic pressure, the bacterial cells become susceptible to lysis. $^{74}$

\subsubsection{Resistance to vancomycin}

In 1988, the first case of vancomycin resistance was reported with enterococci. ${ }^{75}$ It happened after three decades since its introduction to the medical world during which time vancomycin was the drug of the last choice for life-threatening infections. After enterococci, vancomycin resistance quickly spread to the other families of gram-positive bacteria. $^{74}$

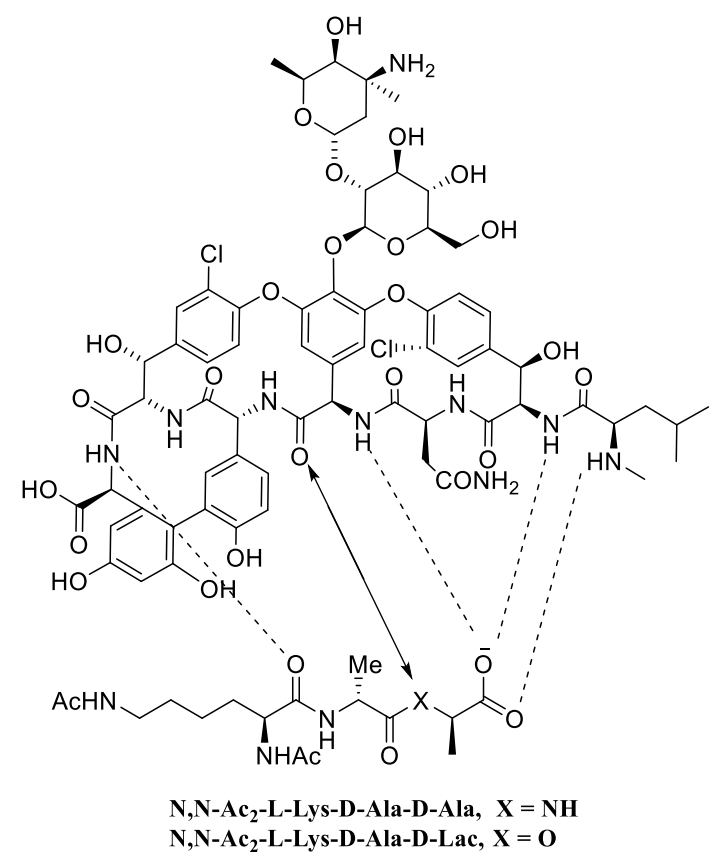

Figure 3.3 Hydrogen bond interactions between vancomycin and the C-ter dipeptide of the peptidoglycan. Note that one hydrogen bond is lost for D-Ala-D-Lactate substrate from some vancomycin-resistant strains.

Since then, its efficacy has been increasingly compromised due to the prevalence of vancomycin-resistant isolates. It is quite a concern because the drug of choice for decades to combat drug-resistant infection is no longer effective. Many resistant strains 
change the peptidoglycan binding substrate of vancomycin from D-Ala-D-Ala to D-AlaD-Lactic acid. Change of Alanine to Lactic acid removed one hydrogen bond between the dipeptide substrate and vancomycin (Figure 3.3). As a result, its binding affinity toward the D-ALa-D-Lac substrate drops by 1000 times. ${ }^{76}$ In some other isolates, the terminal dipeptide is changed to D-Ala-Ser.

\subsubsection{Structure-activity relationship}

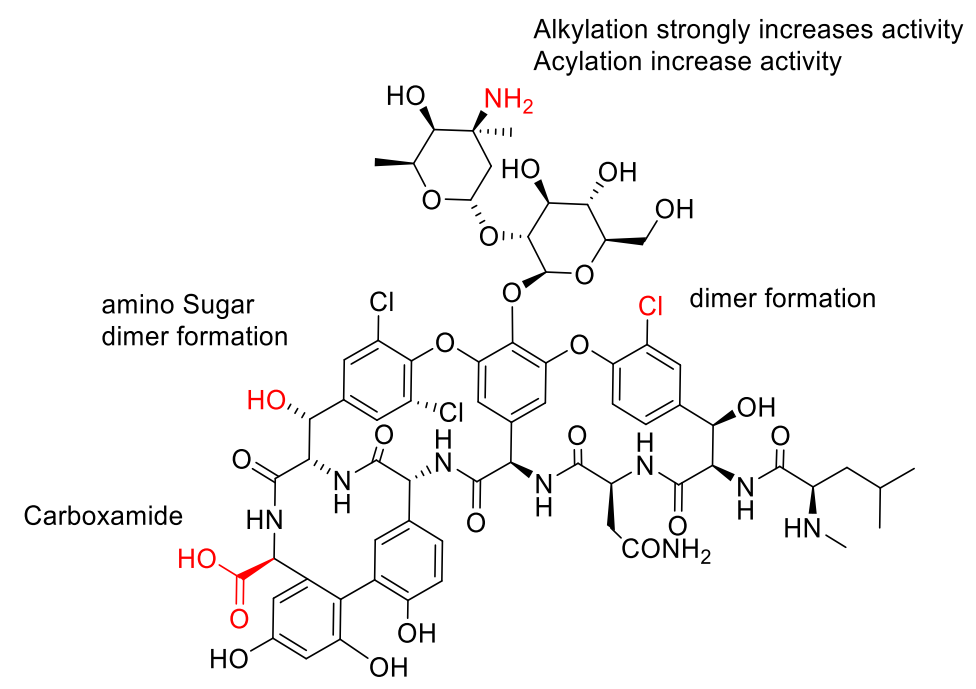

Figure 3.4 Functional sites on vancomycin for structural modification to synthesize analogs.

The molecular mode of interaction between vancomycin and its target reveals several sites in the structure of vancomycin which can influence the activity of the molecule. Many research groups synthesized different analogs by targeting the functional groups of vancomycin at these sites for modification. Some synthesized analogs have better activity than the parent compound, and some are even effective against vancomycinresistant strains. ${ }^{77}$

kahne and cowokers ${ }^{78}$ found the importance of hydrophobic moieties appended with the disaccharide on residue 4 of vancomycin backbone. These hydrophobic moieties can make the analogs effective against drug-resistant strains including VRE. However, these 
lipophilic modification imparts undesired characteristics like poor clearance from the body and accumulation in certain tissues.

Nagaranjan et al. ${ }^{79}$ from Eli Lilly showed that $N$-alkylation of the amino sugar moieties on vancomycin could enhance the antibacterial mode of action. Studies also revealed that $N$-alkylation is more effective than $N$-acylation in improving antibacterial activity. The study concluded that vancosamine mono-alkylated derivatives are more potent than the parent compound. ${ }^{80}$

Thompson and coworkers ${ }^{80}$ synthesized potent vancomycin derivatives, and FDA recently approved one of the analogs for clinical use. The study by Nagaranjan et al. ${ }^{79}$ already concluded that $N$-alkylation of vancosaminyl moiety of vancomycin could increase its antibacterial effect towards vancomycin-resistant bacteria. ${ }^{80}$ In their study, they chose one of the vancomycin derivatives, which has appended sugar moiety at benzylic hydroxyl group of residue 6 . They conducted reductive alkylation on the derivatives, where the amine from vansoamine reacted with 4'-chloro-biphenyl-carboxaldehyde. The resultant product is 50 folds more potent than the parent compound against resistant strains. The alkylation improves the antibacterial activity of vancomycin derivatives which rattles the functional integrity of the bacterial cell wall.

Arimoto and co-workers ${ }^{81}$ synthesized vancomycin analogs by the replacement of $\mathrm{Cl}$ groups using Suzuki-Miyaur cross-coupling reaction. The analogs were synthesized by replacement of $\mathrm{Cl}$ at residue 6 and residue 2 of vancomycin with different carbon substituents. Interpolation of the substituent at residue 2 of vancomycin improved its antibacterial activity even against vancomycin-resistant strains, whereas the modification at the residue 6 diminished its activity even against vancomycin-sensitive bacteria. In this way, the importance of $\mathrm{Cl}$ at position 2 of vancomycin and its replacement with styryl substituted was established to synthesize potent analogs against VRSA and VRE. ${ }^{81}$

Kevin Judice and co-workers ${ }^{82}$ synthesized a series of lipidated vancomycin analogs that contain disulfide bonds within their lipid chains. Their work was focused on 
improving pharmacokinetic parameters. They developed disulfide analogs that could be metabolized to hydrophilic derivative inside the body and eliminated through urine. The disulfide bonds could allow their analogs to self-dimerize and form vancomycin disulfidelinked dimers. These disulfide lipophilic analogs exhibited enhanced pharmacokinetic parameters. $^{82}$

Nishitini et al. ${ }^{83}$ examined a vancomycin-related glycopeptide antibiotic, chloroorienticin B, and synthesized series of $\mathrm{N}$-alkylated and aminomethylated derivatives. It was anticipated that the derivatives with hydrophobic $\mathrm{N}$-alkylate moiety and hydrophilic amino-methylated moiety provided vital acceleration in improving antibacterial activity against MRSA and VRE. This methodology is considered as an effective way to discover efficient semi-synthetic glycopeptide antibiotics. ${ }^{83}$

Preobrazhenskaya and co-workers ${ }^{84}$ examined the effect of $\mathrm{N}$-acylation and nitrosylation at the $\mathrm{N}$-terminus of vancomycin. They demonstrated that nitrosylated derivatives exhibited higher antibacterial activity whereas acylated derivatives were not equally potent. They also exploited the C-terminal of vancomycin derivative eremomycin to make potent analogs. Hydrazine and similar moieties were reacted with vancomycin to form carboxamide and hydrazide derivatives. Some analogs have either comparable or slightly better activities than the parent compound. The carboxyl group was recommended to be an important site of modification for the discovery of more potent analogs. ${ }^{84}$

Griffin and coworkers ${ }^{85}$ reported the first exploitation of carboxyl group of vancomycin for analog synthesis. They synthesized a series of vancomycin derivatives which can bind to the peptidoglycan precursors. However, they have not conducted any SAR or antimicrobial studies for these analogs. ${ }^{85}$

Similarly, Haldar and coworkers ${ }^{76}$ synthesized carboxamide derivatives of vancomycin. They synthesized lipophilic cationic vancomycin analogs, and some of the analogs they synthesized were quite active against vancomycin susceptible strains. One of the analog with comparatively long carbon chain exhibited potent antibacterial activity 
against vancomycin-resistant Enterococci (VRE). The study showed that the carboxyl group of vancomycin could be used to develop potent analogs with optimized charge and lipophilic ratio. ${ }^{76}$

Boger and coworkers ${ }^{86}$ have conducted numerous studies on total chemical synthesis of vancomycin and its analogs. They published various studies focused on Nterminal modification of vancomycin, modification of the binding pocket, vancomycin analogs with and without sugar moieties. In their recent work on the total chemical synthesis of a new vancomycin analog, the oxygen atom from the carbonyl group of residue 4, which is a key hydrogen bond acceptor in the binding pocket, was replaced. This particular oxygen cannot form a hydrogen bond with a vancomycin-resistant substrate of D-Ala-D-Lac. However, when the oxygen was replaced with $\mathrm{NH}$, the resultant amidine analog revived its lost binding activity with the substrate, amidine acting as a hydrogen bond donor can form a hydrogen bond with the oxygen of D-lactic acid. The bonding interaction is shown in Figure 3.5. Z1 shows the standard structure of vancomycin and Z2 is the amidine analog. It was a challenging work to synthesize analogs with altered binding pocket, which represented a huge development in this field. ${ }^{77}$

\subsubsection{The focus of this study}

Total synthesis is extremely challenging to prepare vancomycin analogs. Modifying the vancomycin molecule directly represents a more practical way of deriving its analogs. However, multi-step reactions are usually needed to achieve the desired modifications. Therefore, a simple and efficient strategy which can introduce functionalities into the vancomycin structure in a single step will be quite useful.

The focus of the project is to synthesize potent vancomycin analogs through a simple chemical scheme. The emphasis is on devising an efficient methodology which allows the incorporation of new functional groups, particularly amines, onto vancomycin to increase its net positive charges. We focus on the C-terminal carboxyl group of vancomycin and use it to react with diamines: one amine to form a carboxamide, the other 
amine to bring a new positive charged group to vancomycin. We hope that this will improve the antibacterial activity because it may bring additional interactions with the lipidpeptidoglycan precursor or help deliver the large vancomycin molecule to its site of action through favorable interactions with the bacterial cell surface. This strategy of modifying the C-terminal carboxyl was also used to modify other glycopeptides, such as teicoplanin and potent carboxamide derivatives were obtained. ${ }^{87}$ Nevertheless, modifying the carboxyl group of vancomycin has been underutilized in developing vancomycin analogs. The amide formation chemistry is a simple single-step reaction, capable of deriving a large number of analogs in a very efficient way.

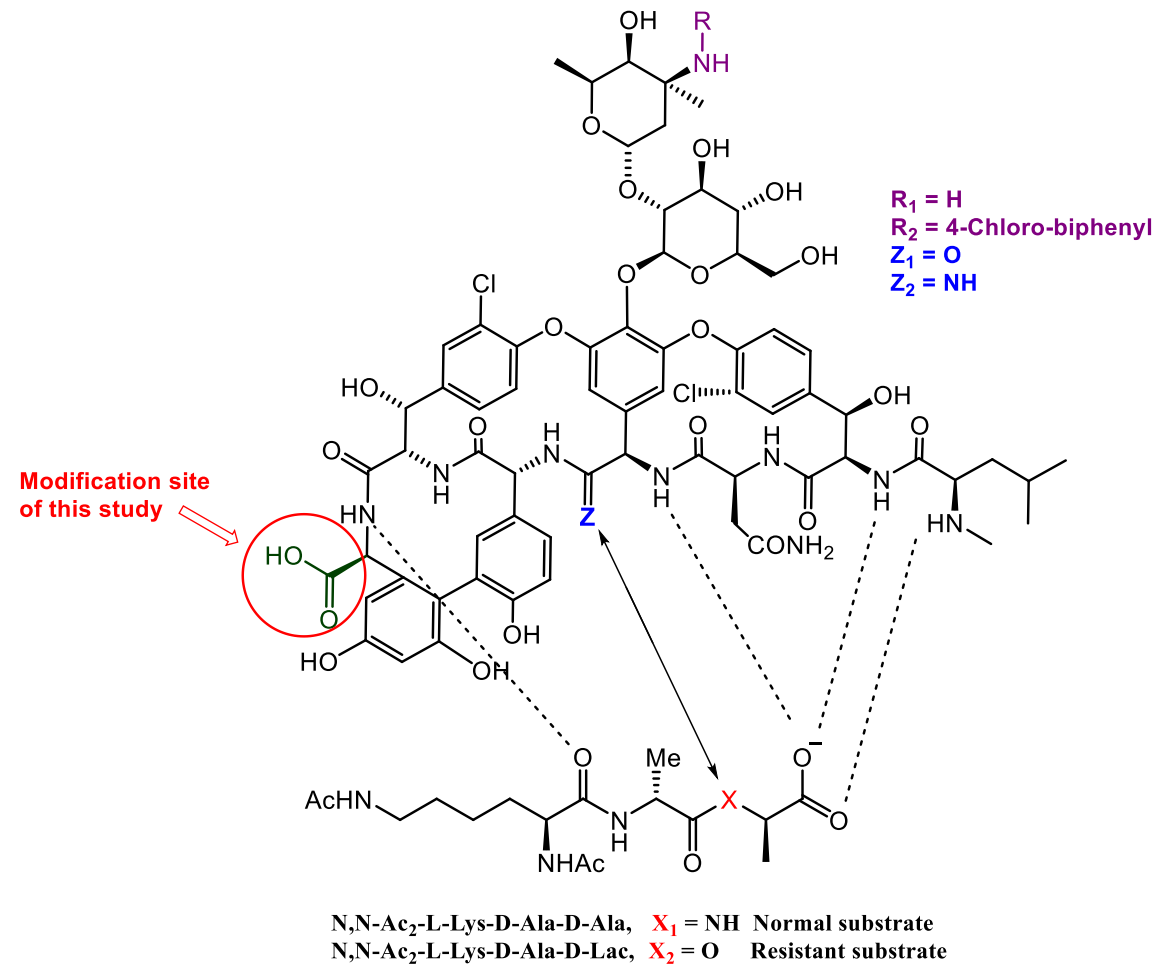

Figure 3.5 Some modification sites in vancomycin. The red circled carboxyl is used in this study. 


\subsection{Results and Discussion}

\subsubsection{Design principle}

Vancomycin and other glycopeptides induce killing by targeting the lipidpeptidoglycan precursor of bacterial cell wall. It is very difficult for bacteria to change the structure of this target site. By targeting this site, the glycopeptide antibiotics were able to evade drug resistance for several decades. However, bacteria managed to acquire resistant genes and change the binding substrate, which renders most of the glycopeptide antibiotics less effective or ineffective. Therefore, developing potent new molecules with enhanced antibacterial and pharmacological activity is needed to combat the drug-resistant problem. The idea is that although resistance against the new analogs may still occur, the development of such resistance will be slow.

The aim of this project is to develop a scheme for the synthesis of various analogs by modifying the C-terminus of vancomycin. The carboxyl group of vancomycin is not involved in forming the complex with its peptidoglycan substrate. ${ }^{88}$ It is expected that modification at the carboxyl group will retain the original activity of the parent compound. It is also expected that this carboxylate can be selectively activated. As shown in some previous studies, modification at this site can improve the antibacterial or pharmacological profile of the analogs.

The chemistry of amide synthesis is employed to form various analogs. Here, we have chosen simple amines, mostly diamines, in our study to prepare the amide derivatives of vancomycin. The presence of an additional amino functional group can interact with negatively charged phospholipid in an interesting way. Previous groups demonstrated improvement in activity of analogs with the addition of such moieties. Some carboxamide derivatives of other glycopeptides show better antibacterial profile than the parent compound. The project aims to further explore the potential of similar amide derivatives at C-terminus of the heptapeptide scaffold of vancomycin. 
A.

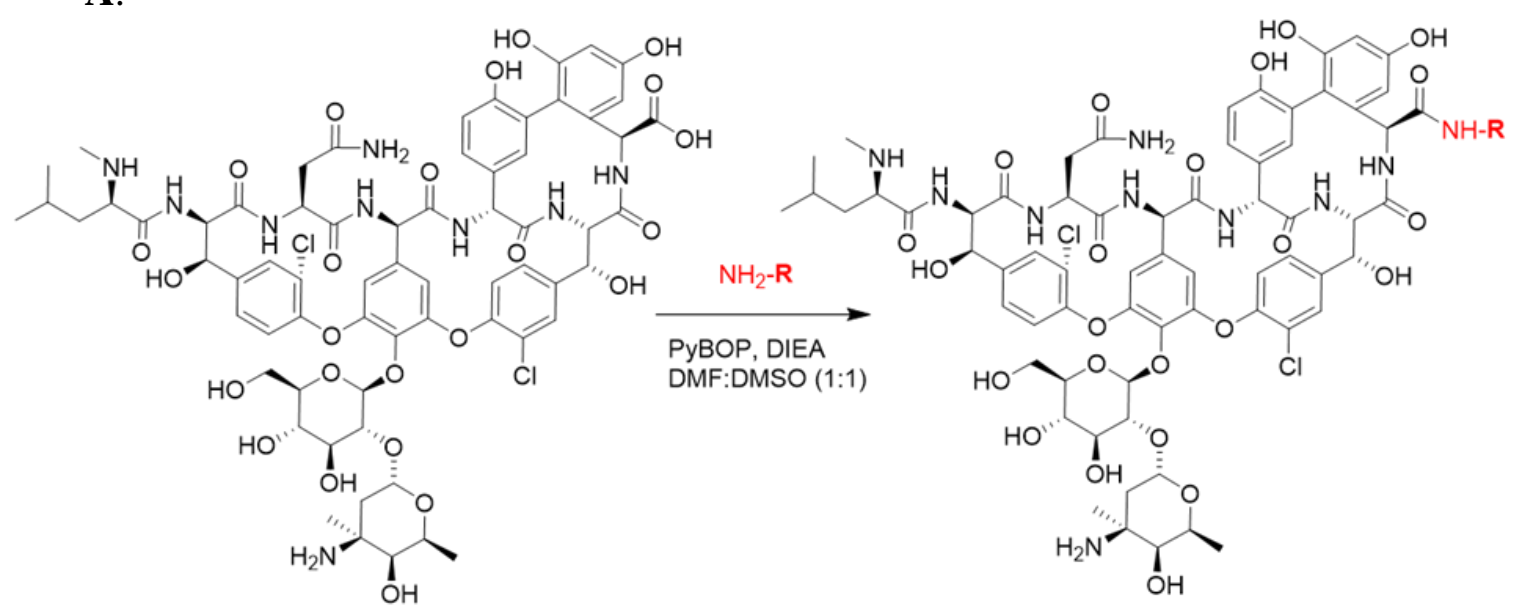

B. List of amine compounds used for carboxamide formation:
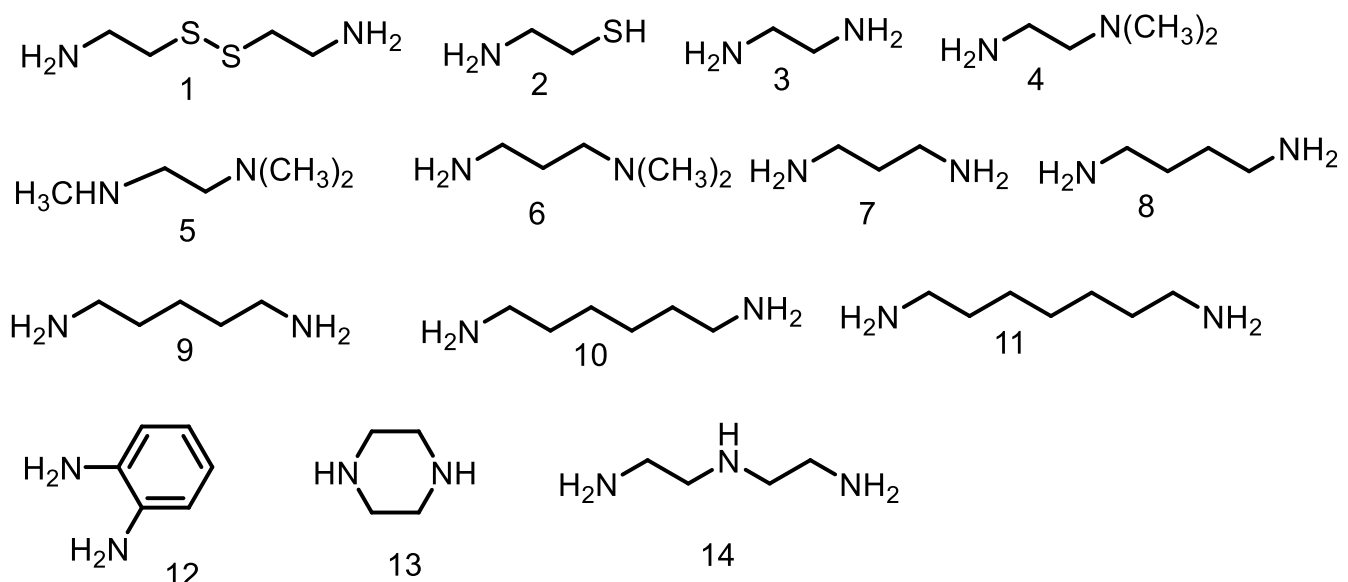

Figure 3.6 A. The general scheme of reaction between the carboxyl group of vancomycin and an amine.

B. List of amines used to prepare vancomycin C-terminal amide derivatives.

\subsubsection{Synthesis of the carboxamide analogs of vancomycin}

The carboxyl group of vancomycin can couple with various amine-containing compounds (Figure 3.6). PyBOP was used as the activating agent. Different solvents mixture were used to solubilize the reactants. A combination of DMF and DMSO (1:1) was found to be optimal for the reaction. In this study, 14 amine compounds were chosen for 
preparing the carboxamide derivatives, most of them diamines (Figure 3.6B). The linear diamines form a series of homologous basic compounds with the carbon chain length ranging from two to seven Cs. Two cyclic diamines, one aromatic (compound 12) and one aliphatic (compound 13), were also employed to determine the effect of the ring structure and basicity on the activity. Compound 2, i.e., cysteamine, is a monoamine whereas compound 14 is a triamine. The lipophilicity of the carbon chain and the presence of the basic amino group impart a major role in the pharmacological profile of the glycopeptide. The hydrophobic moieties can impart additional mechanism of action to the glycopeptides, as reported for teicoplanin analogs. ${ }^{89}$

The synthesis of these analogs used a large excess of the amine compounds versus vancomycin to drive the reaction to completion with only monoacylation. As a general procedure, pre-lyophilized vancomycin, the amine compound and PyBOP were mixed at a ratio of 1: 20-50 : 2-5 in the reaction solution. Varying amount of DIEA was added depending on the amine compound used (salt form or free amine). For many of the diamines, DIEA was not used because the diamine is basic itself and was used in excess. Solubility was an issue associated with vancomycin in these reactions. Ultrasonication was needed in some cases. The reaction mixture incubated at room temperature for about $1.5-$ $2 \mathrm{~h}$. Then the organic solvents were removed by lyophilization, and the residue was redissolved in an aqueous solution and subject to reverse-phase HPLC purification. Similar to teicoplanin amide analogs, we did not observe any significant epimerization of the C-ter residue presumably because oxazolone formation was unfavored due to ring constraint imposed by the cyclic structure formed by the three C-terminal residues.

The purified products were analyzed by reverse-phase HPLC, characterized by mass spectrometry and lyophilized. The final products were obtained as TFA salt after HPLC purification. The analogs synthesized are numbered from 1 to 14 accordingly to the 14 amines used (Figure 3.6B). 


\subsubsection{Synthesis of 1 (Van-cystamine)}

The above general procedure was used to prepare vancomycin-cystamine by using cystamine dihydrochloride $\left(\mathrm{H}_{2} \mathrm{NCH}_{2} \mathrm{CH}_{2} \mathrm{SSCH}_{2} \mathrm{CH}_{2} \mathrm{NH}_{2} .2 \mathrm{HCl}\right)$ for coupling with vancomycin. The ratio of vancomycin/cystamine hydrochloride/PyBOP/DIEA was 1/20/5/60. Figure 3.7 shows the structure of the product Van-cystamine 1. Figure 3.8 shows the analytical HPLC profile and ESI-MS spectrum of the purified product.

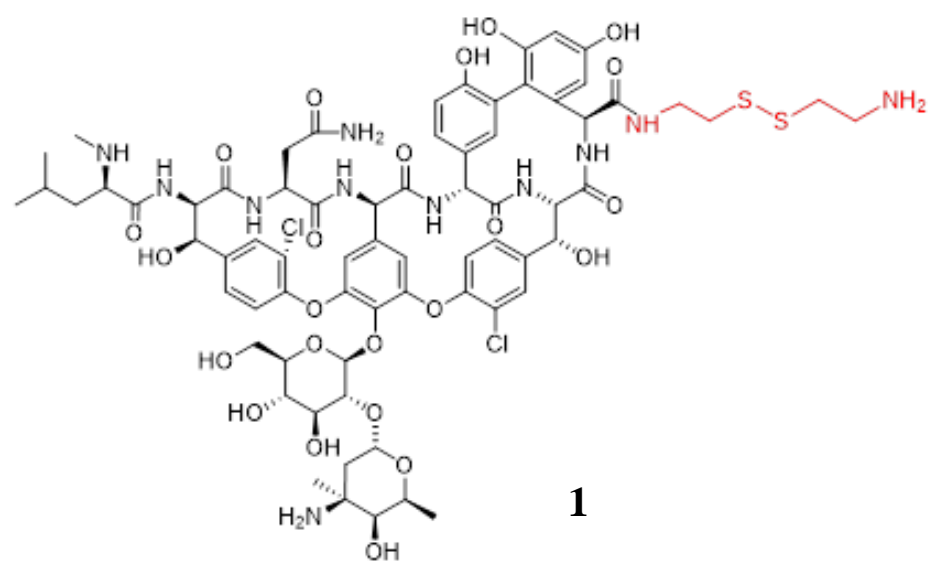

Figure 3.7 The structure of Van-cystamine 1.
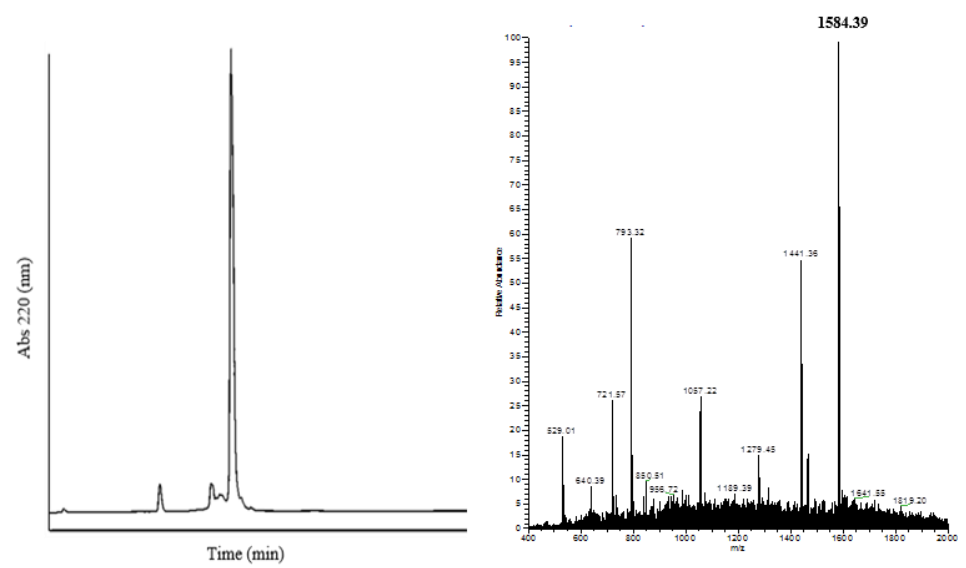

Figure 3.8 Analytical HPLC profile (left panel) and the ESI-MS spectrum (right panel) of the purified Vancystamine analog 1 . Observed $\left[\mathrm{M}+\mathrm{H}^{+}\right]^{+}=1584.39$. 


\subsubsection{Synthesis of 2 (Van-cysteamine)}

The general method was also employed for the synthesis of vancomycincysteamine 2 . The reaction gave some side products, as cysteamine has an unprotected thiol. The free thiol group usually causes oxidation reactions which affect the final yield.

Van-cysteamine 2 can also be obtained by reduction of Van-cystamine 1. 1 was subjected to TCEP treatment to reduce the disulfide bond and afford $\mathbf{2}$. TCEP can reduce the disulfide bridge selectively and effectively. ${ }^{90}$ The reduction was completed within a few minutes. Post-reduction HPLC showed the compound is in monomeric form with a free thiol at the terminal of carboxamide moiety. Figure 3.9 shows the structure of Vancysteamine 2, the analytical HPLC profile and ESI-MS spectrum of the purified product.
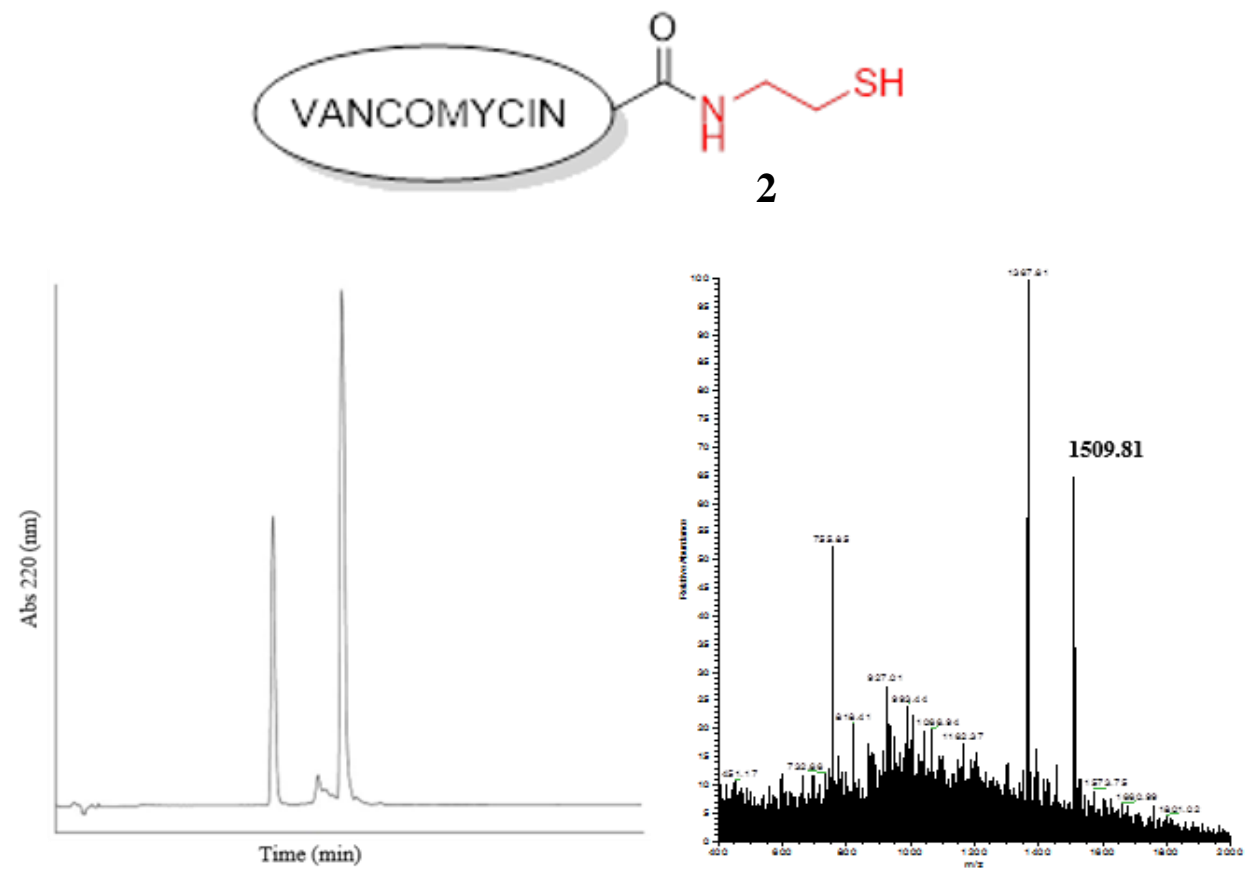

Figure 3.9 Top panel: Structure of Van-cysteamine. Bottom panel: Analytical HPLC profile (left) and the ESI-MS (right) of the purified Van-cysteamine analog 2. Observed $\left[\mathrm{M}+\mathrm{H}^{+}\right]^{+}=1509.81$.

Vancysteamine with unprotected thiol group can be used for conjugating it other novel compounds. Thiol group on the analog allows its conjugation with other antibacterial compounds to synthesize hybrid antibacterial structure that may possess synergistic effect. 


\subsubsection{Synthesis of 3 (Van-ethylenediamine)}

Ethylenediamine is a two-carbon diamine. Because it was used in large excess to vancomycin, the diacylated product was not observed in a significant amount. The additional free amine caused earlier elution of the analog on analytical HPLC than vancomycin.

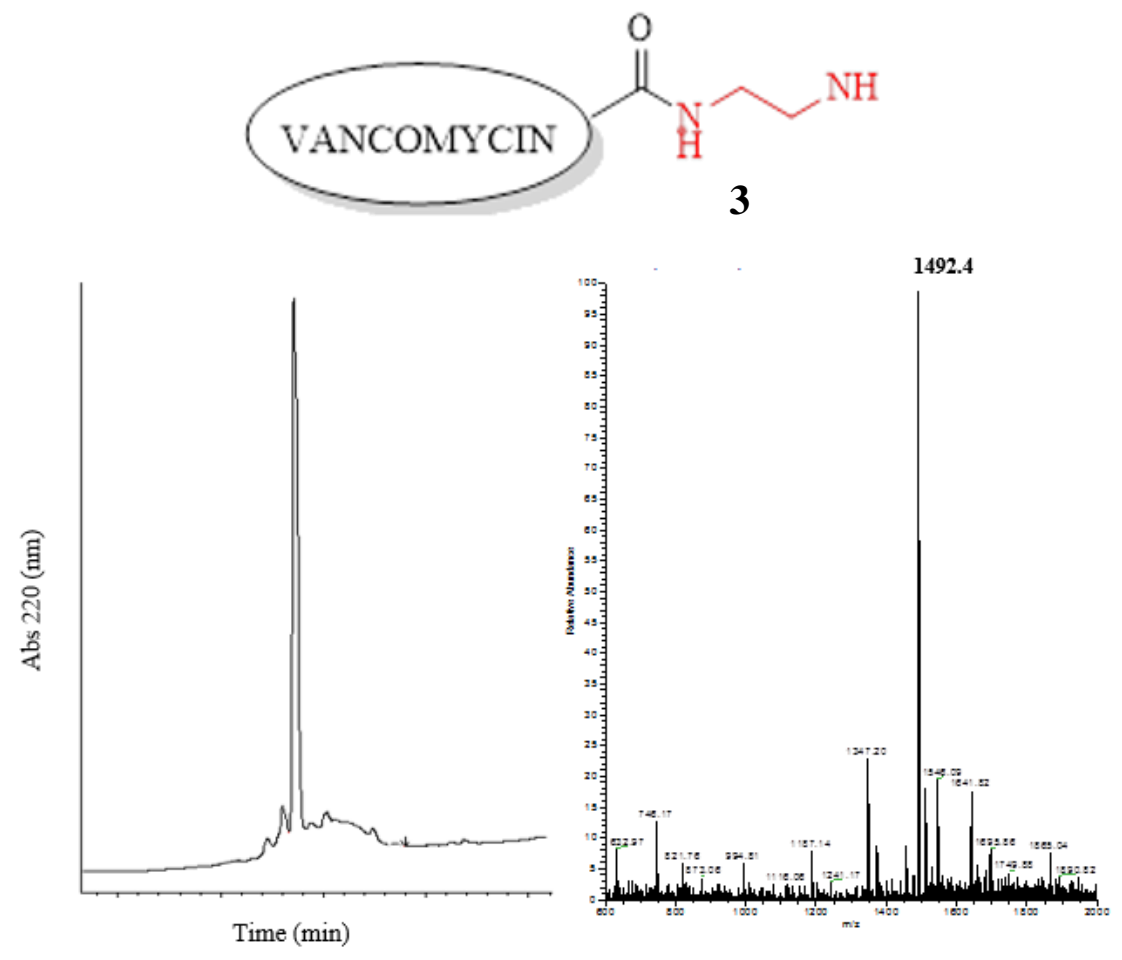

Figure 3.10 Top: Structure of Van-ethylenediamine. Bottom: Analytical HPLC profile (left panel) and the ESI-MS (right panel) of the purified Van-ethylenediamine 3. Observed $[\mathrm{M}+\mathrm{H}]^{+}=1492.4$. 


\subsubsection{Synthesis of 4 (Van-dimethylethylenediamine)}

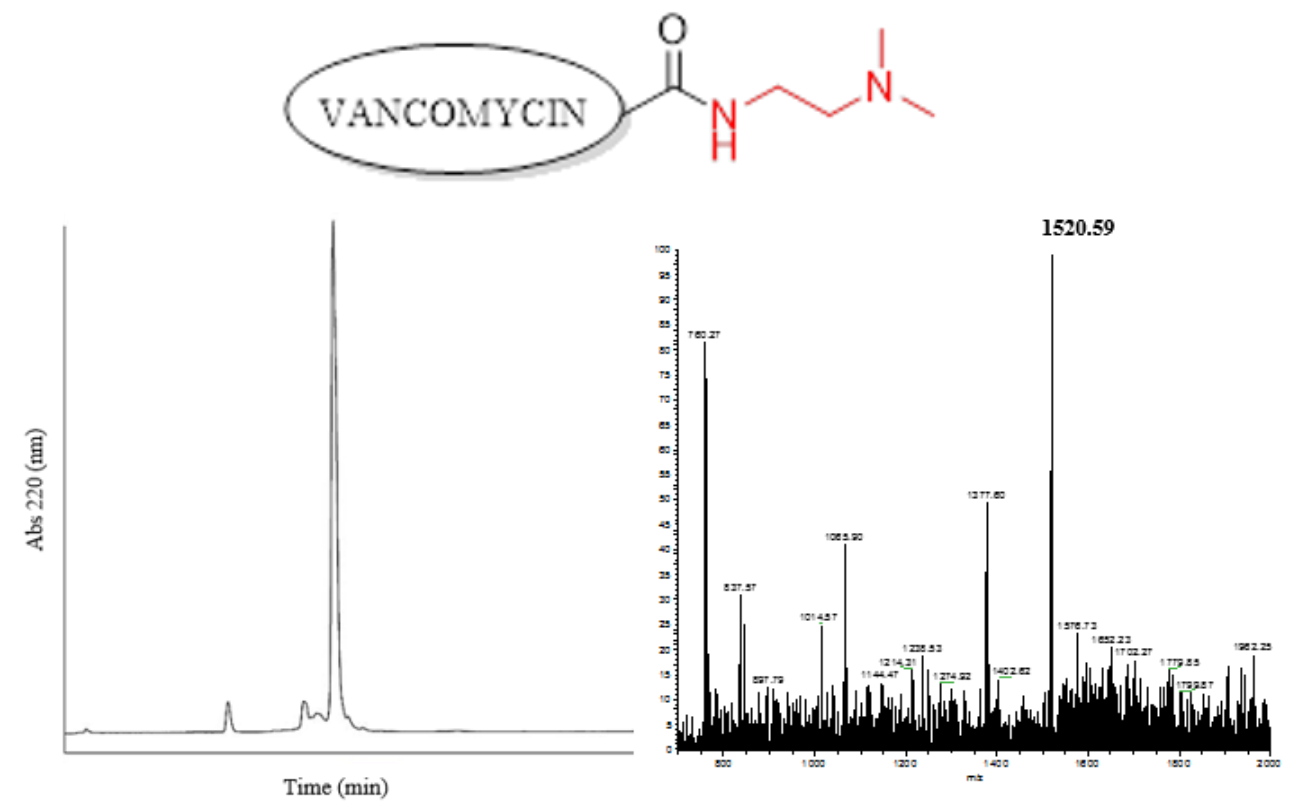

Figure 3.11 Analytical HPLC profile (left panel) and the ESI-MS (right panel) of the purified N, N, dimethyl ethylene diamine analog 4 . Observed $\left[\mathrm{M}+\mathrm{H}^{+}\right]^{+}=1520.59$.

After synthesis of the ethylenediamine derivative 3, the analog with dimethyl substitution on the terminal free amine, Van-dimethylethylenediamine 4, was synthesized. Dimethyl substitution can provide insight to the effect of N-substitution on the activity. 


\subsubsection{Synthesis of 5 (Van-trimethylethylenetriamine)}

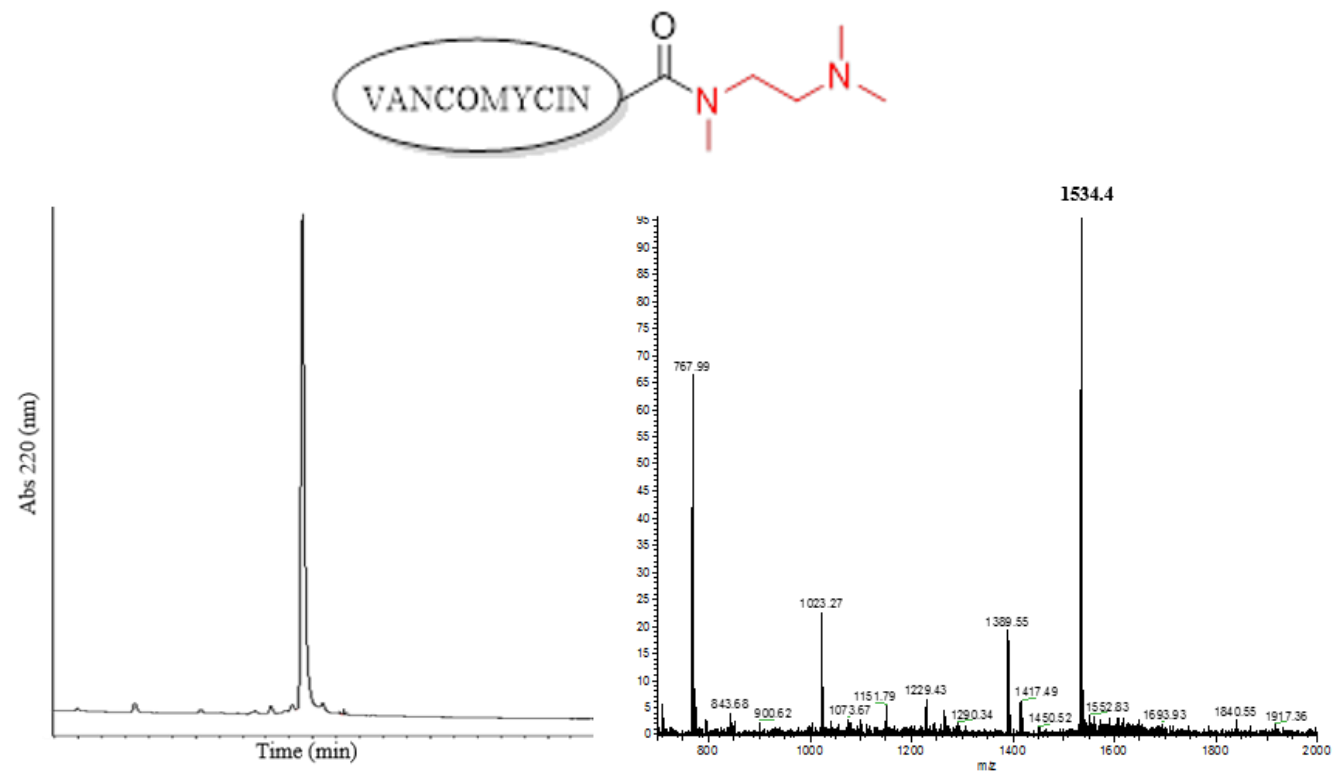

Figure 3.12 Analytical HPLC profile (left panel) and the ESI-MS (right panel) of the purified N, N, Ntrimethyl-ethylene-triamine analog $\mathbf{5}$. Observed $\left[\mathrm{M}+\mathrm{H}^{+}\right]^{+}=1534.4$.

This analog is a tertiary amide in which the amide nitrogen bears a methyl group. The formation of this tertiary amide was not an issue during the synthesis probably because of the large excess of $N, N, N$-trimethyl ethylenediamine used in the reaction.

\subsubsection{Synthesis of $6,7,8,9,10$ and 11}

After synthesis of ethylene diamine analogs, more analogs were synthesized using diamines with longer carbon chains in between the amino groups. These analogs are vancomycin amides formed with 3-dimethylamino-1-propylamine 6, 1,3-diaminopropane 7, 1,4-diaminobutane 8, 1,5-diaminopentane 9, 1,6-diaminohexane $\mathbf{1 0}$ and 1,7diaminoheptane 11. The carbon chain length of the diamines range from 3 to 7 carbon atoms. Except for 6 which has one primary and one tertiary amine, all others, 7, 8, 9, 10 and 11, are primary diamines. All these analogs were prepared by using the same general procedure without any problems. Their analytical HPLC profiles and ESI-MS spectra are found in figures 3.13 to 3.18 below. 


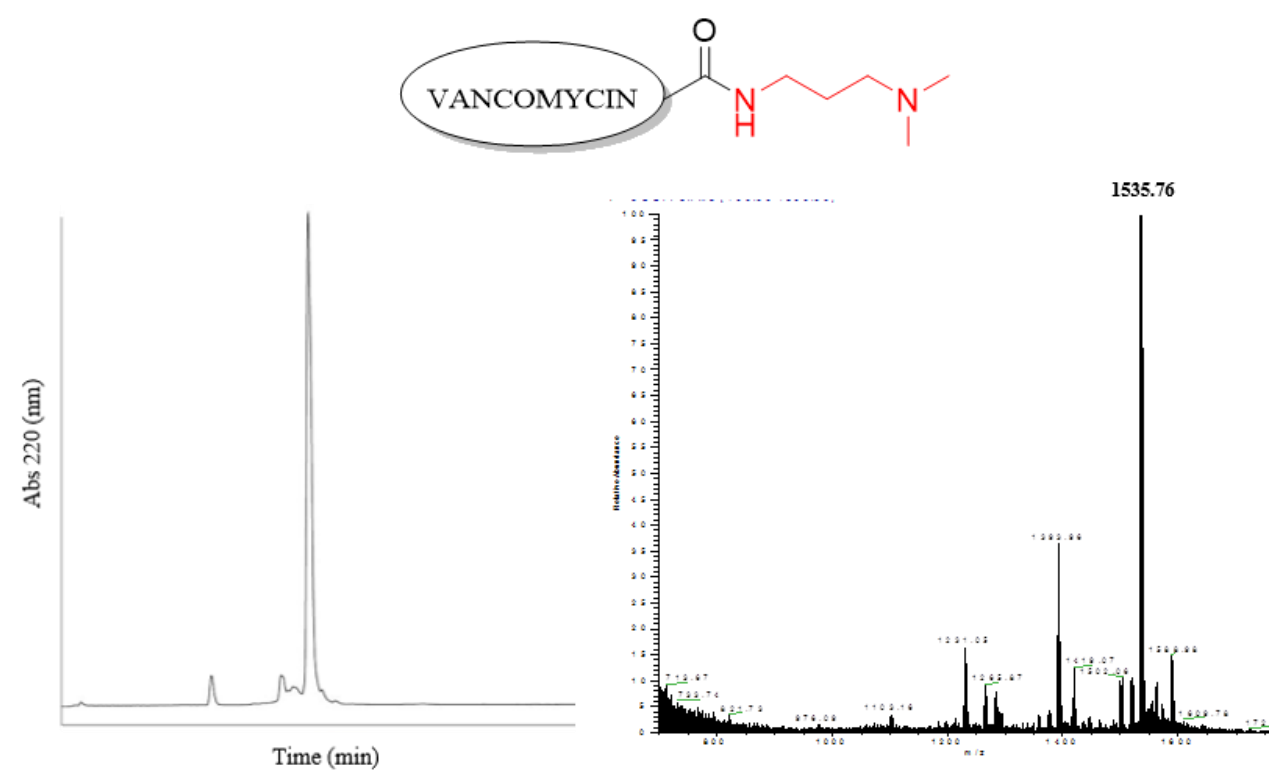

Figure 3.13 Analytical HPLC profile (left panel) and the ESI-MS (right panel) of Van-dimethylaminopropylamine 6. Observed $\left[\mathrm{M}+\mathrm{H}^{+}\right]^{+}=1535.76$.

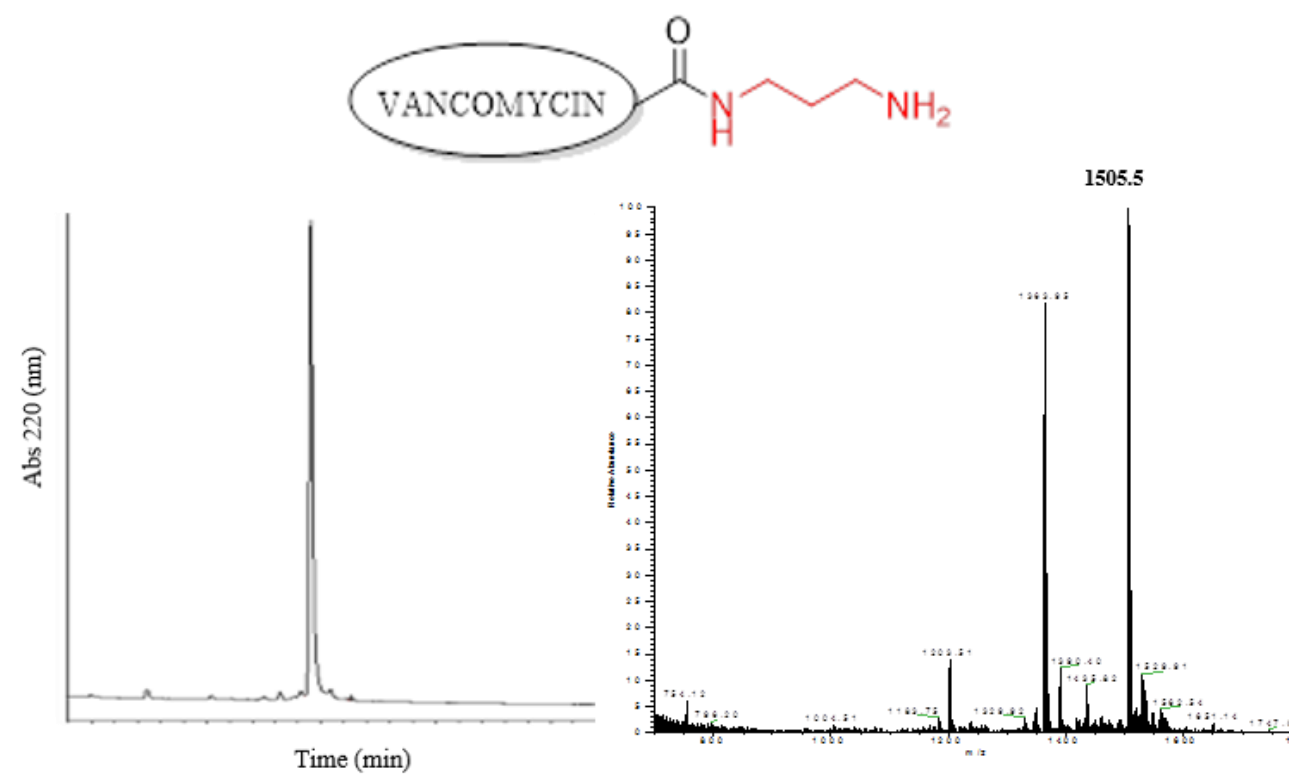

Figure 3.14 Analytical HPLC profile (left panel) and the ESI-MS (right panel) of Van-diaminopropane 7. Observed $\left[\mathrm{M}+\mathrm{H}^{+}\right]^{+}=1505.5$. 

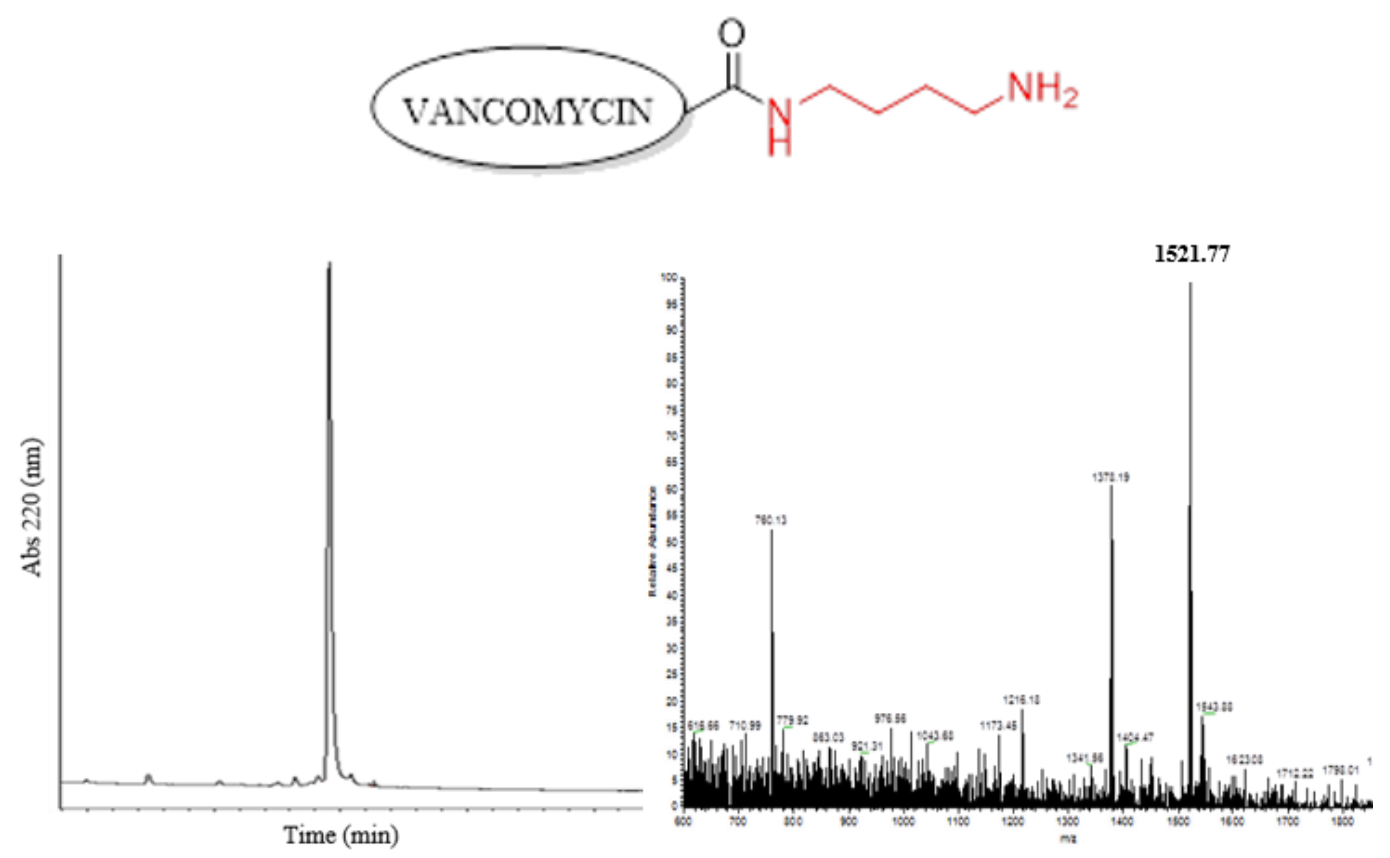

Figure 3.15 Analytical HPLC profile (left panel) and the ESI-MS (right panel) of Van-diaminobutane 8. Observed $\left[\mathrm{M}+\mathrm{H}^{+}\right]^{+}=1521.77$.
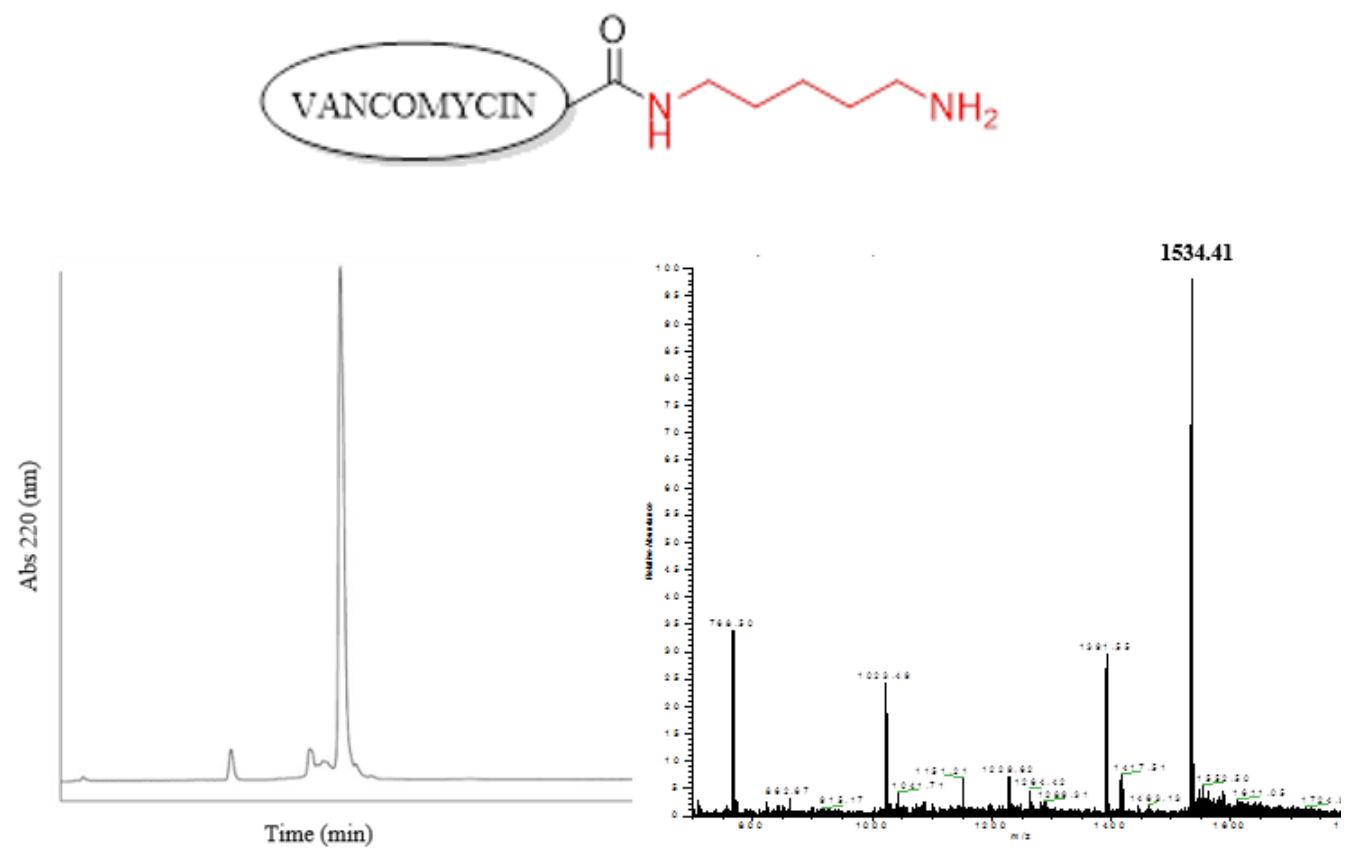

Figure 3.16 Analytical HPLC profile (left panel) and the ESI-MS (right panel) Van-diaminopentane 9. Observed $\left[\mathrm{M}+\mathrm{H}^{+}\right]^{+}=1534.41$. 


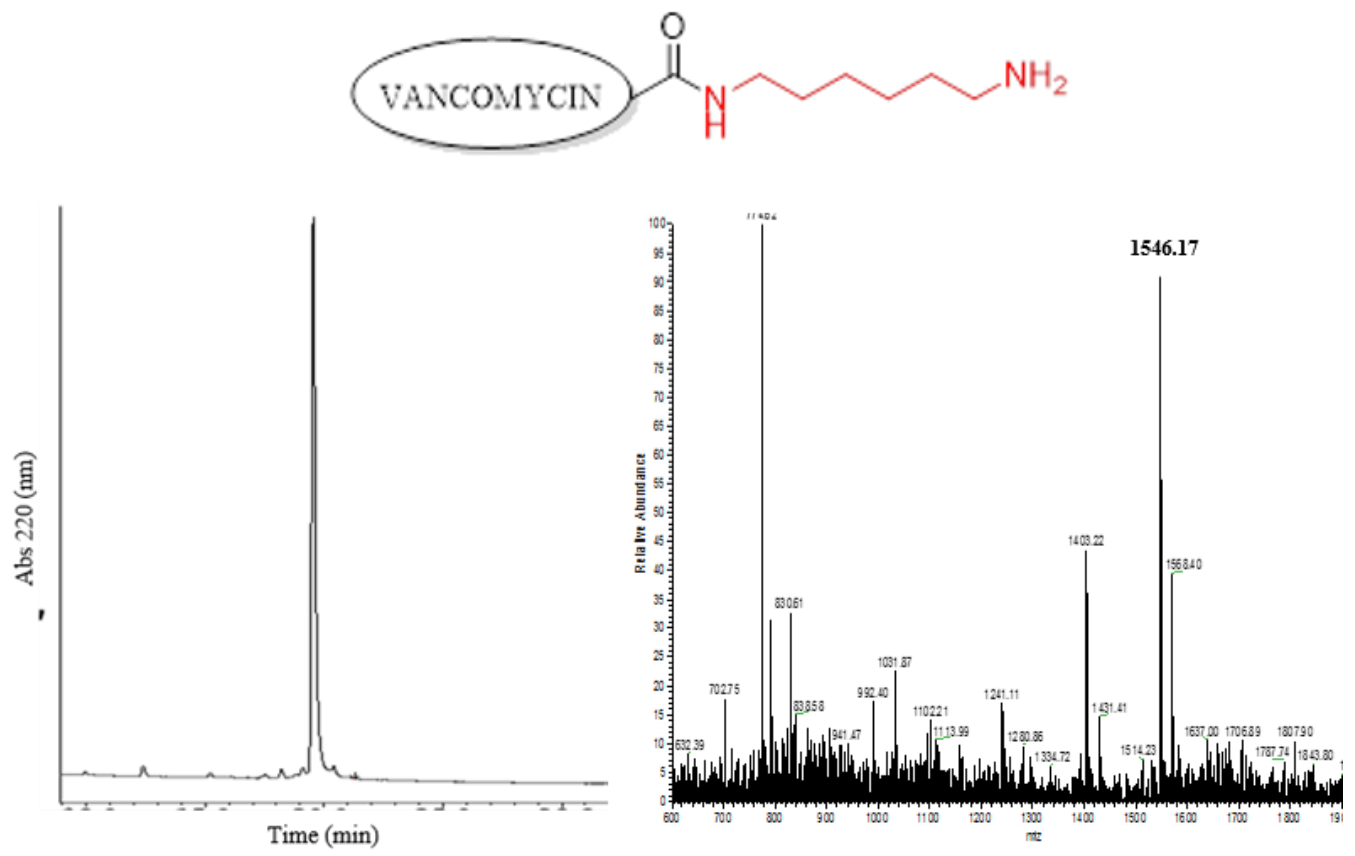

Figure 3.17 Analytical HPLC profile (left panel) and the ESI-MS (right panel) of Van-diaminohexane 10. Observed $\left[\mathrm{M}+\mathrm{H}^{+}\right]^{+}=1546.17$.

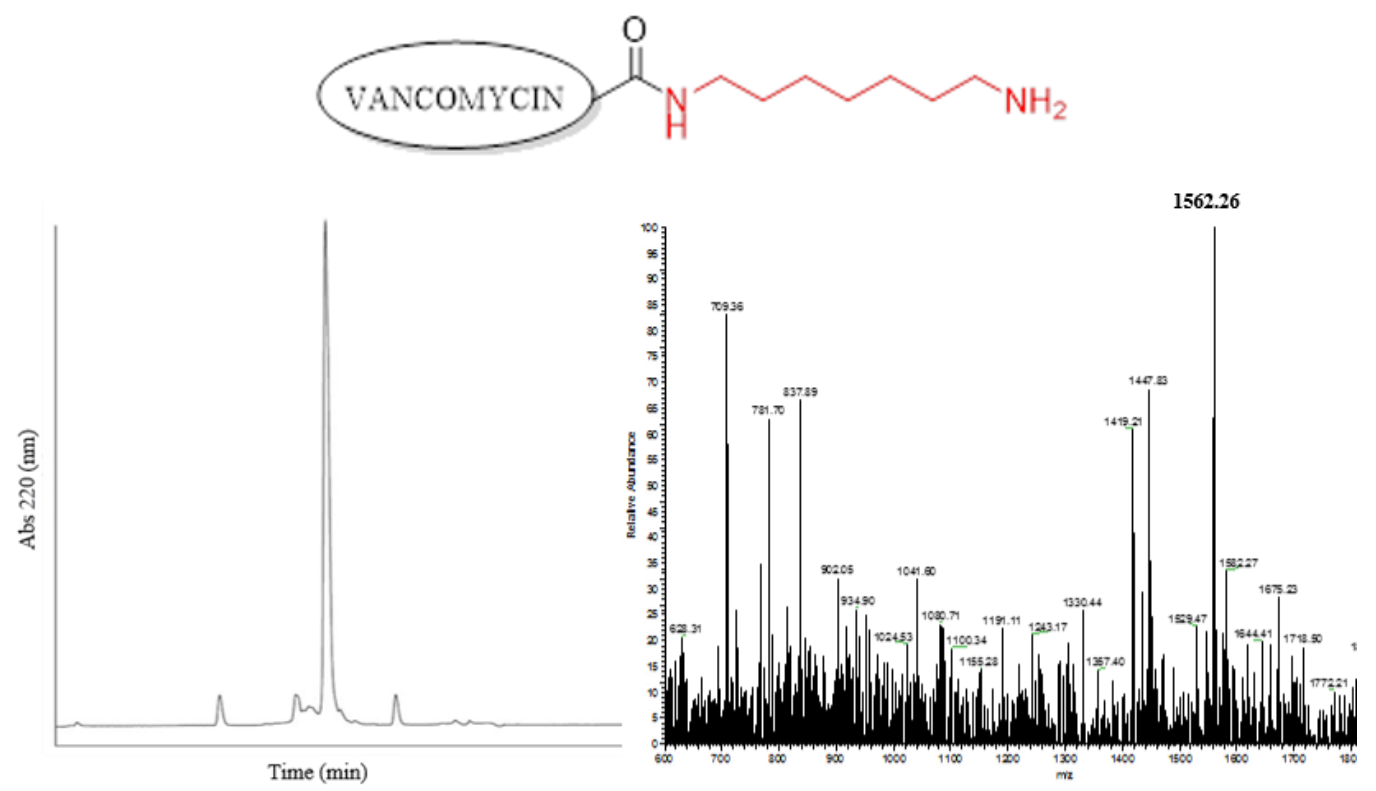

Figure 3.18 Analytical HPLC profile (left panel) and the ESI-MS (right panel) of Van-diaminoheptane 11. Observed at $\left[\mathrm{M}+\mathrm{H}^{+}\right]^{+}=1562.26$. 


\subsubsection{Synthesis of 12 (Van-O-phenylenediamine)}

The aliphatic amines were coupled with vancomycin to form different aliphatic amide analogs. The analogs formed by diamines attached with aromatic rings would reveal the importance of aromatic groups. The aromatic rings are planar and more rigid in conformation, and their pi-electronic cloud stack also makes them to have the ability to engage in electrostatic interactions with other positively charged groups. The comparison with linear aliphatic amide analogs will reveal interesting information.

Although this analog was synthesized using similar chemistry of amide bond formation, there was some difference in solubility and reactivity. O-phenylenediamine was not easily soluble in the solvent used. Ultrasonication was used to assist its solubilization. The product eluted out in HPLC at a time close to vancomycin. The HPLC profile and ESI of the required product are shown below.

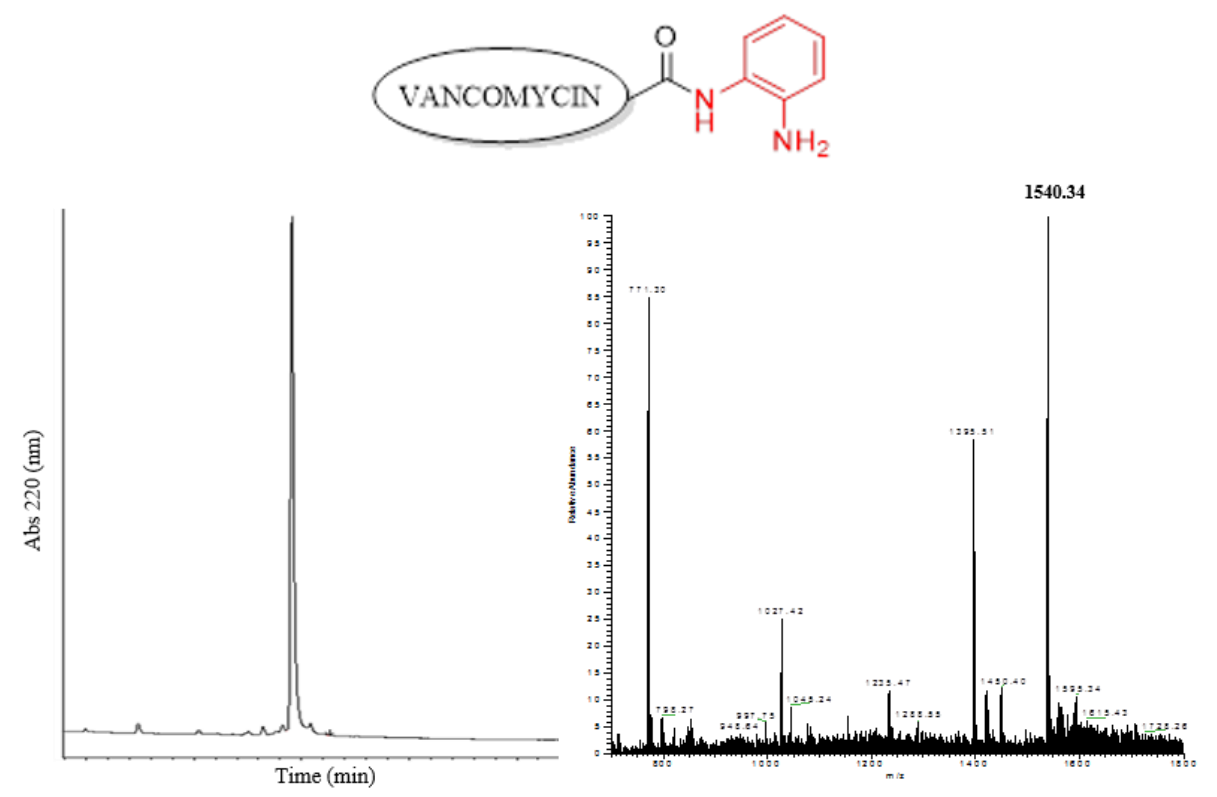

Figure 3.19 Analytical HPLC profile (left panel) and the ESI-MS (right panel) of Van-O-phenylenediamine 12. Observed $\left[\mathrm{M}+\mathrm{H}^{+}\right]^{+}=1540.34$. 


\subsubsection{Synthesis of 13 (Van-piperazine)}

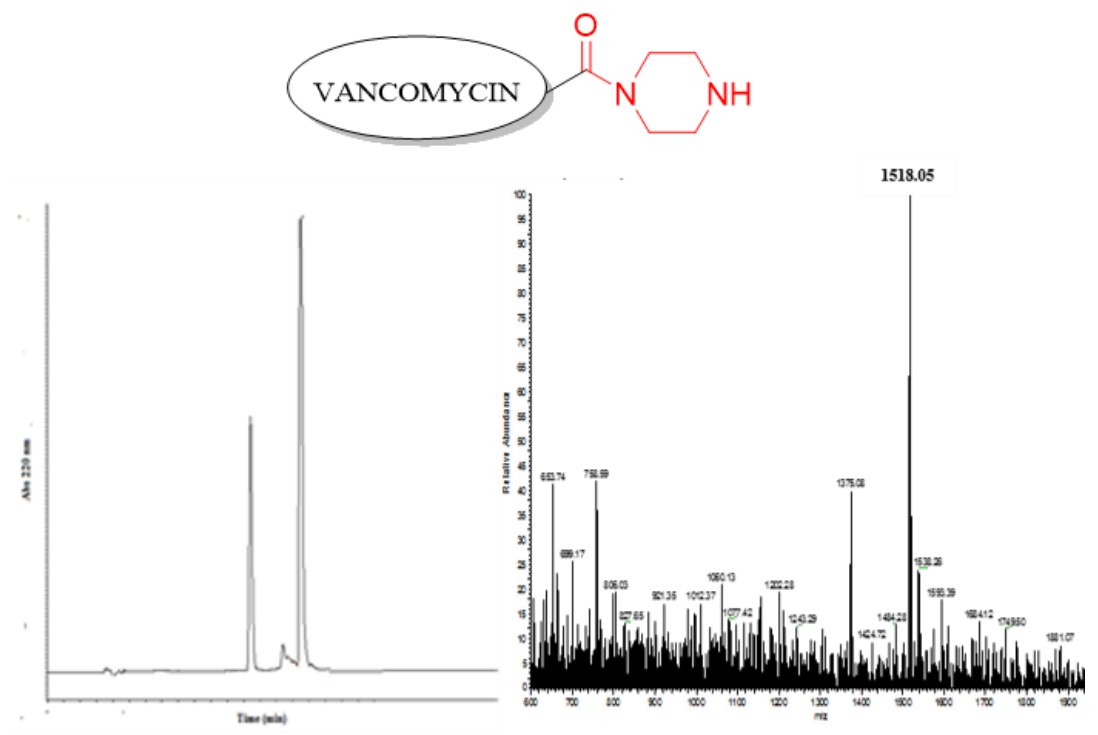

Figure 3.20 Analytical HPLC profile (left panel) and the ESI-MS (right panel) of Van-piperazine 13. Observed $\left[\mathrm{M}+\mathrm{H}^{+}\right]^{+}=1518.0$.

This analog with a non-aromatic cyclic ring may provide valuable information about its impact on the antibacterial activity. The cyclic diamine is more rigid than the linear diamines but less rigid than the aromatic one.

\subsubsection{Synthesis of 14 (Van-diethylenetriamine)}

To understand the impact of an extra amino group, a triamine, diethylenetriamine, was used to synthesize the analog. The triamine has the same number of atoms in its backbone as 1,5-diaminopentane. This provides an interesting comparison between these two analogs. Being sterically less hindered, the primary amines are much more likely to react than the secondary amine. So in this reaction, it is assumed that either one of two primary terminal amines would react with the carboxyl group of vancomycin. And only one product was formed. 


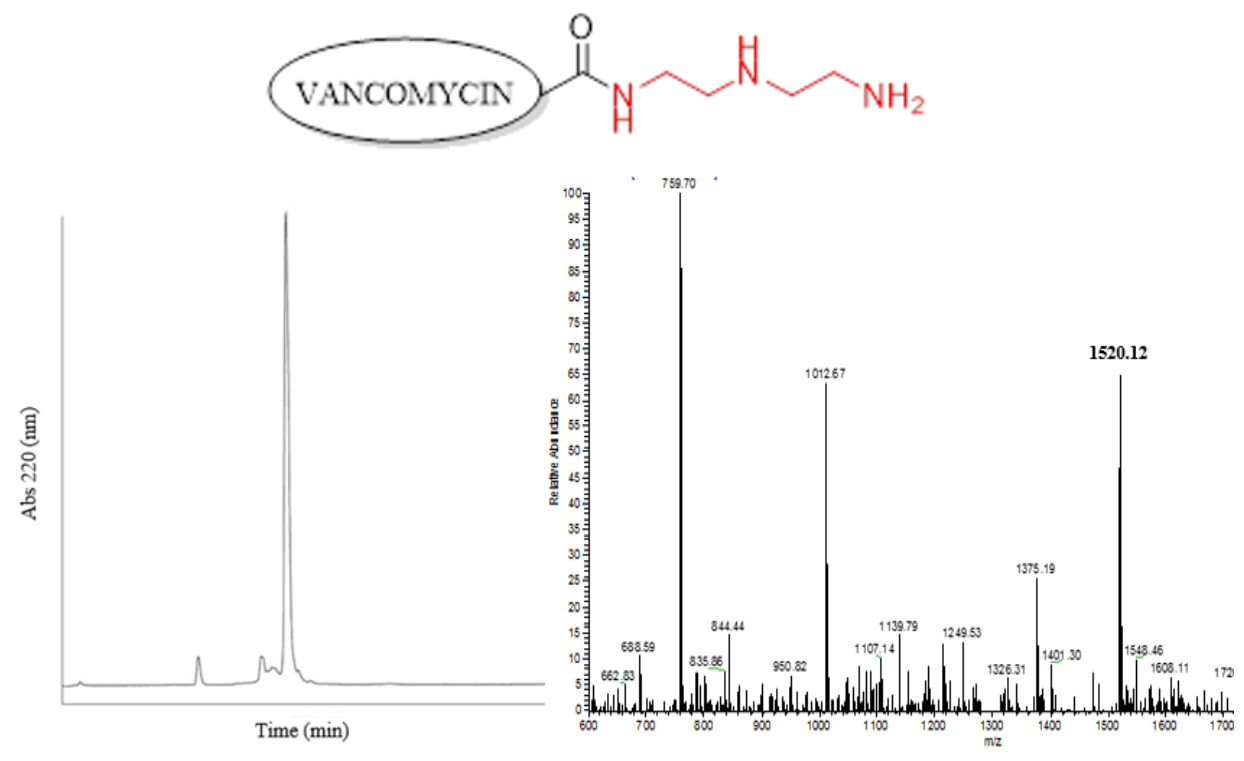

Figure 3.21 Analytical HPLC profile (left panel) and the ESI-MS (right panel) of the Van-diethylenetriamine 14. Observed $\left[\mathrm{M}+\mathrm{H}^{+}\right]^{+}=1520.12$.

\subsubsection{Antimicrobial studies of different analogs}

Radial diffusion assays were conducted to determine the antibacterial activities of the various analogs. In the study, both Gram positive and Gram negative strains were employed to determine the efficacy of these compounds. E.coli used in the study is the lab strain, and with its poor membrane permeability for the drugs it represents a stringent test for the efficacy of the new analogs. The assays can reveal whether the presence of additional free amines and a hydrocarbon chain of varying lengths at the C-terminal of vancomycin would improve the activity. It can help us understand the SAR between antibacterial effects and the increase in cationic charge and the chain length. It will also test the rationale that cationic hydrocarbon moieties attached through carboxamide formation can assist in bacterial membrane binding. Our rationale is that the presence of an ionizable amine may enhance the affinity towards negatively charged phospholipids and the hydrocarbon further facilitate attachment toward lipid groups. It is assumed that a free 
primary amine and a tertiary amino group with dimethyl substitutions both can exert their cationic effect towards the cell wall and therefore have a similar effect.

Table 3.1 The antibacterial activities of different carboxamide analogs of vancomycin.

\begin{tabular}{|c|c|c|c|c|}
\hline \multicolumn{5}{|c|}{ In vitro antimicrobial study of analogs $(\mathrm{MIC})(\boldsymbol{\mu M})$} \\
\hline & $\begin{array}{l}\text { Staphylococe } \\
\text { aureus }\end{array}$ & $\begin{array}{l}\text { Escherichia } \\
\text { Coli }\end{array}$ & $\begin{array}{l}\text { Bacillus } \\
\text { subtilis }\end{array}$ & $\begin{array}{l}\text { Pseudomonas } \\
\text { aeruginosa }\end{array}$ \\
\hline Vancomycin & $4.4 \pm 0.4$ & $1.7 \pm 0.3$ & $0.5 \pm 0.05$ & $20.2 \pm 3$ \\
\hline Van-cystamine (1) & $0.43 \pm 0.1$ & $0.13 \pm 0.05$ & $0.1 \pm 0.01$ & $1.3 \pm 0.4$ \\
\hline Van-cysteamine (2) & $2.0 \pm 0.3$ & $2.69 \pm 0.4$ & $2.8 \pm 0.4$ & $3.8 \pm 1$ \\
\hline Van-ethylenediamine (3) & $0.6 \pm 0.2$ & $0.4 \pm 0.06$ & $0.2 \pm 0.05$ & $2 \pm 0.4$ \\
\hline $\begin{array}{l}\text { Van-dimethylethylelene } \\
\text { diamine (4) }\end{array}$ & $0.4 \pm 0.1$ & $0.18 \pm 0.04$ & $0.6 \pm 0.1$ & $8.4 \pm 2$ \\
\hline $\begin{array}{l}\text { Van-trimethyl } \\
\text { Ethylenediamine (5) }\end{array}$ & $0.73 \pm 0.2$ & $0.85 \pm 0.1$ & $0.15 \pm 0.04$ & $5.3 \pm 1.3$ \\
\hline $\begin{array}{l}\text { Van-dimethylamino- } \\
\text { propylamine (6) }\end{array}$ & $0.52 \pm 0.1$ & $0.1 \pm 0.5$ & $0.1 \pm 0.04$ & $0.23 \pm 0.04$ \\
\hline Van-diaminopropane (7) & $0.22 \pm 0.05$ & $0.07 \pm 0.04$ & $0.07 \pm 0.02$ & $0.11 \pm 0.05$ \\
\hline Van-diaminobutane (8) & $0.13 \pm 0.05$ & $0.09 \pm 0.05$ & $0.02 \pm 0.01$ & $0.12 \pm 0.05$ \\
\hline Van-diaminopentane (9) & $0.1 \pm 0.05$ & $0.02 \pm 0.005$ & $0.02 \pm 0.01$ & $0.15 \pm 0.1$ \\
\hline Van- diaminohexane (10) & $0.11 \pm 0.05$ & $0.09 \pm 0.02$ & $0.02 \pm 0.01$ & $0.17 \pm 0.06$ \\
\hline Van-diaminoheptane (11) & $0.14 \pm 0.1$ & $0.11 \pm 0.5$ & $0.04 \pm 0.02$ & $0.15 \pm 0.05$ \\
\hline Van-O-phenylenediamine(12) & $0.52 \pm 0.1$ & $0.08 \pm 0.03$ & $0.08 \pm 0.03$ & $0.56 \pm 0.03$ \\
\hline Van-piperazine (13) & $1.1 \pm 0.3$ & $1.3 \pm 0.2$ & $1.68 \pm 0.2$ & $2.3 \pm 0.4$ \\
\hline Van-diethyltriamine (14) & $2.0 \pm 0.4$ & $0.69 \pm 0.1$ & $1.2 \pm 0.3$ & $0.49 \pm 0.1$ \\
\hline
\end{tabular}

As seen from the above table, many molecules show excellent properties, and some compounds are 20-50 folds more active than the parent compound. 
The studies commenced with the cystamine and cysteamine derivatives. 1 has a terminal amine whereas $\mathbf{2}$ has a thiol moiety instead. $\mathbf{1}$ shows more potent activity than $\mathbf{2}$, which suggests that an ionizable amine gives better activity than a non-ionizable thiol. 2 indeed has comparable activities with vancomycin.

$\mathbf{3}$ and its closely related analogs $\mathbf{4}$ and $\mathbf{5}$ have a similar ethylenediamine backbone. These analogs exhibited interesting results. All three analogs exhibited excellent and more potent antimicrobial activities than vancomycin. 3, 4, and $\mathbf{5}$ have comparable activity against Staphylococcus aureus, with $\mathbf{4}$ being a bit more active than $\mathbf{3}$ and $\mathbf{5}$. The same is true for E. coli. They have activities in almost same order of magnitude against Bacillus subtilis, with 4 that being slightly less active. The trend is similar for Pseudomonas aeroginosa with poorer MIC for 4 than other two compounds. Overall it reveals that the ethylamine moiety protruding from the carboxyl end of vancomycin is good for antibacterial activity, and the presence of a primary amine or tertiary amine as the terminal group does not significantly influence the activity of these analogs.

Compound 6-11 are prepared from diamines with increasingly longer carbon chains, and all the analogs have a terminal amine. Compound $\mathbf{6}$ and $\mathbf{7}$ are propyl-chain analogs where $\mathbf{6}$ have a tertiary amino group extending out while $\mathbf{7}$ has a primary amine at the same location. Compound $\mathbf{6}$ and 7 possess excellent activities in comparison to their parent compound vancomycin. Analog 7 is two-fold better than $\mathbf{6}$ against Staphylococcus aureus and Pseudomonas aeroginosa; both compounds have comparable activity against Escherichia coli and Bacillus subtilis. It can be seen that all six compounds have excellent activities against both Gram negative and Gram positive bacteria. Compounds 7-11 can inhibit Staphylococcus aureus with concentrations less than $0.24 \mu \mathrm{M}$. The highest improvement is 40 folds for 9. Compounds 6-11 also demonstrate exceptional activity against lab strains of E.coli and Bacillus subtitles. The analogs show improvement in efficacy up to 25 folds than parent compound for compound 8, 9 and 10. Overall, it confirms that as hydrocarbon chain is increased along with amino as a terminal group, the activity also improves. 
To better understand the impact of different diamines, cyclic amines were used to form carboxamide analogs with vancomycin. Analog 12 (Van-O-phenylenediamine) exhibits potent activities against both Gram-negative and positive strains. Its activity against all four strains is closer in magnitude to the activities of the linear diamine compounds 7-11. It has a more rigid conformation in its C-ter amide part of the structure than the linear diamine analogs. Still, it exhibits quite potent activity. Along with the aromatic ring, it also has a free amine which is less basic than the aliphatic amines. This indicates that if the analog possesses an amine and a hydrocarbon entity, the cationic and lipophilic properties synergize to make the analog quite potent. Linear moieties are not the only potential functional groups needed to enhance the activity of the analogs.

To understand the impact of cyclic non-aromatic analogs, $\mathbf{1 3}$ was synthesized with the appendage of piperazine at the carboxyl group of vancomycin. In comparison to $\mathbf{1 2}$, its activity dropped a few folds, which implies that a lack of protruding amine may negatively affect the antibacterial potential. It reveals that amino groups play an important in the activity of the compounds. Although its activity was lower than 12, still compound $\mathbf{1 3}$ exhibited good antibacterial potency. Its activity against Staphylococcus aureus was three folds better than vancomycin and comparable against E.coli. It shows better activity against P.aeroginosa with a MIC of $2.3 \mu \mathrm{M}$ but poorer activity against Bacillus subtitles than vancomycin.

The compound 14 contains two additional ionizable amino groups compared to vancomycin. Its activity lies within the therapeutic range and MICs are lower than the parent compound for Staphylococcus aureus, Escherichia coli, and Pseudomonas aeroginosa, although vancomycin shows better activity for Bacillus Subtilis. However, 14 is less potent than compounds 7-11 which represent a set of diamine-derived carboxyamides. A notable comparison is with analog 9 which has the same number of atoms in the backbone as 14. Compound $\mathbf{1 4}$ is 20 folds less potent than 9 for Staphylococcus aureus, two times less active against Pseudomonas aeruginosa and almost 
ten times lower in activity against E.coli and Bacillus subtilis. It seems that introducing an extra amine in the middle of the hydrocarbon backbone affects the activity unfavorably.

Most of the synthesized compounds possess a positively charged amine and a varying degree of lipophilic character based on its chain length and nature of the appended moiety. Yarlagadda et al. synthesized cationic lipophilic analogs by reacting different moieties on C-terminal of vancomycin. They have proposed that a cationic charge could better facilitate interaction of the compounds with the negatively charged bacterial membrane and the lipophilic moiety assist the compound in anchoring on the bacterial wall for a longer duration. ${ }^{76}$ Similarly, lipophilic carboxamide analogs of teicoplanin also show improved activity than the parent compound. These teicoplanin analogs also have hydrophobic cationic moieties attached at the C-ter carboxyl group.

In our studies, a potent antibacterial activity against Gram-negative Pseudomonas aeruginosa was also observed for some of the analogs, although more studies are needed to further confirm this finding. It has been shown previously that molecules with hydrophilic nature and a net positive charge may pass through the outer membrane of Gram-negative bacteria. The presence of porins on the outer membrane is believed to accommodate the penetration of positively charged entities. If similar mechanism applies to vancomycin analogs with terminal amine moieties, it may explain the potent activity of certain compounds against Pseudomonas aeroginosa. The compounds synthesized in our study have higher positive charge ratio due to the presence of a free amine. It is a possible reason for enhanced activities observed. However, to complete the study, antimicrobial testing on the analogs against vancomycin resistant strains will be more significant.

\subsubsection{Hemolysis}

Some of the vancomycin analogs have shown quite promising antibacterial activities. To assess their safety profiles, we conducted hemolytic assays on the analogs synthesized. Hemolysis studies were conducted using fresh erythrocytes of a human. The results show no significant hemolytic activity of vancomycin analogs. Triton X100 1\% was used as a 
positive control; the phosphate buffer solution was used as a negative control. The hemolysis observed with $1 \%$ Triton X-100 was considered as a reference value of $100 \%$ hemolysis. The analogs with different molar concentrations were tested with maximum concentration up to $100 \mu \mathrm{M}$. The results of hemolysis observed at $100 \mu \mathrm{M}$ of each drug are shown in the table. The safety profiles of all the analogs tested are comparable to the parent compound - vancomycin.

Table 3.2 The hemolytic activity of different vancomycin analogs.

The percentage of hemolysis at $100 \mu \mathrm{M}$ of different vancomycin derivatives

\begin{tabular}{l|l|l|l|l|l|l|l|l|l|l}
\hline Sample & Trx-1\% & Van & $\mathbf{2}$ & $\mathbf{3}$ & $\mathbf{6}$ & $\mathbf{7}$ & $\mathbf{8}$ & $\mathbf{9}$ & $\mathbf{1 1}$ & $\mathbf{1 2}$ \\
\hline Hemolysis & $100 \%$ & $1.69 \%$ & $2.25 \%$ & $0.51 \%$ & $1.34 \%$ & $1.57 \%$ & $1.77 \%$ & $1.85 \%$ & $1.85 \%$ & $1.77 \%$ \\
\hline
\end{tabular}

Trx-1\% refers to $1 \%$ TritonX-100. 2 (Van-cysteamine), 3 (Van-ethylenediamine), 6 (Van-

3-dimethyl-amino-1 propylamine), 7 (Van 1,3 diamino propane), 8 (Van 1,4 diamino butane), 9 (Van 1,5 diamino pentane), 11 (Van 1,7 diamino heptane), 12 (Van-O phenylenediamine).

\subsection{Conclusion}

In this study, a previously underexplored strategy to make vancomycin analogs is utilized. A simple single-step synthetic procedure is developed that couples diamines with the carbonyl group of vancomycin. Using this method, fourteen analogs have been synthesized. Most the synthesized analogs exhibit excellent antibacterial profiles. Almost all of them are more active than the parent drug of vancomycin. The strategy is not only a simple method to develop vancomycin analogs, but it also offers a very convenient strategy to conjugate other antibacterial entities with vancomycin to create hybrid antibiotics which should have synergistic effects and impart dual mechanism of action.

Some of the vancomycin analogs synthesized are many folds more potent than vancomycin. The rationale behind increased activity is probably due to the presence of the 
free amine at the C-terminal end. Amines may enhance the affinity towards negatively charged phospholipids and the hydrocarbon further facilities attachment toward lipids. The studies show that the analogs with a linear aliphatic terminal amine have very potent antibacterial activities. However, the piperazine analog has lower antibacterial activities than these analogs. It suggests that a lack of protruding amine may impact the antibacterial potential. The strategy presented in this chapter is quite promising, and it paves the pathway to develop many more active compounds. In the future, extensive antibacterial studies in in-vitro and in-vivo systems using vancomycin-resistant strains will be needed. It will help to narrow down the leads that can be considered for the next stage of drug development.

\subsection{Materials and methods}

\subsubsection{Material and Instruments}

Vancomycin hydrochloride was from Sigma-Aldrich; 1,5-diaminopentane, diethylamine, benzylamine, ethylenediamine, 4-chlorobiphenyl-4-carbaldehyde, 3dimethylamino-1-propylamine, diethylenetriamine were from Alfa Aesar; 1,3diaminopropane; 1, 4-diaminobutane were from Alfa Aesar; 1,6-diaminobutane was from Alfa Aesar; 1,7-diaminoheptane was from Lancaster; Piperazine was from Aeros organics; N, N-Dimethylethylenediamine was from Sigma-Aldrich; PyBOP was from GL Biochem (Shanghai) Ltd; TSB was from Fluka. Mueller Hinton Broth (MHB) and agarose were from Vivantis; Tryptone was from BD Bacto ${ }^{\mathrm{TM}}$; Yeast extract from BD Bacto ${ }^{\mathrm{TM}}$; Sodium chloride from Merck KGaA. O-phenylenediamine was purchased from Alfa Aesar.

All the instruments used for HPLC and mass spectrometry analyses in this chapter are the same as described in Chapter 2. 


\subsubsection{Methods}

\subsubsection{Synthesis of Van-cystamine (1)}

$1 \mu \mathrm{mol}$ of pre-lyophilized vancomycin was dissolved in $1 \mathrm{~mL}$ of DMF, followed by addition of $200 \mu \mathrm{L}$ 0.1M Cystamine dihydrochloride $(20 \mu \mathrm{mol})$. Then $10.2 \mu \mathrm{L}$ of DIEA (60 $\mu \mathrm{mol})$ was added, followed by $50 \mu \mathrm{L}$ of $0.1 \mathrm{M}$ PyBOP $(5 \mu \mathrm{mol})$. The reaction mixture was vortexed and incubated at room temperature for $2 \mathrm{~h}$. Then it was lyophilized for an overnight before it was dissolved in $1 \mathrm{~mL}$ of $\mathrm{dd} \mathrm{H}_{2} \mathrm{O}$. The mixture was analyzed first with analytical RP-HPLC and then purified by semi-preparative RP-HPLC. The yield was about $42 \%$

\subsubsection{Synthesis of Van-cysteamine (2)}

$1 \mu \mathrm{mol}$ of pre-lyophilized vancomycin was dissolved in $1 \mathrm{~mL}$ of DMF, followed by addition of $20 \mu \mathrm{L}$ of $1 \mathrm{M}$ Cysteamine hydrochloride $(20 \mu \mathrm{mol})$. Then $10.2 \mu \mathrm{L}$ of DIEA (60 $\mu \mathrm{mol})$ was added, followed by $50 \mu \mathrm{L}$ of $0.1 \mathrm{M}$ PyBOP $(5 \mu \mathrm{mol})$. The reaction mixture was vortexed and incubated at room temperature for $2 \mathrm{~h}$. After incubation, the tube was frozen with liquid nitrogen and lyophilized for an overnight before it was dissolved in $1 \mathrm{~mL}$ of $\mathrm{ddH}_{2} \mathrm{O}$. The mixture was analyzed first with analytical RP-HPLC and then purified by semi-preparative RP-HPLC. The yield was about $25 \%$.

Reduction of Van-cystamine to Vac-cysteamine: Dried Van-cystamine was dissolved in $500 \mu \mathrm{L}$ of $\mathrm{ddH}_{2} \mathrm{O}$ and $40 \mu \mathrm{L}$ of $1 \mathrm{M}$ TCEP and was incubated at room temperature for $1 \mathrm{~h}$.

\subsubsection{Synthesis of Van-diamine derivatives $(3,4,5,6,7,8,9,10,11$ and} 13)

$14.8 \mathrm{mg}$ of vancomycin hydrochloride $(0.01 \mathrm{mmol})$ was solubilised in $150 \mu \mathrm{DMF}$ and $150 \mu \mathrm{DMSO}$. The mixture was vortexed and placed in an ultrasonicator until it was completely dissolved. An excess amount of the diamine $(0.2-0.5 \mathrm{mmol})$ was added to the 
dissolved vancomycin hydrochloride. In addition to being a reagent, the diamine also served as a base. $10.4 \mathrm{mg}$ of PyBOP (0.02 mmol) was dissolved in $100 \mathrm{ml}$ DMF/DMSO (1:1). The dissolved PyBOP was added to the reaction mixture. The reaction mixture was vortexed and incubated at room temperature for up to 90 minutes. The reaction mixture was quenched with $\mathrm{HCl}$ solution and lyophilized. The residue was purified by HPLC. The yields of these amide derivatives were $30-40 \%$.

\subsubsection{Van-O-phenylenediamine Analog (12)}

$21.6 \mathrm{mg}$ of O-phenylenediamine $(0.2 \mathrm{mmol})$ was added to $14.8 \mathrm{mg}$ of vancomycin hydrochloride $(0.01 \mathrm{mmol}) .300 \mu \mathrm{L}$ of DMF was added and the mixture was vortexed and placed in an ultrasonicator to solubilize the reactants. The remaining reactants were added after most of the O-phenylenediamine was dissolved. $10.4 \mathrm{mg}$ of PyBOP (0.02 mmol) was dissolved in $100 \mu \mathrm{L}$ of DMF. The dissolved PyBOP was added to the reaction mixture. 10uL of DIEA $(0.06 \mathrm{mmol})$ was then added to make up the reaction mixture. The reaction mixture was vortexed and incubated at room temperature for 90 minutes. The reaction mixture was quenched with $\mathrm{HCl}$ solution and lyophilized. The residue was purified by HPLC. The yield was about $30 \%$

\subsubsection{Hemolysis assay}

Hemolytic activities were determined by the lysis of fresh human erythrocytes. Fresh type B+ blood was donated by the author of the thesis. Erythrocytes from the blood $(1 \mathrm{ml})$ were collected by centrifuging at $700 \mathrm{rpm}$ for $15 \mathrm{~min}$. The pellet was then washed with PBS (1ml) five times and centrifuged at $1000 \mathrm{rpm}$ for $5 \mathrm{~min}$. The centrifuged erythrocytes were diluted 100X in PBS to obtain the stock dispersion. Peptide samples were prepared in two-fold dilutions in PBS and added to the stock dispersion in a 1:1 ratio in a $96-w e l l$ plate and incubated for $4 \mathrm{~h}$ at $37^{\circ} \mathrm{C}$ with gentle shaking. The concentrations of each peptide were $100 \mu \mathrm{M}, 75 \mu \mathrm{M}, 50 \mu \mathrm{M}, 25 \mu \mathrm{M}, 2.5 \mu \mathrm{M}, 0.5 \mu \mathrm{M}$. An equal volume of $1 \%$ Triton-X100 and PBS were used as positive and negative controls, respectively. 
When the sample causes hemolysis of erythrocytes, hemoglobin is released into the supernatant. After incubation of the sample and erythrocyte suspension, the plate was centrifuged at $1000 \mathrm{rpm}$ for $5 \mathrm{~min}$, and the supernatants were transferred to a new plate. Absorbance was read at 415 nm in an Infinite@ 200 PRO Tecan microplate reader. ${ }^{91}$ The percentage of hemolysis was calculated using the following method:

Percentage of hemolysis $=($ OD peptide - OD buffer $) /($ OD Triton-X - OD buffer $) \times 100$ 


\section{CHAPTER 4 SYNTHESIS OF TEIXOBACTIN ANALOGS}

\subsection{Introduction}

In our natural environment microbes are present in abundance. Soil sustains an immense diversity of microbes which, to a large extent, remain unexplored. Some known microbes have contributed significantly to the discovery of antibiotics. Most of the antibiotics used today are isolated from microbes of soil, including penicillin and those discovered up to the 1960s. After the 1960s, the prospect of finding new antimicrobial compounds from soil microbes has been extremely low due to limitations of the technology.

Recently, several research groups have made progress in improving the technology of growing previously uncultured microbes. The contribution of Kaeberlien et al. and his team is significant in this regard. They developed various techniques to grow "uncultivable microbes" in an environment that resemble their natural habitat. ${ }^{92}$ His team successfully developed a technology for the high-throughput cultivation of different species in tiny chambers known as iChip. With this technique, the soil sample is sufficiently diluted with liquid agar and delivered to the device as each chamber will get approximately one bacterial cell per compartment. A chip is designed to have about 400 small chambers. ${ }^{93}$ The device is then covered with two semipermeable membranes and then lowered down to its natural habitat. Bacterial cells in diffusion chambers interact with their environment through semipermeable membranes, which allows diffusion of growth factors, nutrients, and waste. ${ }^{94}$ The sophisticated technology increased the growth recovery of uncultured bacteria to 50 folds. Interestingly, the diffusion chambers provide better colony count than synthetic media, and species grown here are highly novel ${ }^{95}$. This technology made it possible to isolate and culture bacteria from the different habitats. It has given us the access to much greater molecular diversity than previously imagined. It leads the way to scavenge the natural sources for the 
antimicrobial metabolites. It opened another dimension towards drug discovery. It is reviving the Waksman approach for cultivating and screening microbes from soil or other sources.

Ling et al. reported the discovery teixobactin by using the iChip technology. ${ }^{23}$ This discovery was a tedious process, more than 10,000 strains were screened, and twenty-five hits were found. The most promising strain was discovered in a field in Maine, U.S.A. The Gramnegative bacterium that produces teixobactin was classified into genus Aquabacteria after genomic sequence. ${ }^{23}$ The discovery of teixobactin is regarded as a breakthrough in the field of infectious diseases. Since last thirty years, no compound was found to be as effective as teixobactin. It targets multiple sites in the bacterial cell wall, and it has revived the possibility of creating antibiotics which can resist bacterial resistance. ${ }^{96}$ Although its antibacterial spectrum is restricted towards Gram-positive bacteria, nevertheless potent and novel compounds are the need of present time against evolving Gram-positive species. ${ }^{96}$

\subsubsection{Teixobactin}

Teixobactin is a unique antimicrobial peptide comprising of 11 amino acid residues. It contains a cyclized 4-residue depsipeptide in its $\mathrm{C}$-terminal part and an $\mathrm{N}$-amine-methylated linear chain in its N-terminal part. ${ }^{23,97}$ It consists of five unnatural amino acid residues, four D-amino acids, i.e., D-Threonine, D-allo-Isoleucine, D-glutamine, N-methyl-Dphenylalanine and an unusual L-allo-enduracididine. The novelty of this antibiotic lies in a macrocyclic depsipeptide containing the uncommon amino acid of enduracididine. Its linear chain has methylated D-Phenylalanine as the first amino acid. It is a non-ribosomal peptide. The genomic sequencing helped to elucidate a gene cluster which is responsible for its biosynthesis.

Teixobactin resembles the class of peptides known as cyclodepsipeptides or "head to side chain cyclic peptides ${ }^{.98}$ In these cyclic polypeptides, the side chain hydroxyl group of a residue forms an ester bond with the carboxyl group of the C-terminal amino acid. Cyclodepsipeptides provide the valuable source of potent therapeutics moieties. Some are reported to have cytotoxic, antiviral and antimicrobial activities. ${ }^{98}$ Kahalalide $\mathrm{F}$ isolated from 
Elysia rufescens and green alga Bryopsis species exhibits potent anticancer activity and is under phase II clinical trial for prostate cancer. ${ }^{99}$ Daptomycin is another well-known example of cyclodepsipeptide, extracted from Streptomyces roseoporus. ${ }^{100}$ In 2003, FDA approved daptomycin for treatment of complicated skin infections caused by Gram-positive bacteria. ${ }^{101}$

Teixobactin is reported to be extremely effective against a broad range of pathogenic and drug-resistant bacteria. The in-vitro studies exhibited its antibacterial activity against Enterococci and M.tuberculosis with a MIC lower than $1 \mu \mathrm{g} / \mathrm{ml}$. Clostridium difficae and Bacillus anthracis are susceptible to teixobactin in the nanomolar range. ${ }^{23}$ Its activity against different species of Staphylococcus aureus underscores its importance. It can efficiently kill intermediate and vancomycin-resistant Staphylococcus aureus. Therefore it is considered to be a solution to the problem caused by the emerging superbugs (bacteria resistant to most available antibiotics), e. g., MRSA. ${ }^{102}$ Teixobactin has shown better antibacterial activity against Staphylococcus species than linezolid, daptomycin, and vancomycin.

The highly potent activity of teixobactin against Mycobacterium tuberculosis (Mtb) is a significant breakthrough in the global effort against tuberculosis (TB). Mtb has developed resistance to many known antibiotics used for its therapy. ${ }^{9-10}$ In current therapeutic regimens, multiple antibiotics are prescribed to treat $\mathrm{TB}$, but still the bacteria manage to compromise the anti-TB treatment consistently. ${ }^{12}$ Therefore, antimicrobial agents which can kill such rapidly evolving bacteria are a dire need of our time. Teixobactin may serve as a potential treatment for $\mathrm{TB}$, as it exhibited an antibacterial activity of $0.125 \mu \mathrm{g} / \mathrm{ml}$ against virulent (H37Rv) drugresistant isolate of Mtb. It has a potential to change the therapy regimen of TB from multiple antibiotics into a single drug. Teixobactin is mostly active against Gram-positive species except for certain E.coli strains. ${ }^{23}$

\subsubsection{Evasion of drug resistance}

Ling et al. 23 tried to induce teixobactin resistance. Staphylococcus aureus is considered as an evolving superbug, and M.tuberculosis is reported to have MDR (multi-drugresistant) strain in more than 48 countries. ${ }^{13}$ Ling et al. exposed S.aureus and M.tuberculosis 
to sub-lethal doses of teixobactin. Serial passages of bacteria and antimicrobial agents were incubated together for 27 days. Even after such a long duration, no mutant resistant strain was observed. These results are very encouraging as it provides the evidence that certain compounds can delay or prevent the development of bacterial resistance. It can give us an alternative strategy of developing compounds which can be employed to combat the problem of emerging resistance. Teixobactin was also tested for its toxicity against mammalian cell lines of mouse embryo fibroblast NIH/3tc and human liver cancer cell lines HepG2 and was found safe with no toxicity. It is stable in blood and showed no hemolytic activity. Regarding killing kinetics, it killed S.aureus in a shorter time than vancomycin. So far it has shown no hERG inhibition genotoxicity, good plasma half-life and overall decent pharmacokinetic and pharmacodynamic parameters. Rodent infections were tested to determine its in vivo efficacy. A MRSA infection model showed activity at $0.5 \mathrm{mg} / \mathrm{kg}$, while immune-compromised mice with thigh infection model need $2.5-5 \mathrm{mg} / \mathrm{kg}$ to control infection. Vancomycin was used as positive control in these studies, and it achieved the same results with slightly higher doses. They also conducted the analysis for efficacy in Streptococcus pneumonia lung infection model while using amoxicillin as a positive control. ${ }^{23}$

The ability of teixobactin to prevent bacteria resistance after numerous generations advocate a mechanism that targets a non-protein target site, something which bacteria cannot alter readily. Its molecular weight of $1242 \mathrm{~g} / \mathrm{mol}$ includes it in relatively higher mol weight entities. Other closely related known antibiotics include vancomycin with a molecular weight of $1450 \mathrm{~g} / \mathrm{mol}$ and daptomycin another cyclodepsipeptide with $1619.7 \mathrm{~g} / \mathrm{mol}$ which target lipid substrate and interfere in cell wall biosynthesis.

\subsubsection{Mechanism of action}

Teixobactin is known to act on at least two molecules, Lipid II and Lipid III, with lipid II being suggested as the main target. Lipid II comprises of polyprene membrane anchors, GlcNAc, MurNAc and a linear pentapeptide chain. Lipid II is synthesized on "carrier lipid" within the cell and then flipped outside to transport these precursors for the manufacture of 
peptidoglycan wall. When bacteria need to construct the peptidoglycan layer, this disaccharide lipid II units polymerize, followed by cross-linking of the peptide chains. The progress of this biosynthesis is regulated by lipid II concentration. Teixobactin blocks Lipid II by forming a complex of teixobactin/lipid (2:1). ${ }^{96}$ Lipid III is a precursor of cell wall teichoic acid synthesis. It is needed to prevent degradation of existing peptidoglycan layer on the cell wall. When teixobactin binds with lipid III, it expedites lysis of cell wall.

Lipid II is also the target of vancomycin. It took three decades for vancomycin resistance to emerge because it is very difficult for bacteria to change the highly conserved Lipid II dipeptide structure of the peptidoglycan. ${ }^{103}$ The binding motif of teixobactin is thought to be the pyrophosphate sugar moiety of lipid II which is even less likely to change. It is assumed that it will take even longer for teixobactin resistance to appear. As teixobactin is a very promising candidate in the management of bacterial infectious diseases, it is an excellent model compound to make new derivatives which can form a new class of potent antibacterial agents in the fight against drug resistance.

\subsubsection{Reported syntheses of teixobactin analogs}

The aim of the project is the chemical synthesis of teixobactin analogs using a simple and efficient scheme. There are several recently published reports about the total synthesis of teixobactin and its analogs. As enduracidine is not commercially available, arginine is often used as a replacement of the synthetically challenging L-allo-enduracididine. So far, the synthesis of teixobactin and its analogs are achieved by solid phase peptide synthesis of the linear precursor, followed by solution-phase macrolactamization to get the final product. The schemes employed by different groups for teixobactin analog synthesis are shown below.

Jad et al. ${ }^{43}$ used 2-chlorotrityl resin for solid phase synthesis using alanine as a first amino acid at C-terminal (Figure 4.2). They then coupled D-threonine with unprotected alcohol side chain and added two more residues to form linear tetrapeptide before esterification. They used Alloc-protected Ile-OH for esterification as peptide on the linear chain has Fmoc protection. Esterification is a crucial step for the synthesis, and incomplete 
esterification can significantly reduce the yield of the final product. After esterification of isoleucine, they added Alloc-protected-Arg-OH on the esterified isoleucine. Then Fmoc-Ile$\mathrm{OH}$ from the linear chain was deprotected, and linear chain sequence was elongated till completion. Then the peptide was cleaved in the protected form using a special cleavage technique. The protected linear peptide was undergone cyclization between the C-ter carboxyl of Ala and the $N^{\alpha}$-amine of Arg. Then the reaction mixture was exposed to a conventional TFA deprotection/cleavage solution to remove all protecting groups. The final yield after purification and lyophilization was $6 \%$ from the crude linear peptide.

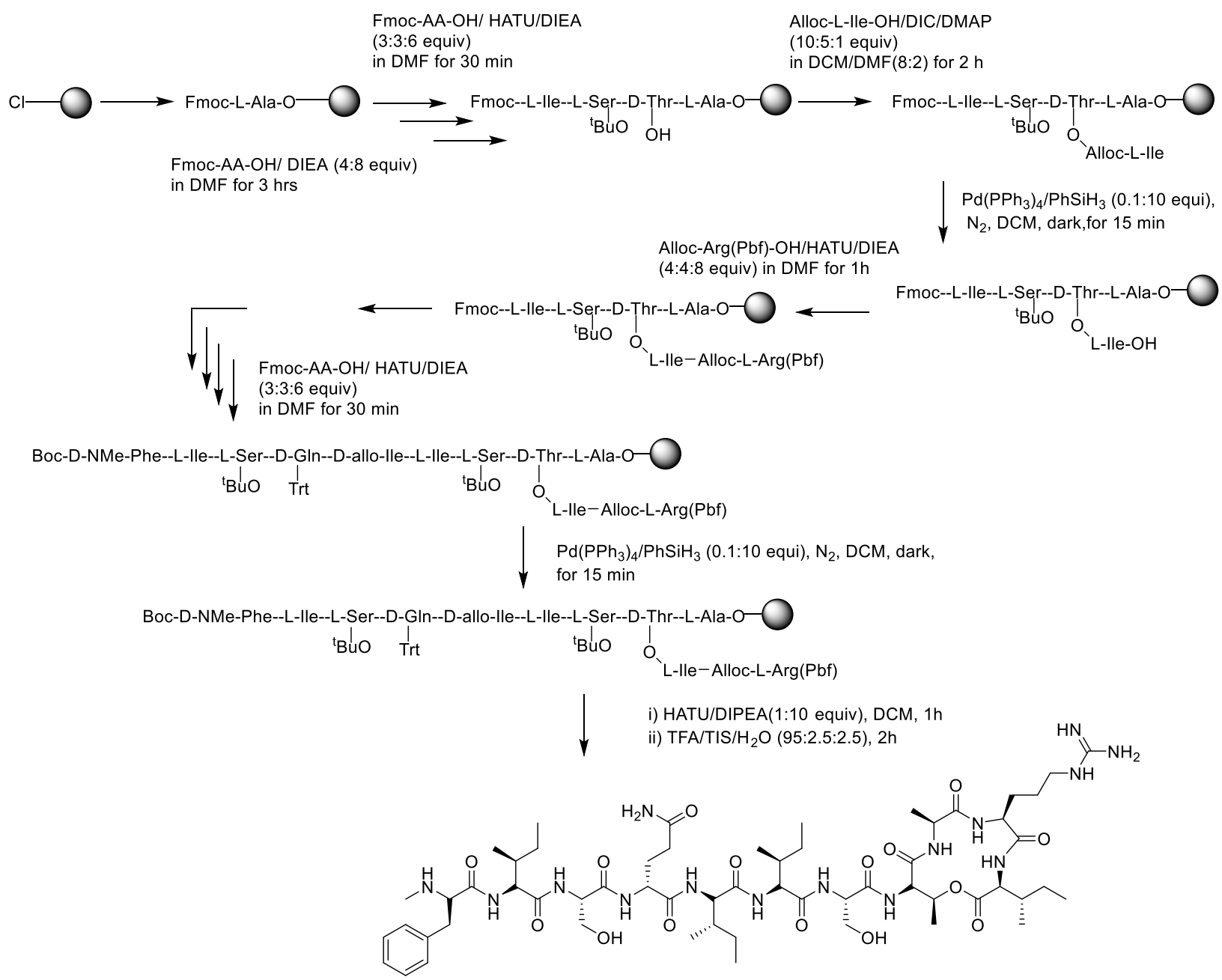

Figure 4.4.1 The scheme to synthesize teixobactin analog using solid phase peptide strategy employed by Jad et al. $^{43}$ 


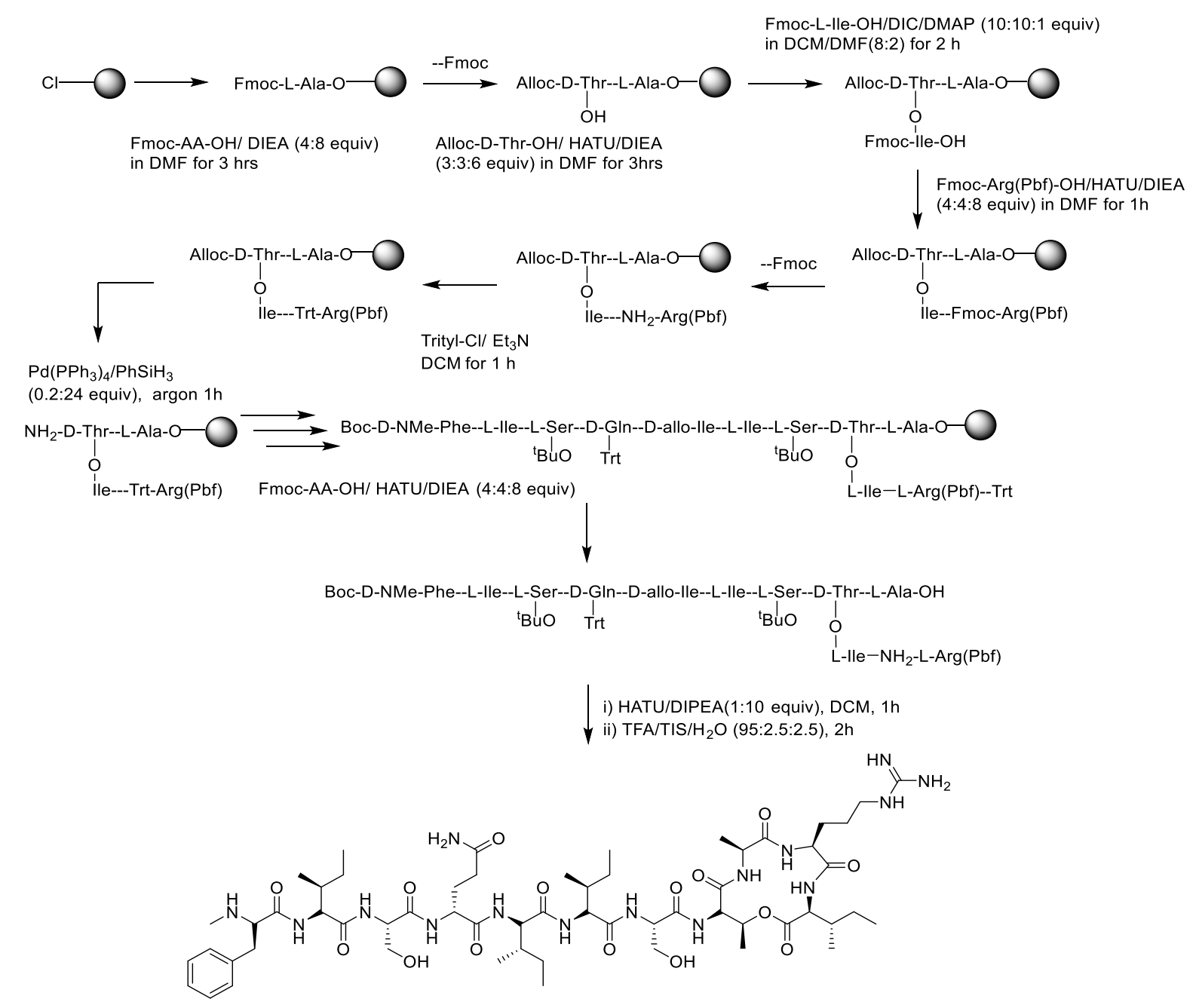

Figure 4.4.2. Solid phase employed by Parmer et al. to synthesize teixobactin analog. ${ }^{103}$

Parmer et al. published a strategy to synthesize teixobactin analog with a higher yield than the first reported method. ${ }^{104}$ They also used arginine as the L-allo enduracidine substitute. Figure 4.3 shows their method, which has some similarities with the first reported work of Jad et al. Parmer et al. utilized 2-chlorotriyl resin and alanine was the first amino acid coupled at the C-terminal. Then Alloc-protected D-threonine with free hydroxyl side chain was subsequently coupled. Then the crucial step of esterification with Fmoc-Ile-OH was conducted. Arginine was coupled and after Fmoc deprotection, N-terminal of Arginine was protected with the trityl group. The Alloc protection on the amine of D-threonine was 
deprotected, and the linear chain synthesis was completed with Boc-NMe-D-Phenylalanine as the final amino acid. The peptide was cleaved from the resin with $2 \%$ TFA, with the protecting groups still attached. Then the protected linear peptide obtained (in oil form) underwent insolution macrolactamization in the presence of 1 equivalent HATU and 10 equivalents DIEA for $1 \mathrm{~h}$. Cyclization was monitored with HPLC and once starting material was consumed, total deprotection with cleavage cocktail yield the final product. The synthesis is quite efficient as reported yield after purification and lyophilization is $22 \%$. They used a similar method in synthesizing analogs with the same sequence but without any D-amino acids. However, the antimicrobial activity of analog without any D-amino acid was lower than the analog with the D-amino acids in place. In all analogs, arginine is used in place of L-allo enduracidine.

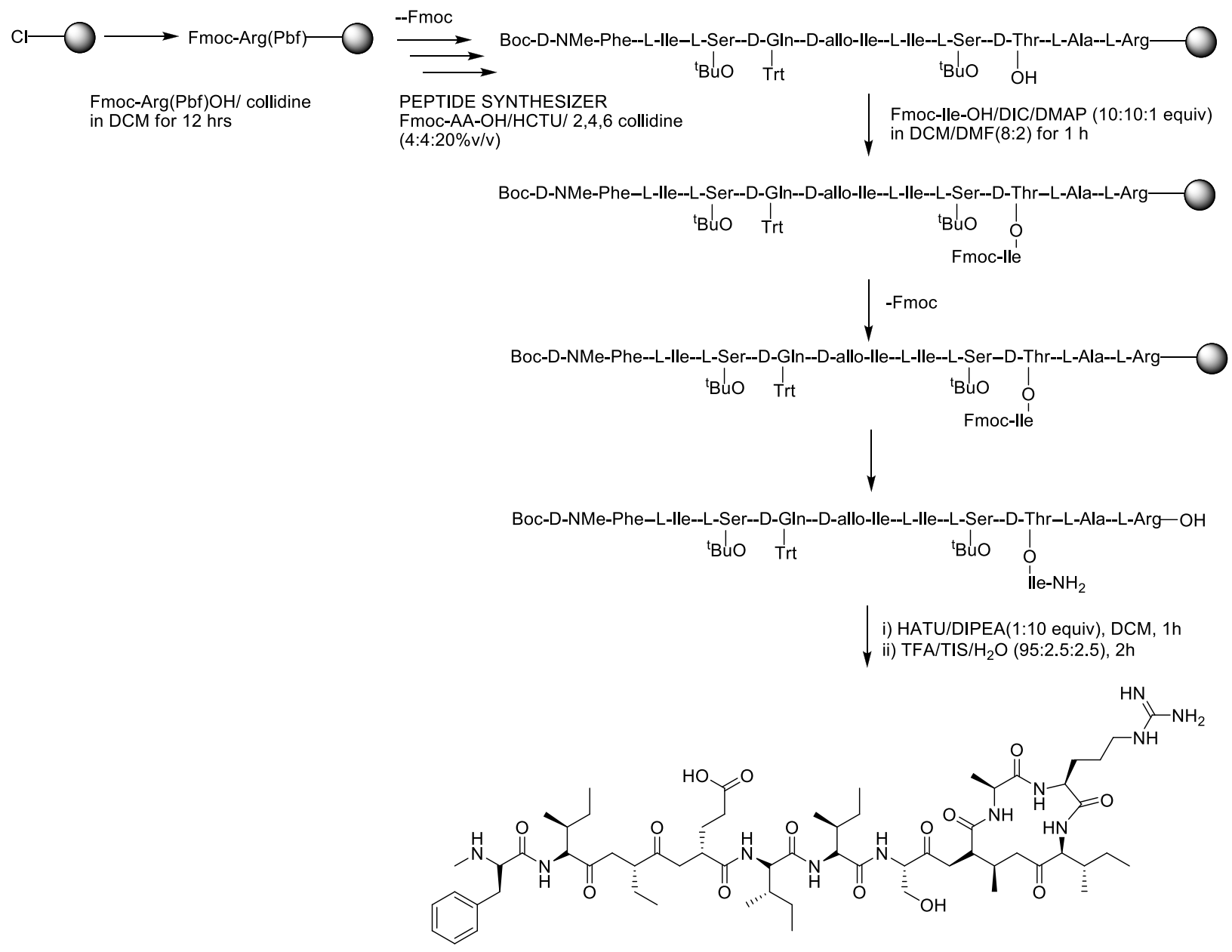

Figure 4.3 Scheme used by Yang et al, to synthesize different teixobactin analogs. ${ }^{104}$ 
Another synthesis was published by Yang et al. ${ }^{105}$ on teixobactin analogs. They used same resin, i.e., 2-chlorotriyl chloride resin, as the previous two groups. Rather than attaching alanine at C-terminus, they started synthesis with $\mathrm{L}$-arginine as the first amino acid. The following amino acids were alanine and D-threonine without side chain hydroxyl group protection. The remaining residues on the linear chain were coupled as per solid phase peptide synthesis. Then lactonization between unprotected alcohol group of D-threonine and carboxyl group of Fmoc-Ile-OH were conducted. Their method has demonstrated that it is possible to conduct O-acylation of D-Threonine on the full-length peptide attached to the resin beads. Previous two groups attempted this approach but were unsuccessful. This method made the esterification step easy and demonstrated that teixobactin analog could be prepared without special protecting groups, e.g., Alloc-protected amino acids. Comparable to previous approaches, they conducted cleavage under mildly acidic conditions whereby the peptide kept its side chain protecting groups, followed by solution phase macrolactamization to form an amide bond between Ile and Arg. They used a peptide synthesizer as the valuable mean to synthesize linear chain efficiently and rapidly and used it to make different analogs. Their antimicrobial studies on various teixobactin analogs revealed interesting information. Their results imply that stereochemistry of the tetrapeptide ring at the C-terminal part is important. Simultaneously, the teixobactin analog without guanidinium group can also be potently active as they demonstrated that with the lysine-substituted analog. Their studies imply if hydrophobic residues at the end of the $\mathrm{N}$-terminal chain is replaced with lipophilic dodecanoyl group the analog activity is a few times lower. ${ }^{105}$

Payne and coworkers ${ }^{97}$ synthesized teixobactin, using the approach similar to that of Jad et $\mathrm{al}^{43}$. and Palmer et al. ${ }^{104}$, as alloc protected amino acids were used. In an initial attempt, D-threonine (unprotected alcohol side chain) was first loaded onto 2-chlorotriyl resin as the first amino acid. Then esterification with L-isoleucine using myriad conditions was tested but remained unsuccessful in getting the suitable product. Esterification is one of the critical and challenging steps in the synthesis of such depsipeptides, and the steric hindrance of a $2^{\circ}$ alcohol made it particularly difficult. Then they switched from 2-chlorotrityl chloride resin to 
Nova PEG resin; it was assumed that steric bulkiness of the 2-chloro-trityl linker near the hydroxyl side group of threonine hinders esterification.

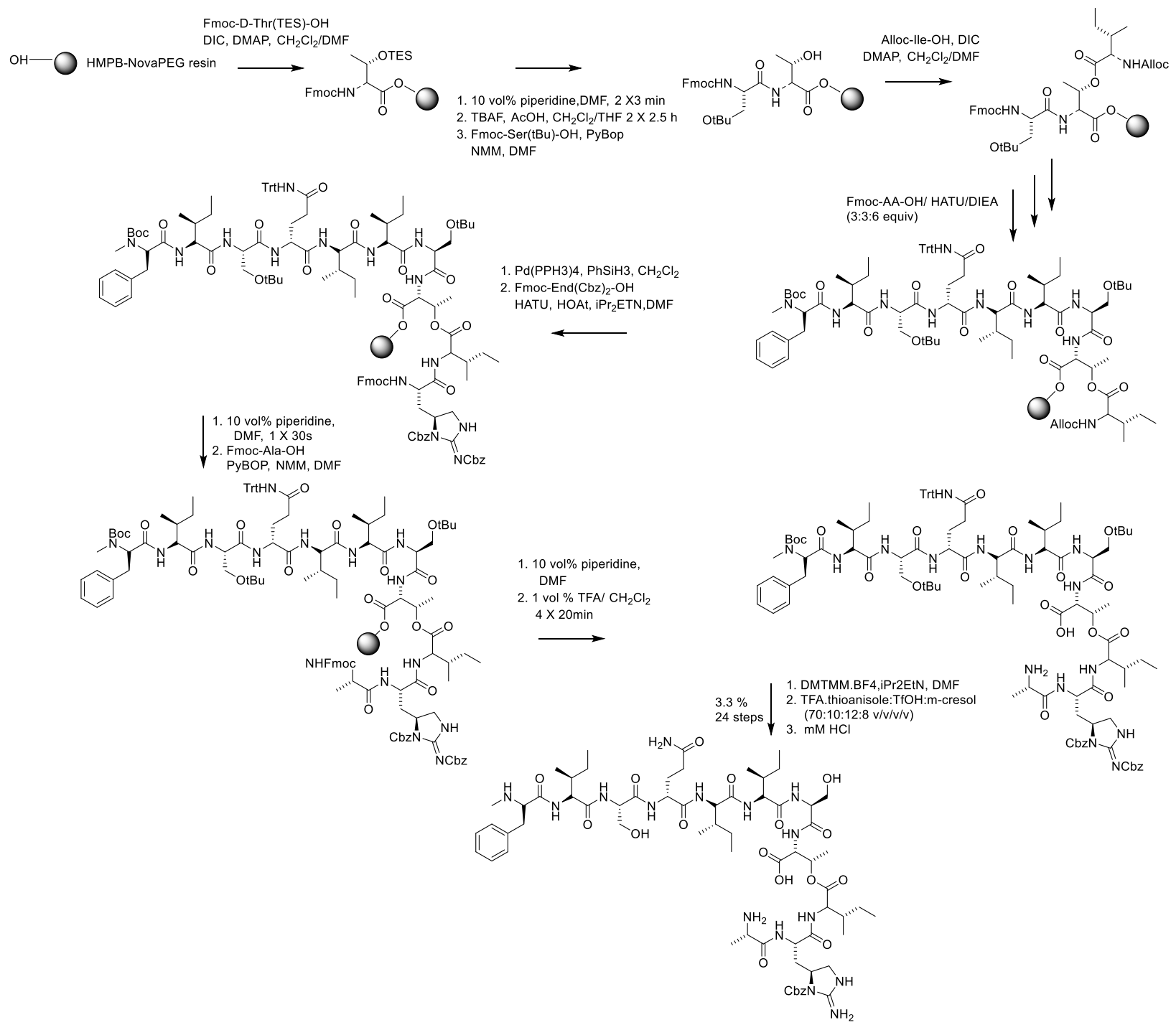

Figure 4.4 Synthesis of teixobactin analog using strategy employed by Giltrap et al. ${ }^{97}$

Jad et al. ${ }^{43}$ reported encountering similar issues during esterification. If the side-chain hydroxyl group at D-threonine is not exposed properly, it may hinder esterification. With the different approach of using Nova PEG resin, Fmoc-D-Thr(TES)-OH was coupled as the first amino acid. Fmoc protecting group was removed from $\alpha$-amine of D-Thr and trimethylsilyl(TES) was removed from the side chain to expose the hydroxy group. Fmoc- 
Ser(tBut)-OH was added as the second residue followed by the crucial step of esterification with Alloc-protected-Ile-OH. After esterification, Fmoc group on serine is deprotected and subsequent amino acids coupled to complete the methylated-N-terminal peptide chain. Then Alloc protection on esterified isoleucine is removed, and Fmoc-End(CBZ) $2-\mathrm{OH}$ is coupled. In the report, they also mentioned synthesis of L-allo-enduracidine. Then Fmoc deprotection of on the enduracidine residue for a short while to avoid diketopiperazine formation and coupling with Fmoc-L-Ala-OH using standard conditions. Then Fmoc group was removed from alanine. The cleavage of the protected peptide from the resin was conducted using $1 \%$ TFA. Once the crude protected depsipeptide was obtained, it was subjected to the key cyclization reaction to form teixobactin in $16 \mathrm{~h}$. Then the TFA deprotection cocktail was used to remove all protecting groups. After purification by HPLC and lyophilization, the final product was obtained with a yield of $3.3 \%$.

The two crucial steps in the synthesis of teixobactin or its analog are esterification and cyclization. For all the syntheses mentioned above, esterification was achieved on resin, but cyclization was done in solution. For cyclization, ring size and topology determines the extent of difficulty in its formation. Schimit and Langer reported the challenges related to tetra- and pentapeptide ring cyclization. ${ }^{106}$ In their studies, cyclization attempts often produce side products of cyclodimerization, trimerization, and $\mathrm{C}$-terminal epimerization. The ring closure reaction or macrolactamization is also sequence-dependent. A few amino acids pose major challenges for cyclization. If the site of macrolactamization has beta-branched amino acids, i.e., isoleucine or valine, the sterically encumbering residue will decrease the yield of cyclization. The macrolactamization efficiency can be improved if the reactive termini have opposite stereochemical configuration. However, in teixobactin, beta-branched isoleucine is part of the tetracyclic ring. A suitable cyclization strategy is expected to increase the synthesis yield and make this class of cyclodepsipeptides more accessible. Our methodology to address this issue is discussed in the following section. 


\subsection{Results and Discussion}

Teixobactin represents a novel target for total synthesis. It has a small cyclodepsitetrapeptide at the C-terminal part. The smaller ring size combined with a despsi bond and with a linear N-terminal tail is often challenging to synthesize. Numerous attempts have been made to identify the right scheme for the synthesis of teixobactin. Other research groups have reported efficient esterification on the resin to form the despsi bond and cyclization in solution to get the desired cyclized peptide through peptide bond formation. The ring closure process was carried out in which the linear precursor peptide had its side chain protecting groups, and that was followed by a final deprotection step to obtain the desired free peptide. ${ }^{107,86}$ Such an approach of macrolactamization has limitations. It involves multiple purification steps and often generates substantial side products and significant epimerization even with mild activation of the carbonyl group. ${ }^{106}$ Moreover, for a large peptide, the solubility of the peptides with side-chain protecting groups is a serious issue, as the protected peptide becomes extremely hydrophobic which would require a large amount of solvents for solubilization. Therefore it is not practical to use the solution cyclization strategy to synthesize analogs of cyclopeptides or preparing related compound libraries for in-depth screening. ${ }^{108}$ These limitations prompted the development of cyclization methods for minimum protected peptides but at the cost of more undesired side reaction. ${ }^{109}$ With the reported schemes for teixobactin synthesis, it would be quite challenging to introduce any further modifications post cyclization.

Solid phase cyclization would be preferable. One of the main advantages of solid phase macrolactamization is that simple washing and filtration is sufficient to remove excess reagents and byproducts while the desired cyclization product is still attached to the resin. The pseudodilution phenomenon also decreases the extent of side reactions, such as cyclodimerization. Recently, a myriad of reports are published about peptide macrolactamization on a solid support, as these methods are becoming increasingly used in the synthesis of cyclic peptides. 


\subsubsection{Strategy of our synthesis}

The aim of our work was to develop a straightforward and efficient synthetic route to prepare teixobactin and its analogs. A more attractive approach would be to form the cyclic structure on a solid phase, as has been with other regular cyclic peptides. ${ }^{110}$ So, toward this aim, we designed an on-resin cyclization strategy in which the cyclodepsitetrapeptide ring is formed while the peptide is anchored to the resin through the thiol of cysteine (Figure 4.5). After the full-length peptide sequence had been assembled, the desired teixobactin analog with a Cys in place of Ala was released from the resin after a cleavage reaction. The Cys residue also provides a site for further modification, such as dimerization through disulfide formation and thiol-alkylation. A desulfurization step would also convert the Cys residue to Ala in the native form. In this scheme, all the steps, except the desulfurization reaction, were performed on the solid phase. It is a much simpler procedure than the previously developed methods, making it much easier to introduce various modifications on the tetrapeptide ring and the $\mathrm{N}$ terminal tail. As such, our scheme provides an efficient way to synthesize teixobactin analogs and thus understand their structure-activity relationship.

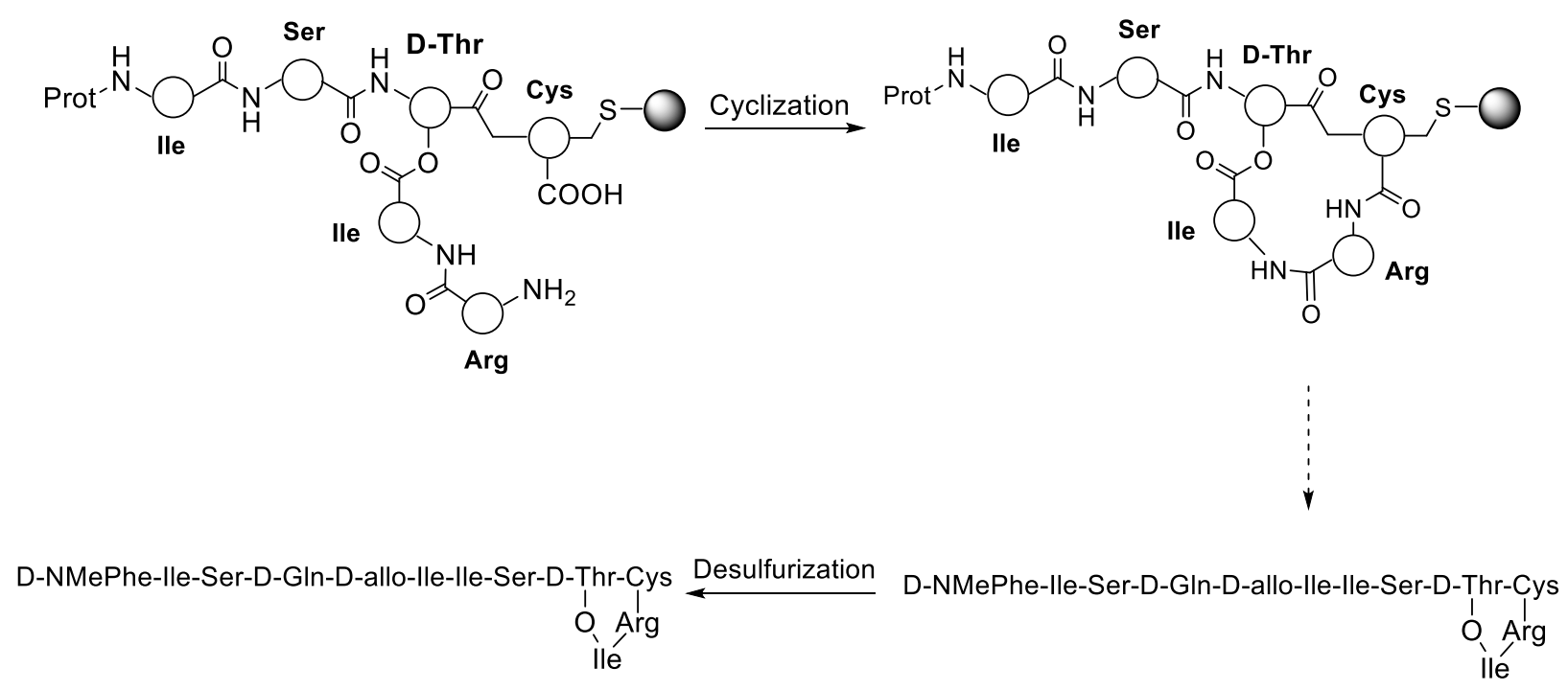

Figure 4.5 Our general strategy for the synthesis of teixobactin and its analogs.

The choice of Cys as the anchoring amino acid for resin attachment and cyclization junction is the key to the success of our strategy. It is also a distinct feature that makes this 
method different from the previously developed ones. Thiol is an excellent nucleophile, so it is very easy to load a cysteine onto a resin support through its thiol group by reacting it with a chloro-trityl linker. The advantage of using Cys is also manifested by its many interesting reactivities: desulfurization to convert Cys to Ala, oxidation to form S-S bond for dimerization and S-alkylation, all of which would be useful for the synthesis of teixobactin and analogs.

\subsubsection{Loading Fmoc-Cys-OH onto trityl resin}

To achieve this objective, commercially available Fmoc-Cys(Trt)-OH was first treated with TFA (trifluoroacetic acid) to remove the trityl protecting group. After removing TFA, the desired Fmoc-Cys-OH product was obtained with $>95 \%$ yield in powder form after lyophilization. 2-Chlorotrityl chloride resin was then treated with TFA to generate carbocation ion. The trityl carbocation resin was then treated with Fmoc-Cys-OH. The reaction mixture was kept on belly dancer overnight to yield the trityl resin loaded with Fmoc-Cys-OH, 2.

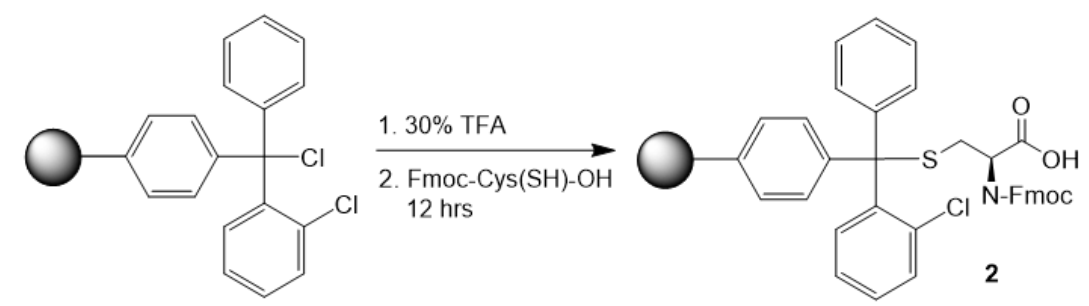

a) Formation of cysteine linked resin through the functional group.

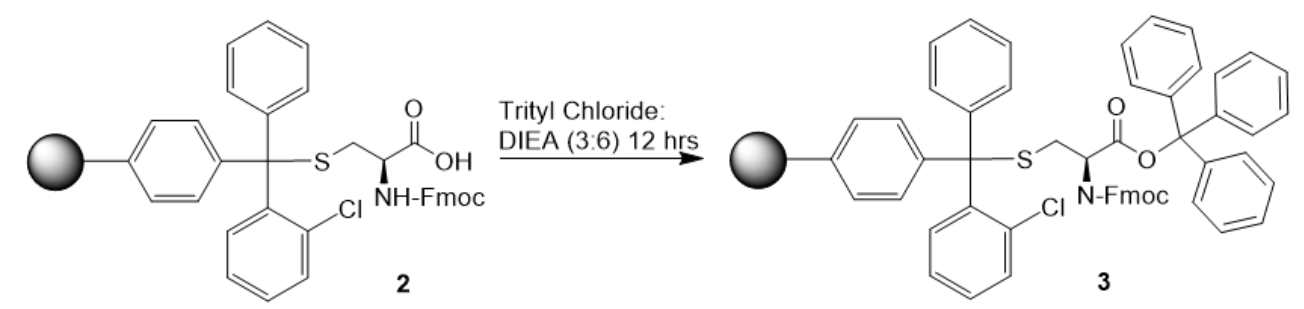

b) Trityl protection of carbonyl on cysteine linked resin

Figure 4.6 Cysteine linked resin and trityl protection a) and b). 
The carboxyl group of the cysteine was protected with trityl (or 2-chlorotrityl) group to give 3. The trityl group being very bulky can prevent the formation of diketopiperazine upon the deprotection of Fmoc on the next residue. ${ }^{111}$

\subsubsection{Azide protected isoleucine}

The synthesis of teixobactin and its analogs requires an intricate protecting group strategy. As it is obvious from Figure 4.6, the protecting group on the main peptide chain amine must be different from that on the amine of the branching peptide chain on the Thr side chain oxygen, since from this focal-point threonine peptide chain grows along its $\alpha$-amine while another branches out from its $\beta$-hydroxyl. Azide is a precursor to the primary amine via a mild reduction reaction. It is stable and can withstand reaction conditions of peptide synthesis. So it is orthogonal to Fmoc or Boc. We used azido as the masked amino group, which is orthogonal to the Fmoc group used on the branching peptide chain on Thr. Azidoisoleucine was synthesized with a one-step reaction in more than $95 \%$ yield, as shown below.

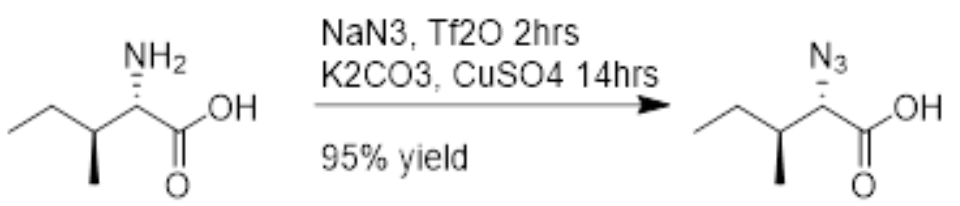

Figure 4.7 Preparation of azido-isoleucine.

Azide is light-sensitive. Once it was used in peptide synthesis, the reaction vessel was wrapped in aluminum foil. It can be converted to the amine with ease. We found that exposing azido-protected peptide on solid phase beads to TCEP solution easily could readily reduce the azide to an amine.

\subsubsection{Optimization of the on-rein esterification reaction}

Esterification on the $\beta$-hydroxyl of Thr by isoleucine was a quite challenging and very crucial step of the synthesis. Different peptidyl-resins and several methods were tested; in our experience, esterification of the Thr $\beta-\mathrm{OH}$ in a shorter peptide was more feasible than in a 
longer peptide. Esterification on the tripeptide or tetrapeptide substrate on solid phase was achieved after testing various conditions. Both Fmoc-Ile-OH and $\mathrm{N}_{3}$-Ile-OH were tested for the O-acylation of Thr.
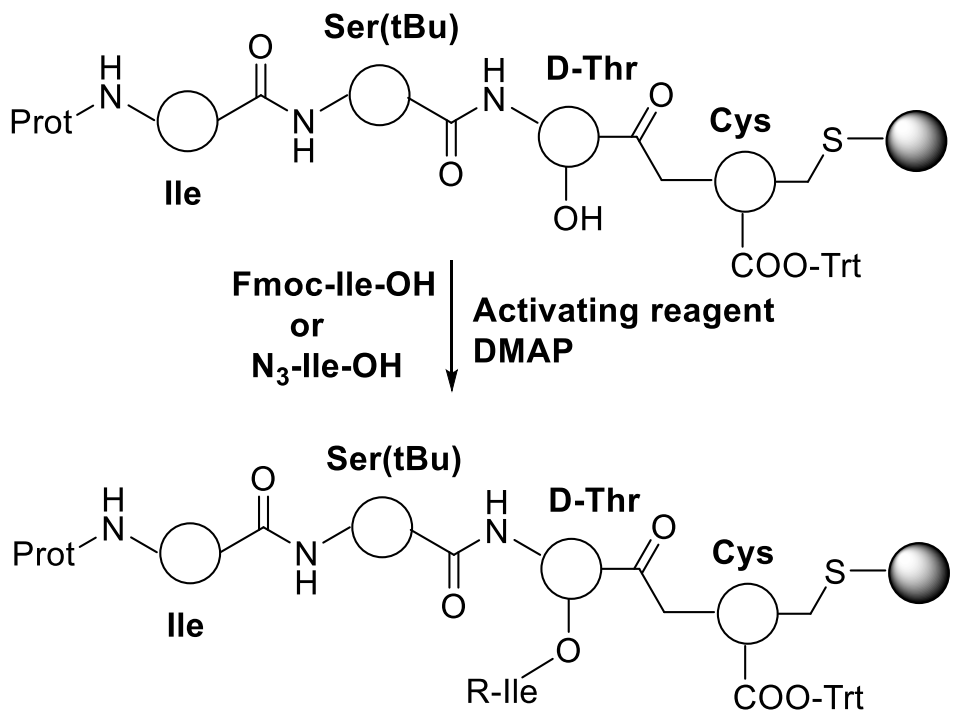

Figure 4.8 A representative esterification reaction scheme on solid phase.

For teixobactin synthesis, esterification depends on many factors including resin, linker, the position of threonine and the length of the peptide chain. We prepared a number of peptide substrates to test the esterification conditions (Table 4.1). Peptide-resin 1 was prepared with nine amino acid residues to proceed with esterification. Unfortunately, results were not satisfactory after multiple attempts. When exposed to the strong activating agent, chloro$\mathrm{N}, \mathrm{N}, \mathrm{N}$ ', N'-tetramethylformamidiniumhexafluoro-phosphate, a small amount $(\sim 25 \%)$ of esterification product was obtained after analyzing the cleavage mixture from the resin. Esterification of the same peptide-resin $\mathbf{1}$ with other activating reagents also gave poor results (data not shown). Peptide-resin 2 which has the complete 11 amino acid residues gave very poor results as well. These results suggest esterification of the $\mathrm{Thr} \beta-\mathrm{OH}$ in a relatively long peptide on the solid phase is not favorable. 
Table 4.1 Optimization of on-resin esterification conditions with different peptides.

\begin{tabular}{|c|c|c|c|}
\hline No & Peptide on resin & Esterification conditions & yield \\
\hline 1 & $\begin{array}{l}\text { Boc- dF-I-S-dQ-dI-I-S-dT(OH)- } \\
\text { C(2Cl-trityl-resin)-Trt }\end{array}$ & $\begin{array}{l}\text { Fmoc-Ile-OH/ chloro-n n n' n'- } \\
\text { tetramethylformamidiniumhexafluorophosphate/DMAP(4:4:0.4) } \\
18 \mathrm{~h}\end{array}$ & $\sim 25 \%$ \\
\hline 2 & $\begin{array}{l}\text { Boc-S-dT(OH)-C(2Cl-trityl-resin)- } \\
\text { Trt }\end{array}$ & $\mathrm{N}_{3}$-Ile-OH/DIC/DMAP (5:5:0.5) $18 \mathrm{~h}+$ DIC 5equiv $37^{\circ} \mathrm{C}$ & $\sim 90 \%$ \\
\hline 3 & $\begin{array}{l}\mathrm{N}_{3}-\mathbf{I}-\mathbf{S}-\mathbf{d T}(\mathrm{OH})-\mathbf{C}(2 \mathrm{Cl} \text {-trityl resin)- } \\
\text { Trt }\end{array}$ & Fmoc-Ile-OH/DIC/DMAP (10:10:1), 2 h, r.t & $\sim 90 \%$ \\
\hline 4 & $\begin{array}{l}\mathrm{N}_{3}-\mathbf{I}-\mathbf{S}-\mathbf{d T}(\mathrm{OH})-\mathbf{C}(2 \mathrm{Cl} \text {-trityl resin)- } \\
\text { Trt }\end{array}$ & Fmoc-Ile-OH/DIC/DMAP (10:5:1), 2 h, r.t & $\sim 90 \%$ \\
\hline 5 & $\begin{array}{l}\mathrm{N}_{3} \text {-I-S-dT(OH)-C(2Cl-trityl resin)- } \\
2 \text { Cl-Trt }\end{array}$ & Fmoc-Ile-OH/DIC/DMAP (8:8:0.8), 2 h, r.t. & $\sim 90 \%$ \\
\hline 6 & $\begin{array}{l}\text { Fmoc-S-dT(OH)-C(2Cl-trityl resin) } \\
-2 \mathrm{Cl} \text {-Trt }\end{array}$ & $\mathrm{N}_{3}-\mathrm{Ile}-\mathrm{OH} / \mathrm{DIC} / \mathrm{DMAP} \quad(5: 5: 0.5), 6 \mathrm{~h}$, r.t. & $\sim 90 \%$ \\
\hline
\end{tabular}

However, when the esterification was done on a much shorter resin-bound peptide, such as 3-7, excellent yields of the ester products were obtained. It seemed that the hydroxyl group of the Thr in the short resin-bound peptides was much more accessible than in the long resin-bound peptides. The use of an excess amount of the carboxyl acid (i.e., Fmoc-Ile-OH or $\mathrm{N}_{3}$-Ile-OH) and DIC as the activating agent in the presence of DMAP gave optimal results (Table 4.1). For instance, HPLC analysis of the crude cleavage mixture after esterification of 5 revealed a good purity profile of the ester product (Figure 4.9).
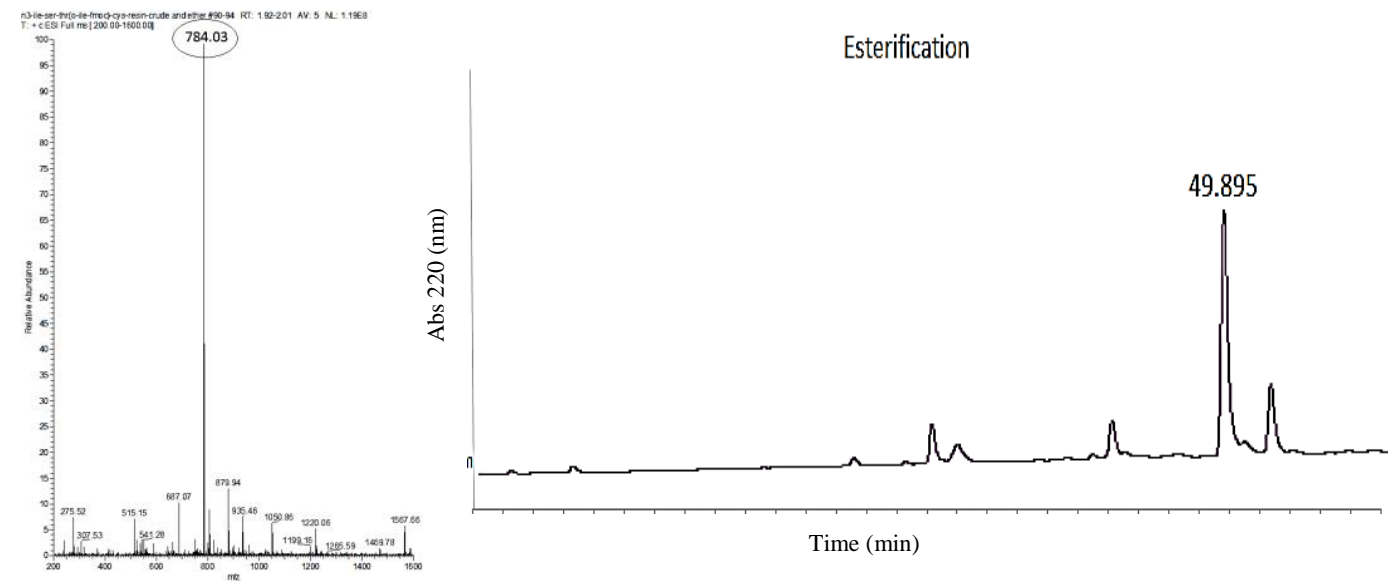

Figure 4.9 Analytical HPLC profile (right panel) and ESI-MS of the crude ester product of peptide 5. Observed $\left[\mathrm{M}+\mathrm{H}^{+}\right]^{+} \mathrm{m} / \mathrm{z}=784.03$. 


\subsubsection{Two routes for the synthesis of teixobactin analogs}

After optimizing esterification conditions, the synthesis could go on by two different routes depending on when to form the cyclic depsitetrapeptide ring. Figure 4.10 illustrates the two routes using the Arg10 analog as an example.

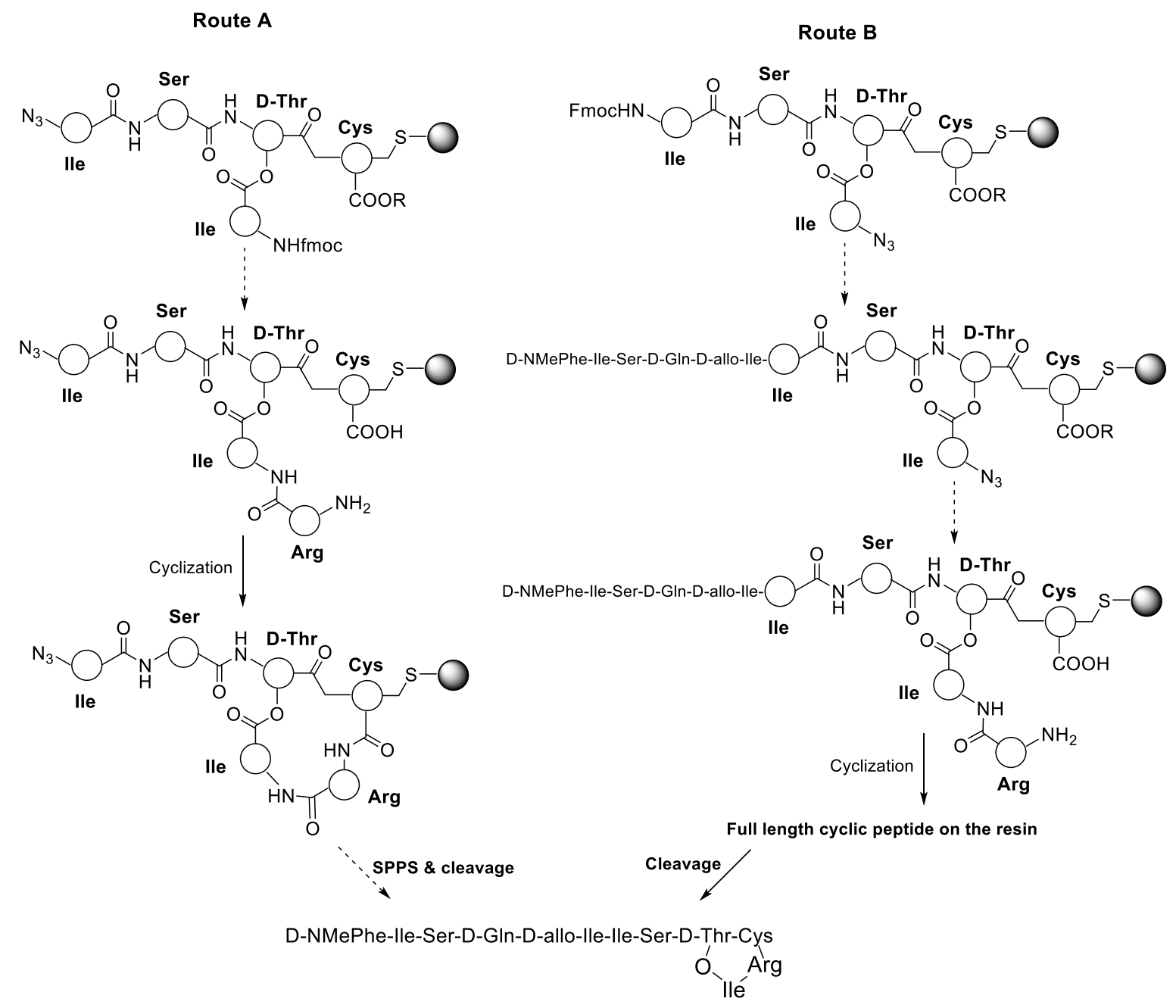

Figure 4.10 Two routes to synthesize teixobactin using the on-resin cyclization approach. $\mathrm{R}=$ trityl or 2-Cltrityl.

One route is to form the cyclodepsipeptide ring first, then proceed with SPPS on the main chain to assemble the full sequence to give the final product after cleavage (Route A, Figure 4.10); the other is to assemble the full sequence first, then form the cyclodepsipeptide 
ring structure to yield the desired product after the cleavage reaction (Route B, Figure 4.10). We employed both routes and compared their advantages.

\subsubsection{Synthesis of the Arg10-teixobactin analogs by synthetic route A}

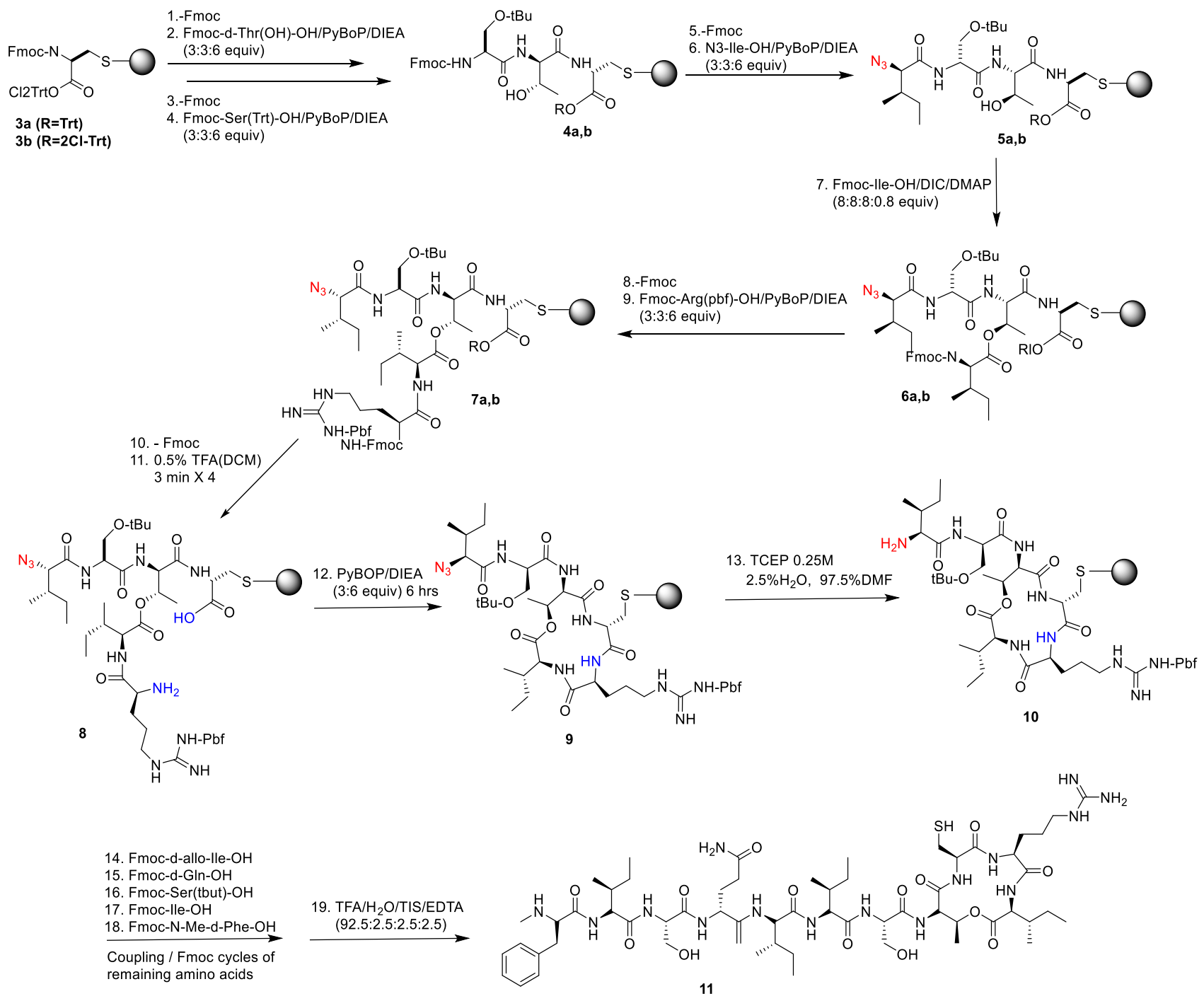

Figure 4.11 Synthesis of the Arg10-analog of teixobactin via Route A. 


\subsubsection{The choice of protecting group for the C-ter $\mathrm{COOH}$}

From section 4.2.2 (Figure 4.6), once $\mathbf{3}$ was obtained, three cycles of conventional solid phase peptide synthesis were carried out to afford 5. Esterification was then performed with Fmoc-Ile-OH, DIC and DMAP (8:8:08 equiv) to give the ester product 6. When analyzing the cleaved product of $\mathbf{6 a}$, we observed some side products which were most likely related to the instability of the trityl protecting group of the Cys $\mathrm{COOH}$. One of the side product was a $+67 \mathrm{Da}$ adduct which presumably was formed from the coupling of piperidine to the prematurely deprotected $\mathrm{COOH}$ of the $\mathrm{C}$-terminal Cys residue. Because the trityl ester of a $\mathrm{COOH}$ is extremely acid-sensitive, exposure to HOBt and/or Fmoc-amino acid would knock it off during the coupling/esterification reactions. For this reason, we changed trityl to 2-Cl-trityl for the protection of the $\mathrm{Cys} \mathrm{COOH}$ because the 2-Cl-trityl ester of $\mathrm{COOH}$ is more stable. The use of the 2-Cl-trityl protecting group in $\mathbf{3 b}$-7 $\mathbf{7 b}$ essentially solved the instability problem of the trityl ester.

\subsubsection{Macrolactamization}

Teixobactin has a depsitetrapeptide ring at its C-terminal part. The formation of a cyclotertapeptide like this is known to be quite difficult because of the high angle strain of such small rings. Side reactions like cyclodimerization are often observed when the cyclization reaction is done in solution. Therefore, high dilutions are required to avoid such side reactions for solution cyclization, which however would complicate the work-up procedures after the reaction. It is advantageous to conduct cyclization on solid phase due to the pseudo-dilution effects, which has been used to prepare many cyclic peptides. ${ }^{112}$ In our case, the 2-Cl-trityl ester in $\mathbf{7 b}$ was deprotected with $0.5 \%$ TFA for 2-3 minutes and repeated 3 times, followed by a quick $0.75 \%$ TFA exposure for a few seconds. The thioether linkage of the trityl resin seems to be stable under these conditions. The cyclization was performed on 8 (Figure 4.11) with PyBOP and DIEA for $6 \mathrm{~h}$. 
A micro-cleavage was conducted to monitor the pre and post-cyclization status of the peptide on the resin. The HPLC graphs (Figure 4.12) show the clean conversion of the linear peptide 8 to the cyclized peptide 9. The HPLC profile pre-cyclization (Fig. 4.12 top) revealed two peaks at RT $37.501 \mathrm{~min}$ and $38.715 \mathrm{~min}$ which corresponded to the desired linear products of the same MW. We attributed the minor peak at 38.715 min to the epimerization product of Ile11 formed during the esterification step.
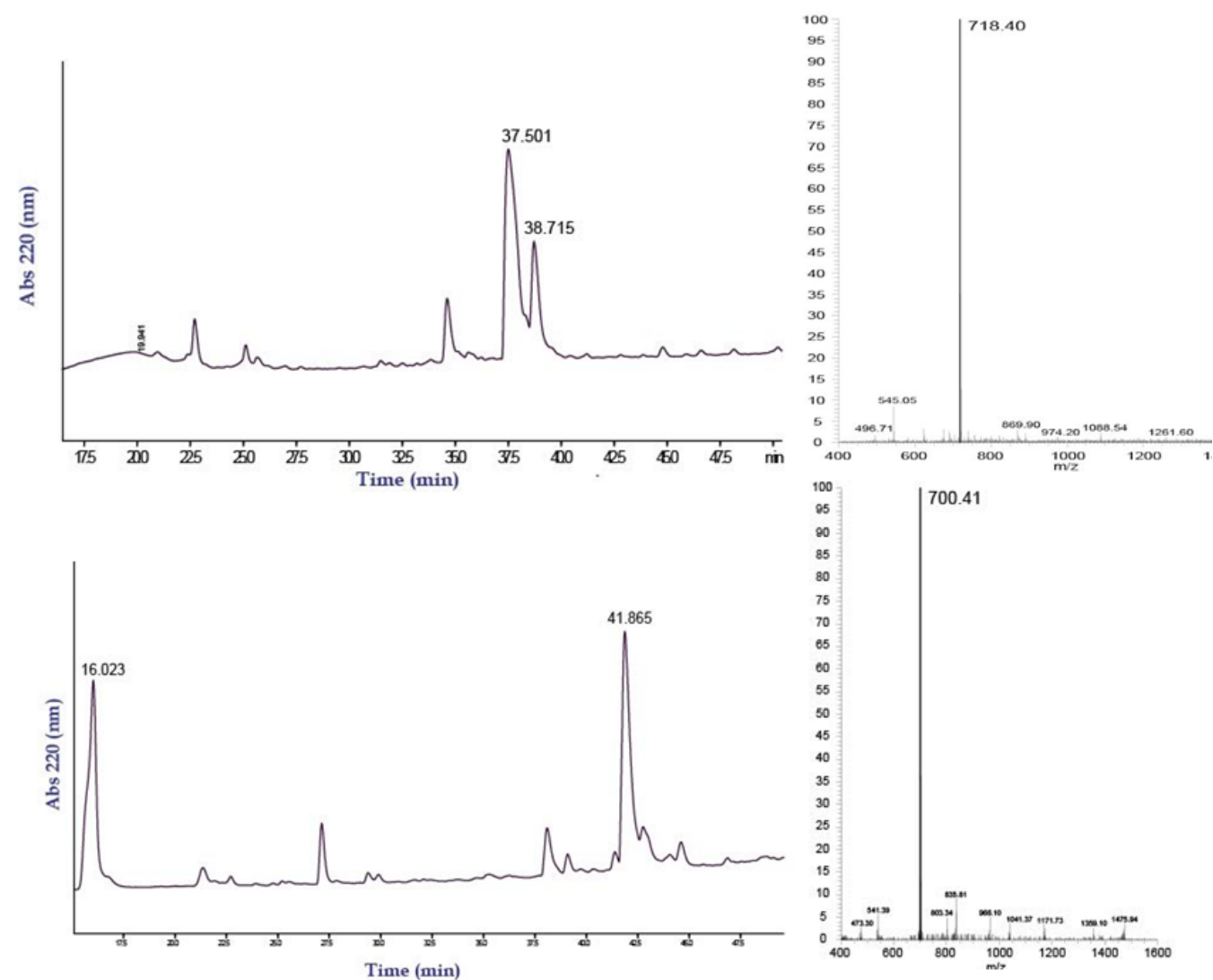

Figure 4.12 HPLC and MS monitoring of the cyclization reaction on a solid phase. Peptide 8 (top) before cyclization and the cyclized peptide 9 (bottom).

After cyclization, the azide group of $\mathbf{9}$ was reduced with TCEP in DCM to form $\mathbf{1 0 .}$ Kaiser test confirmed the presence of the free amine and microcleavage was also conducted to confirm azide to amine conversion. The remaining amino acids were coupled to assemble the full-length peptide. Fmoc was removed from the secondary amine of N-Me-D-Phe. The peptide resin was then treated with the TFA cleavage cocktail. The desired peptide $\mathbf{1 1}$ was 
approximately $20 \%$ of the crude product. Figure 4.13 shows the HPLC profile and MS spectrum of the purified teixobactin analog: Cys9, Arg10-teixobactin.
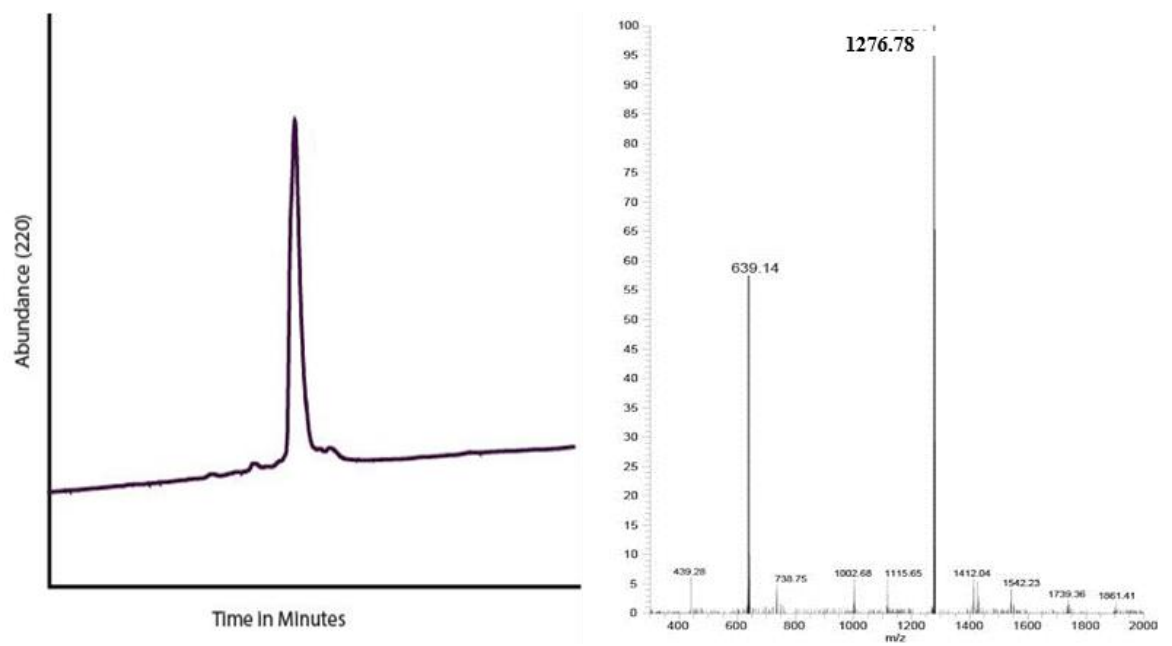

Figure 4.13 Analytical HPLC profile (left panel) and the ESI-MS (right panel) of pure Cys9, Arg10teixobactin 11. Observed $\left[\mathrm{M}+\mathrm{H}^{+}\right]^{+}=1276.78$.

\subsubsection{Desulfurization}

In this scheme, the 11-mer peptide obtained after cleavage from the resin has a cysteine rather than alanine at position 9. A single step of desulfurization reaction can convert cysteine to alanine. The Thiol functional group also gives an opportunity for further modifications. Thiol-containing peptides have the tendency to dimerize in oxidizing conditions. So dimeric analogs of teixobactin can be easily prepared from the Cys-containing teixobactin.

Different conditions were tested to determine optimum desulfurization conditions. There are several desulfurization methods. In this work, we used Raney nickel as the reagent in the desulfurization of the synthesized teixobactin peptides. Two different types of reaction conditions: one was to use $20 \% \mathrm{AcOH} / \mathrm{H}_{2} \mathrm{O}$ as the solvent and the other was to use guanidinium hydrochloride/phosphate buffer solution in the presence of TCEP (Figure 4.14). Both types of conditions gave quite an efficient desulfurization with more than $90 \%$ conversion to afford the desulfurized product, as also reported with other peptides. ${ }^{113}$ 


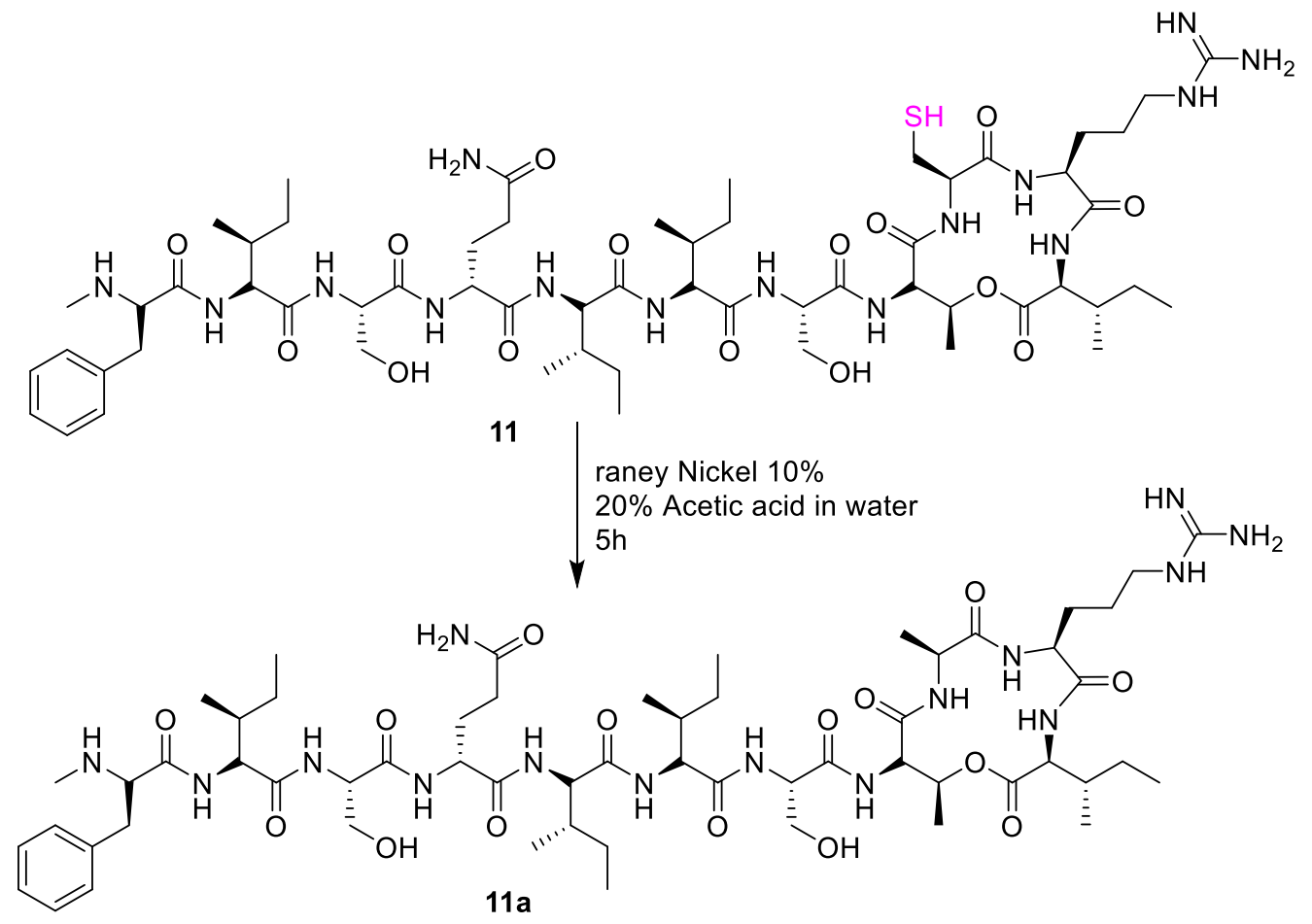

Figure 4.14 Desulfurization of Cys9,Arg10-teixobactin 11 to afford Arg10-teixobactin.
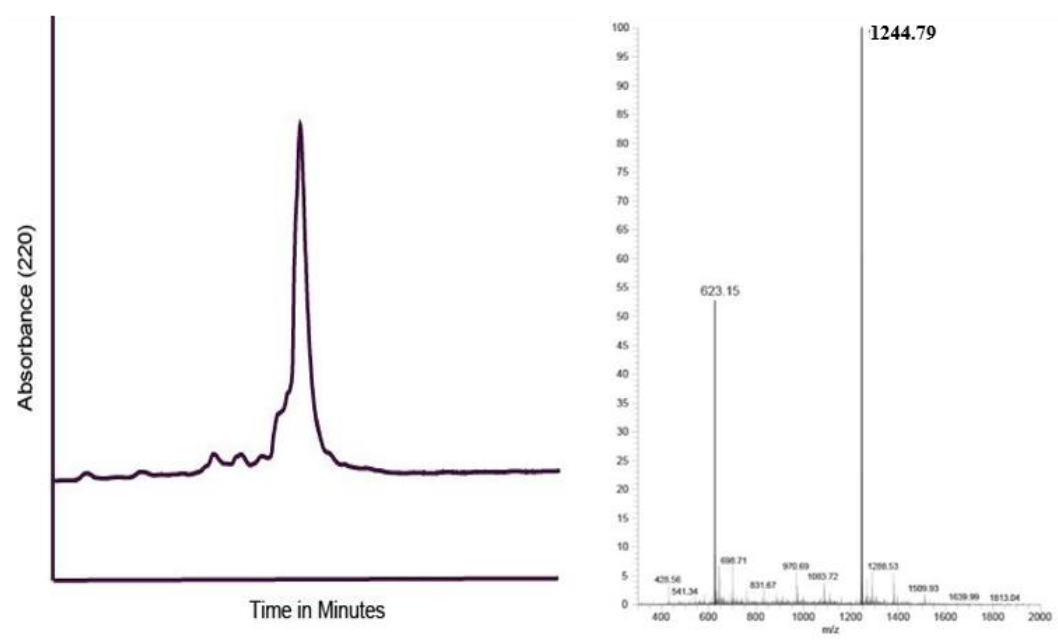

Figure 4.15 Analytical HPLC profile (left) of the desulfurization reaction mixture using $20 \% \mathrm{AcOH}$ solution and ESI-MS (right) of the isolated desulfurization product 11a. $\left[\mathrm{M}+\mathrm{H}^{+}\right]^{+}=1244.79$. 
In one condition, $20 \%$ acetic acid aqueous solution was used as a medium for desulfurization. Raney nickel was freshly prepared before starting the reaction. As Raney nickel is in the slurry form, it is hard to weigh it accurately. Therefore, approximately $10 \%$ volume of Raney nickel of the total reaction solution volume was added. $20 \%$ acetic acid helped solubilization and recovery of the compounds. The reaction took place for $5 \mathrm{~h}$. The Desulfurized product usually has a slight early shift in retention time. This general procedure was utilized for the desulfurization of other analogs as well. Figure 4.15 shows the HPLC analysis of the desulfurization reaction mixture under this condition. The ESI-MS analysis confirms the required product. For further characterization, the desulfurized product Arg10teixobactin 11a was analyzed with NMR and HRMS. The observed $\left[\mathrm{M}+\mathrm{H}^{+}\right]^{+}: 1244.7340$ and calculated $\left[\mathrm{M}+\mathrm{H}^{+}\right]^{+}:$1244.7367. The NMR and HRMS spectra are shown in the appendix of the thesis.

Desulfurization can also be conducted with guanidinium hydrochloride phosphate buffer ( $\mathrm{pH}$ 6). An approximate $10 \%$ of Raney nickel to the reaction volume was used, and TCEP was added. Without adding TCEP, the peptide could self-dimerize due to oxidation,

and the dimer was observed by HPLC analysis. Although the conversion yield was similar to the previous condition of using acetic acid solution, the recovery yield of the product was poorer.

\subsubsection{The dimer of Cys9,Arg10-teixobactin, 11b}

The cysteine residue can be easily oxidized to form the disulfide-linked dimer $\mathbf{1 1 b}$ (Figure 4.16). Since the oxidation occurred spontaneously in air, some amount of the dimer 11b was isolated during the purification of the Cys9,Arg10-teixobactin product 11. 


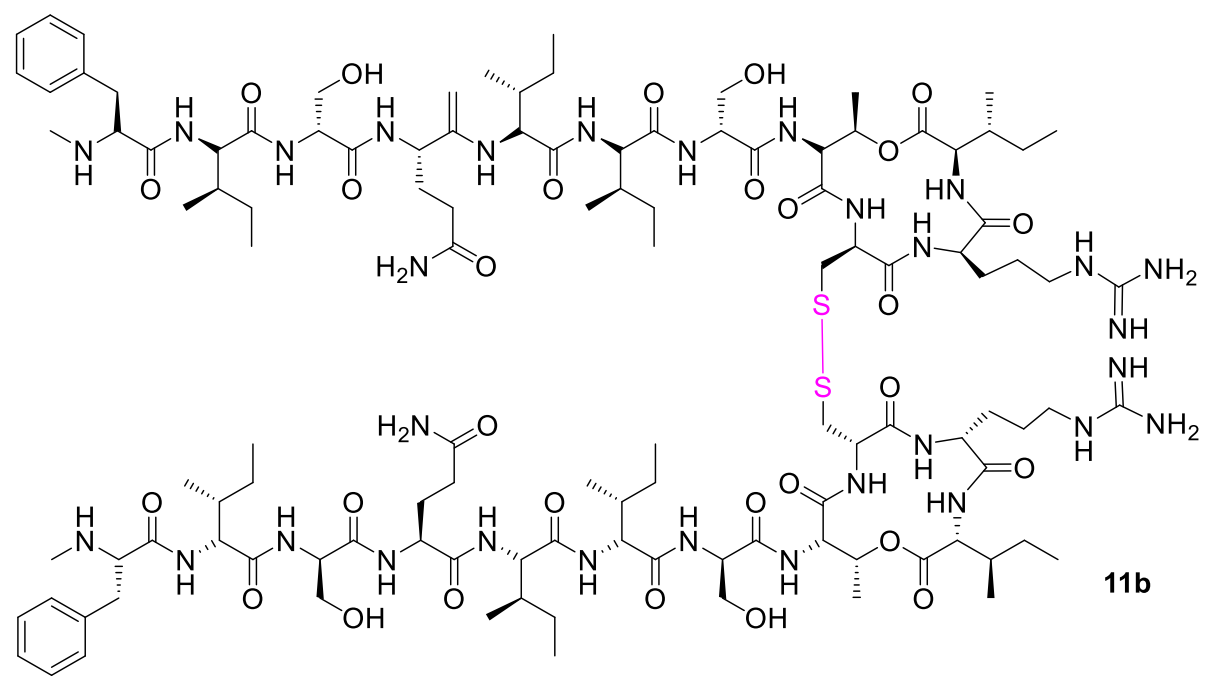

Figure 4.16 Structure of the dimer of Arg-teixobactin formed through a disulfide bond.
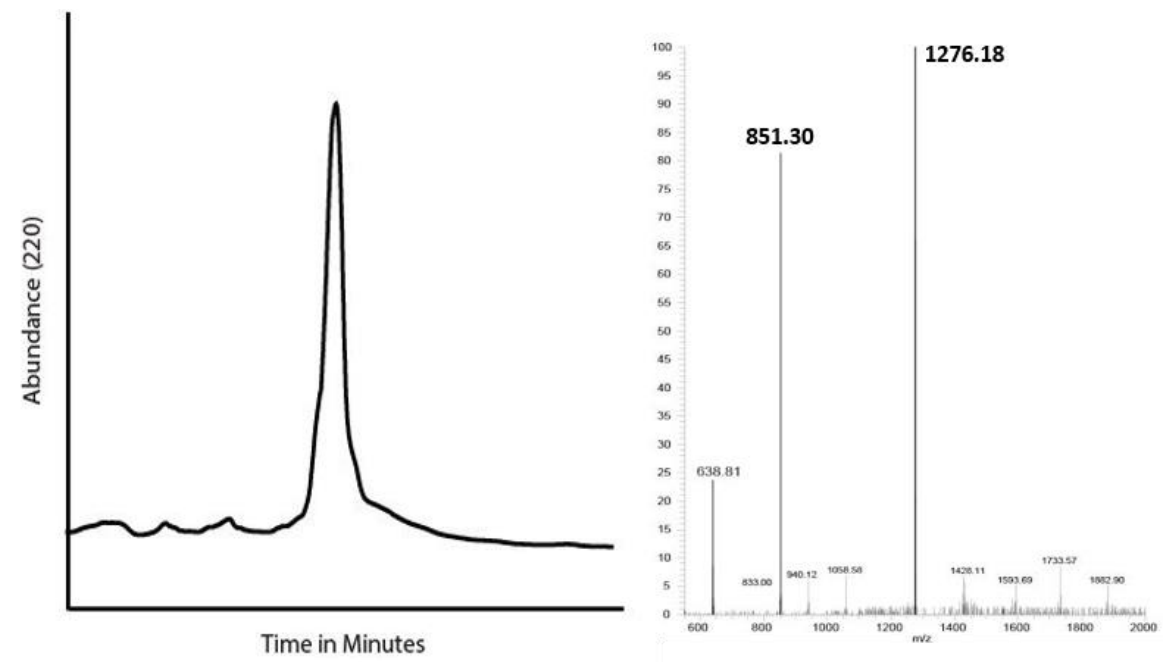

Figure 4.17 Analytical HPLC profile (left) of the S-S Arg-teixobactin dimer 11b and its ESI-MS spectrum (right). Observed $\left[\mathrm{M}+\mathrm{H}^{+}\right]^{+}=1276.76 \&\left[\mathrm{M}+\mathrm{H}^{+}\right]^{+}=851.30$

\subsubsection{The synthesis of other analogs with Lys, Orn or His at position 10}

Using the synthetic route $\mathbf{A}$, other analogs were synthesized as well. These analogs also have a basic residue at position 10, which is Lys, Orn or His. Replacing enduracidine/arginine with these residues can elucidate the importance of the side-chain guanidine in the tetrapeptide ring. 


\subsubsection{Formation of the cyclic depsipeptide ring in the synthesis of the Lys, Orn or His10 analogs of teixobactin}

So, using route A (4.11), after peptide 6 b was synthesized, Fmoc-Lys(Boc)-OH, Fmoc-Orn(Boc)-OH or Fmoc-His(Trt)-OH was coupled to the peptidyl-resin followed by the critical cyclization process. The cyclization was done using the same procedure. Figure 19 below shows the structure of the hexapeptides containing the cyclodepsitetrapeptide ring.

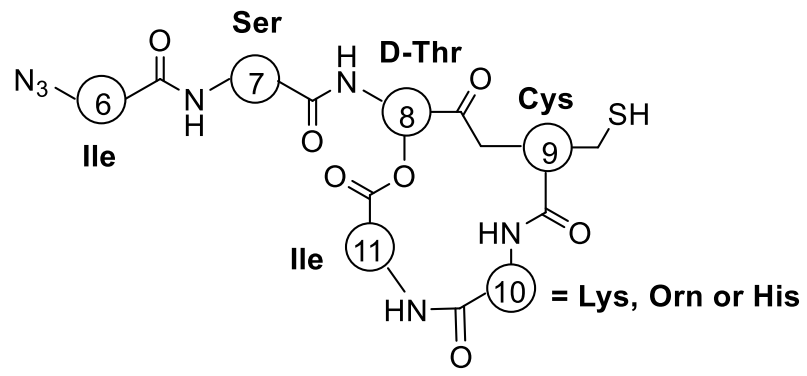

Figure 4.18 Structure of the hexapeptides with the C-ter cyclodepsitetrapeptide ring. The thick red bond was formed at the on-resin cyclization step.

One can see that the on-resin cyclization reaction proceeded smoothly from HPLC analysis of the pre and post-cyclization products of the hexapeptides after microcleavage (Figure 4.19-21).
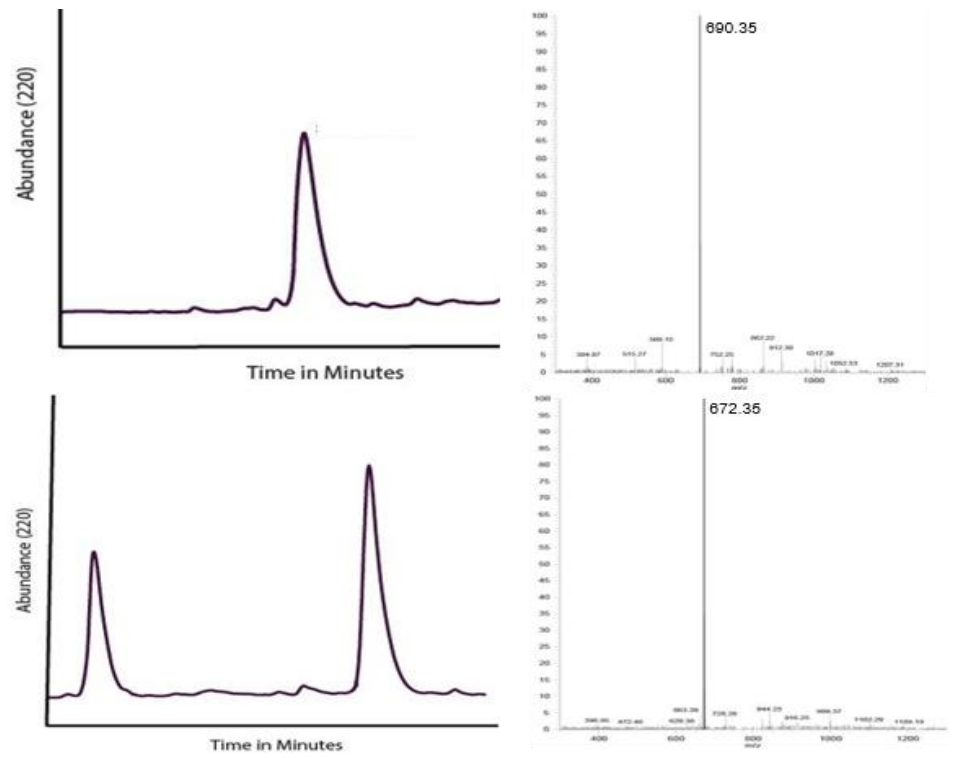

Figure 4.19 HPLC profile of the linear hexapeptide with Lys10 pre-cyclization (top) and the cyclic product post-cyclization (bottom) after microcleavage. The left panel shows analytical HPLC profile and right panel shows ESI-MS spectra. Observed $\left[\mathrm{M}+\mathrm{H}^{+}\right]^{+}=690.35$ (linear); $\left[\mathrm{M}+\mathrm{H}^{+}\right]^{+}=672.35$. 

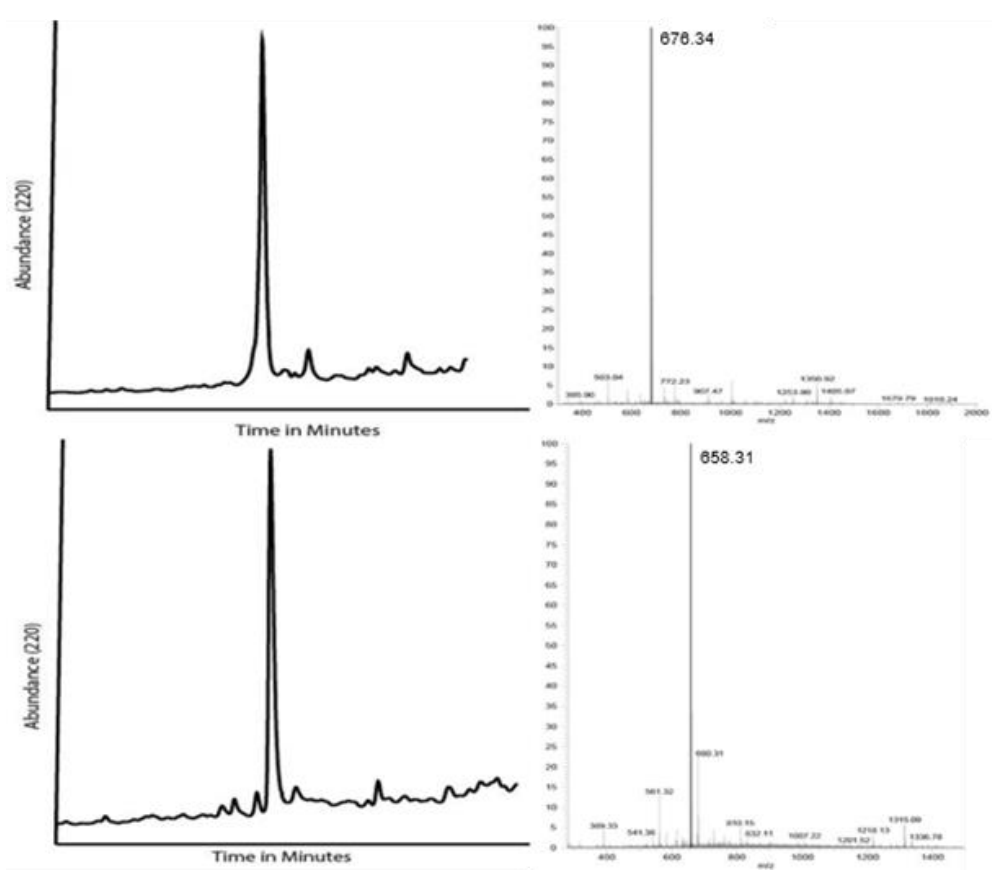

Figure 4.20 HPLC profile of the linear hexapeptide with Orn10 pre-cyclization (top) and the cyclic product post-cyclization (bottom) after microcleavage. The left panel shows analytical HPLC profile and right panel shows ESI-MS spectra.
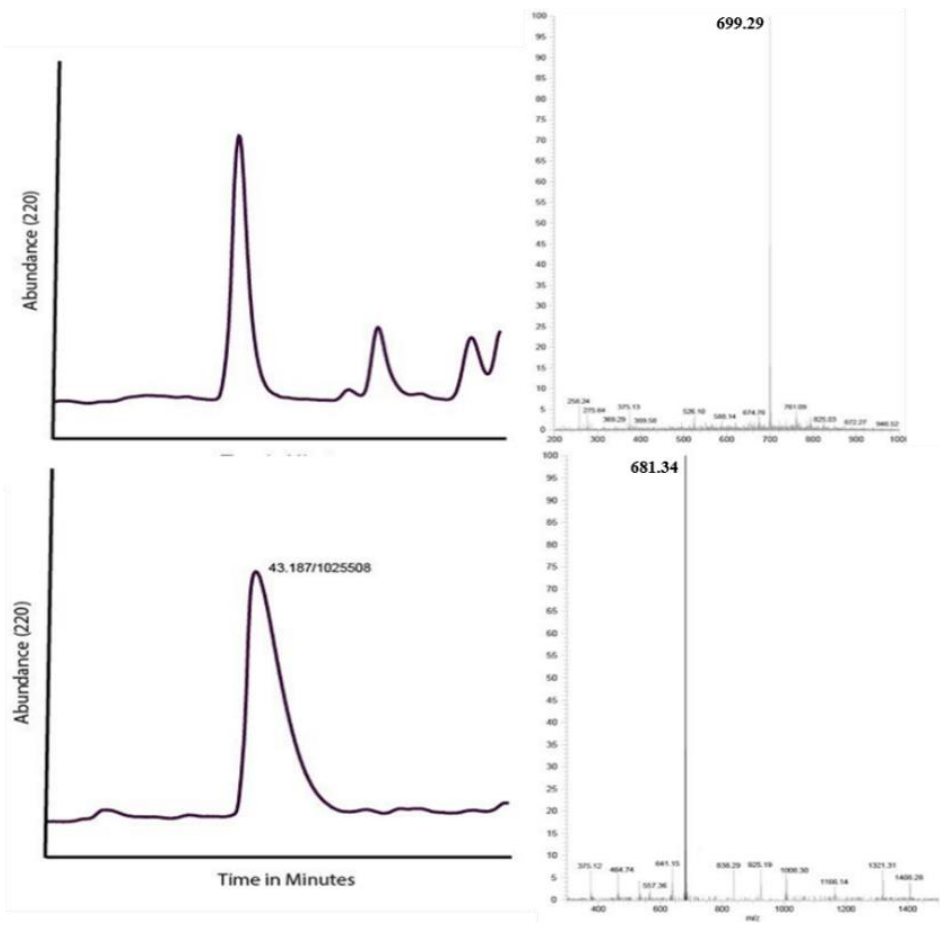

Figure 4.21 HPLC profile of the linear hexapeptide with His10 pre-cyclization (top) and the cyclic product post-cyclization (bottom) after microcleavage. The left panel shows analytical HPLC profile and right panel shows ESI-MS spectra. 
The cyclization causes slightly late elution of the peptide in HPLC. In the linear peptide before cyclization, the free amine and carboxyl contribute to hydrophilicity. After cyclization, the free amine and the carboxyl are utilized to form an amide bond, the peptide becomes more hydrophobic and thus elutes out later from the HPLC column. From the HPLC analysis; we did not see another significant peak beside the main peak with the same MW as the cyclized product, suggesting the Cys residue did not undergo significant epimerization. This was surprising as Cys is prone to racemization when activated. The likely reason for the lack of epimerization is that the formation of the highly constrained tetrapeptide ring strongly depends on the stereochemistry of the amino acids in the ring such that the cyclotetrapeptide containing the D-Cys epimer cannot easily form due to unfavorable energetics.

\subsubsection{Completing the synthesis of Cys9,Lys10-teixobactin, Cys9,Orn10- teixobactin, and Cys9,His10-teixobactin}

After the on-resin cyclization step, the azide group on isoleucine was reduced to the amine. Moreover, the remaining amino acids were coupled till completion of the full-length peptide. The peptide was deprotected and cleaved from the resin using the TFA cleavage cocktail solution. The crude peptide was purified by semi-preparative HPLC and the final product was confirmed by analytical HPLC and mass spectrometry.

The desired Lys10-teixobactin peptide 12 represented almost $16 \%$ of the crude product. The Cys9,Lys10-teixobactin structure is shown in Figure 4.22 and its analytical HPLC and ESI-MS are shown in figure 4.23.

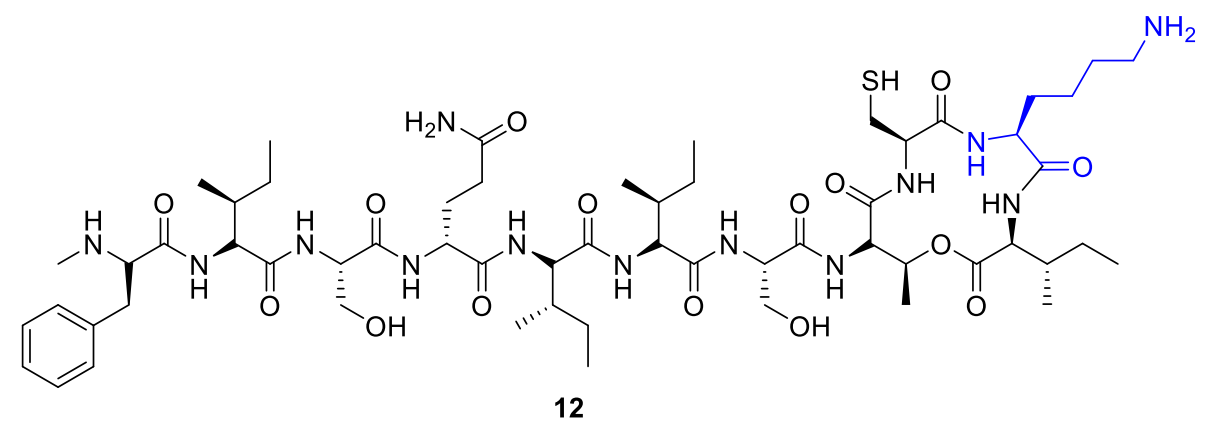

Figure 4.22 Lys10 analog of teixobactin 12 . 

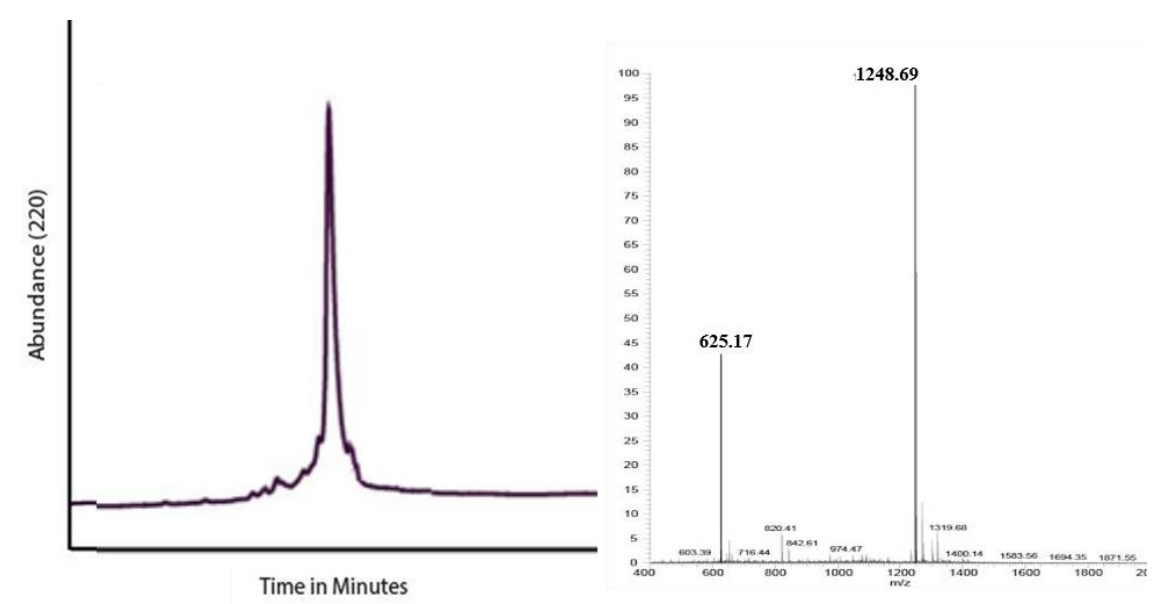

Figure 4.23 Analytical HPLC profile (left) and ESI-MS mass spectrum (right) of Cys9,Lys10-teixobactin. Observed $\left[\mathrm{M}+\mathrm{H}^{+}\right]^{+}=1248.69$.

Ornithine is structurally close to lysine, except it has one carbon less in its side chain than lysine. This analog can elucidate the importance of varying carbon chain length at the critical position 10 which has L-allo-enduracidine in the natural compound. Therefore, synthesis of ornithine teixobactin analog will help us to further understand the importance of the side chain of the amino acid at position 10 of teixobactin.

The cleaved crude product had almost $15 \%$ of the desired Cys9,Orn10-teixobactin (Figure 4.24). It's analytical HPLC and ESI-MS mass spectrum is shown in Figure 4.25.

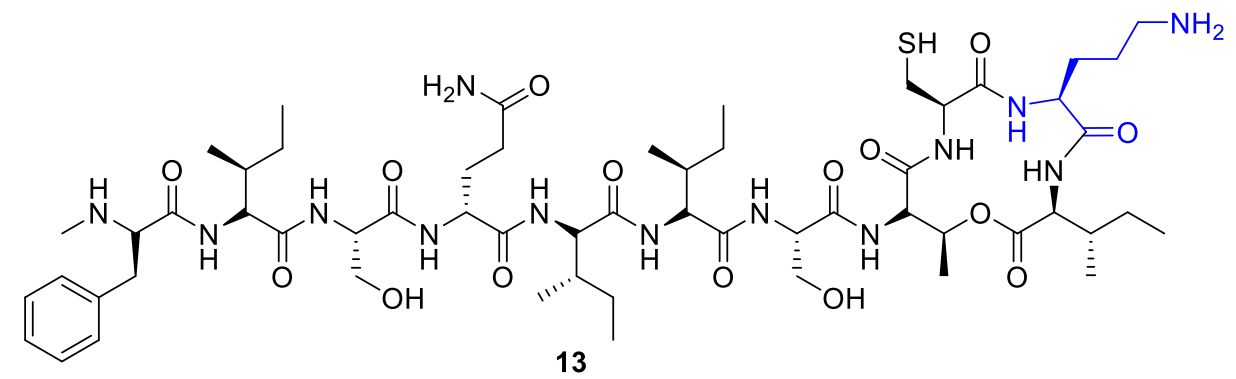

Figure 4.24 Structure of Cys9, Orn10-Teixobactin 13. 

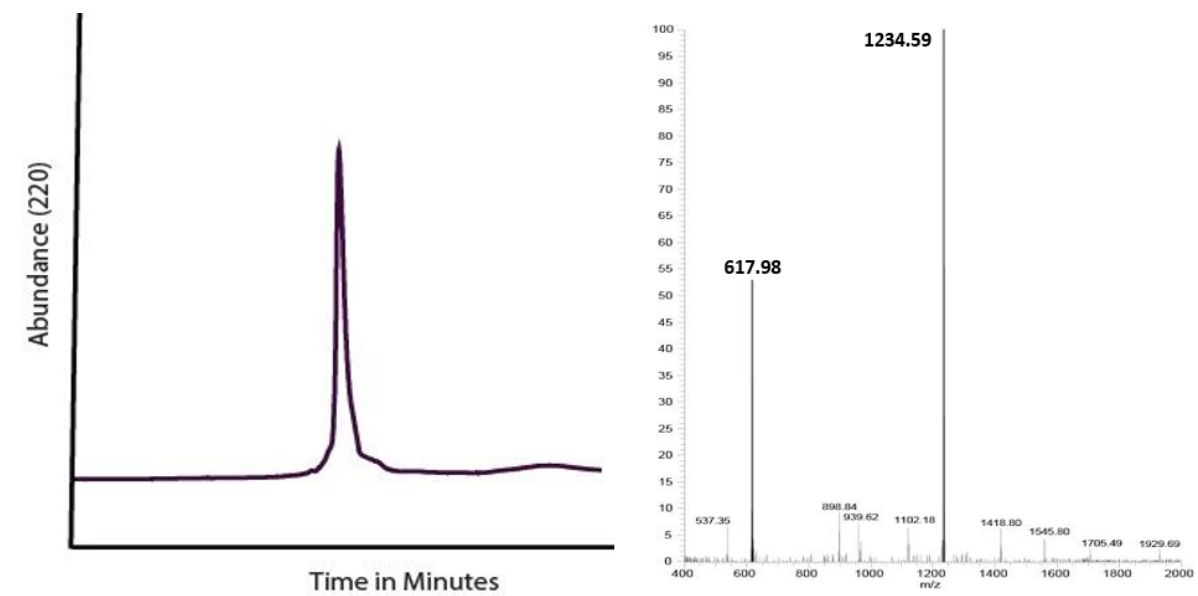

Figure 4.25 Analytical HPLC (left) of Cys9, Orn10-teixobactin 13 and its ESI-MS mass spectrum (right). Observed $\left[\mathrm{M}+\mathrm{H}^{+}\right]^{+}=1234.59$.

Histidine has a weakly basic imidazole con its side chain. We wanted to see whether having His at position 10 would affect the activity of teixobactin. The Cys9, His10Teixobactin was synthesized in a similar way as for previous analogs. $8 \%$ of the desired product was purified from the crude peptide after cleavage. Its structure is shown in Figure 4.26 and its analytical HPLC profile and ESI-MS are shown in Figure 4.27.

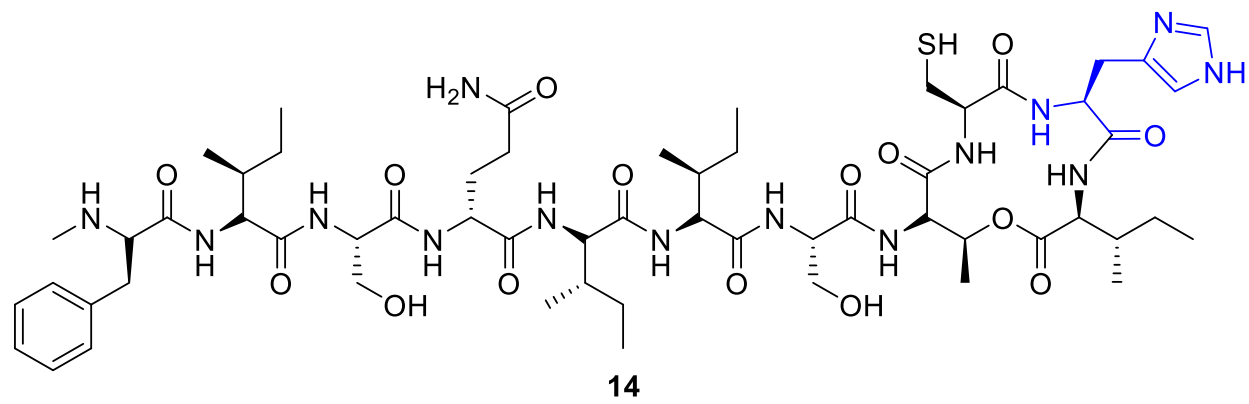

Figure 4.26 Structure of Cys9, His10-Teixobactin 14. 

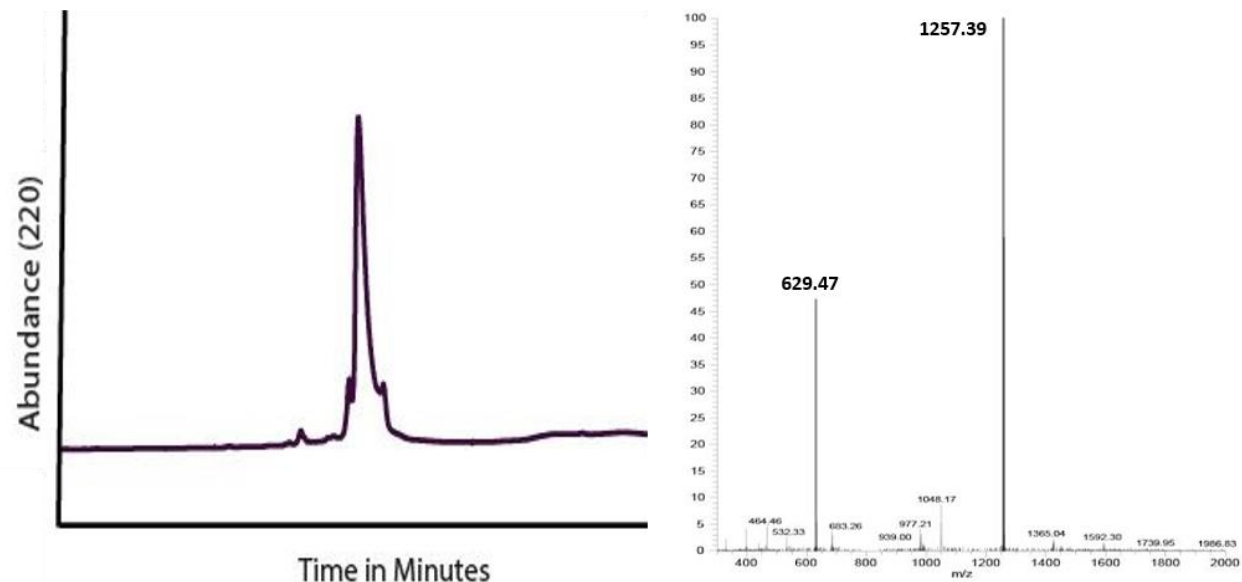

Figure 4.27 Analytical HPLC (left) and ESI-MS mass spectrum (right) of Cys9,His10-teixobactin. Observed $\left[\mathrm{M}+\mathrm{H}^{+}\right]^{+}=1257.39$.

\subsubsection{Desulfurization of Cys9,Lys10-teixobactin (12), Cys9,Orn10- teixobactin (13), and Cys9,His10-teixobactin (14)}

The Analogs synthesized were subjected to desulfurization reaction. Desulfurization was conducted using similar conditions as mentioned in the section 4.2.6.3. The desulfurized peptide eluted slightly earlier than its precursor. The peptides 12, 13, 14 were desulfurized to yield Lys10-teixobactin 12a, Orn10-teixobactin 13a, and His10-teixobactin 14a, respectively. Figure 4.28 shows the analytical HPLC profile and ESI-MS spectrum of Lys10-teixobactin 12a.
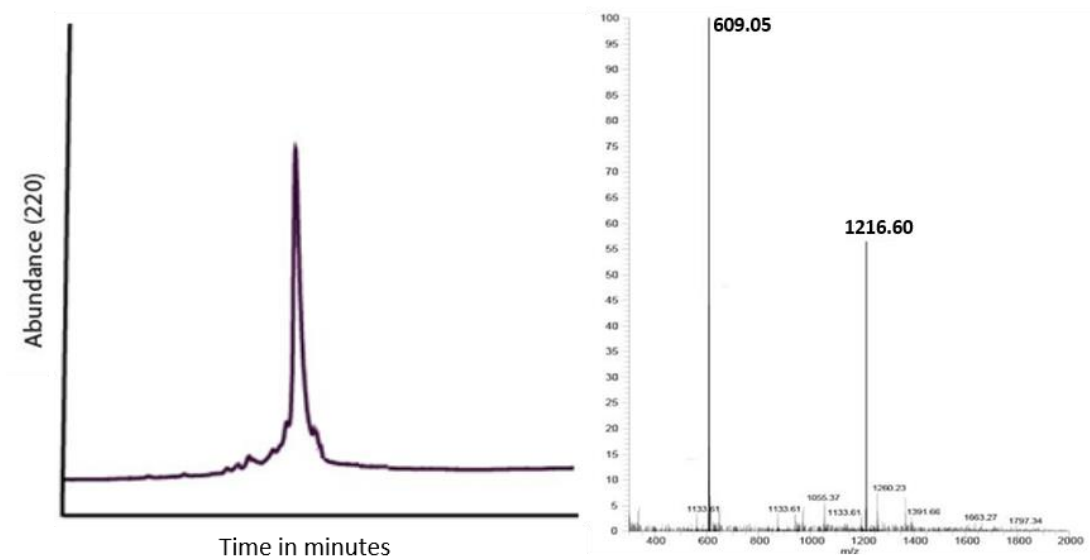

Figure 4.28 Analytical HPLC (left) and ESI-MS spectrum of Lys10-teixobactin 12a. Observed $\left[\mathrm{M}+\mathrm{H}^{+}\right]^{+}=$ 1216.60 . 
The desulfurized product 12a was further characterized using NMR and HRMS. The observed $\left[\mathrm{M}+\mathrm{H}^{+}\right]^{+}: 1216.7286$ and calculated $\left[\mathrm{M}+\mathrm{H}^{+}\right]^{+}:$1216.7305. The NMR and HRMS spectra are shown in the appendix of the thesis.

Cys9,Orn10-teixobactin 13 was desulfurized to give Orn10-teixobactin 13a. The analytical HPLC and ESI-MS of the Orn10-teixobactin (13a) is shown in Figure 4.29.
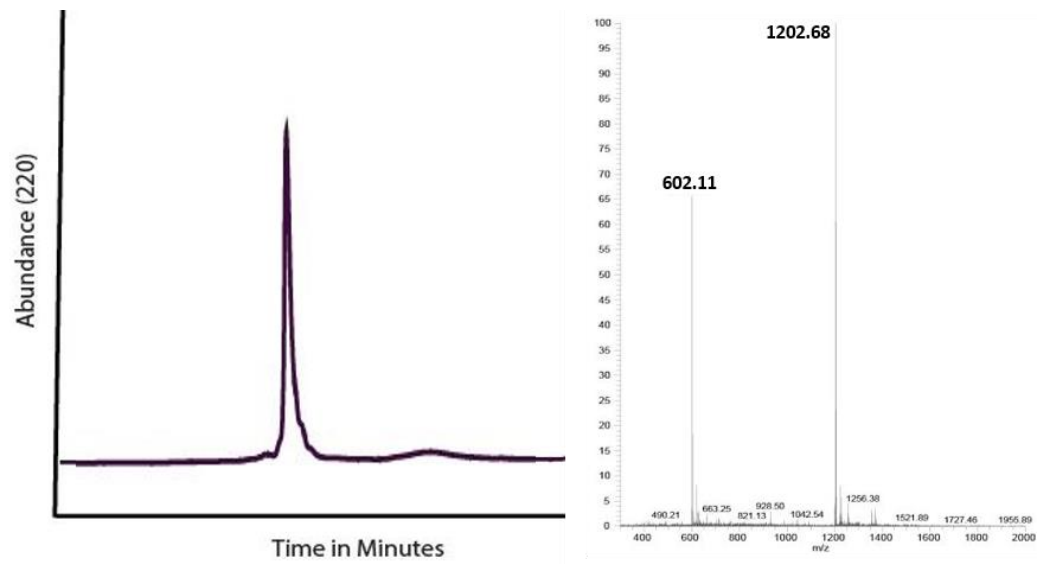

Figure 4.29 Analytical HPLC profile (left) of Orn10-teixobactin 13a and its ESI-MS mass spectrum (right). The observed $\left[\mathrm{M}+\mathrm{H}^{+}\right]^{+}=1202.68$.

The desulfurized product 13a was further characterized using NMR and HRMS. The observed $\left[\mathrm{M}+\mathrm{H}^{+}\right]^{+}: 1202.7137$ and calculated $\left[\mathrm{M}+\mathrm{H}^{+}\right]^{+}:$1202.7149. The NMR and HRMS spectra are shown in the appendix of the thesis.

His10-teixobactin (14a) was obtained after desulfurization of 14. Figure 4.30 shows the analytical HPLC profile and ESI-MS of the final product. The synthesis of the His 10 analogs gave more side products at most reaction steps. The same was true for the desulfurization step. Nevertheless, 14a was purified from the reaction mixture. It's analytical HPLC and ESI-MS spectrum are shown in Figure 4.30. The desulfurized product 14a was further characterized using NMR and HRMS. The observed $\left[\mathrm{M}+\mathrm{H}^{+}\right]^{+}: 1225.6943$ and calculated $\left[\mathrm{M}+\mathrm{H}^{+}\right]^{+}: 1225.6945$. The NMR and HRMS spectra are shown in the appendix of the thesis. 

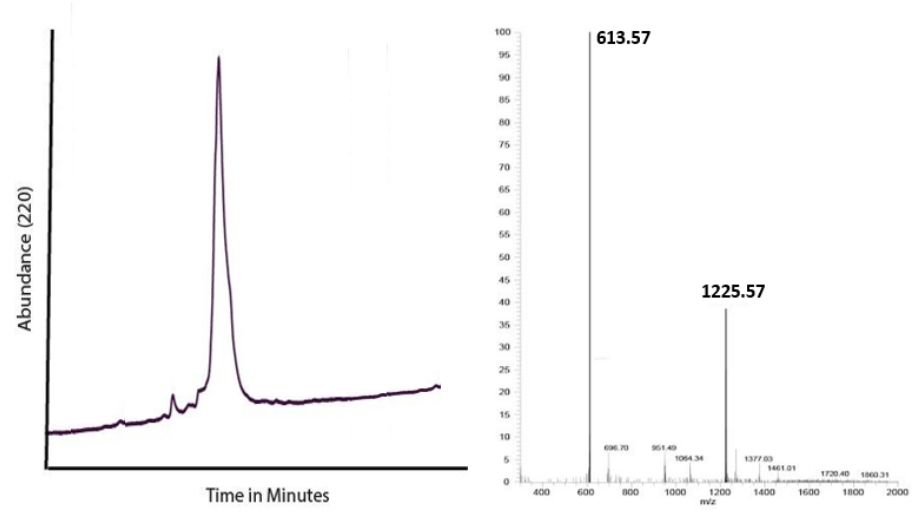

Figure 4.30 Analytical HPLC (left) and ESI-MS (right) of His10-teixobactin 14a. Observed $\left[\mathrm{M}+\mathrm{H}^{+}\right]^{+}=$ 1225.57.

\subsubsection{The dimers of Cys9,Lys10-teixobactin, Cys9,Orn10-teixobactin, and Cys9,His10-teixobactin}

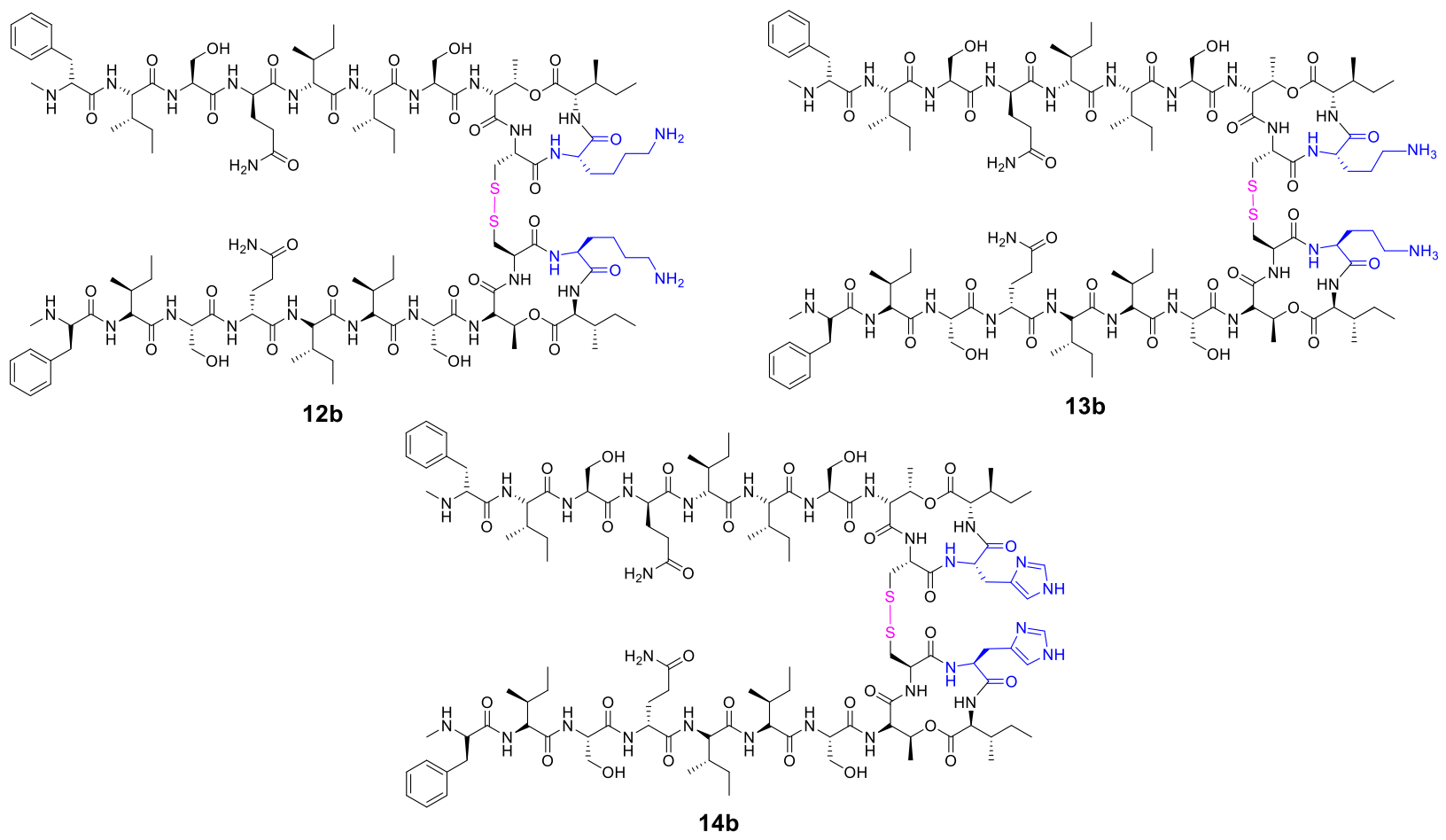

Figure 4.31 Structures of S-S dimers 12b, 13b and 14b. 
The cysteine residue at position 10 in the analogs 12, 13 and 14 can undergo oxidation spontaneously in air. As a result, some amount of the dimer 12b, 13b and $\mathbf{1 4 b}$ were isolated during the purification of the Cys10 analogs 12, 13 and 14. The structures of the three dimers are shown in Figure 4.31.

The analytical HPLC and MALDI-TOF MS of Cys9, Lys10-teixobactin dimer 12b is shown in the Figure 4.32. The dimer isolated during purification is obtained due to oxidation of 12 occurred in the air.
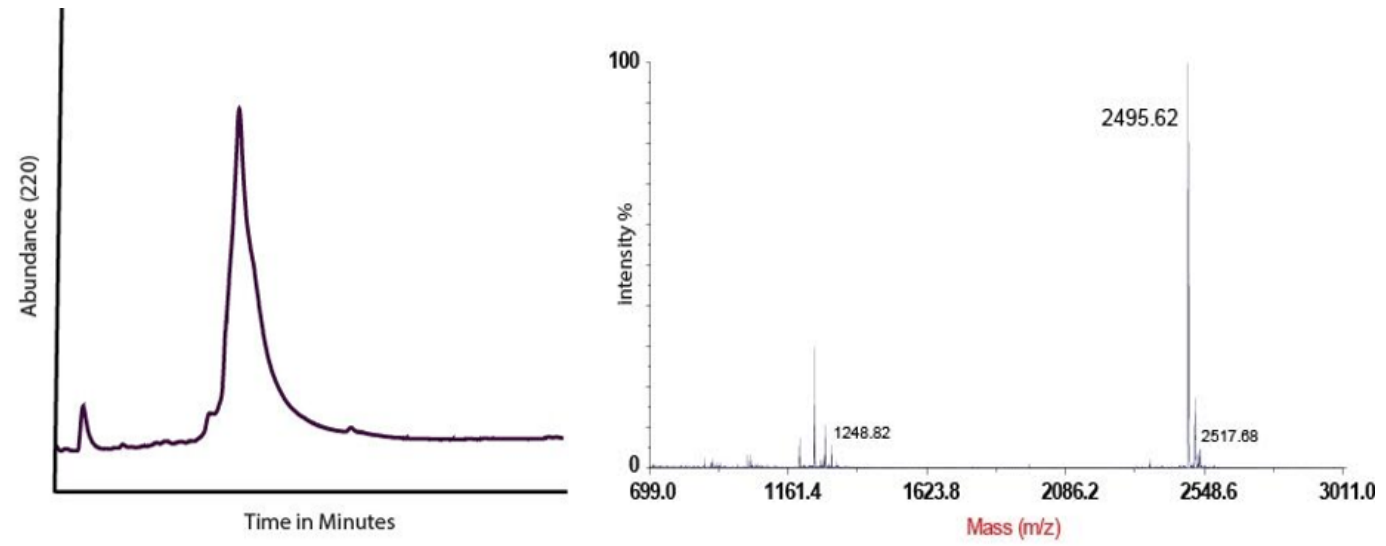

Figure 4.32 Analytical HPLC (left) of $\mathbf{1 2 b}$ its MALDI-TOF MS (right). Observed $\left[\mathrm{M}+\mathrm{H}^{+}\right]^{+}=2495.62$.

Similar to 12b, 13b and 14b were isolated and analyzed by HPLC and MALD-TOF MS. The Cys9,Orn10-Teixobactin dimer 13b and Cys9,lys10-teixobactin dimer 14b analytical HPLC and MALDI-TOF MS are shown in Figure 4.33 and 4.34, respectively. 

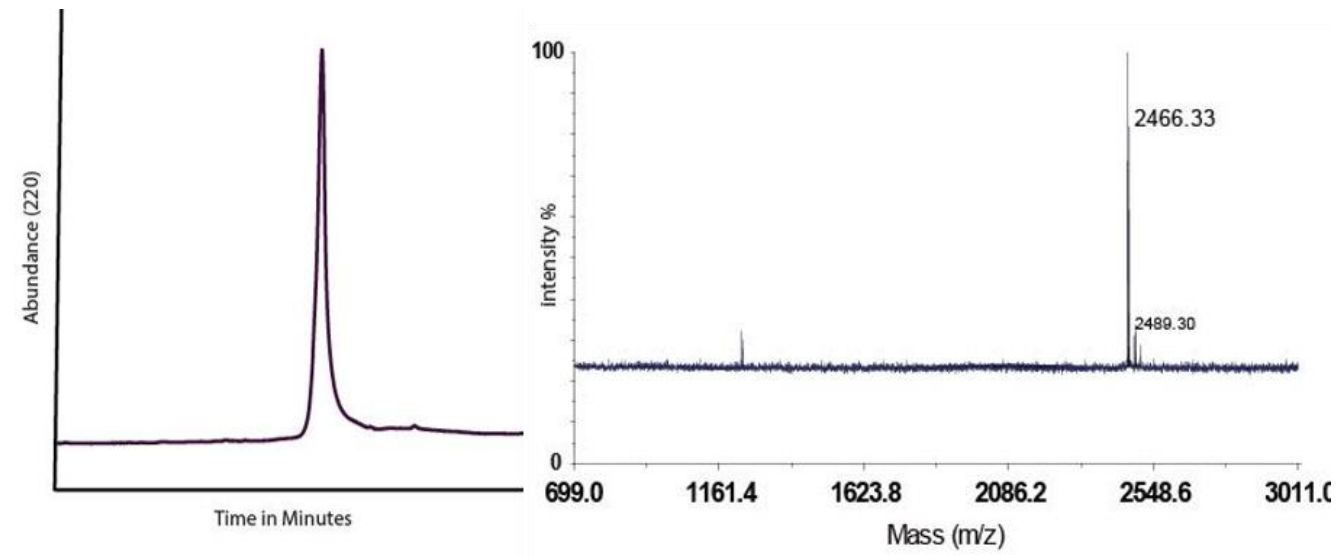

Figure 4.33 Analytical HPLC (left) and MALDI-TOF MS (right) of the Cys9, Orn10-teixobactin dimer 13b. Observed $\left[\mathrm{M}+\mathrm{H}^{+}\right]^{+}=2466.33$.

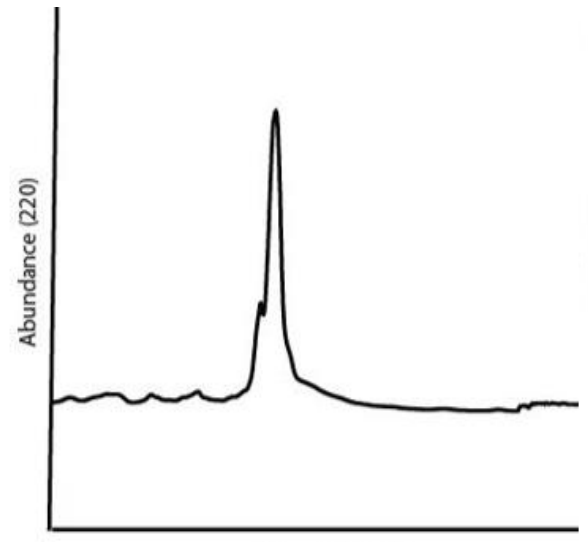

Time in Minutes

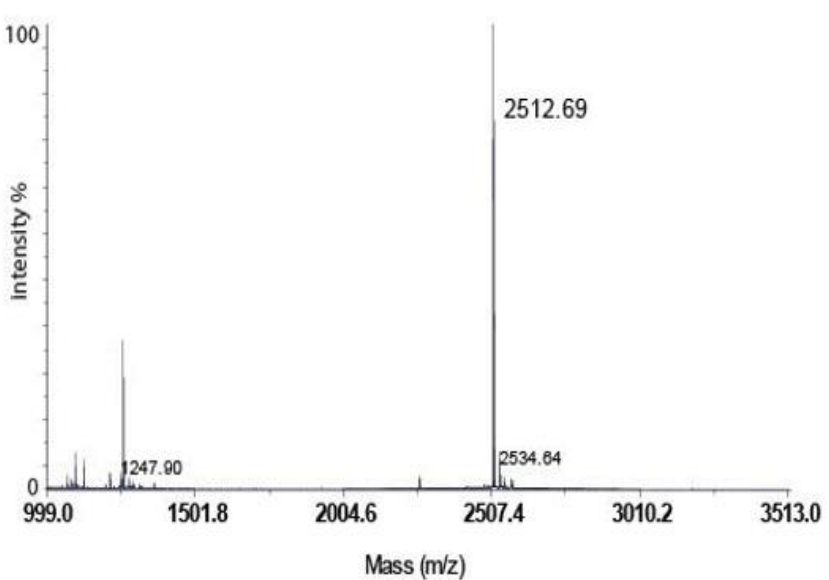

Figure 4.34 Analytical HPLC (left) and MALDI-TOF MS (right) of the Cys9,His10-teixobactin dimer 14b. Observed $\left[\mathrm{M}+\mathrm{H}^{+}\right]^{+}=2512.69$.

\subsubsection{Synthesis of teixobactin analogs via route $B$}

In this route, the full-length linear peptide is first assembled and then cyclization is done to form the cyclic depsipeptide ring (Figure 4.11). It is more convenient to make the enduracidine-substitution analogs since enduracidine or its substitutes are coupled as the last amino acid before the on-resin cyclization step. However, we found that the ester bond on the Thr side-chain was prone to $\beta$-elimination during repetitive exposure to piperidine (Figure 4.35). This is likely due to the more electron withdrawing nature of the azido-isoleucyl group 
which makes it a better leaving group than the Fmoc-isoleucyl group used at this position in route A. Because of this, about $50 \%$ of $\beta$-elimination product was observed after coupling the four amino acids after Ile6 on the main chain as shown by HPLC analysis of the microcleavage product (Figure 4.36). $\beta$-elimination

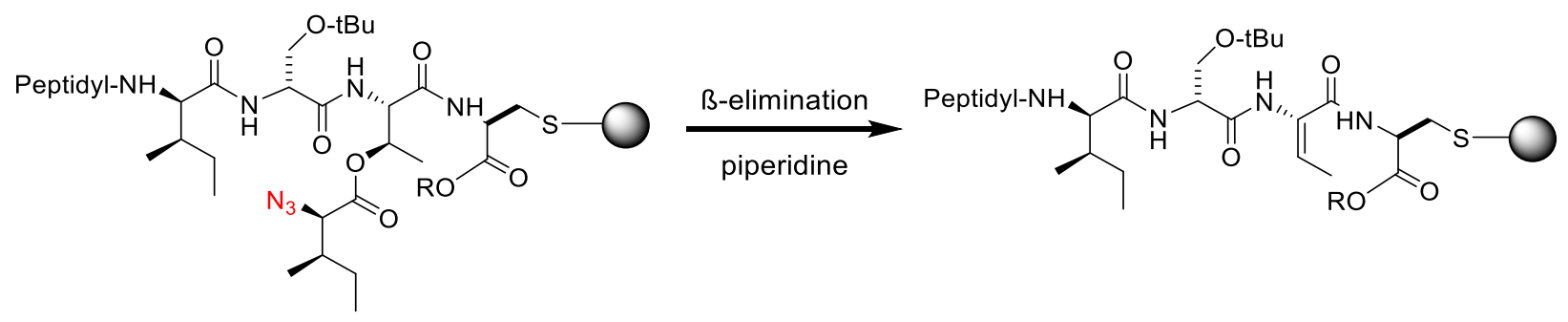

Figure 4.35 $\beta$-Elimination of the Thr ester during piperidine exposure.
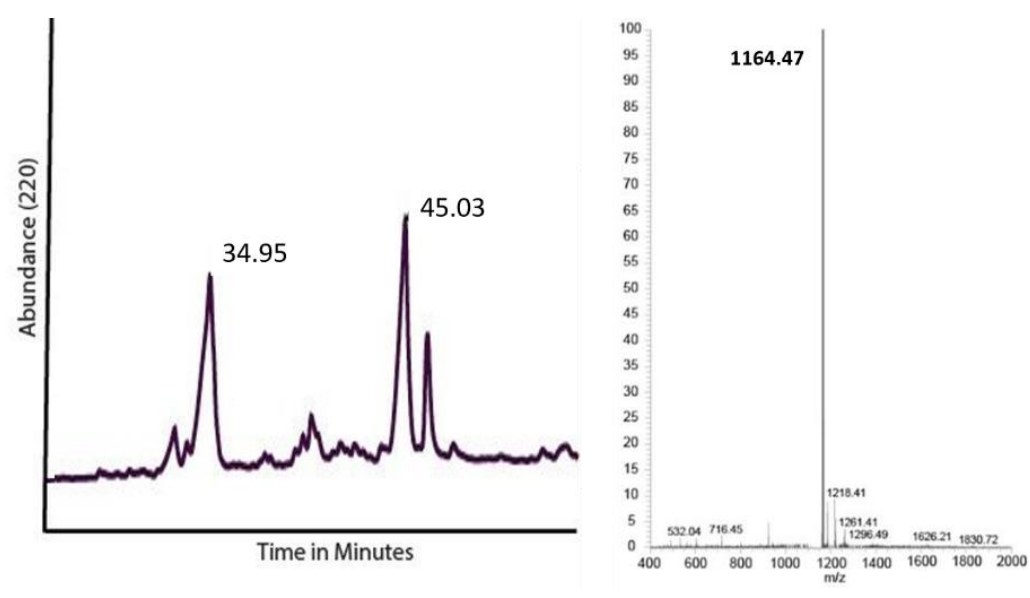

Figure 4.36 Analytical HPLC profile of NMe-D-Phe-Ile-Ser-D-Gln-D-allo-Ile-Ser-D-Thr(O-N3Ile)-Cys-OH (at $45.032 \mathrm{~min}$ ) after microcleavage and ESI-MS (right). The peak at $34.953 \mathrm{~min}$ is the $\beta$-elimination product, NMeD-Phe-Ile-Ser-D-Gln-D-allo-Ile-Ser-anhydroThr-Cys-OH, with a found $\left[\mathrm{M}+\mathrm{H}^{+}\right]^{+}=1007$.

So, although route B was developed to make the teixobactin analogs and despite its advantage in introducing substitutions at the crucial position 10, the overall yields were much lower than those obtained from route A. Therefore, synthetic route B was not pursued further for the synthesis of teixobactin analogs. 


\subsection{Antimicrobial activities of teixobactin analogs}

In this study, we prepared a number of teixobactin analogs in which the unusual amino acid L-allo-enduracidine at position 10 was replaced by other basic amino acids. The synthesis of L-allo-enduracidine is challenging, so we did not prepare the native teixobactin. All the analogs synthesized contain an amino-acid substitution at position 10 that has a varying-length side chain carrying a functional group with varying basicity. This provides an opportunity to investigate the structure-activity relationship of this position.

An arginine teixobactin analog was synthesized with L-arginine at position 10, and the remaining residues are the same as the natural teixobactin. Both enduracidine and arginine have a guanidine moiety, so arginine is the closest structural substitute of enduracidine except that Arg has a linear side chain whereas enduracidine has its guanidine group embedded in a 5-membered ring structure. Previous publications report the arginine analog to be one order of magnitude less active than teixobactin. Yang et al. ${ }^{105}$ have reported that the antibacterial activity of Lys-teixobactin is 2-4 times lower than arginine-teixobactin. Their study implies that guanidine is not mandatory to develop potent analogs. Therefore, different analogs were synthesized with other amino acid residues at this position. The antibacterial activity of the analogs was tested against several bacterial strains - the Gram-negative Escherichia Coli and two Gram-positive Enterococcal feacalis and Staphylococcus aureus, while vancomycin was used as a positive control. The antibacterial results, which were obtained from radial diffusion assays, are summarized in Table 4.2.

From Table 4.2, we can see that the enduracidine10-substituted analogs have comparable activities no matter the amino acid substitution is Arg, Lys, Orn or His with a guanidine, primary amine or imidazole functional group on the side chain of the amino acid. Therefore, the guanidinium group is not necessary for the activity at least in the nonenduracidine series of analogs. The cyclic guanidino group of enduracidine seems to be important in providing higher antibacterial activity as reported by others, but we did not make the native compound for comparison in our study. With respect to the length of the side chain, 
a comparison between Lys10-Teixobactin 12a and Orn10-Teixobactin 13a suggests that there is no significant effect of having a one-carbon longer or shorter side chain. Among the analogs synthesized, 11a, 12a, 13a and 14a are very similar to the natural teixobactin with the exception of residue at position 10. The synthetic scheme developed in this thesis allows the preparation of dimeric analogs of teixobactin through Cys oxidation. Most interestingly, the dimeric versions of the analogs are also very active, with some being more active than their monomeric counterparts. Notably, the dimeric form of Cys9,Arg10-teixobactin is significantly more active than the monomers.

Table 4.2 Antimicrobial activity of different teixobactin analogs.

\begin{tabular}{|l|c|c|c|}
\hline \multicolumn{4}{|c|}{ Minimum Inhibitory Concentration (MIC) $(\boldsymbol{\mu M})$} \\
\hline Peptide & $\begin{array}{l}\text { Staphylococcus } \\
\text { Aureus }\end{array}$ & $\begin{array}{l}\text { Escherichia } \\
\text { Coli }\end{array}$ & $\begin{array}{l}\text { Enterococcus } \\
\text { faecalis }\end{array}$ \\
\hline Cys9,Arg10-Teixobactin 11 & $9.9 \pm 0.8$ & $5 \pm 0.6$ & $6 \pm 0.8$ \\
\hline Arg10-Teixobactin 11a & $2.2 \pm 0.3$ & $2.3 \pm 0.3$ & $4 \pm 0.5$ \\
\hline Dimer of Cys9,Arg10-Teixobactin 11b & $0.52 \pm 0.4$ & $0.66 \pm 0.1$ & $0.8 \pm 0.1$ \\
\hline Cys9,Lys10-Teixobactin 12 & $3.3 \pm 0.2$ & $7.2 \pm 1$ & $1.9 \pm 0.3$ \\
\hline Lys10-Teixobactin 12a & $6 \pm 0.7$ & $1.46 \pm 0.3$ & $2 \pm 0.2$ \\
\hline Dimer of Cys9,Lys10-Teixobactin 12b & $1.5 \pm 0.2$ & $1.47 \pm 0.2$ & $0.64 \pm 0.2$ \\
\hline Cys9,Orn10-Teixobactin 13 & $6.2 \pm 0.8$ & $1.3 \pm 0.2$ & $1.3 \pm 0.3$ \\
\hline Orn10-Teixobactin 13a & $8.7 \pm 1$ & $2.9 \pm 0.4$ & $2.9 \pm 0.4$ \\
\hline Dimer of Cys9,Orn10-Teixobactin 13b & $1.8 \pm 0.3$ & $2.0 \pm 0.3$ & $2.0 \pm 0.5$ \\
\hline Cys9,His10-Teixobactin 14 & $>30$ & $11.1 \pm 1$ & $12.2 \pm 2$ \\
\hline His10-Teixobactin 14a & $4.96 \pm 0.5$ & $0.82 \pm 0.1$ & $1.16 \pm 0.2$ \\
\hline Dimer of Cys9,His10-Teixobactin 14b & $2.3 \pm 0.3$ & $1.30 \pm 0.5$ & $1.30 \pm 0.2$ \\
\hline Vancomycin & $8.4 \pm 1$ & $2 \pm 0.4$ & $1 \pm 0.3$ \\
\hline Escherichia Coli employed is a lab strain, and Staphylococcus aureus isolates exhibit MIC in an \\
\hline intermediate range similar to VISA. Enterococcal faecalis is ATCC 29212.
\end{tabular}




\subsection{Conclusion}

The objective of the project is to design a simple scheme for teixobactin synthesis. After numerous attempts, a robust methodology for the synthesis of teixobactin and its analogs have been developed. An important aspect of this methodology is the use of an orthogonal protecting group strategy. We realize that azide is a versatile precursor to a primary amine as it can be easily reduced with TCEP on solid phase. Therefore it is essentially an orthogonal protecting group for the amine because it is stable to the usual conditions of solid phase peptide synthesis. The use of azido-isoleucine indeed was one of the key elements to the overall success of our strategy.

The most remarkable feature of our strategy is the on-resin cyclization step that forms the depsitetrapeptide ring. This distinguishes our strategy from all the previously reported methods in which cyclization was done in solution on the protected full-length linear peptide. On-resin cyclization was made possible by using a Cys residue at the position of Ala9 in the cyclotetrapeptide ring and attaching the thiol group of Cys to a trityl resin. Because in our strategy everything is done on resin, we could take full advantage of the solid phase peptide synthesis methodology and no intermediate separation or purification steps were needed. This makes it easy to prepare the analogs of teixobactin as the pseudodilution effect avoids many of the inherent problems of solution cyclization reactions such as poor solubility of protected peptides and cyclo-oligomerization that often accompanies the formation of small rings. Our strategy allows a great degree of flexibility in adding many chemical moieties. The synthesis of teixobactin arginine analogs was achieved with an overall yield of $20 \%$. The analogs prepared have comparable antibacterial activities to those reported in the literature. In future work, we plan to conduct antimicrobial studies of the synthesized analogs on more bacterial strains. In the same time, native teixobactin and its analogs with substitutions at other positions and with truncated sequences will be prepared to derive a more comprehensive structureactivity relationship. 


\subsection{Materials and Method}

\subsubsection{Materials}

Benzotriazol-1-yl-oxytripyrrolidinophosphonium hexafluorophosphate (PyBOP) and 2-chloro-trityl chloride resin were purchased from GL Biochem (Shanghai). N,Ndiisopropylethylamine (DIEA), nickel acetate and sodium borohydride were purchased from Sigma-Aldrich (Singapore). Fmoc-Arg(pdf)-OH, Fmoc-Lys(Boc)-OH, Fmoc-Ser(tBu)-OH and Fmoc-Ile-OH were purchased from Novabiochem. Fmoc-D-Thr-OH, Fmoc-D-Ile-OH, Fmoc-D-allo-Ile-OH, and Fmoc-d-Gln(Trt)-OH were purchased from Chem-Impex International. Fmoc-N-Me-D-Phe-OH was purchased from Atomole. The unprotected isoleucine was purchased from GL Biochem to make azide isoleucine. $N, N$ dimethylformamide (DMF), dichloromethane (DCM), diethyl ether, and HPLC-grade acetonitrile (ACN) were purchased from Fisher. Trifluoroacetic acid (TFA) was purchased from Alfa Aesar (United Kingdom). Buffer A used for RP-HPLC was $0.045 \%$ and $\mathrm{ddH}_{2} \mathrm{O}$. Buffer B was $90 \% \mathrm{ACN}, 10 \% \mathrm{ddH}_{2} \mathrm{O}$ and $0.039 \%$ TFA. Triisopropylsilane (TRIS) was purchased from Alfa aesar. Ethane dithiol was obtained Fluka Chemical. Piperidine was obtained from Acrons. Reagent A for Ninhydrin test was prepared by dissolving $1.0 \mathrm{~g}$ of ninhydrin in $20 \mathrm{~mL}$ of $\mathrm{n}$-butanol. Reagent B for Ninhydrin test was prepared by dissolving 40 $\mathrm{g}$ of phenol in $20 \mathrm{~mL}$ of $\mathrm{n}$-butanol. Reagent $\mathrm{C}$ for Ninhydrin test was prepared by dissolving $16.5 \mathrm{mg}$ of $\mathrm{KCN}$ in $25 \mathrm{~mL}$ of distilled water, after which $1.0 \mathrm{~mL}$ of the solution was diluted with $49 \mathrm{~mL}$ of pyridine.

The instruments used are the same as in the previous chapters.

\subsubsection{Methods}

\subsubsection{Preparation of Fmoc-Cys-OH from Fmoc-Cys(Trt)-OH}

A suspension made of $100 \mathrm{ml}$ DCM containing 2g (3.42 mmol) of Fmoc-Cys(Trt)-OH was acidified with $4 \mathrm{ml}$ of TFA. Once TFA is added, the mixture changed to a dark brown solution. The mixture was kept on a stirrer for $5 \mathrm{~h}$. Then $4 \mathrm{ml}$ of triisopropylsilane (TIS) was 
added and the mixture became light yellow. The reaction mixture was shaken for 30 more min. Then 2-3 drops of TFA were added and no change in color was observed. Then it was kept on a stirrer for one more hour. An additional $0.5 \mathrm{ml}$ of TIS was added and stirred for some more time. The light yellow color did not change. After evaporating the solvents, a solid was obtained. The residue was washed with hexane $(2 \mathrm{X})$ and the solvent decanted. Final washing was conducted with DCM and dried on the rotary evaporator. To remove remaining solvents, the product obtained was lyophilized.

\subsubsection{Loading Fmoc-Cys-OH to 2-Cl-trityl chloride resin and protection of $\mathrm{COOH}$}

$2.5 \mathrm{~g}$ of 2-cholotrityl chloride resin with a substitution of $1 \mathrm{~g}=1.07 \mathrm{mmol} / \mathrm{g}$ was taken. It was swollen with dry DCM, then treated with 30\% TFA for $2 \mathrm{~min}$. The resin turned dark red and more swollen and it was kept shaking for another 10 minutes. After that, it was washed with dry DCM. The color remained dark red. Then 1.2 equivalents of Fmoc-Cys-OH dissolved in DCM was added to the resin. After shaking for $1 \mathrm{~h}$, a small amount of DMF was added to the reaction mixture. During the reaction one can observe that dark-color resin changed to light brown yellow. Then it was washed with DCM 3X and DMF 3X. The final product was 2. The carboxyl group of Cys was then protected with trityl or 2-chlorotrityl group. The trityl chloride or 2-chlorotrityl chloride (3 equiv) in dry DCM and DIEA (6 equiv) were added to the resin. The reaction mixture was kept on a shaker for $12 \mathrm{~h}$. The resin was washed with DCM (3X) and DMF (3X), and with DCM (2X) to afford 3.

\subsubsection{Azido-isoleucine}

$\mathrm{NaN}_{3}(5.34 \mathrm{~g}, 82.35 \mathrm{mmol})$ was dissolved in $\mathrm{H}_{2} \mathrm{O}(13.5 \mathrm{~mL})$ and mixed with DCM (22.5 mL). The mixture was cooled down to $0{ }^{\circ} \mathrm{C}$. Then $\mathrm{Tf}_{2} \mathrm{O}(2.79 \mathrm{~mL}, 16.65 \mathrm{mmol})$ was added over a period of $5 \mathrm{~min}$, and the reaction mixture was kept on a stirrer for $2 \mathrm{~h}$. The mixture was placed in a separatory funnel and the organic phase was removed. The aqueous layer was extracted with DCM $(2 \times 10 \mathrm{~mL})$. The pooled organic phase, containing the triflyl azide, was washed once with a saturated aqueous solution of $\mathrm{NaHCO}_{3}$, and used without further purification. 
The free isoleucine (1.098 g, $8.37 \mathrm{mmol}), \mathrm{K}_{2} \mathrm{CO}_{3}(1.732 \mathrm{~g}, 12.57 \mathrm{mmol}), \mathrm{CuSO}_{4} 5 \mathrm{H}_{2} \mathrm{O}$ (21 mg, $83.7 \mu \mathrm{mol}$ ) were dissolved in distilled $\mathrm{H}_{2} \mathrm{O}(27 \mathrm{~mL})$ and $\mathrm{MeOH}(54 \mathrm{~mL})$. The above triflyl azide in DCM was added, and the mixture was stirred overnight. The organic solvents were removed under vacuum, and the aqueous phase was diluted with $\mathrm{H}_{2} \mathrm{O}(100 \mathrm{~mL})$. This aqueous mixture was acidified to $\mathrm{pH} 6$ with concentrated $\mathrm{HCl}$ and diluted with $0.25 \mathrm{M} \mathrm{pH} 6.2$ phosphate buffer $(100 \mathrm{~mL})$ and extracted with Ethylacetate $(4 \times 100 \mathrm{~mL})$ to remove the sulphonamide byproduct. The aqueous phase was acidified to $\mathrm{pH} 2$ with concentrated $\mathrm{HCl}$. The product was obtained by extraction with Ethyl acetate $(3 \mathrm{x} 100 \mathrm{~mL})$. The organic extracts were combined, dried over anhydrous $\mathrm{Na}_{2} \mathrm{SO}_{4}$, filtered and the solvent was removed under vacuum to give $1.170 \mathrm{~g}$ of pure azido-isoleucine (yield $89 \%$ ) as a pale oil.

\subsubsection{Esterification}

Esterification conditions corresponding to respective serial no in Table 4.1

1. The peptide Boc-dF-I-S(tBu)-dQ(Trt)-dI-I-S(tBu)-dT-C(2-Cl-trityl resin)-Trt was prepared by standard SPPS and the Boc group was added using $\mathrm{Boc}_{2} \mathrm{O}$ after Fmoc on d-Phe was removed. The peptidyl resin was subjected to esterification using Fmoc-Ile-OH (4 equiv), chloro-N,N,N',N'-tetramethylformamidinium hexafluorophosphate (4 equiv), DMAP (0.4 equiv) in dry DCM. The reaction was kept for 18 hours, and approximately $25 \%$ of the required product was observed after analyzing the microcleavage product.

2. The peptide Boc-S(tBu)-dT-C(2-Cl-trityl resin)-Trt was subjected to esterification using $\mathrm{N}_{3}$-Ile-OH ( 5 equiv), DIC (5 equiv), DMAP ( 0.5 equiv) in DCM:DMF (8:2) mixture for $18 \mathrm{~h}$ at $37^{\circ} \mathrm{C}$. Then 5 equiv DIC were added again and the reaction was kept for another $18 \mathrm{~h}$. Then the reaction was analyzed after microcleavage, which showed that more than $90 \%$ of the peptide was esterified and no starting material was observed.

3. The peptide $\mathrm{N}_{3}-\mathbf{I}-\mathbf{S}-\mathbf{d} \mathbf{T}(\mathrm{OH})-\mathbf{C}(2-\mathrm{Cl}$-trityl resin)-Trt was subjected to the esterification condition with 10 equivalents Fmoc-ile-OH, 10 equivalents DIC, 1 equivalent 
DMAP in DCM:DMF(8:2). It was kept on a shaker for 3 hours and the required product was obtained with more than $90 \%$ conversion according to microcleavage analysis.

4. $\mathrm{N}_{3}-\mathbf{I}-\mathbf{S}-\mathbf{d T}(\mathrm{OH})-\mathbf{C}(2-\mathrm{Cl}-$ trityl resin)-Trt was subjected to esterification with 10 equivalent Fmoc-ile-OH, 5 equivalent DIC, 1 equivalent DMAP in $\operatorname{DCM}: \operatorname{DMF}(8: 2)$. It was kept shaking for 3 hours and the required product was observed with more than $90 \%$ conversion.

5. N3-I-S-dT(OH)- C(2-Cl-trityl resin)-2Cl-Trt was subjected to esterification with 8 equivalent Fmoc-ile-OH, 8 equivalent DIC, 0.8 equivalent DMAP in DCM:DMF(8:2). It was kept on a shaker for 3 hours and the required product was obtained with more than $90 \%$ conversion.

6. Fmoc-S-dT(OH)-C(2-Cl-trityl resin)-2Cl-Trt was esterified with 5 equivalent azido-isoleucine, 5 equivalent DIC and 0.5 equivalent DMAP in DCM:DMF (8:2). Esterification was complete in around $6 \mathrm{~h}$ with more than 90\% conversion from HPLC analysis of the microcleavage product.

\subsubsection{Synthesis of the intermediate resin-bound peptides}

\section{i) Peptide-resin $4 a, b$}

The cysteine linked resin 3a,b was deprotected with 20\% piperidine in DMF for 5 min and 20 min. Using standard SPPS protocols, Fmoc-D-Thr(OH)-OH and Fmoc-Ser(Trt)-OH were coupled.

\section{ii) Peptide-resin 5a,b}

Peptide-resin 4a,b was deprotected with $20 \%$ piperidine in DMF. Azido-isoleucine was coupled using the normal coupling protocol.

\section{iii) Peptide-rein 6a,b}

The esterification was conducted using the conditions mentioned above in section 4.3.2.4. 


\section{iv) Peptide-resin 7a,b}

After deprotecting Fmoc, Fmoc-Arg(Pbf)-OH was coupled to peptide-resin 6 using standard SPPS protocols.

\section{v) Peptide 8}

Before starting cyclization reaction, the peptide was deprotected with $20 \%$ piperidine after exposing it for $5 \mathrm{~min}$ followed by 20 minutes. Then Kaiser test was done to confirm Fmoc deprotection. To deprotect the trityl (or 2-Cl-trityl) group from the carboxyl group of Cys, 0.5\% TFA solution in DCM was added to the resin and the mixture was shaken for approximately 3 minutes. After draining, the treatment with $0.5 \%$ TFA was repeated two more times, followed by a short exposure to $0.75 \%$ TFA in DCM for a few seconds. After that, the resin was washed $4 \mathrm{X}$ DCM and then $4 \mathrm{X} \mathrm{DMF}$.

\section{vi) Peptide-resin 9}

For on-resin cyclization, about 3 equivalents of PyBOP and 6 equivalents DIEA were in DCM/DMF were poured into the reaction vessel. The reaction vessel was kept on a shaker for at about $6 \mathrm{~h}$. Microcleavage was conducted on a small amount of resin beads at different intervals to assess the cyclization efficiency. After 6 hours, the resin was washed with DCM and DMF.

\section{vii) Peptide-resin 10}

After cyclization, $0.25 \mathrm{M}$ TCEP solution in DMF with $2.5 \% \mathrm{H}_{2} \mathrm{O}$ was added to the reaction vessel containing peptide-resin 9 and it was shaken for $4 \mathrm{~h}$, which was more than enough for the reduction of azide which is usually quite.

\section{viii) Peptide-resin 11}

Once 10 was obtained, the remaining amino acids were coupled using the standard method of solid phase peptide synthesis, followed by the final cleavage to afford $\mathbf{1 1}$.

\subsubsection{Desulfurization}

To prepare Raney nickel, nickel acetate (3.0 g) was dissolved in $75 \mathrm{~mL}$ of $\mathrm{H}_{2} \mathrm{O}$, and then sodium borohydride $(0.5 \mathrm{~g})$ was slowly added with stirring. ${ }^{114}$ The black amorphous 
nickel precipitate was filtered and washed with distilled water until the washing became neutral in $\mathrm{pH}$. The nickel was then transferred into a closed bottle and stored in $\mathrm{H}_{2} \mathrm{O}$ at $4{ }^{\circ} \mathrm{C}$. Peptide desulfurization was carried out with two different conditions: in 20\% acidic acid or in $6 \mathrm{M}$ guanidine hydrochloride solution.

(i) In $20 \%$ acetic acid. The lyophilized peptide 11 was solubilized in $20 \%$ aqueous acetic acid. The above prepared Raney nickel was added to approximately $10 \%$ or more of the reaction volume. The mixture was shaken at $37{ }^{\circ} \mathrm{C}$ for $3-5 \mathrm{~h}$. Desulfurization reaction was monitored by analytical HPLC and ESI-MS. The reaction mixture was then filtered and the filtrate was injected onto the HPLC column for purification and the pure fraction were lyophilized to give 11a in powder form.

ii) In $6 \mathrm{M}$ guanidine hydrochloride solution. The peptide 11 was dissolved in $6.0 \mathrm{M}$ guanidine. $\mathrm{HCl}$ solution $(0.1 \mathrm{M}$ phosphate buffer, $\mathrm{pH} 5.8)$. The catalyst Raney nickel used was at least $10 \%$ of the reaction volume. An excess amount of TCEP was added to keep the peptide in its reduced form. The mixture was shaken at $37{ }^{\circ} \mathrm{C}$ for $5 \mathrm{~h}$. Desulfurization reaction was monitored by analytical HPLC and ESI-MS. The reaction mixture was then filtered and the filtrate was acidified with TFA and loaded to HPLC column for purification and the pure fraction were lyophilized to give 11a in powder form.

\subsubsection{Synthesis of Peptide 12, 13 and 14}

The syntheses of 12,13 and 14 were similarly done as for peptide 11 except that FmocLys(Boc)-OH, Fmoc-Orn(Boc)-OH and Fmoc-His(trt)-OH were coupled to the peptide $6 \mathbf{b}$.

Desulfurization conditions mentioned above were employed for conversion of $\mathbf{1 2}$ to 12a, 13 to $13 a$ and 14 to $14 a$.

\subsubsection{Radial diffusion assay}

Radial diffusion assays were conducted in a similar way as described in section 2.3.8. 


\section{CHAPTER 5 \\ GENERAL CONCLUSION}

The aim of the work is to develop antibacterial agents that can be used to fight drugresistant strains. The work is focused on developing peptide-based antibacterials that particularly target the bacterial membrane and the cell-wall components. Agents that act on these targets are known to impose high barriers for bacterial resistance. The antibacterials studied in this thesis include AMPs which are considered as next-generation antibiotics, vancomycin - one of the most potent glycopeptide antibiotic, and teixobactin - a recently discovered antibiotic that can significantly help in preventing bacterial drug resistance. New chemical methods are introduced that facilitate the design and development of novel analogs of known compounds with proven antibacterial activities.

Chapter 2 deals with dimerization of antimicrobial peptides (AMPs) in the hope to improve their antibacterial activity and stability. The presence of two copies of an antimicrobial peptide in the dimer can incur higher concentration of membrane disturbing entities against bacterial phospholipid. Traditionally, peptide dimers were synthesized on a bivalent lysine core in solid phase peptide synthesis, i.e., amino acids were coupled step wisely to the $\alpha$ and $\varepsilon$ amines of Lys using standard SPPS procedures. In chapter 2, two different dimerization methods are utilized: the traditional SPPS method just mentioned above and an unconventional convergent method that is based on the thiol alkylation reaction. The convergent approach is more effective and addresses the limitations of solid phase peptide synthesis.

The consequences of dimerization of two types of AMPs (cationic hydrophilic AMPs and hydrophobic AMPs) are strikingly different. The highly cationic V2 peptide derived from human beta defensin-3 is a hydrophilic antimicrobial peptide. V2 peptide dimers exhibit greater antibacterial property then the V2 monomer. On the other hand, DFTamP1-p is an 
artificially designed hydrophobic $\alpha$-helical AMP; interestingly, dimers of DFTamP1-p show poorer antibacterial activity than the monomer. So, dimerization is counter-productive for the hydrophobic and less cationic AMPs like DFTamP1-p. Therefore, a better understanding of the effect of dimerization with respect to the mechanisms of action is needed for the rational design of dimeric AMPs.

Chapter 3 describes a simple and efficient single-step strategy to synthesize potent analogs of vancomycin. The strategy involves modification of vancomycin through its carboxylic group by reacting with amine compounds. The strategy can also be extended to other members of glycopeptide with a free carboxyl group. Vancomycin is one of the most potent antibiotics among glycopeptide antibiotics. Oritavancin, Talbavancin, and dalbavancin are analogs of vancomycin, ${ }^{28}$ now approved by FDA for treating drug-resistant bacteria. The strategy of reacting vancomycin with diamines seems to be promising to increase its antibacterial potency. The strategy can also be used to develop vancomycin dimers, or conjugate it with other antimicrobial peptides.

Results obtained in Chapter 3 have further corroborated previous findings that the presence of an additional amine on a hydrocarbon chain at the C-terminal of glycopeptide antibiotics can improve the antibacterial activity. The method of carboxamide formation offers numerous possibilities to develop potent vancomycin analogs. However, to complete the study, antimicrobial testing against vancomycin-resistant strains will be significant. Vancomycin is lipid II inhibitor, and its carboxamide amine analogs act on at least two different sites of the target. As a part of our future work, we intend to synthesize analog with 4-chlorobiphenyl moieties attached at the sugar of carboxamide vancomycin analogs. Attaching multiple moieties on vancomycin may impart three different modes of actions to vancomycin and hence increase its antibacterial potential against drug resistant strains.

In chapter 4, an efficient and robust synthetic scheme is reported for the synthesis of teixobactin analogs. Teixobactin is a 11-mer peptide that contains a cyclic depsipeptide ring at its C-terminal end. In our study, a simple solid phase synthesis scheme for teixobactin 
analogs has been developed which offers significant advantages over the reported methods. We have established an efficient O-acylation protocol to form the depsi ester bond on D-Thr8 and a robust on-resin cyclization procedure. Through the use of a cysteine residue for attaching to the solid phase via the thiol group, all the crucial synthetic steps can be carried out on a solid phase. Our all-SPPS scheme provides a great degree of flexibility in making various substitutions at different positions of teixobactin, allowing high-throughput synthesis of analogs with enormous diversities. Due to time constraints, we only focused on substitutions of enduracidine at position 10 of the molecule. We have shown that enduracidine-10 substitution analogs such as Arg10-teixobactin have comparable activities as those reported in the literature. As the immediate products of our SPPS scheme contain a Cys residue at position 10, dimeric analogs can be easily obtained by oxidation of the cysteinyl thiol. It is shown that the S-S linked dimers are also potent antibacterial agents. We anticipate that our robust SPPS scheme will accelerate the discovery and development of new cyclodepsipeptide antibiotics with much better antibacterial properties in the future. 


\section{REFERENCES}

1. Lewis, K., Antibiotics: Recover the lost art of drug discovery. Nature 2012, 485 (7399), 43940.

2. Gale, E. F., Correlation between penicillin resistance and assimilation affinity in Staphylococcus aureus. Nature 1947, 160 (4064), 407.

3. Palumbi, S. R., Humans as the world's greatest evolutionary force. Science 2001, 293 (5536), 1786-1790.

4. Harbarth, S.; Samore, M. H., Antimicrobial resistance determinants and future control. Emerg Infect Dis 2005, 11 (6), 794-801.

5. Aminov, R. I.; Mackie, R. I., Evolution and ecology of antibiotic resistance genes. FEMS microbiology letters 2007, 271 (2), 147-161.

6. Arias, C. A.; Murray, B. E., Emergence and management of drug-resistant enterococcal infections. Expert review of anti-infective therapy 2008, 6 (5), 637-655.

7. Hiramatsu, K., Vancomycin-resistant Staphylococcus aureus: a new model of antibiotic resistance. The Lancet infectious diseases 2001, 1 (3), 147-155.

8. Clatworthy, A. E.; Pierson, E.; Hung, D. T., Targeting virulence: a new paradigm for antimicrobial therapy. Nature chemical biology 2007, 3 (9), 541-548.

9. Dheda, K.; Gumbo, T.; Gandhi, N. R.; Murray, M.; Theron, G.; Udwadia, Z.; Migliori, G. B.; Warren, R., Global control of tuberculosis: from extensively drug-resistant to untreatable tuberculosis. The Lancet. Respiratory medicine 2014, 2 (4), 321-38.

10. Gandhi, N. R.; Nunn, P.; Dheda, K.; Schaaf, H. S.; Zignol, M.; van Soolingen, D.; Jensen, P.; Bayona, J., Multidrug-resistant and extensively drug-resistant tuberculosis: a threat to global control of tuberculosis. Lancet (London, England) 2010, 375 (9728), 1830-43.

11. WHO Global Tuberculosis Report; WHO: 2015.

12. Shah, N. S.; Wright, A.; Bai, G.-H.; Barrera, L.; Boulahbal, F.; Drobniewski, F.; Gilpin, C.; Havelkov, M.; Lepe, R.; Lumb, R., Worldwide emergence of extensively drug-resistant tuberculosis. 2007.

13. Mitnick, C. D.; Shin, S. S.; Seung, K. J.; Rich, M. L.; Atwood, S. S.; Furin, J. J.; Fitzmaurice, G. M.; Alcantara Viru, F. A.; Appleton, S. C.; Bayona, J. N., Comprehensive treatment of extensively drug-resistant tuberculosis. New England Journal of Medicine 2008, 359 (6), 563-574.

14. Parida, S. K.; Axelsson-Robertson, R.; Rao, M. V.; Singh, N.; Master, I.; Lutckii, A.; Keshavjee, S.; Andersson, J.; Zumla, A.; Maeurer, M., Totally drug-resistant tuberculosis and adjunct therapies. Journal of internal medicine 2015, 277 (4), 388-405.

15. Barbosa, T. M.; Levy, S. B., The impact of antibiotic use on resistance development and persistence. Drug resistance updates 2000, 3 (5), 303-311.

16. Harvey, A. L.; Edrada-Ebel, R.; Quinn, R. J., The re-emergence of natural products for drug discovery in the genomics era. Nature reviews. Drug discovery 2015, 14 (2), 111-29.

17. Wang, G.; Li, X.; Wang, Z., APD2: the updated antimicrobial peptide database and its application in peptide design. Nucleic acids research 2009, 37 (suppl 1), D933-D937.

18. Scott, M. G.; Yan, H.; Hancock, R. E., Biological properties of structurally related $\alpha$-helical cationic antimicrobial peptides. Infection and immunity 1999, 67 (4), 2005-2009. 
19. Steckbeck, J. D.; Deslouches, B.; Montelaro, R. C., Antimicrobial peptides: new drugs for bad bugs? Taylor \& Francis: 2014.

20. Fernandes, P., Antibacterial discovery and development-the failure of success? Nature biotechnology 2006, 24 (12), 1497-1503.

21. Tillotson, G. S., Quinolones: structure-activity relationships and future predictions. Journal of medical microbiology 1996, 44 (5), 320-4.

22. Arias, C. A.; Murray, B. E., A new antibiotic and the evolution of resistance. The New England journal of medicine 2015, 372 (12), 1168-70.

23. Ling, L. L.; Schneider, T.; Peoples, A. J.; Spoering, A. L.; Engels, I.; Conlon, B. P.; Mueller, A.; Schaberle, T. F.; Hughes, D. E.; Epstein, S.; Jones, M.; Lazarides, L.; Steadman, V. A.; Cohen, D. R.; Felix, C. R.; Fetterman, K. A.; Millett, W. P.; Nitti, A. G.; Zullo, A. M.; Chen, C.; Lewis, K., A new antibiotic kills pathogens without detectable resistance. Nature 2015, 517 (7535), 4559.

24. Sivanathan, S.; Scherkenbeck, J., Cyclodepsipeptides: A rich source of biologically active compounds for drug research. Molecules 2014, 19 (8), 12368-12420.

25. López-Macià, À.; Jiménez, J. C.; Royo, M.; Giralt, E.; Albericio, F., Synthesis and Structure Determination of Kahalalide F1, 2. Journal of the American Chemical Society 2001, 123 (46), 11398-11401.

26. Arbeit, R. D.; Maki, D.; Tally, F. P.; Campanaro, E.; Eisenstein, B. I., The safety and efficacy of daptomycin for the treatment of complicated skin and skin-structure infections. Clinical Infectious Diseases 2004, 38 (12), 1673-1681.

27. Fox, J. L., Antimicrobial peptides stage a comeback. Nature biotechnology 2013, 31 (5), 379.

28. Breukink, E.; de Kruijff, B., Lipid II as a target for antibiotics. Nature reviews Drug discovery 2006, 5 (4), 321-323.

29. Dubos, R. J., Studies on a bactericidal agent extracted from a soil bacillus: I. Preparation of the agent. Its activity in vitro. The Journal of experimental medicine 1939, 70 (1), 1.

30. Jago, W.; Jago, W.; Allen, W., Toxic action of wheat flour to brewer's yeast. Industrial Fermentations 1926, 128-167.

31. OHTANI, K.; OKADA, T.; YOSHIZUMI, H.; KAGAMIYAMA, H., Complete primary structures of two subunits of purothionin $A$, a lethal protein for brewer's yeast from wheat flour. Journal of biochemistry 1977, 82 (3), 753-767.

32. Zasloff, M., Antimicrobial peptides of multicellular organisms. Nature 2002, 415 (6870), 389 395.

33. Hancock, R. E., Cationic peptides: effectors in innate immunity and novel antimicrobials. The Lancet infectious diseases 2001, 1 (3), 156-164.

34. Hancock, R. E.; Sahl, H.-G., Antimicrobial and host-defense peptides as new anti-infective therapeutic strategies. Nature biotechnology 2006, 24 (12), 1551-1557.

35. Tossi, A.; Sandri, L., Molecular diversity in gene-encoded, cationic antimicrobial polypeptides. Current pharmaceutical design 2002, 8 (9), 743-761.

36. Fjell, C. D.; Hiss, J. A.; Hancock, R. E.; Schneider, G., Designing antimicrobial peptides: form follows function. Nature reviews Drug discovery 2012, 11 (1), 37-51.

37. Howerton, A.; Patra, M.; Abel-Santos, E., A new strategy for the prevention of Clostridium difficile infection. The Journal of infectious diseases 2013, 207 (10), 1498-504.

38. Giuliani, A.; Pirri, G.; Nicoletto, S., Antimicrobial peptides: an overview of a promising class of therapeutics. Open Life Sciences 2007, 2 (1), 1-33. 
39. Maccari, G.; Di Luca, M.; Nifosi, R.; Cardarelli, F.; Signore, G.; Boccardi, C.; Bifone, A., Antimicrobial peptides design by evolutionary multiobjective optimization. PLoS computational biology 2013, 9 (9), e1003212.

40. Mishra, B.; Wang, G., Ab initio design of potent anti-MRSA peptides based on database filtering technology. Journal of the American Chemical Society 2012, 134 (30), 12426-12429.

41. Tencza, S. B.; Creighton, D. J.; Yuan, T.; Vogel, H. J.; Montelaro, R. C.; Mietzner, T. A., Lentivirus-derived antimicrobial peptides: increased potency by sequence engineering and dimerization. Journal of Antimicrobial Chemotherapy 1999, 44 (1), 33-41.

42. Tam, J. P.; Lu, Y. A.; Yang, J. L., Antimicrobial dendrimeric peptides. European Journal of Biochemistry 2002, 269 (3), 923-932.

43. Jad, Y. E.; Acosta, G. A.; Naicker, T.; Ramtahal, M.; El-Faham, A.; Govender, T.; Kruger, H. G.; de la Torre, B. G.; Albericio, F., Synthesis and Biological Evaluation of a Teixobactin Analogue. Organic letters 2015, 17 (24), 6182-5.

44. Bracci, L.; Lozzi, L.; Pini, A.; Lelli, B.; Falciani, C.; Niccolai, N.; Bernini, A.; Spreafico, A.; Soldani, P.; Neri, P., A branched peptide mimotope of the nicotinic receptor binding site is a potent synthetic antidote against the snake neurotoxin $\alpha$-bungarotoxin. Biochemistry 2002, 41 (32), 10194-10199.

45. Bracci, L.; Falciani, C.; Lelli, B.; Lozzi, L.; Runci, Y.; Pini, A.; De Montis, M. G.; Tagliamonte, A.; Neri, P., Synthetic peptides in the form of dendrimers become resistant to protease activity. Journal of Biological Chemistry 2003, 278 (47), 46590-46595.

46. Sahly, H.; Schubert, S.; Harder, J.; Rautenberg, P.; Ullmann, U.; Schröder, J.; Podschun, R., Burkholderia is highly resistant to human Beta-defensin 3. Antimicrobial agents and chemotherapy 2003, 47 (5), 1739-1741.

47. Hultmark, D.; STEINER, H.; Rasmuson, T.; Boman, H. G., Insect immunity. Purification and properties of three inducible bactericidal proteins from hemolymph of immunized pupae of Hyalophora cecropia. European Journal of Biochemistry 1980, 106 (1), 7-16.

48. Schibli, D. J.; Hunter, H. N.; Aseyev, V.; Starner, T. D.; Wiencek, J. M.; McCray, P. B., Jr.; Tack, B. F.; Vogel, H. J., The solution structures of the human beta-defensins lead to a better understanding of the potent bactericidal activity of HBD3 against Staphylococcus aureus. The Journal of biological chemistry 2002, 277 (10), 8279-89.

49. Sahl, H.-G.; Pag, U.; Bonness, S.; Wagner, S.; Antcheva, N.; Tossi, A., Mammalian defensins: structures and mechanism of antibiotic activity. Journal of leukocyte biology 2005, 77 (4), 466475.

50. Klüver, E.; Schulz-Maronde, S.; Scheid, S.; Meyer, B.; Forssmann, W.-G.; Adermann, K., Structure-activity relation of human $\beta$-defensin 3: influence of disulfide bonds and cysteine substitution on antimicrobial activity and cytotoxicity. Biochemistry 2005, 44 (28), 9804-9816.

51. Joly, S.; Maze, C.; McCray, P. B.; Guthmiller, J. M., Human $\beta$-defensins 2 and 3 demonstrate strain-selective activity against oral microorganisms. Journal of clinical microbiology 2004, 42 (3), 1024-1029.

52. Maisetta, G.; Batoni, G.; Esin, S.; Luperini, F.; Pardini, M.; Bottai, D.; Florio, W.; Giuca, M. R.; Gabriele, M.; Campa, M., Activity of human $\beta$-defensin 3 alone or combined with other antimicrobial agents against oral bacteria. Antimicrobial agents and chemotherapy 2003, 47 (10), 3349-3351. 
53. Maisetta, G.; Batoni, G.; Esin, S.; Florio, W.; Bottai, D.; Favilli, F.; Campa, M., In vitro bactericidal activity of human $\beta$-defensin 3 against multidrug-resistant nosocomial strains. Antimicrobial agents and chemotherapy 2006, 50 (2), 806-809.

54. Silhavy, T. J.; Kahne, D.; Walker, S., The bacterial cell envelope. Cold Spring Harbor perspectives in biology 2010, 2 (5), a000414.

55. Wu, Z.; Hoover, D. M.; Yang, D.; Boulègue, C.; Santamaria, F.; Oppenheim, J. J.; Lubkowski, J.; Lu, W., Engineering disulfide bridges to dissect antimicrobial and chemotactic activities of human $\beta$-defensin 3. Proceedings of the National Academy of Sciences 2003, 100 (15), 88808885.

56. Midorikawa, K.; Ouhara, K.; Komatsuzawa, H.; Kawai, T.; Yamada, S.; Fujiwara, T.; Yamazaki, K.; Sayama, K.; Taubman, M. A.; Kurihara, H., Staphylococcus aureus susceptibility to innate antimicrobial peptides, $\beta$-defensins and CAP18, expressed by human keratinocytes. Infection and immunity 2003, 71 (7), 3730-3739.

57. Nishi, H.; Komatsuzawa, H.; Fujiwara, T.; McCallum, N.; Sugai, M., Reduced content of lysylphosphatidylglycerol in the cytoplasmic membrane affects susceptibility to moenomycin, as well as vancomycin, gentamicin, and antimicrobial peptides, in Staphylococcus aureus. Antimicrobial agents and chemotherapy 2004, 48 (12), 4800-4807.

58. Peschel, A.; Jack, R. W.; Otto, M.; Collins, L. V.; Staubitz, P.; Nicholson, G.; Kalbacher, H.; Nieuwenhuizen, W. F.; Jung, G.; Tarkowski, A., Staphylococcus aureus resistance to human defensins and evasion of neutrophil killing via the novel virulence factor MprF is based on modification of membrane lipids with I-lysine. The Journal of experimental medicine 2001, 193 (9), 1067-1076.

59. Zhou, L.; Liu, S.; Chen, L.; Li, J.; Ong, L.; Guo, L.; Wohland, T.; Tang, C.; Lakshminarayanan, R.; Mavinahalli, J., The structural parameters for antimicrobial activity, human epithelial cell cytotoxicity and killing mechanism of synthetic monomer and dimer analogues derived from hBD3 C-terminal region. Amino acids 2011, 40 (1), 123-133.

60. Ramamoorthy, A.; Thennarasu, S.; Lee, D.-K.; Tan, A.; Maloy, L., Solid-state NMR investigation of the membrane-disrupting mechanism of antimicrobial peptides MSI-78 and MSI-594 derived from magainin 2 and melittin. Biophysical journal 2006, 91 (1), 206-216.

61. Jang, W. S.; Kim, C. H.; Kim, K. N.; Park, S. Y.; Lee, J. H.; Son, S. M.; Lee, I. H., Biological activities of synthetic analogs of halocidin, an antimicrobial peptide from the tunicate Halocynthia aurantium. Antimicrobial agents and chemotherapy 2003, 47 (8), 2481-2486.

62. Suresh, A.; Verma, C., Modelling study of dimerization in mammalian defensins. $B M C$ bioinformatics 2006, 7 (5), S17.

63. Robey, F. A.; Fields, R. L., Automated synthesis of N-bromoacetyl-modified peptides for the preparation of synthetic peptide polymers, peptide-protein conjugates, and cyclic peptides. Analytical biochemistry 1989, 177 (2), 373-377.

64. Hall, K.; Mozsolits, H.; Aguilar, M.-I., Surface plasmon resonance analysis of antimicrobial peptide-membrane interactions: affinity \& mechanism of action. Letters in Peptide Science 2003, 10 (5-6), 475-485.

65. Steinberg, D. A.; Lehrer, R. I., Designer assays for antimicrobial peptides. Disputing the "onesize-fits-all" theory. Methods Mol Biol 1997, 78, 169-86.

66. Yeaman, M. R.; Yount, N. Y., Mechanisms of antimicrobial peptide action and resistance. Pharmacological reviews 2003, 55 (1), 27-55. 
67. Oren, Z.; Shai, Y., Mode of action of linear amphipathic $\alpha$ - helical antimicrobial peptides. Peptide Science 1998, 47 (6), 451-463.

68. Chen, Y.; Mant, C. T.; Farmer, S. W.; Hancock, R. E.; Vasil, M. L.; Hodges, R. S., Rational design of $\alpha$-helical antimicrobial peptides with enhanced activities and specificity/therapeutic index. Journal of Biological Chemistry 2005, 280 (13), 12316-12329.

69. Behrendt, R.; White, P.; Offer, J., Advances in Fmoc solid - phase peptide synthesis. Journal of Peptide Science 2016, 22 (1), 4-27.

70. Sarin, V. K.; Kent, S. B.; Tam, J. P.; Merrifield, R. B., Quantitative monitoring of solid-phase peptide synthesis by the ninhydrin reaction. Analytical biochemistry 1981, 117 (1), 147-157. 71.Van Bambeke, F.; Van Laethem, Y.; Courvalin, P.; Tulkens, P. M., Glycopeptide antibiotics. Drugs 2004, 64 (9), 913-936.

72. Dias, D. A.; Urban, S.; Roessner, U., A historical overview of natural products in drug discovery. Metabolites 2012, 2 (2), 303-336.

73. Hiramatsu, K., Vancomycin resistance in staphylococci. Drug resistance updates 1998, 1 (2), 135-150.

74. Biavasco, F.; Giovanetti, E.; Miele, A.; Vignaroli, C.; Facinelli, B.; Varaldo, P., In vitro conjugative transfer of VanA vancomycin resistance betweenEnterococci andListeriae of different species. European Journal of Clinical Microbiology and Infectious Diseases 1996, 15 (1), 50-59.

75. Uttley, A. C.; Collins, C.; Naidoo, J.; George, R., Vancomycin-resistant enterococci. The Lancet 1988, 331 (8575), 57-58.

76. Yarlagadda, V.; Akkapeddi, P.; Manjunath, G. B.; Haldar, J., Membrane active vancomycin analogues: a strategy to combat bacterial resistance. Journal of medicinal chemistry 2014, 57 (11), 4558-4568.

77. Okano, A.; Nakayama, A.; Schammel, A. W.; Boger, D. L., Total Synthesis of [ $\Psi$ [C $(\cdot \mathrm{NH}) \mathrm{NH}]$ Tpg4] Vancomycin and its (4-Chlorobiphenyl) methyl Derivative: Impact of Peripheral Modifications on Vancomycin Analogues Redesigned for Dual d-Ala-d-Ala and d-Ala-d-Lac Binding. Journal of the American Chemical Society 2014, 136 (39), 13522-13525.

78. Ge, M.; Chen, Z.; Russell, H.; Kohler, J.; Silver, L. L.; Kerns, R.; Fukuzawa, S.; Thompson, C.; Kahne, D., Vancomycin derivatives that inhibit peptidoglycan biosynthesis without binding DAla-D-Ala. Science 1999, 284 (5413), 507-511.

79. Nagarajan, R.; Schabel, A.; Occolowitz, J.; Counter, F.; Ott, J.; Felty-Duckworth, A., Synthesis and antibacterial evaluation of $\mathrm{N}$-alkyl vancomycins. The Journal of antibiotics 1989, 42 (1), 63-72.

80. Cooper, R. D.; Snyder, N. J.; Zweifel, M. J.; Staszak, M. A.; Wilkie, S. C.; Nicas, T. I.; Mullen, D. L.; Butler, T. F.; Rodriguez, M. J.; Huff, B. E., Reductive alkylation of glycopeptide antibiotics: synthesis and antibacterial activity. The Journal of antibiotics 1996, 49 (6), 575-581.

81. Arimoto, H.; Nishimura, K.; Hayakawa, I.; Kinumi, T.; Uemura, D., Multi-valent polymer of vancomycin: enhanced antibacterial activity against VRE. Chemical communications 1999, (15), 1361-1362.

82. Mu, Y.; Nodwell, M.; Pace, J. L.; Shaw, J.-P.; Judice, J. K., Vancomycin disulfide derivatives as antibacterial agents. Bioorganic \& medicinal chemistry letters 2004, 14 (3), 735-738.

83. Yoshida, O.; Yasukata, T.; Sumino, Y.; Munekage, T.; Narukawa, Y.; Nishitani, Y., Novel semisynthetic glycopeptide antibiotics active against methicillin-resistant staphylococcus aureus (MRSA) and vancomycin-resistant Enterococci (VRE): doubly-modified water-soluble 
derivatives of chloroorienticin B. Bioorganic \& medicinal chemistry letters 2002, 12 (21), 3027-3031.

84. Pavlov, A.; Berdnikova, T.; Olsufyeva, E.; Lazhko, E.; Malkova, I.; Preobrazhenskaya, M.; Testa, R.; Petersen, P., Synthesis and biological activity of derivatives of glycopeptide antibiotics eremomycin and vancomycin nitrosated, acylated or carbamoylated at the $\mathrm{N}$-terminal. The Journal of antibiotics 1993, 46 (11), 1731-1739.

85. Shi, Z.; Griffin, J. H., Catalysis of carbamate hydrolysis by vancomycin and semisynthetic derivatives. Journal of the American Chemical Society 1993, 115 (15), 6482-6486.

86. Crowley, B. M.; Boger, D. L., Total synthesis and evaluation of [ $\Psi[\mathrm{CH} 2 \mathrm{NH}] \mathrm{Tpg} 4]$ vancomycin aglycon: reengineering vancomycin for dual D-Ala-D-Ala and D-Ala-D-Lac binding. Journal of the American Chemical Society 2006, 128 (9), 2885-2892.

87. Malabarba, A.; Trani, A.; Strazzolini, P.; Cietto, G.; Ferrari, P.; Tarzia, G.; Pallanza, R.; Berti, M., Synthesis and biological properties of N63-carboxamides of teicoplanin antibiotics. Structureactivity relationships. Journal of medicinal chemistry 1989, 32 (11), 2450-2460.

88. Malabarba, A.; Nicas, T. I.; Thompson, R. C., Structural modifications of glycopeptide antibiotics. Medicinal research reviews 1997, 17 (1), 69-137.

89. Beauregard, D. A.; Williams, D. H.; Gwynn, M. N.; Knowles, D., Dimerization and membrane anchors in extracellular targeting of vancomycin group antibiotics. Antimicrobial agents and chemotherapy 1995, 39 (3), 781-785.

90. Han, J. C.; Han, G. Y., A procedure for quantitative determination of tris (2-carboxyethyl) phosphine, an odorless reducing agent more stable and effective than dithiothreitol. Analytical biochemistry 1994, 220 (1), 5-10.

91. Tam, J. P.; Wu, C.; Yang, J. L., Membranolytic selectivity of cystine - stabilized cyclic protegrins. European journal of biochemistry 2000, 267 (11), 3289-3300.

92. T. Kaeberlein; K. Lewis*; Epstein, S. S., Isolating "Uncultivable" Microorganisms in Pure Culture in a Simulated Natural Environment. Science 2002, Vol. 296, Issue 5570, pp. $1127-$ 1129 (Issue 5570), pp. 1127-1129

93. Sherpa, R. T.; Reese, C. J.; Montazeri Aliabadi, H., Application of iChip to Grow "Uncultivable" Microorganisms and its Impact on Antibiotic Discovery. Journal of pharmacy \& pharmaceutical sciences : a publication of the Canadian Society for Pharmaceutical Sciences, Societe canadienne des sciences pharmaceutiques 2015, 18 (3), 303-15.

94. Hunter, P., Antibiotic discovery goes underground: The discovery of teixobactin could revitalise the search for new antibiotics based on the novel method the researchers used to identify the compound. EMBO reports 2015, 16 (5), 563-5.

95. Nichols, D.; Cahoon, N.; Trakhtenberg, E. M.; Pham, L.; Mehta, A.; Belanger, A.; Kanigan, T.; Lewis, K.; Epstein, S. S., Use of ichip for high-throughput in situ cultivation of "uncultivable" microbial species. Applied and environmental microbiology 2010, 76 (8), 2445-50.

96. von Nussbaum, F.; Sussmuth, R. D., Multiple attack on bacteria by the new antibiotic teixobactin. Angewandte Chemie 2015, 54 (23), 6684-6.

97. Giltrap, A. M.; Dowman, L. J.; Nagalingam, G.; Ochoa, J. L.; Linington, R. G.; Britton, W. J.; Payne, R. J., Total Synthesis of Teixobactin. Organic letters 2016, 18 (11), 2788-91.

98. Sivanathan, S.; Scherkenbeck, J., Cyclodepsipeptides: a rich source of biologically active compounds for drug research. Molecules (Basel, Switzerland) 2014, 19 (8), 12368-420. 
99. Lopez-Macia, A.; Jimenez, J. C.; Royo, M.; Giralt, E.; Albericio, F., Synthesis and structure determination of kahalalide F (1,2). J Am Chem Soc 2001, 123 (46), 11398-401.

100. Robbel, L.; Marahiel, M. A., Daptomycin, a bacterial lipopeptide synthesized by a nonribosomal machinery. The Journal of biological chemistry 2010, 285 (36), 27501-8.

101. Arbeit, R. D.; Maki, D.; Tally, F. P.; Campanaro, E.; Eisenstein, B. I., The safety and efficacy of daptomycin for the treatment of complicated skin and skin-structure infections. Clinical infectious diseases : an official publication of the Infectious Diseases Society of America 2004, 38 (12), 1673-81.

102. Sakoulas, G.; Moise-Broder, P. A.; Schentag, J.; Forrest, A.; Moellering, R. C., Jr.; Eliopoulos, G. M., Relationship of MIC and bactericidal activity to efficacy of vancomycin for treatment of methicillin-resistant Staphylococcus aureus bacteremia. Journal of clinical microbiology 2004, 42 (6), 2398-402.

103. Johnson, A. P.; Uttley, A.; Woodford, N.; George, R., Resistance to vancomycin and teicoplanin: an emerging clinical problem. Clinical Microbiology Reviews 1990, 3 (3), 280-291.

104. Parmar, A.; lyer, A.; Vincent, C. S.; Van Lysebetten, D.; Prior, S. H.; Madder, A.; Taylor, E. J.; Singh, I., Efficient total syntheses and biological activities of two teixobactin analogues. Chemical communications 2016, 52 (36), 6060-3.

105. Yang, H.; Chen, K. H.; Nowick, J. S., Elucidation of the Teixobactin Pharmacophore. ACS chemical biology 2016.

106. SCHMIDT, U.; LANGNER, J., Cyclotetrapeptides and cyclopentapeptides: occurrence and synthesis. The Journal of peptide research 1997, 49 (1), 67-73.

107. Hughes, A. B., Amino Acids, Peptides and Proteins in Organic Chemistry, Analysis and Function of Amino Acids and Peptides. John Wiley \& Sons: 2013; Vol. 5.

108. Rohrbacher, F.; Deniau, G.; Luther, A.; Bode, J. W., Spontaneous head-to-tail cyclization of unprotected linear peptides with the KAHA ligation. Chemical Science 2015, 6 (8), 4889-4896.

109. Camarero, J. A.; Cairó, J. J.; Giralt, E.; Andreu, D., Solution versus solid - phase cyclization strategies for large sidechain lactam - bridged peptides: A comparative study. Journal of Peptide Science 1995, 1 (4), 241-250.

110. Cabrele, C.; Langer, M.; Beck-Sickinger, A. G., Amino acid side chain attachment approach and its application to the synthesis of tyrosine-containing cyclic peptides. The Journal of Organic Chemistry 1999, 64 (12), 4353-4361.

111. Jensen, K. J.; Alsina, J.; Songster, M. F.; Vágner, J.; Albericio, F.; Barany, G., Backbone Amide Linker (BAL) Strategy for Solid-Phase Synthesis of C-Terminal-Modified and Cyclic Peptides1, 2, 3. Journal of the American Chemical Society 1998, 120 (22), 5441-5452.

112. Humphrey, J. M.; Chamberlin, A. R., Chemical synthesis of natural product peptides: coupling methods for the incorporation of noncoded amino acids into peptides. Chemical Reviews 1997, 97 (6), 2243-2266.

113. Yan, L. Z.; Dawson, P. E., Synthesis of peptides and proteins without cysteine residues by native chemical ligation combined with desulfurization. Journal of the American Chemical Society 2001, 123 (4), 526-533.

114. Perlstein, M. T.; Atassi, M. Z.; Cheng, S. H., Desulfurization of sulfur amino acids and proteins with Raney nickel. Biochimica Et Biophysica Acta 1971, 236 (1), 174-182. 


\section{APPENDIX}

The NMR spectra of the compounds are attached in this section. Vancomycin carboxamide analogs were prepared as mentioned in Chapter 3. In additional to ESI-MS of compounds mentioned in the section 3.2.2. The NMR spectra of four analogs are shown here. ${ }^{1} \mathrm{H}$ NMR experiments were conducted using a Bruker $400 \mathrm{MHz}$ spectrometer at $25^{\circ} \mathrm{C}$.

\section{NMR spectra of Chapter 3 compounds}

\section{IH NMR spectrum of Van-diaminopropane (Chapter 3, compound 7)}

\section{(DMSO-d $6,400 \mathrm{MHz}):$}

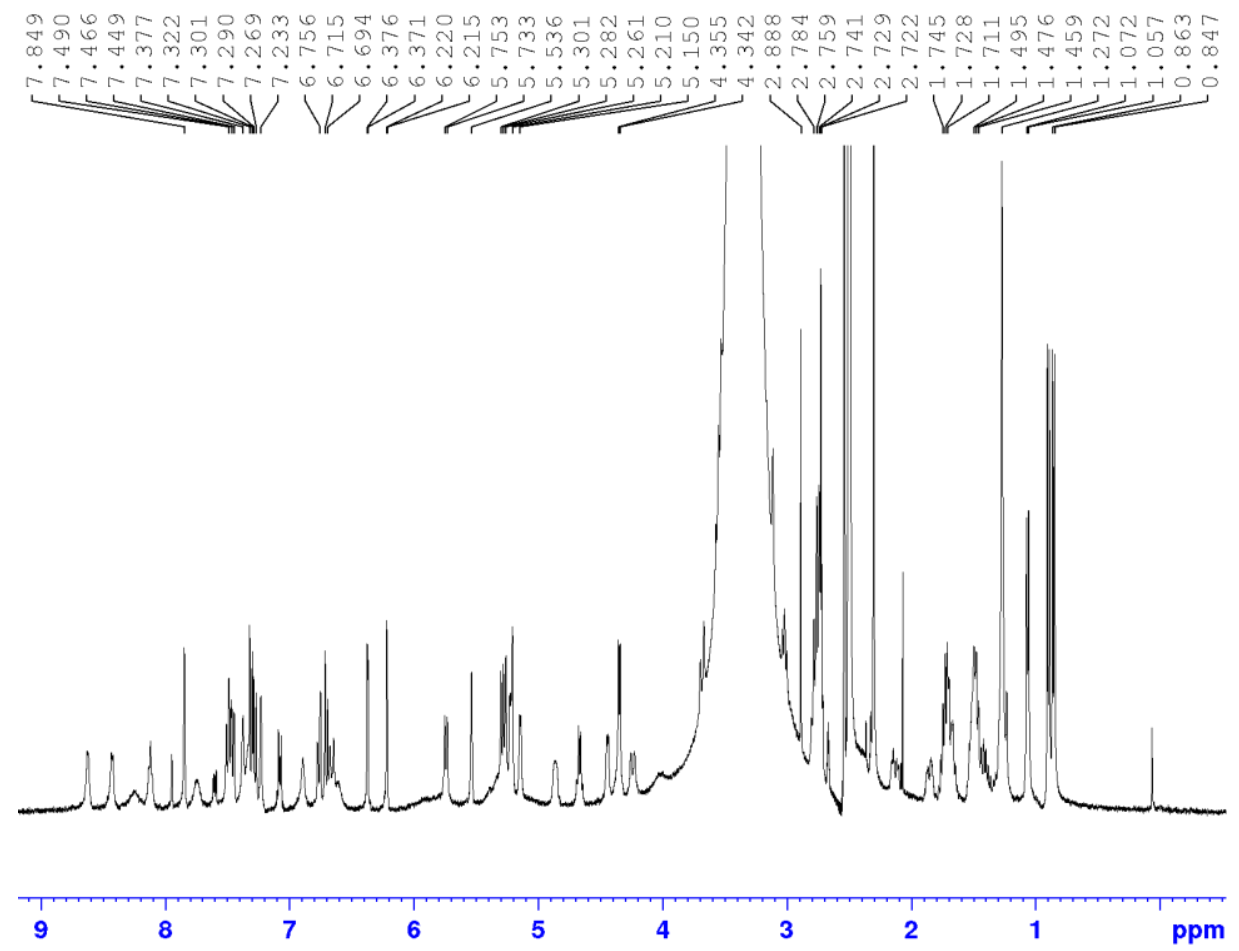




\section{IH NMR spectrum of Van-diaminopentane(Chapter 3, compound 9)}

(DMSO-d6, $400 \mathrm{MHz}):$

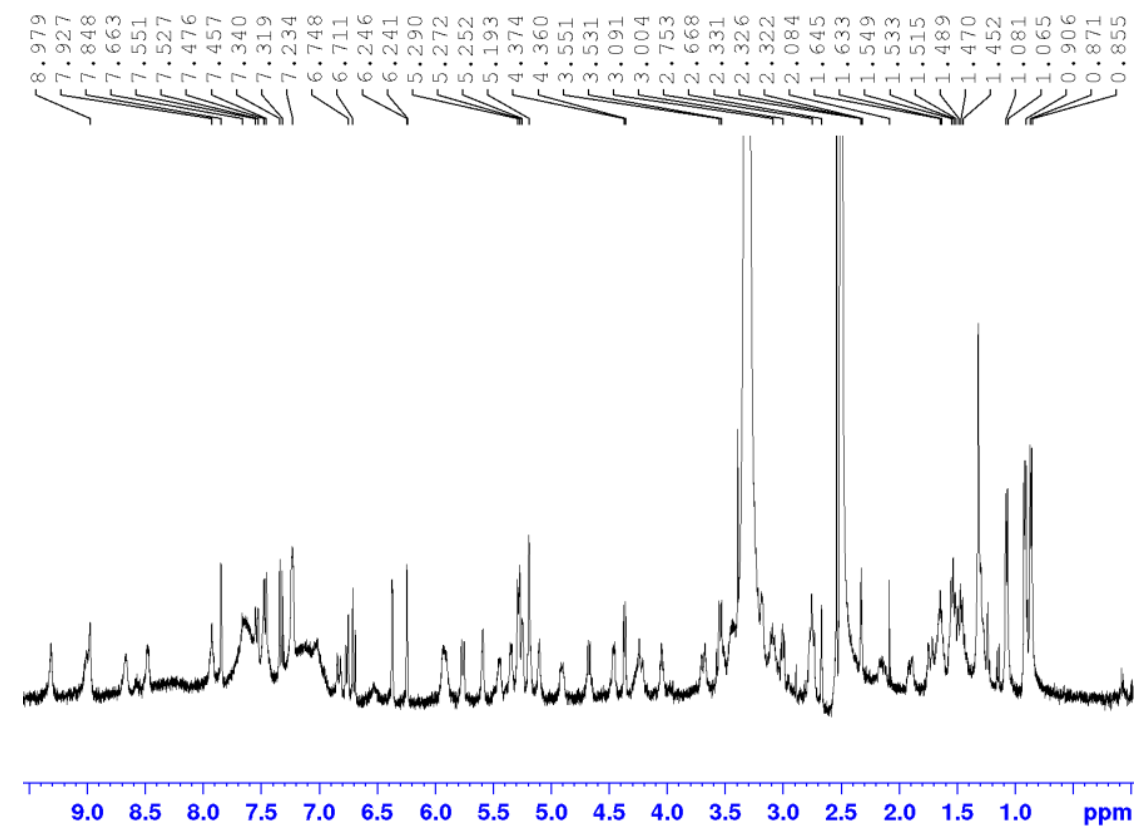

IH NMR spectrum of Van-diaminohexane(Chapter 3, compound 10) (DMSOd6, $400 \mathrm{MHz}):$

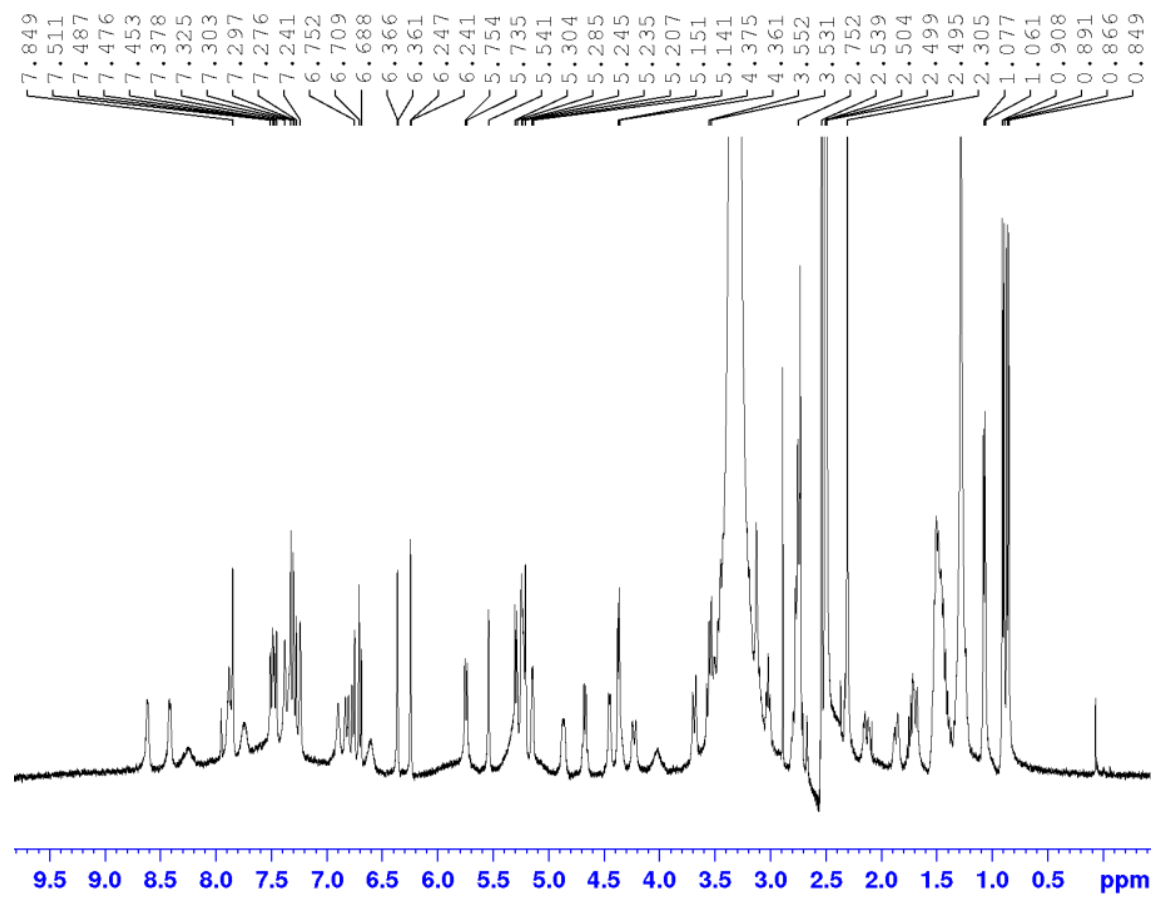


IH NMR spectrum of Van-O-phenylenediamine(Chapter 3, compound 12) (DMSO-d $6,400 \mathrm{MHz}):$

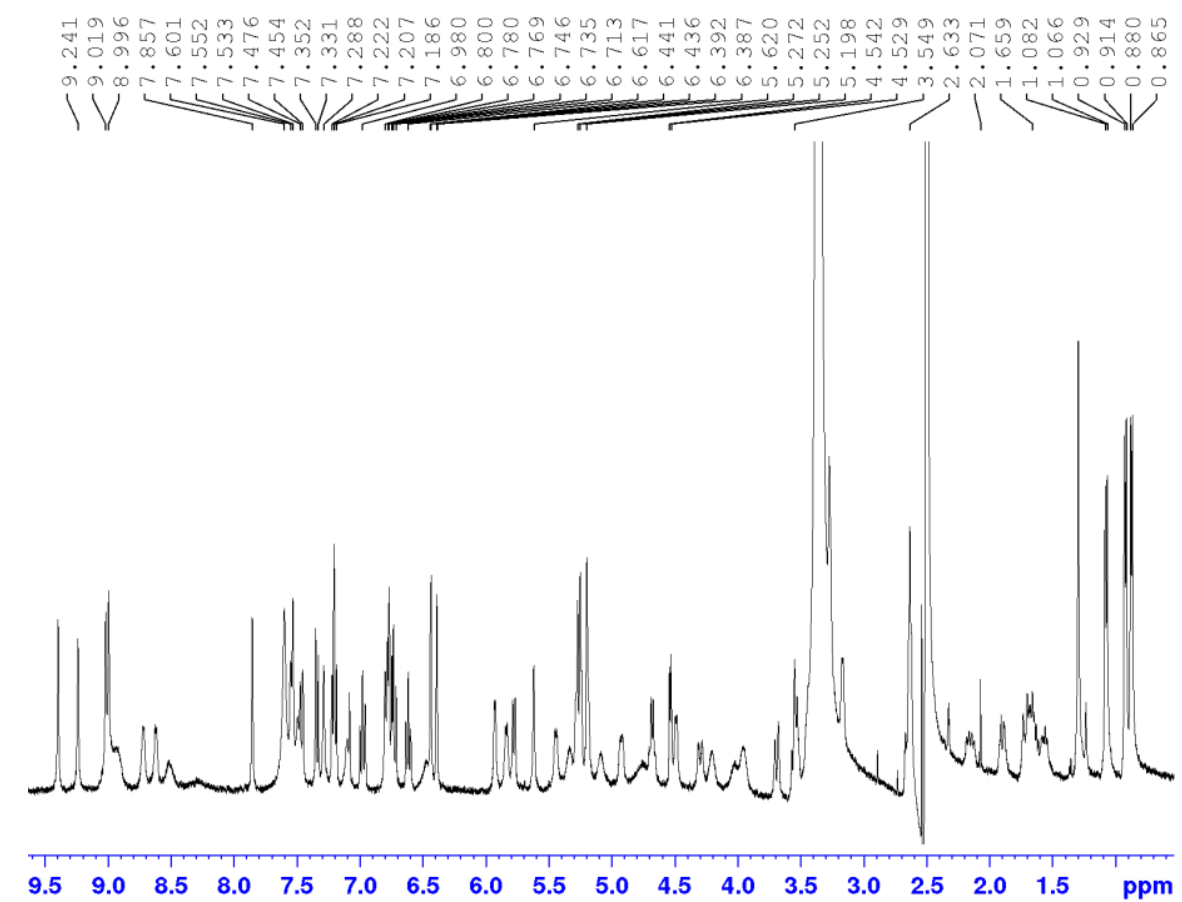

IH NMR spectrum of azido-isoleucine (Chapter 4) (DMSO-d $6,400 \mathrm{MHz}$ ):

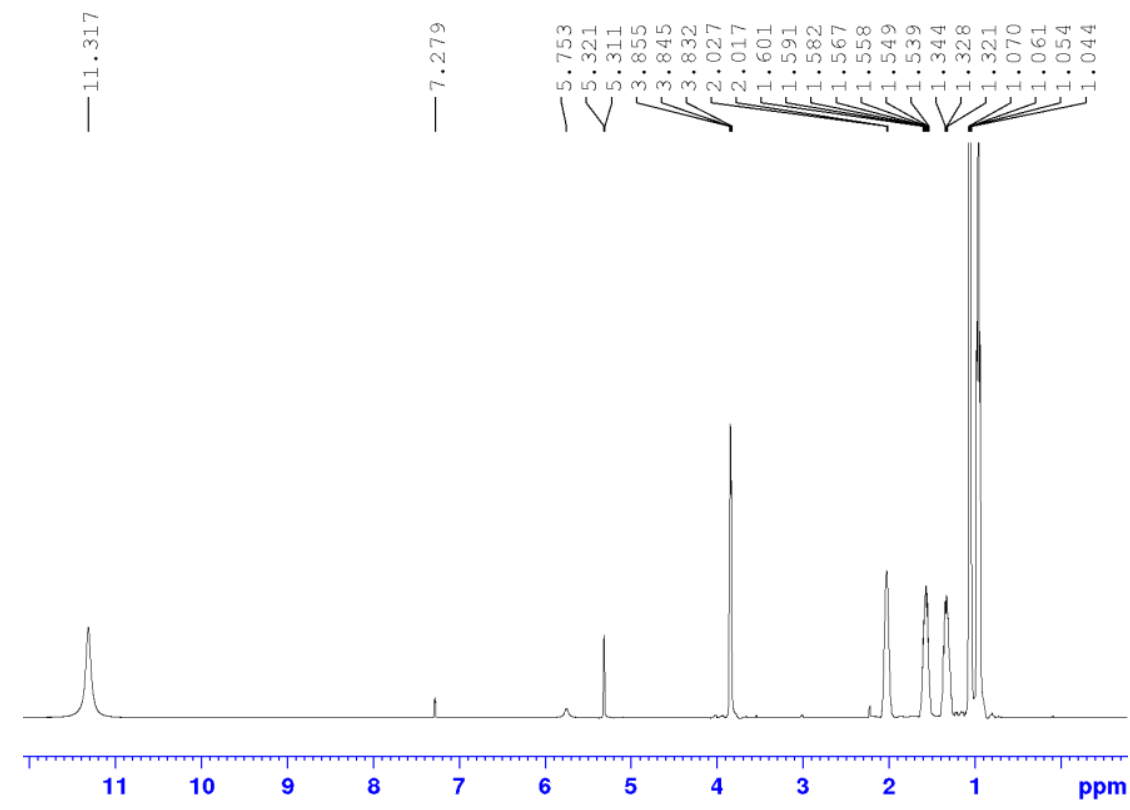




\section{NMR spectra and HRMS of Chapter-4 compounds}

The NMR experiments of four teixobactin analogs (in DMSO-D6) were conducted in Bruker Avance II $700-\mathrm{MHz}$ spectrometer at $25^{\circ} \mathrm{C}$. In previous publications that reported Arg10-teixbactin, Lys10-teixobactin, Orn10-teixobactin, the $\mathrm{pH}$ conditions were not mentioned. We employed $\mathrm{pH} 5$ in our experiments.

IH NMR spectrum of Arg10-teixobactin (Chapter 4, compound 11a) in DMSO-d6

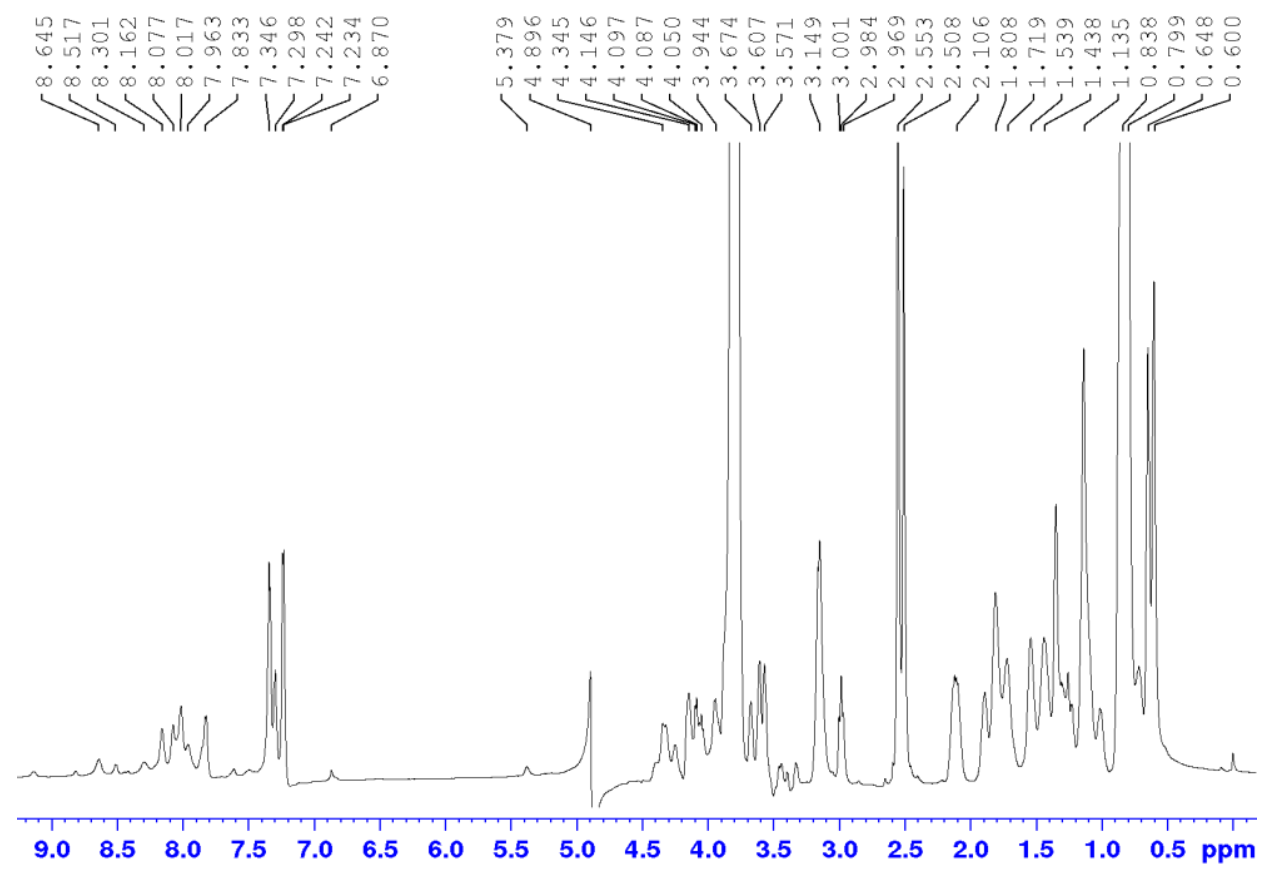

HRMS data of Arg10-teixobactin

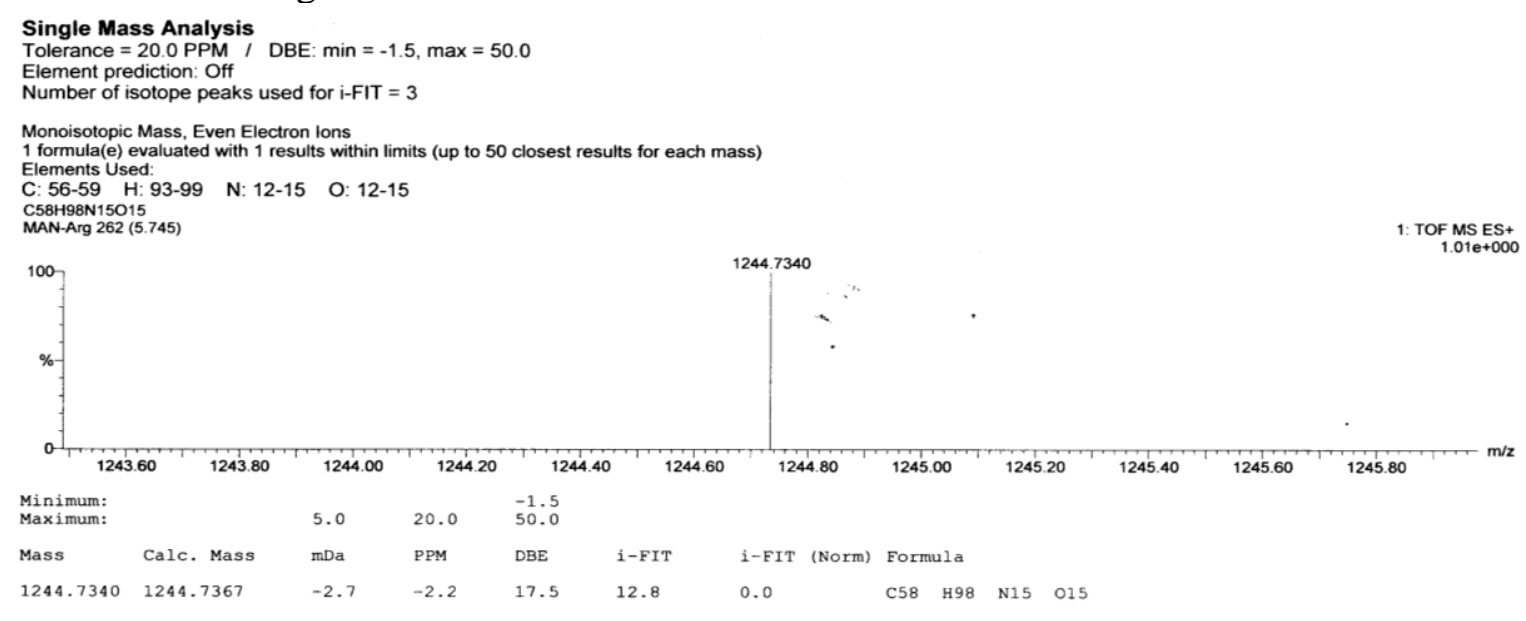




\section{IH NMR spectrum of Lys10-teixobactin (Chapter 4, compound 12a) in DMSO-}

$\underline{\mathbf{d}_{6}}$

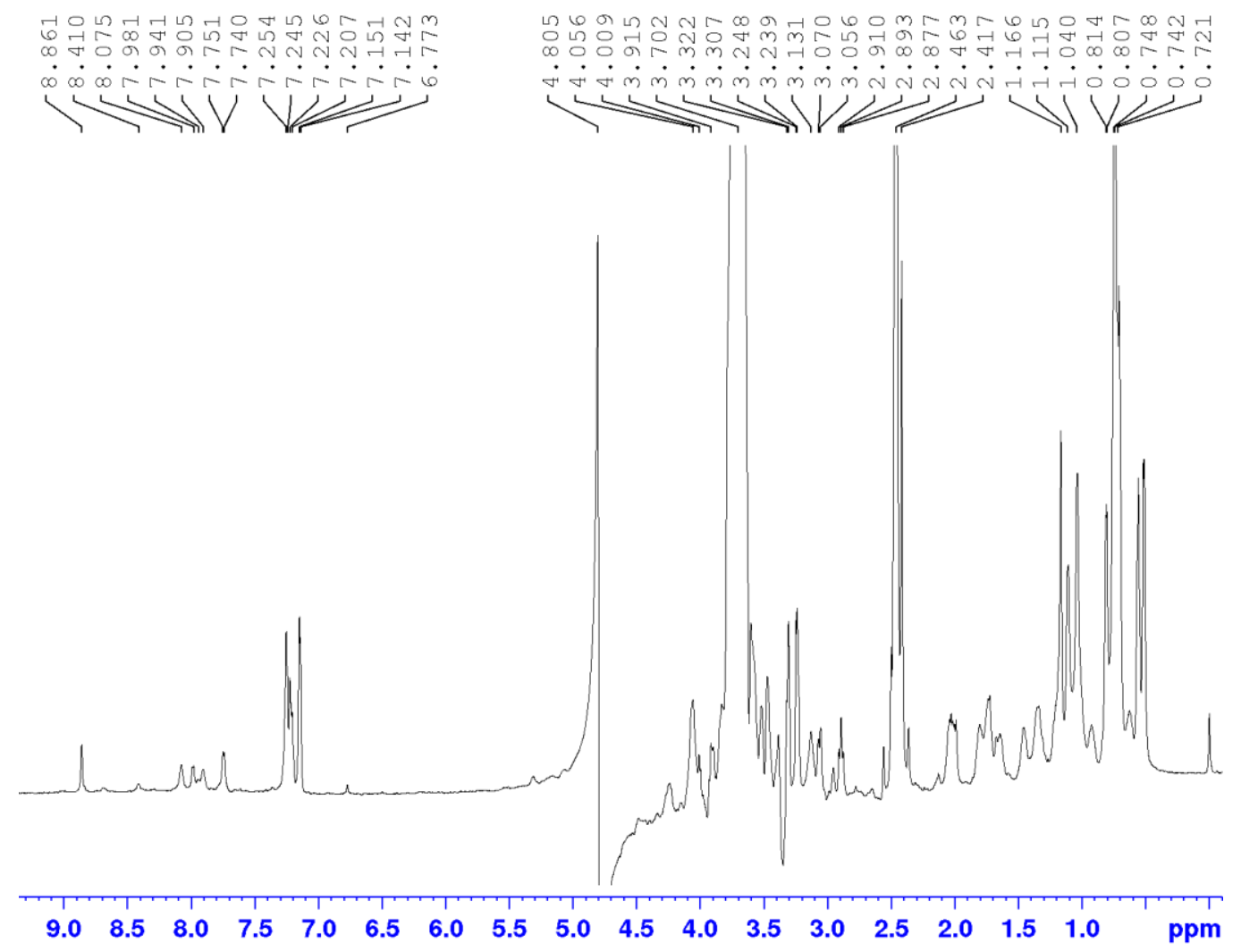

HRMS data of Lys10-teixobactin

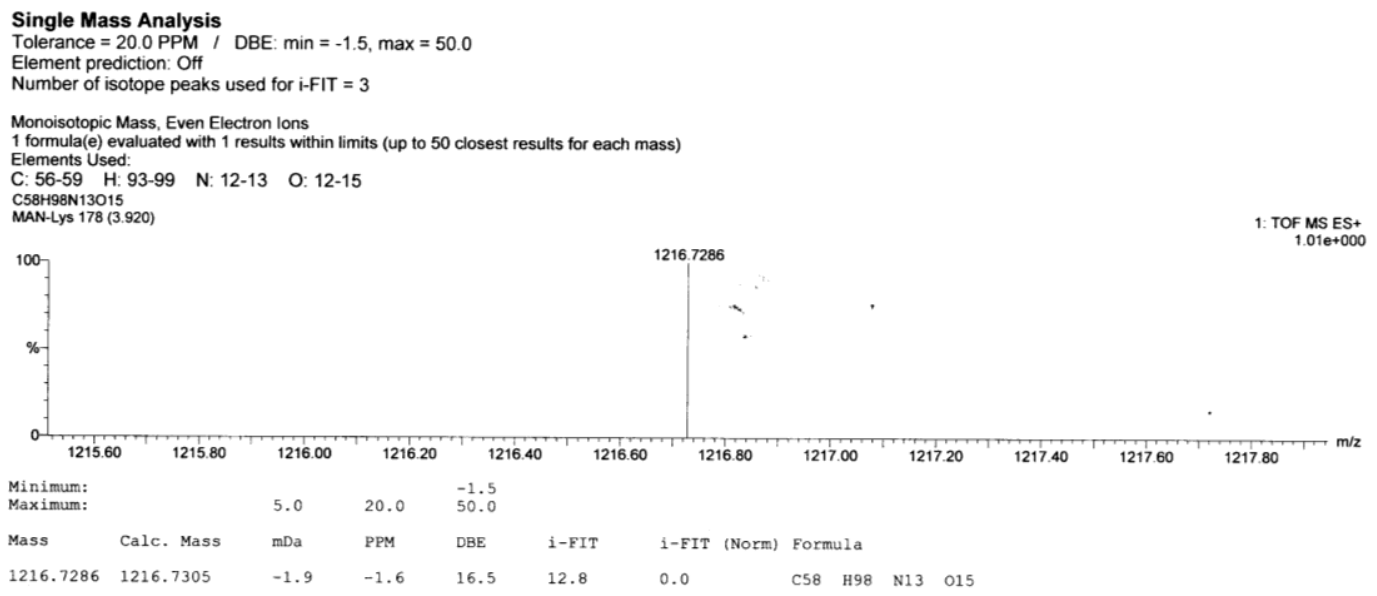




\section{IH NMR spectrum of Orn10-teixobactin (Chapter 4, compound 13a) in DMSO-}

$\mathbf{d}_{6}$

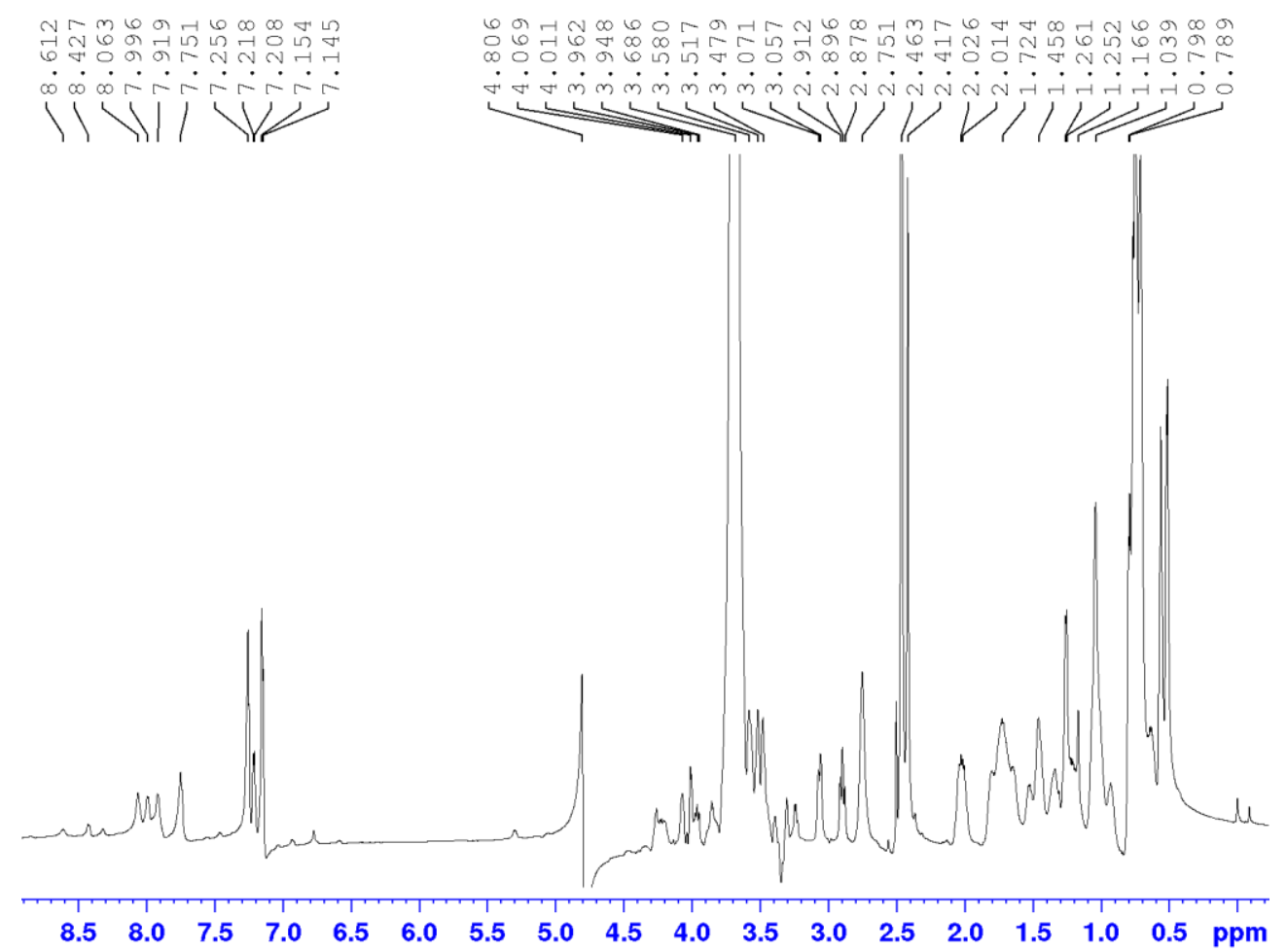

HRMS data of Orn10-teixobactin

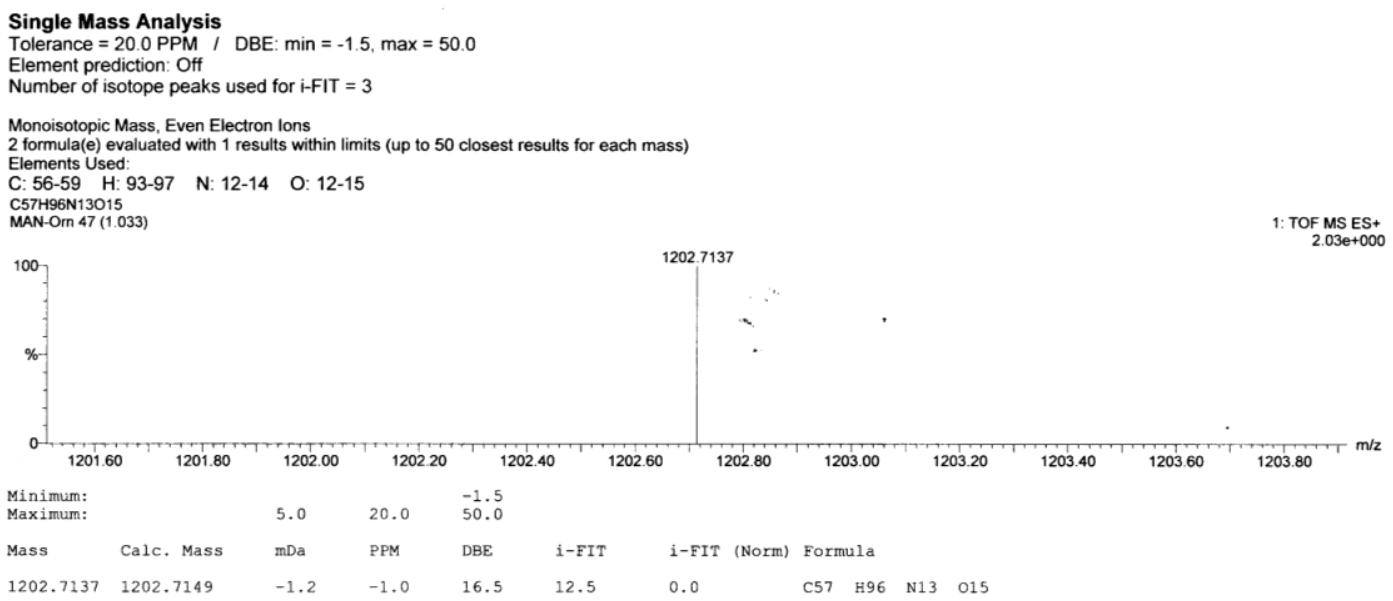


IH NMR spectrum of His10-teixobactin (Chapter 4, compound 14a) in DMSO-d6

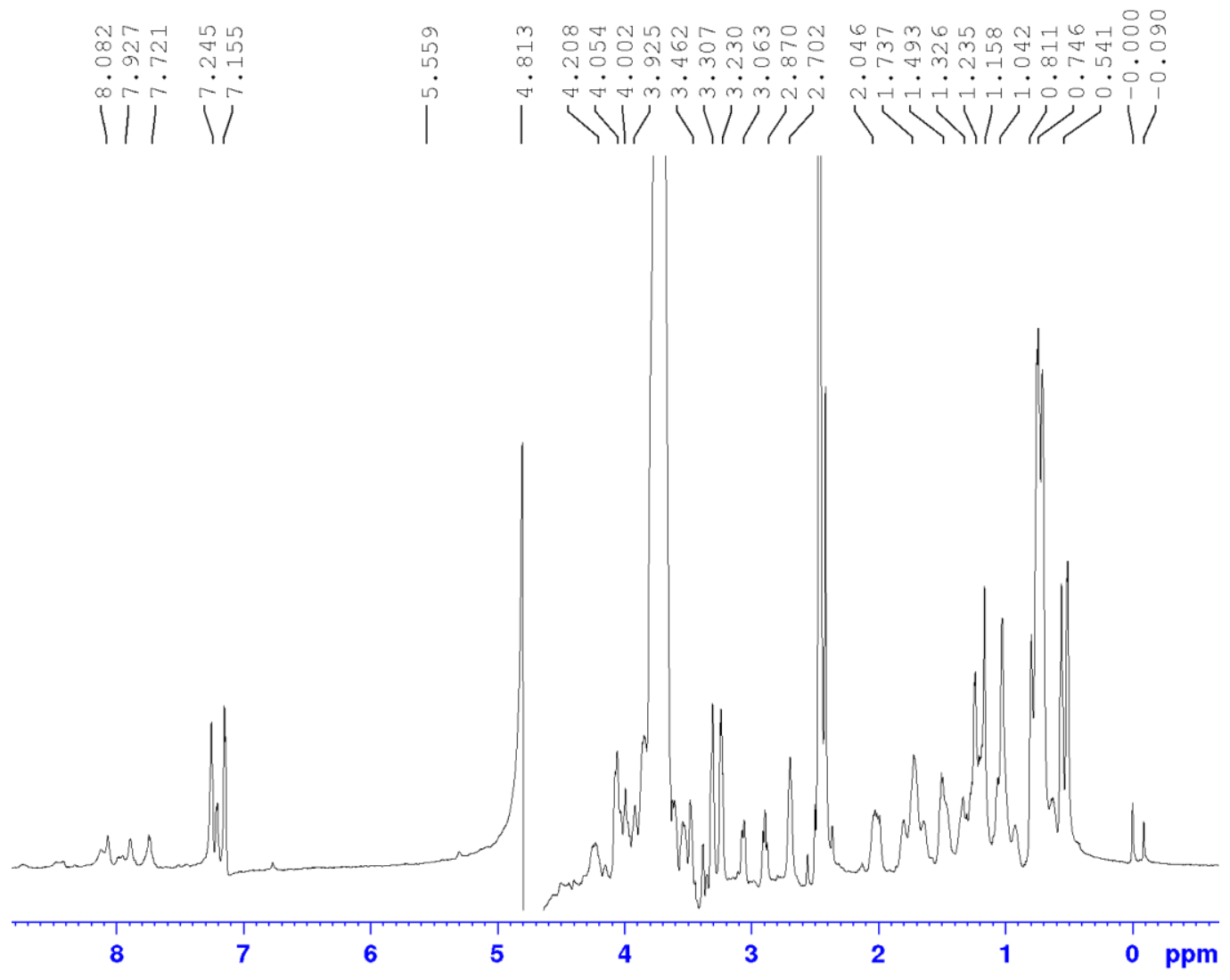

HRMS data of His10-teixobactin 
Single Mass Analysis

Tolerance $=20.0$ PPM DBE: $\min =-1.5, \max =50.0$

Element prediction Off

Number of isotope peaks used for $\mathrm{i}-\mathrm{FIT}=3$

Monoisotopic Mass, Even Electron lons

5 formula(e) evaluated with 1 results within limits (up to 50 closest results for each mass)

Elements Used:

$\begin{array}{llll}\text { C: } 56-59 & \text { H: } 93-99 & \mathrm{~N}: 12-15 & \text { O: } 12-15\end{array}$

C58H93N14O15

MAN-His 191 (4.189)

1: TOF MS ES+

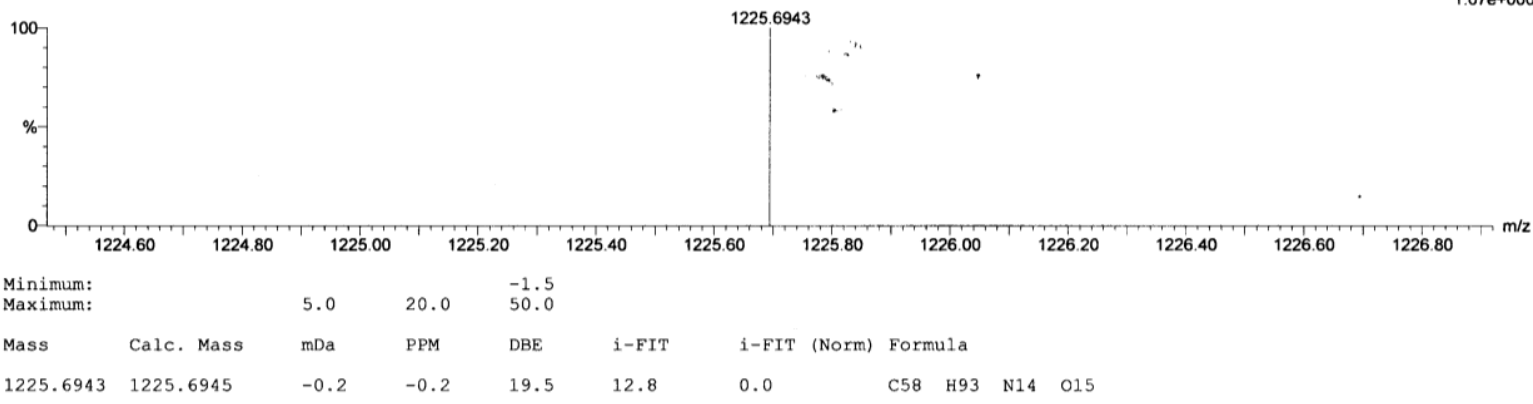

